DESIGN AND DEVELOPMENT OF A TORNADO

INTERCEPT UNMANNED AERIAL VEHICLE

\author{
By \\ ANDREW LEVI ROSS \\ Bachelor of Science in Mechanical and Aerospace \\ Engineering \\ Oklahoma State University \\ Stillwater, Oklahoma \\ 2017
}
Submitted to the Faculty of the
Graduate College of the
Oklahoma State University
in partial fulfillment of the requirements for the Degree of
MASTER OF SCIENCE

December, 2019 


\section{DESIGN AND DEVELOPMENT OF A TORNADO INTERCEPT UNMANNED AERIAL VEHICLE}

Thesis Approved:

Dr. Jamey Jacob

Thesis Adviser

Dr. Andrew Arena

Dr. Imraan Faruque 


\section{ACKNOWLEDGEMENTS}

I would like to acknowledge those who aided in the development of the aircraft, including but not limited: the entire Speedfest 2017 Orange Team for aiding in the initial design, building, and flight testing of the original aircrafts; Marc Hartman for being an exceptional pilot even when the aircraft experienced several instances of mid-flight engine failure; Colton Dancer and Charlie Johnson not only for helping in the layup process of the skins, but also diving into the Pixhawk parameter files to make necessary changes early in the development process (Colton) and continuing that work through the changing versions (Charlie); Josh Hartman for doing the initial wiring of the prototype aircraft; Kylar Murray for being the first to successfully create an integrated winglet hatch; Jeff Sandwell for helping debug turbine issues as they arose; Seabrook Whyte and Ben Loh for additional insight on the launcher build process, and many others around Oklahoma State's MAE department.

I would also like to thank Dr. Jamey Jacob for providing the opportunity to make this project a reality, Dr. Andrew Arena for creating Speedfest (which was the foundation for this entire project), and Dr. Imraan Faruque for all his insight on controls and the aerodynamic modeling process. This project would not have been possible without their guidance and patience.

This work is supported in part by the National Science Foundation under Grant No. 1539070, Collaboration Leading Operational UAS Development for Meteorology and Atmospheric Physics (CLOUD-MAP), to Oklahoma State University.

Acknowledgements reflect the views of the author and are not endorsed by committee members or Oklahoma State University. 


\section{Name: ANDREW LEVI ROSS}

Date of Degree: DECEMBER, 2019

\section{Title of Study: DESIGN AND DEVELOPMENT OF A TORNADO INTERCEPT UNMANNED AERIAL VEHICLE}

\section{Major Field: MECHANICAL AND AEROSPACE}

Abstract: Very little data surrounding tornadogenesis exists, and this is due in part to the lack of tools necessary to deliver weather-sensing instruments into the severe weather phenomenon. Though current UAS are able to accurately gather weather data, they typically cannot operate in high winds or in rain. To help bridge this data gap, a custom delta wing UAS was developed to withstand both of these shortcomings.

The aircraft was outfitted with an autopilot, long range RC control, and FPV systems for control over long distances. Despite utilizing a new turbojet that generates $50 \%$ more thrust at the cost of a 50\% increase to fuel consumption, custom fuel tanks were designed and fabricated double the flight time of the previous system. Considerations for preventing ingested water from damaging internal avionics are explored, as operation in rain is a requirement.

Aircraft performance approximations were generated using both analytical approximations and flight test data from the autopilot's flight log. An approach to generating a longitudinal aerodynamic control model is explored to estimate performance in extreme winds $(75+\mathrm{mph})$, but ultimately fails due to lack of conventional horizontal tail and the coupling of pitch and yaw controls to the turbine's exhaust speed (controlled by throttle setting).

A car-based launcher system was also created for utilization in the field, reducing the required personnel's experience to execute a mission effectively. Multiple aircraft, as well as a prototype of the TIA, has launched successfully multiple times from this system, even exhibiting full auto-launching functionality.

The longest recorded flight was over a ground track of 22 miles with an average airspeed of 100 knots over 16 minutes, consuming $60 \%$ of the total fuel available. The top speed achieved by the aircraft was $160 \mathrm{mph}$ at $90 \%$ throttle. 
TABLE OF CONTENTS

$\begin{array}{ll}\text { Chapter } & \text { Page }\end{array}$

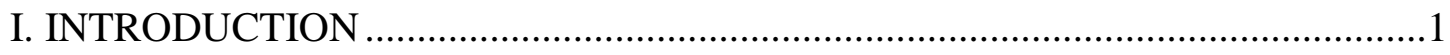

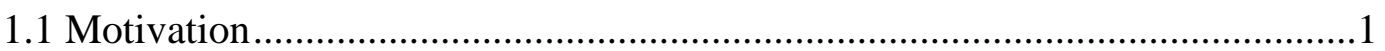



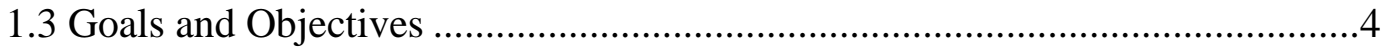

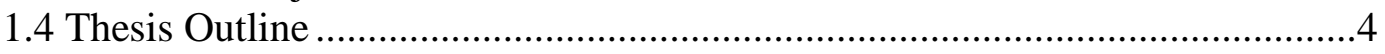

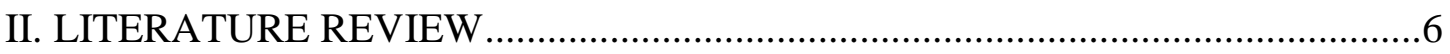

2.1 Current Atmospheric Sampling Methods ..........................................................6

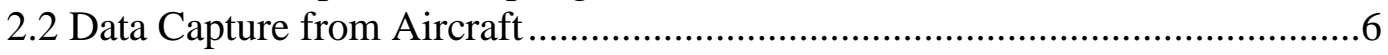

2.3 Takeaways for Aircraft Development...........................................................15

III. DESIGN REQUIREMENTS AND CONCEPTS ……………............................17

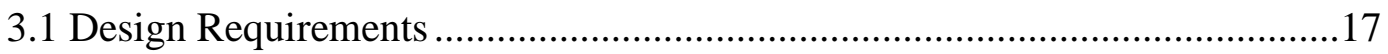

3.2 Aircraft Characteristic Down-selection ....................................................18

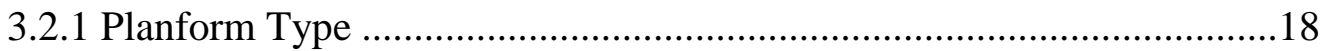

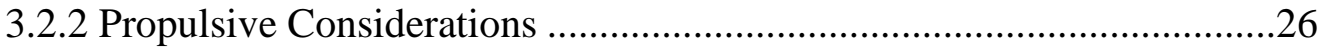

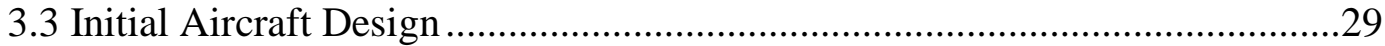

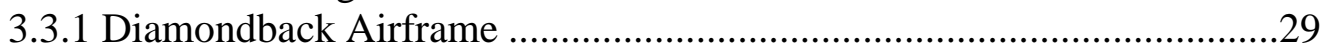

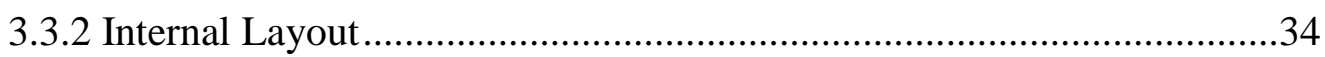

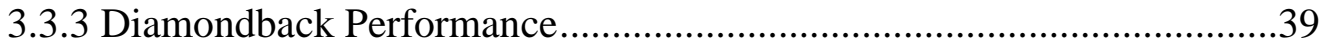

3.3.4 Comparison of Diamondback to Other Aircraft .......................................41

3.4 Structural/Internal Redesign .....................................................................42

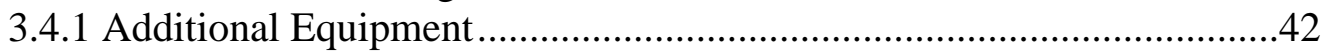

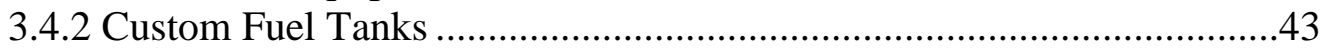

3.4.3 Long Range Autopilot Communications ...............................................44

3.4.4 FPV Video System...........................................................................46

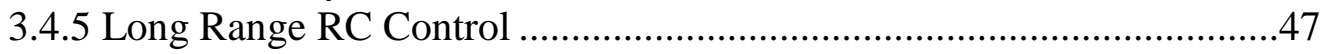

3.4.6 RF Energy Management ......................................................................48 


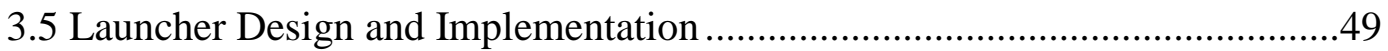

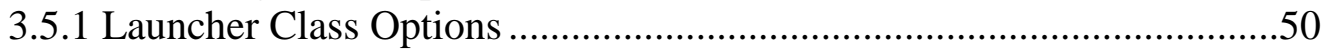

3.5.1.1 Hand Launch ..................................................................50

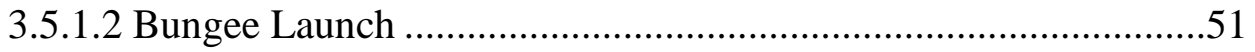

3.5.1.3 Catapult/Rail Launch ...........................................................52

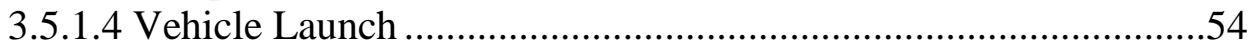

3.5.1.5 Chosen Class: Vehicle Launch .................................................57

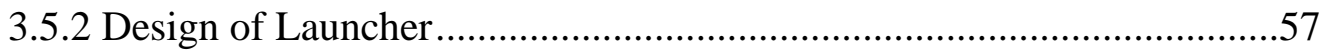

3.6 Controls Concepts and Methods ..........................................................61

3.6.1 General Controls Concept ...............................................................62

IV. SYSTEM INTEGRATION, BUILD OF AIRFRAME AND LAUNCHER .........66

4.1 Aircraft Flight System Integration .......................................................66

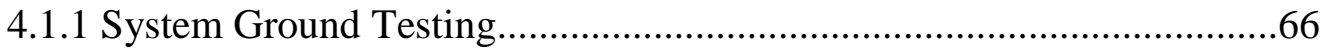

4.1.1.1 DragonLink RC ...........................................................67

4.1.1.2 Pixhawk Autopilot ........................................................68

4.1.1.3 FPV Video ....................................................................... 76

4.1.2 Flight Testing of Integrated Systems ............................................... 78

4.1.2.1 Intermittent Mid-Flight Turbine Shutdown ..............................78

4.1.2.2 Turbine Throttle Surging ...................................................80

4.1.2.3 Loss of Altitude During Bank ...............................................83

4.1.2.4 Launching Autonomously From Launcher ...............................83

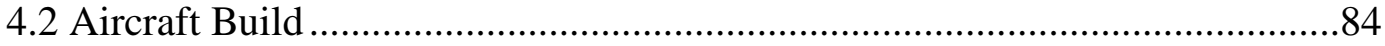

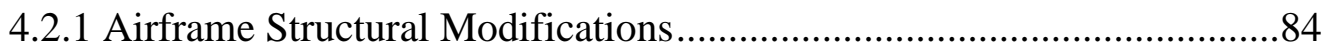

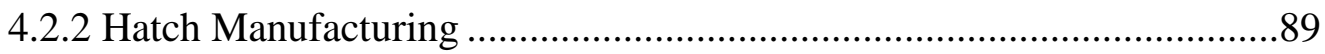

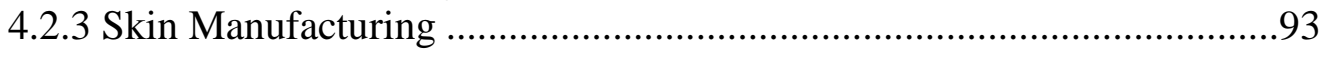

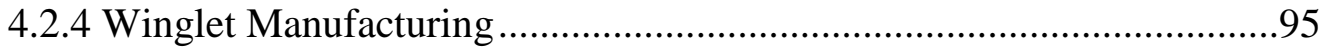

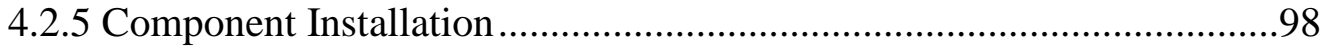

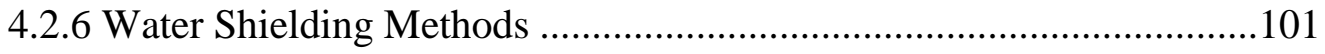

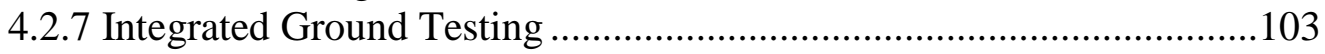

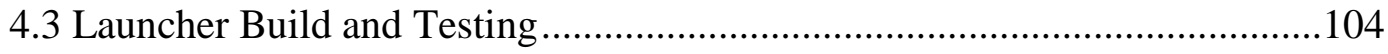

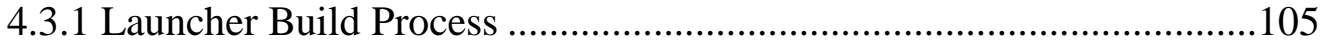

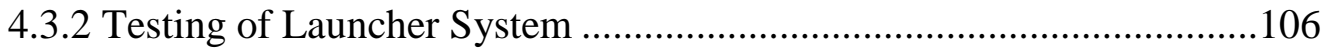

4.3.2.1 Testing Stability of System ................................................ 106

4.3.2.2 Flight Test Plan .................................................................107

4.3.2.3 Test 1: Firstar V2, Single Prop, Conventional Airframe ..........109

4.3.2.4 Test 2: Skyhunter, Single Prop, Conventional Airframe ..........111

4.3.2.5 Test 3: Believer, Dual Prop, Conventional Airframe ...............115

4.3.2.6 Test 4: Skywalker X8, Single Prop, Delta Wing .....................118

4.3.2.7 Test 5: Beast (Electric TIA Prototype), EDF, Delta wing ........123

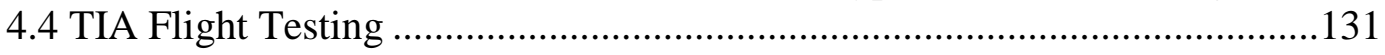

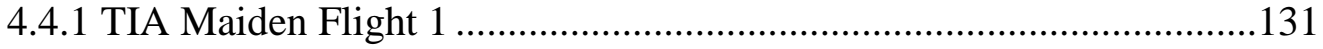

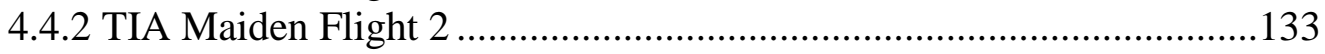




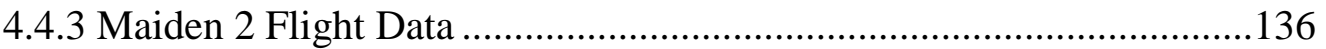

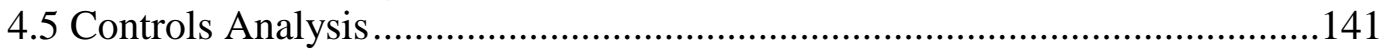

4.5.1 TIA Acquiring Coefficients ....................................................... 142

V. CONCLUSIONS: FLIGHT AND SYSTEM REVIEW OF AIRCRAFT ...........147

5.1 TIA Compared to Other Severe Weather UAS .......................................... 147

5.2 Summary of Aircraft Capabilities ..............................................................149

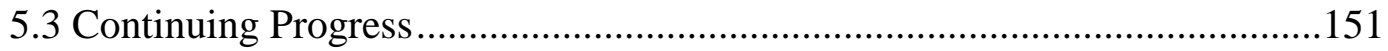

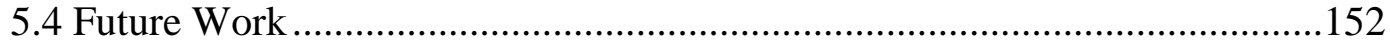

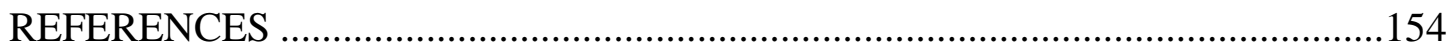

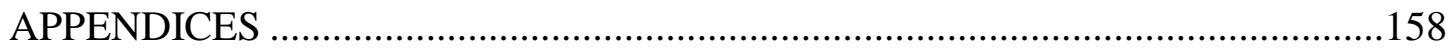

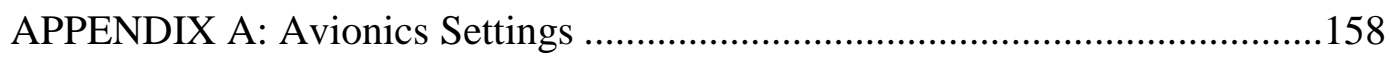

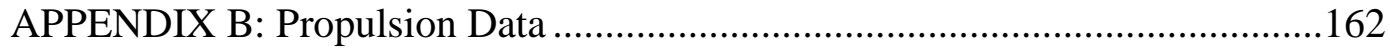



APPENDIX D: Launcher Wind Load Estimate..............................................173

APPENDIX E: Attempted Airfoils for XFOIL Analysis .................................174

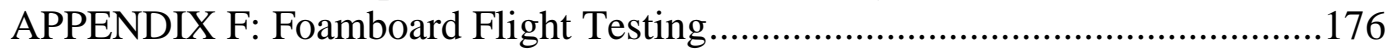

APPENDIX G: Matlab Flight Data Plotting Code ........................................179

APPENDIX H: Full Pixhawk Parameter List ...................................................183 


\section{LIST OF TABLES}

Table

Page

1. Table of requirements for the design of new severe weather UAS .....................................17

2. List of wind, gust, and rain category metrics defined for this project.....................................17

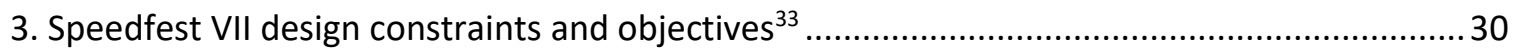

4. Tracking of PWM signals through each subsystem at stock and final parameters ..................72

5. Break test data comparing strength of $1 / 8^{\prime \prime}$ Divinicell foam to $1 / 16^{\prime \prime}$ balsa .............................87

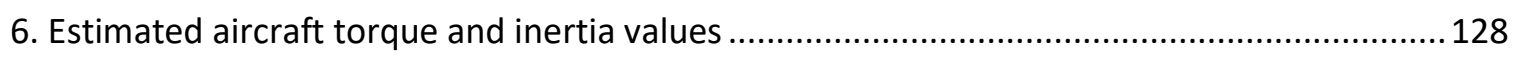

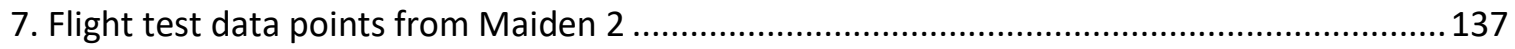

8. Full table of PWM signal pass-through of all avionics systems ............................................ 159

9. Pitch thrust vectoring deflection at each PWM signal value ................................................161

10. Thrust cell data of Kingtech K70G2 turbine used in TIA ................................................... 165

11. Wind loading estimations for the launcher's structure .................................................... 173 


\section{LIST OF FIGURES}

Figure

Page

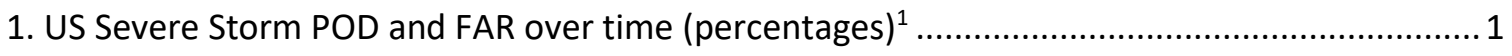

2. US Tornado POD and FAR over time (percentages) ${ }^{1}$............................................................... 2

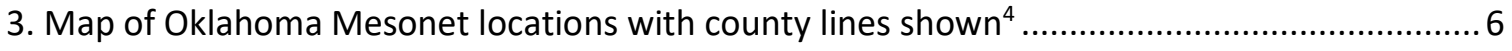

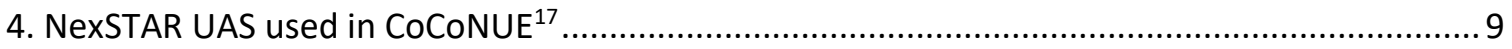

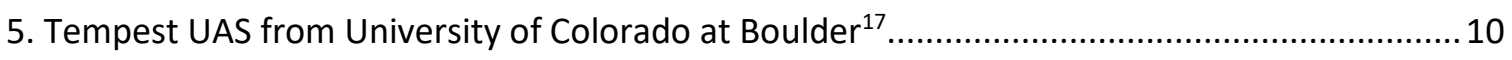

6. FAA COA approval map for Vortex2. Blue is ceiling $=2500 \mathrm{ft}$, Pink ceiling $=400 \mathrm{ft}^{16} \ldots \ldots \ldots \ldots \ldots 11$

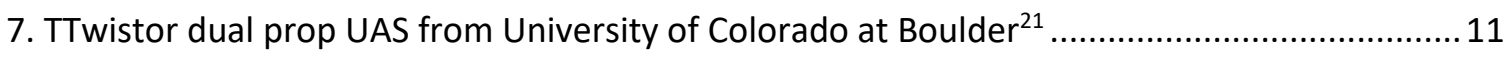

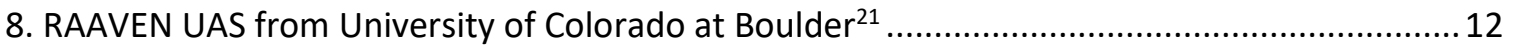

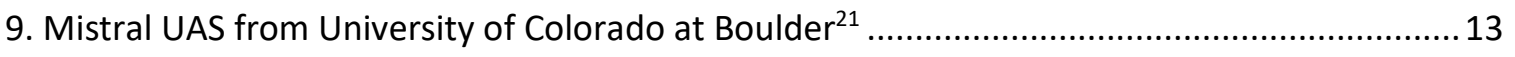

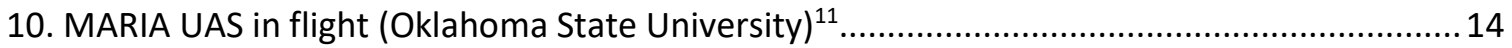

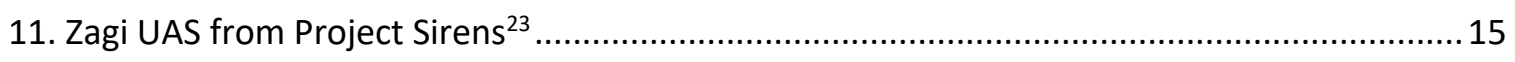

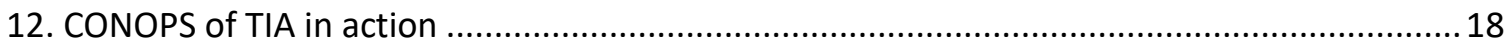

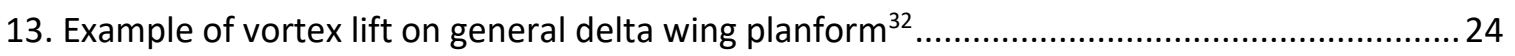

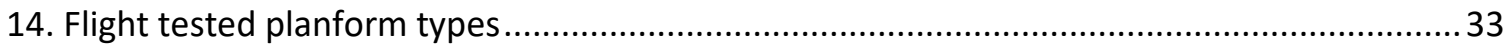

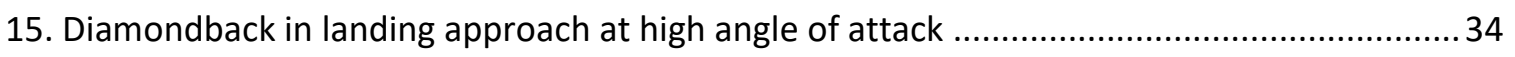

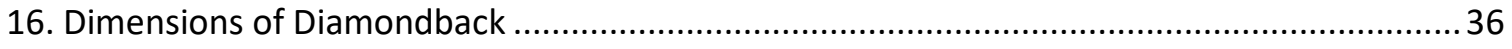

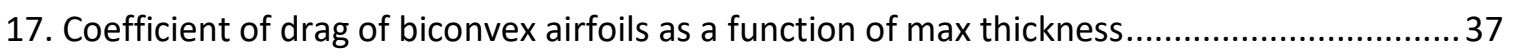

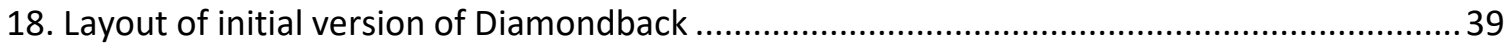

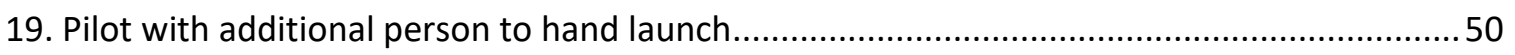

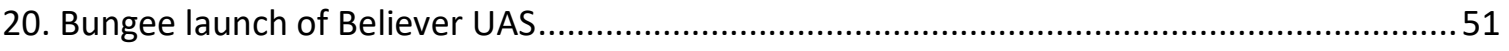

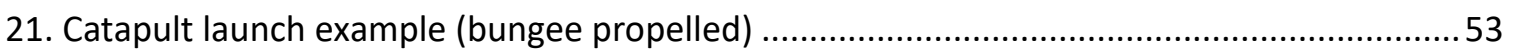

22. Roof-Mounted Cable Release Example (University of Colorado Boulder) ..............................55

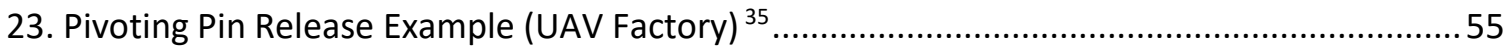

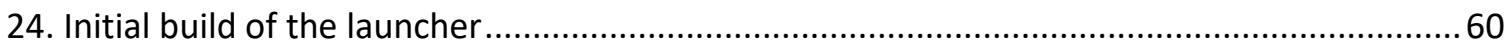

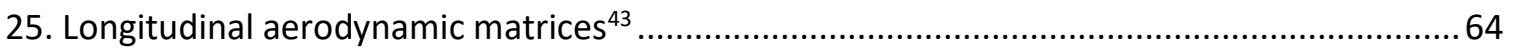

26. Force and moment equations for longitudinal aerodynamic matrix $\operatorname{set}^{43}$.............................64

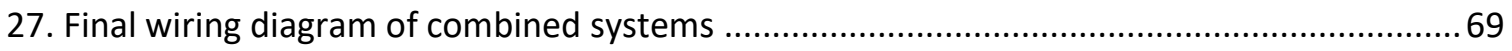

28. Trim tab effect on throttle PWM signal through Pixhawk ................................................... 74

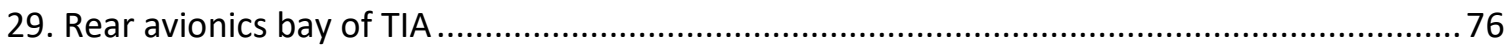


30. Damage to test aircraft from small trees and barbed wire fence at $70+\mathrm{mph}$ due to turbine failure

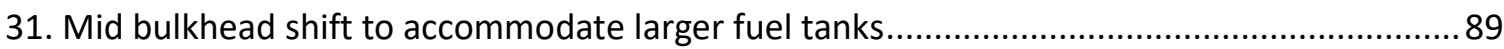

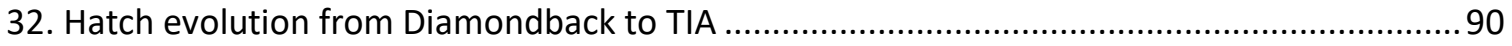

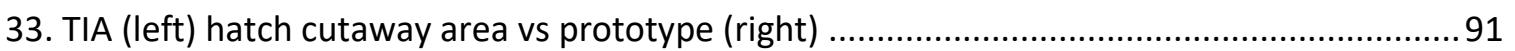

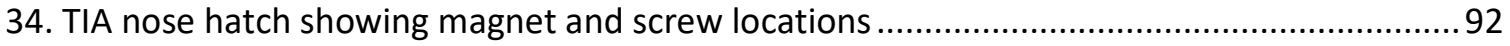

35. Hatches after layup but before removal from mold .........................................................93

36. Test fitting the fuel tanks with the primary structure on a prototype airframe. ....................95

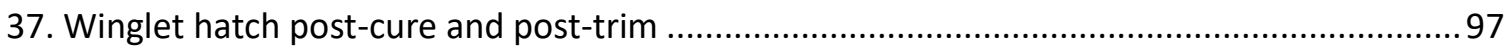

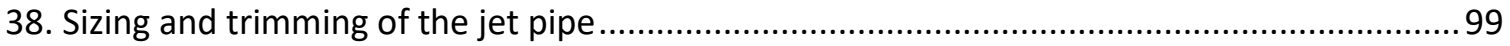

39. Shielded fuel tubing under turbine and secured to skin. Also visible is the launcher

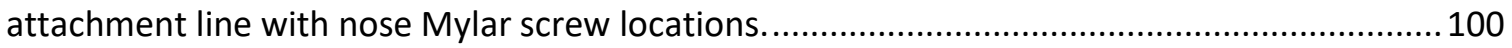

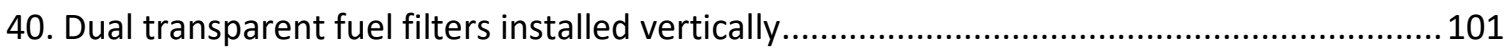

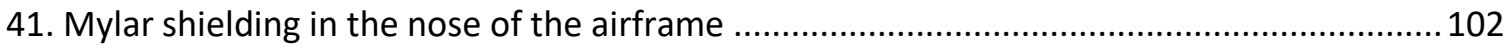

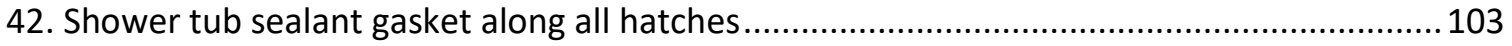

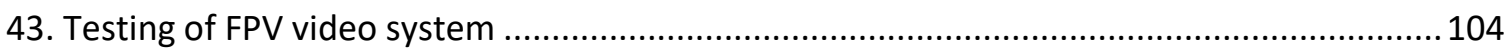

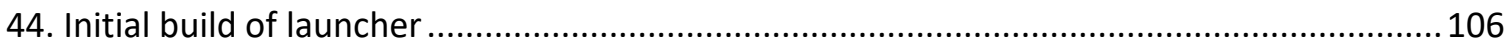

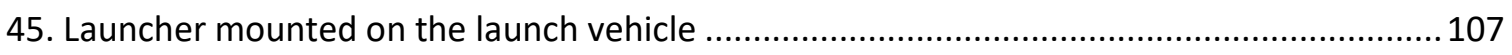

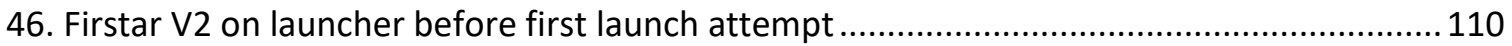

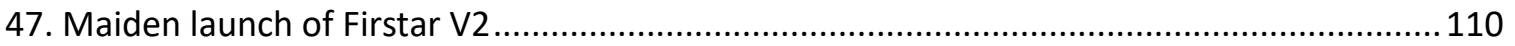

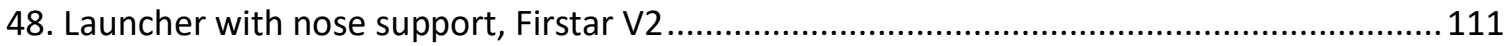

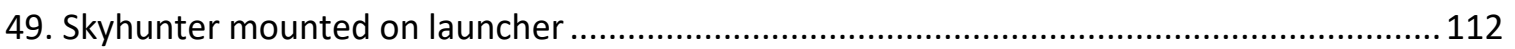



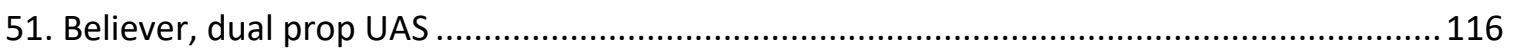

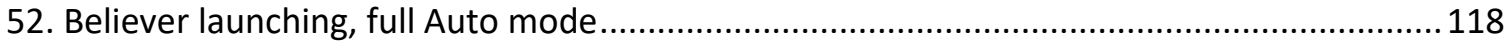

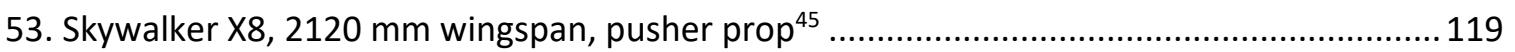

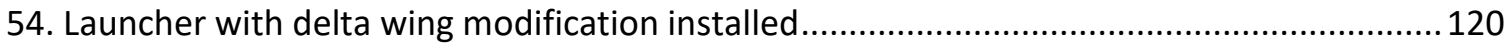

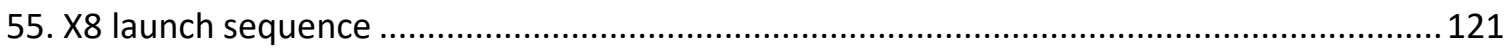

56. All four tested aircraft exhibiting roll on release except for the Believer (lower left) ..........122

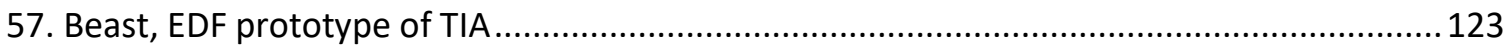

58. Beast on launcher for fine-tuned placement of all support structure..................................125

59. Beast launch, severe roll on takeoff with rapid recovery ....................................................127

60. Roll angles and rates from frame-by-frame video review of launched aircraft ....................129

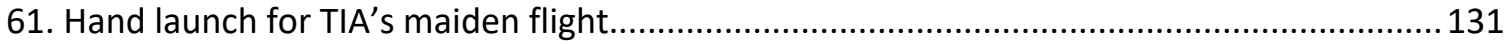

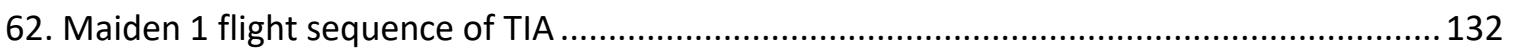

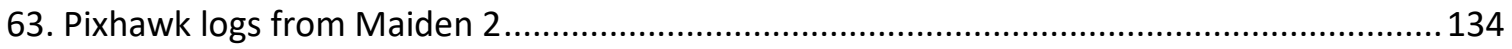

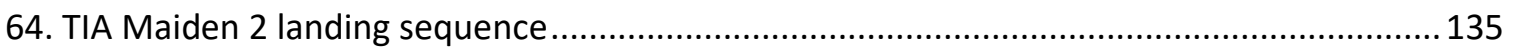

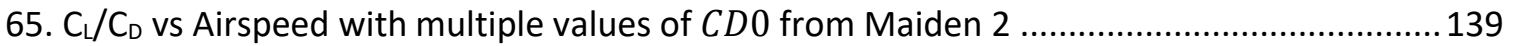

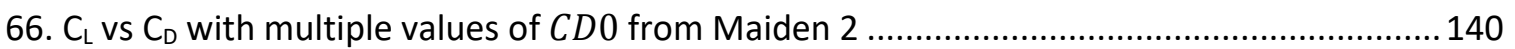

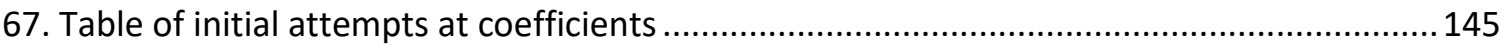


69. Graphical transition from prototype to final product. Blue components were removed or

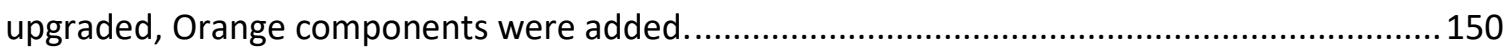

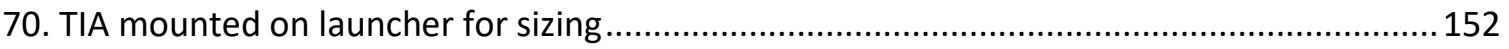

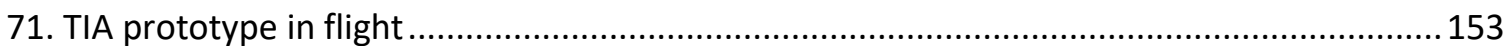

72. Used to measure angular deflections recorded in Table 8 ................................................160

73. Used to measure pitch thrust vectoring angular deflections recorded in Table 9 .................160

74. Test matrix of inlet shape and location used for K45 sizing on prototype ............................. 162

75. Thrust vs inlet area for Kingtech $\mathrm{K} 45$ with no jet pipe ${ }^{24}$. Jet pipe increases required inlet area,

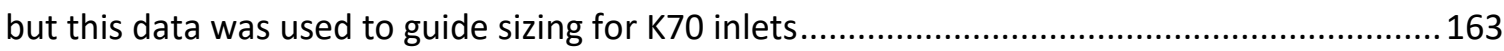

76. Baseline thrust metrics: open chamber with no flow restriction ........................................163

77. Single inlet ( $6.3 \mathrm{in}^{2}$ inlet area) above turbine. Turbine is choked ........................................ 164

78. Dual inlets ( $9.8 \mathrm{in}^{2}$ total inlet area) above turbine. Better performance, slightly exceeds

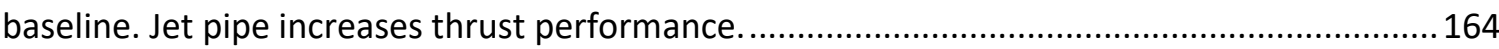

79. Single inlet (12 in ${ }^{2}$ inlet area) above turbine. Best performing inlet choice, was used for

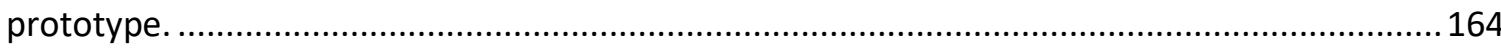

80. K70G2 turbine used on TIA mounted to thrust cell undergoing calibration .........................165

81. Rear inlet area: 9.7in ${ }^{2}$ open area around jet pipe. Blocked front inlets, pure reverse flow in

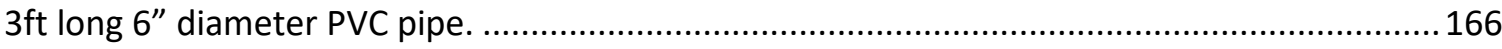

82. Rear inlet area: $25.4 \mathrm{in}^{2}$ open area around jet pipe. Blocked front inlets, pure reverse flow in

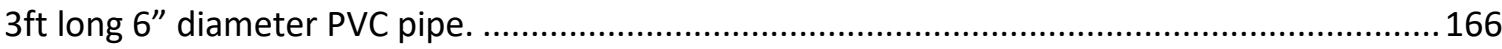

83. Fuel tanks are non-removable, they are installed during airframe manufacturing ..............167

84. Top skin after layup but before removal. Hatches can be seen under skin ..........................168

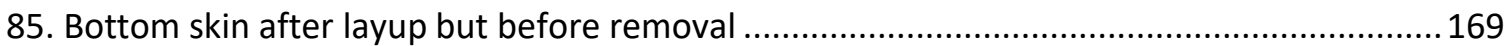

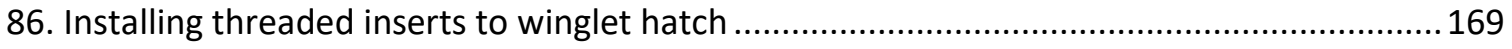

87. Airframe after bonding halves together (winglets uninstalled) ..........................................170

88. Elevon hinge line showing holes for servo linkage pass-through ........................................170

89. Aft view from the nose of the aircraft prior to component installation................................171

90. Rear starboard avionics bay prior to component installation. .........................................172

91. Test matrix of winglets from Speedfest. The $68 \mathrm{in}^{2}$ winglets had optimal performance for its

size, with winglets outperforming a single vertical tail in high AoA landings............................172

92. Graphical comparison of Biconvex, NACA 0010-35, and a Modified NACA 0010-35 airfoils 174

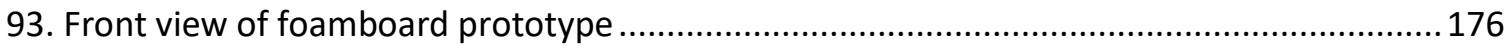

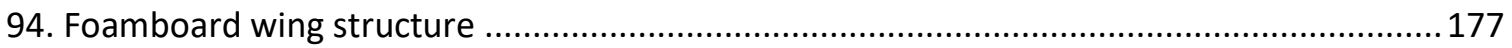

95. Final foamboard model that simulates full composite airframe .........................................178 


\section{CHAPTER I}

\section{INTRODUCTION}

\subsection{Motivation}

Every year, severe weather threatens the lives of many across the United States and the world. Great strides have been made in recent years to aid in correctly predicting and properly warning residents of potential severe thunderstorms, as the False Alarm Rate (FAR) is at $48 \%$ with a Probability of Detection (POD) of $81 \%$ as of $2013 .{ }^{1}$ However, when these predictions extend to include tornado statistics, the FAR increases to $74 \%$ and the POD decreases to $57 \% .^{1}$ As noted in Figure 1 below, the severe storm prediction has leveled out with current prediction methods.

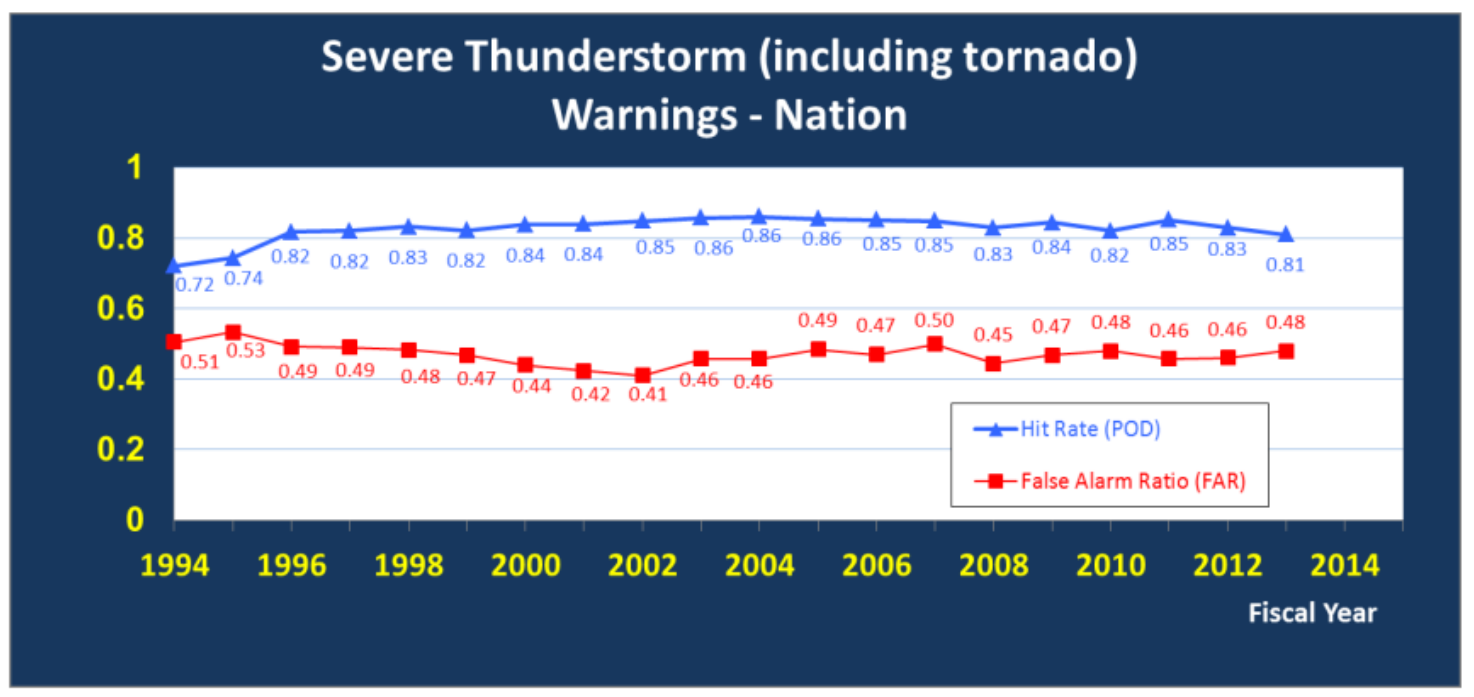

Figure 1: US Severe Storm POD and FAR over time (percentages) ${ }^{1}$ 
The tornado predictions, however, are on a downward trend despite the use of modern meteorological equipment and techniques (Doppler radar, supercomputer-aided weather model forecasting, etc.), as can be seen in Figure 2.

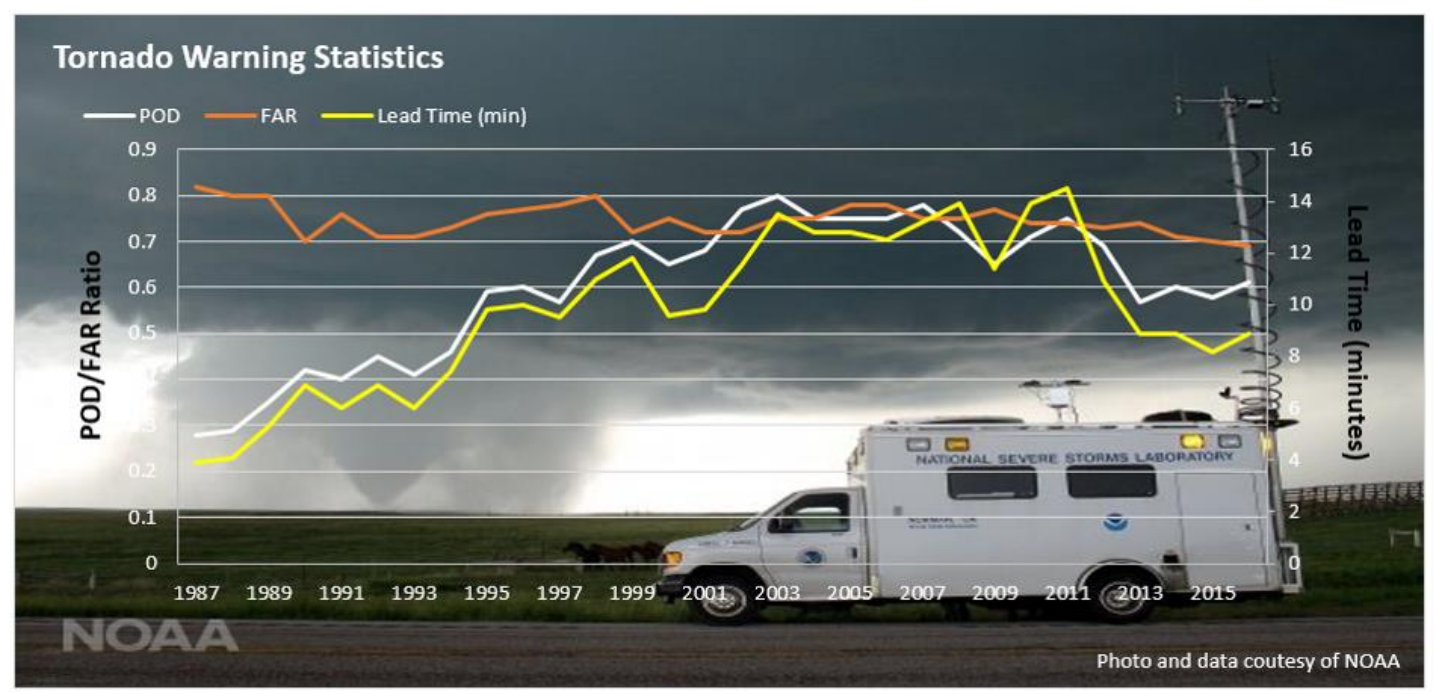

Figure 2: US Tornado POD and FAR over time (percentages) ${ }^{1}$

Just as the POD has been decreasing in recent years for tornado predictions, so has the lead time. This is due in part to the change of how the NWS issues warnings. Until 2007 warnings were issued county-wide. Starting in October 2007 (start of Fiscal year 2008), warnings were no longer issued for an entire county, but rather are "now issued and verified solely for the areas impacted by the warning and event", which reduced the warning area from multi-county warnings to areas the size of a large city or small county ${ }^{2}$. This reduced target area is partly why the POD trend has turned downhill, as meteorologists now are required to make more accurate predictions. This also has led to lower lead times, as the meteorologists attempt to reduce the FAR by delaying the warnings until they are reasonably confident of tornado formation. Though this new facet is added, it does not eliminate the underlying issues with why the success rate is so relatively low and the FAR is large. 
The inability to reliably predict tornado genesis is largely due to the lack of vital in-situ thermodynamic data that predicates the formation of a tornado ${ }^{3}$. If meteorologists and atmospheric scientists had data that showed specific conditions during the formation and movement of these severe weather phenomena, then less false alarms would be issued to residents.

\subsection{Background}

In modern weather forecasting, the majority of atmospheric sensing is centered on the upper atmosphere using Doppler radar, weather balloons, and in some cases manned aircraft. Oklahoma provides a unique opportunity for weather data acquisition in that there are a total of 120 Mesonet sites (10m towers gathering temperature, pressure, humidity, wind speed, wind direction) scattered across the state's 77 counties, with at least one located in each county ${ }^{4}$. Though this provides a high resolution of ground level data, there still exists a void in the data collected for the lower atmosphere (100 feet-15,000 feet). In this void exists a particular weather phenomenon that causes much damage to property and loss of life each year: tornados. Currently, there is not a feasible method of getting reliable data from inside a tornadic system.

Some Unmanned Aerial Systems (UAS) have been used in the past to help fill this atmospheric data gap, but in the specific area of severe weather data acquisition, the generally used remote sensing platforms (quadcopters, electric fixed wing UAVs) tend to fail due to aircraft limitations. These aircraft are relatively cheap $(<\$ 1000)$, and can carry many sensing systems to accurately measure specific parameters in this data gap. Though they are the workhorse remote sensing platforms for non-severe weather, these aircraft that are susceptible to failure (without modification) are so due to: rain (quadcopters, electric fixed wing), high wind gusts (quadcopters, 
lightweight fixed wings), and BVLOS-capable flight requirement with high endurance and top high speed (quadcopters, electric aircraft).

\subsection{Goals and Objectives}

With the shortcomings of the general remote sensing platform in mind, there arises a need for a small Unmanned Aerial System (sUAS) that can accomplish each of the above tasks: operate in actively precipitating, high gust environments while able to maintain sufficient airspeed to reach a target phenomenon in a short time span and remain on target long enough to get useful data. This project should be within the following design constraints:

- Deployable from a vehicle with a small team

- Operate in any environment (high gust, high rain, etc.)

- Reach a high top speed to reach a target phenomenon before it dissipates

- Maintain suitable cruise speed to stay on target long enough to get pertinent data

- Withstand light debris and hail

The goal is to produce an aircraft that can launch from anywhere, gather atmospheric data, transmit the live data down to operators on the ground, and be able to land safely in any terrain.

\subsection{Thesis Outline}

As mentioned previously, there have been other attempts to gather this same type of data as well as similar data sets. These systems will be reviewed, and expectations for an aircraft to gather data near severe storm events will be discussed. The sensor requirements for atmospheric 
measurements are also discussed. The aircraft design is discussed at length with regard to the developed requirements for a severe storm UAS. The necessary modifications and redesign are fully described, including the build process of a custom composite airframe. The extensive flight testing, including problems with integrating the propulsion system with an autopilot, are explained along with the solutions. The calibration and validation of the sensors integrated into the aircraft are also discussed, as well as methods to quickly execute these routine tests in the field. The paper finishes with final thoughts and plans for future work. 


\section{CHAPTER II}

\section{LITERATURE REVIEW}

\subsection{Current Atmospheric Sampling Methods}

Modern meteorologists use a variety of sensing instruments and platforms to feed their computer models and give the most accurate predictions to the public as possible. These sensing systems include: ground based sensing towers, weather balloons, radar, and manned aircraft. For example, across Oklahoma is a network of 108 sensing towers that are a part of the Oklahoma Mesonet ${ }^{4}$. The towers are 10 meters high with the goal of tracking a variety of information: wind speed, wind direction, air temperature, humidity, solar radiation, pressure, and rain accumulation (total and rate), among others.

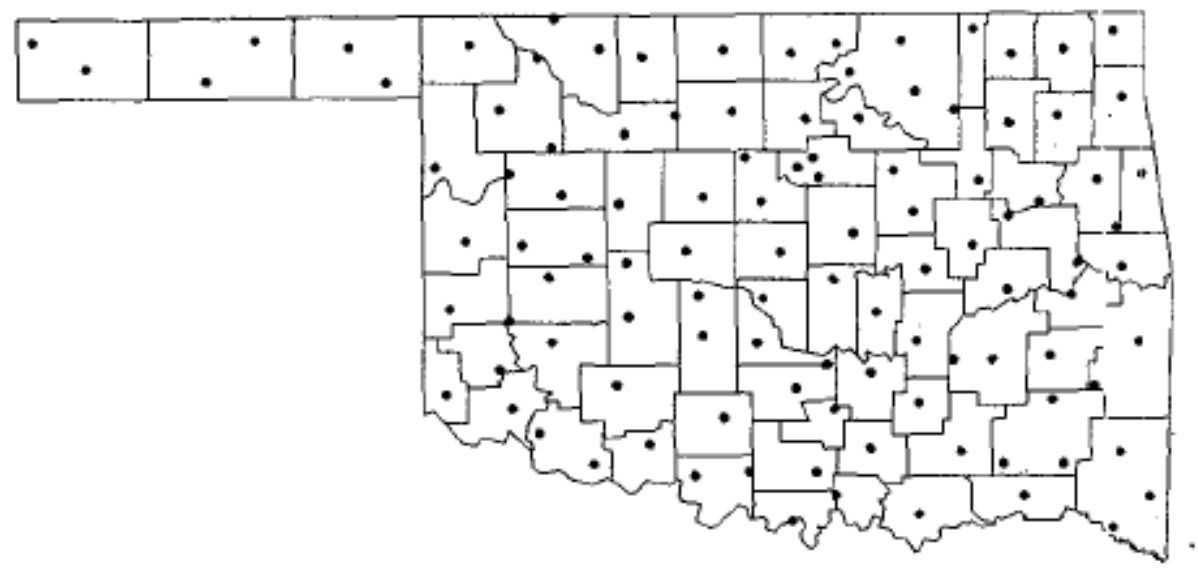

Figure 3: Map of Oklahoma Mesonet locations with county lines shown ${ }^{4}$ 
Weather balloons are another commonly used atmospheric measuring system that utilizes Radiosondes to capture in-situ atmospheric data. These sensors gather information in set intervals as the balloon ascends through the atmosphere while relaying the data back down to the ground ${ }^{5}$. These instruments typically travel $30 \mathrm{~km}$ AGL while measuring pressure, humidity, temperature, wind speed, and wind direction (Radiosonde SOURCE). Though these sensors are typically launched twice daily from each predetermined site at the same time globally (0000 and 1200 UTC), launches at 0600 or 1800 are not uncommon if a severe or other notable atmospheric system was nearby. While this data is sufficient for trends and general trajectories, this data alone is not sufficient for severe storm predictions or monitoring.

Great strides in Doppler Radar have been made to help fill in data gaps and to observe how atmospheric systems move and interact. The National Weather Service (NWS) upgraded all their radar stations starting in the early 1990s and ending in the early 2000s through project NEXRAD (NEXt-generation RADar). This implementation improved the resolution and accuracy of the radar stations in regards to severe weather (tornados in particular, see Figure 2), which allowed for meteorologists and atmospheric scientists to make more precise predictions ${ }^{6}$.

Despite the best efforts of these various systems, there is still a lot left to be desired in terms of atmospheric predictions. The driving reasoning is the radars cannot directly observe thermodynamic interactions in a storm, weather balloons rise through target areas too rapidly for small-scale measurements, and stationary towers are too short to reach the needed data sets. As such, there is a need to find this in-situ thermodynamic data that currently has been unobserved.

\subsection{Data Capture from Aircraft}

Manned aircraft have also been used to actively collect thermodynamic and other atmospheric data near severe weather, but these approaches are used sparingly they are much more expensive 
to sustain for long periods of time, and the risk to loss of life is substantially increased over other methods. One such proposed approach includes the A-10 Warthog that was to be outfitted with extra shielding and a sensor suite to penetrate thunderstorms and other sever weather systems ${ }^{7}$. This project involved taking a retired A-10, refitting it with extra lightning and debris shielding, removing all military armaments, and adding in civilian equipment as well as ballast. Due the technical challenges involved with this project, there were many long delays in the project that resulted in a very large cost requirement, and the project was eventually halted. The aircraft currently sits in a hangar at the National Center for Atmospheric Research in Colorado.

A manned project that actually took flight was the Tropical Cyclone Structure-2008 with a goal to increase predictability of tropical cyclones in the western North Pacific region ${ }^{8}$. This project involved both a Navy Research Lab NP-3 and two Air Force Reserve WC-130J aircraft. The NP3 utilized deployable dropsondes and ELDORA radar to map the internal structure of the storms. The WC-130s also utilized dropsondes and a full sensor suite on board the aircraft. The primary metrics tracked by the aircraft were wind speed, wind direction, humidity, and temperature while the dropsondes were capturing wind speed, wind direction, humidity, and barometric pressure to generate vertical atmospheric profiles.

From the shortcomings of conventional atmospheric sensing (balloons, towers, and radar) as well as the relatively high costs and risks associated with manned observations, there has been a growing interest in using Unmanned Aerial Systems (UAS) to gather in-situ measurements ${ }^{9-16}$. Since the mid 2000's, researchers at the University of Colorado-Boulder and University of Nebraska-Lincoln have been testing the feasibility of using UAS platforms to gather meteorological data from moving weather systems in a variety of projects ${ }^{10,12-14}$.

The first of these projects was CoCoNUE: Collaborative Colorado-Nebraska Unmanned Aircraft System Experiment, which took place from March 1 to September 30 of $2009^{12}$. The goal of this project was to determine the feasibility of using unmanned aircraft to collect in-situ data 
from moving cold fronts and thunderstorm-generated gust fronts. This was conducted using a NexSTAR UAS from Hobbico, which is a lightweight aircraft with Monokote skin, balsa airframe, and plywood internal structure with a glow-fuel-powered motor for propulsion.

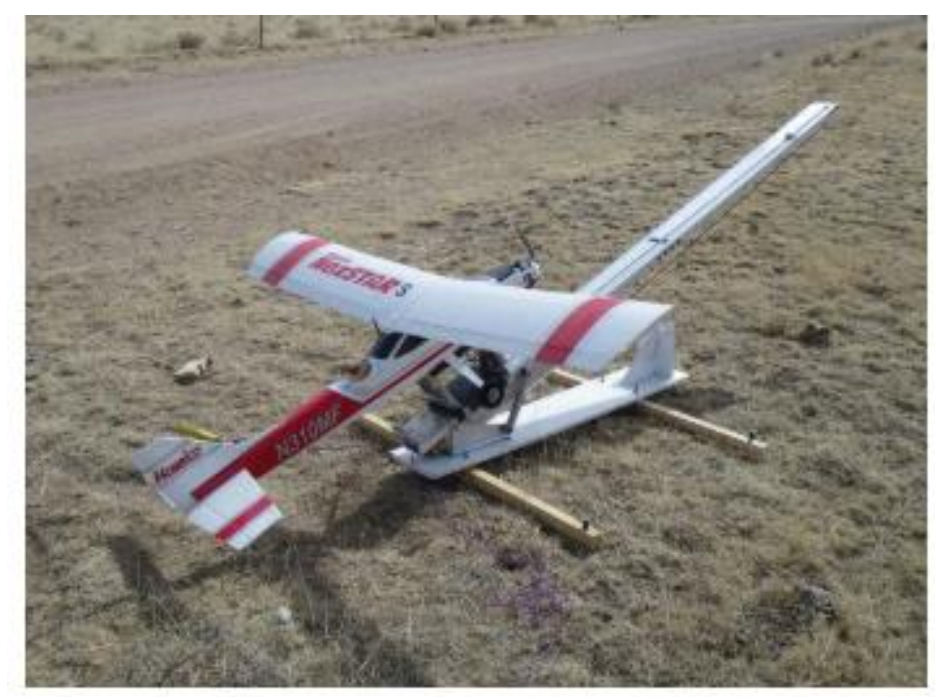

Figure 4: NexSTAR UAS used in CoCoNUE ${ }^{17}$

This aircraft was deployed from a mobile team using a launcher (as shown above) with instrumentation on board to gather atmospheric data. From this testing, general guidelines for data captured were developed, which were directly used in follow-on projects ${ }^{9}$.

The next of these projects was in May-June of both 2008 and 2009: Vortex2, a follow on project to Vortex, which was a project in the mid 1990's that resulted in ground-breaking data collection of the life cycle of tornadoes ${ }^{18}$. For Vortex 2 , the researchers increased the number of sensors and platforms to gather as much thermodynamic data as possible to attempt to determine the factors that cause tornadogenesis ${ }^{14}$. Among these additional platforms were UAS. Some of the major hurdles faced were FAA airspace regulations as well as maintaining command and control of the aircraft while flying Beyond Visual Line of Sight (BVLoS). This required much collaboration with the FAA and systems checks, but in the end resulted in multiple flights through the Rear Flank Gust Front (RFGF) and Rear Flank Downdraft (RFD) of multiple supercells, showing the systems are capable. ${ }^{10}$ 




Figure 5: Tempest UAS from University of Colorado at Boulder ${ }^{17}$

The Tempest UAS, shown above, was designed as a tornado chasing aircraft (note: chasing, not penetrating). It was outfitted with a Piccolo autopilot system and the same sensors used on the NexSTAR for CoCoNUE. This aircraft is a full composite airframe, with a wingspan of $10.5 \mathrm{ft}$, endurance of 2-4 hours, top speed of $78 \mathrm{mph}$, and GTOW of 15 pounds ${ }^{19}$.

More recently, the same group received approval from the FAA for UAS flights over much of the Great Plains, as can be seen in the below figure. The blue represents a ceiling of $2500 \mathrm{ft}$, and the pink a ceiling of $400 \mathrm{ft}$. This is a significant feat of its own, as it is the first time UAS have been granted a multi-state operational area with a $2500 \mathrm{ft}$ ceiling across the entire region, which will likely be a stepping stone for future use of UAS for atmospheric measurements ${ }^{16}$. As of this writing, the project is still ongoing, with the second set of field campaigns starting in the Spring of 2020 . 


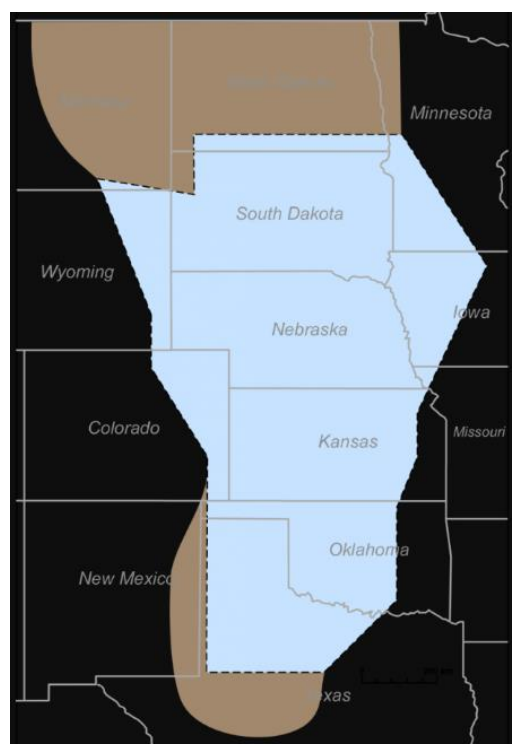

Figure 6: FAA COA approval map for Vortex2. Blue is ceiling $=2500 \mathrm{ft}$, Pink ceiling $=400 \mathrm{ft}^{16}$

This project is much more ambitious than previous in-situ observation attempts, as this time NOAA's P-3 Hurricane Hunter would be used for radar observations from the air coupled with ground-based radar arrays to map the internal structure, as well as UAS and multiple weather balloon launches to gather thermodynamic data throughout the system ${ }^{20}$.

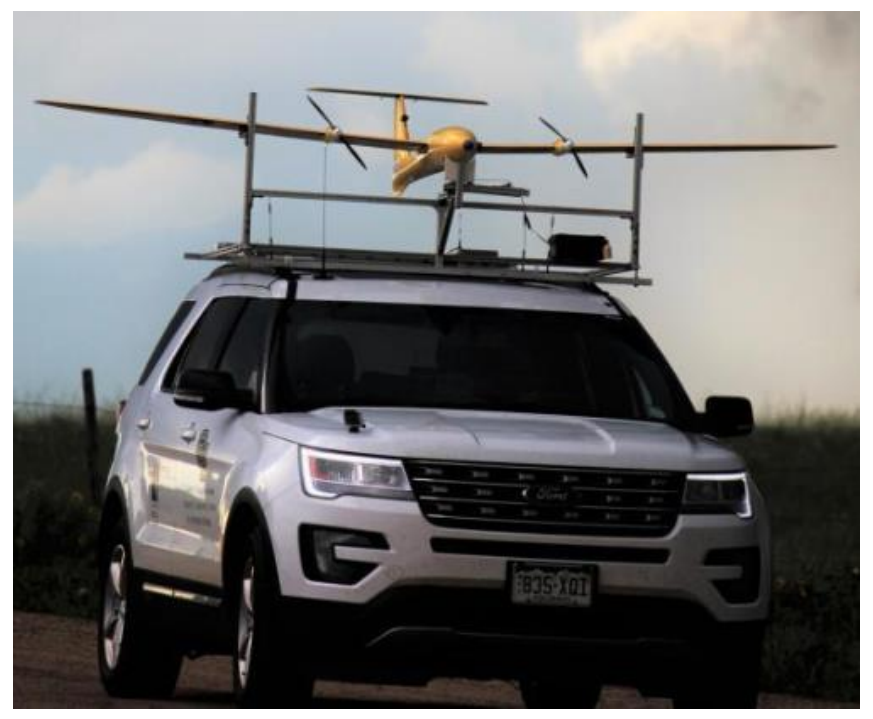

Figure 7: TTwistor dual prop UAS from University of Colorado at Boulder ${ }^{21}$ 
The primary UAS used by UC Boulder for TORUS was their custom TTwistor UAS (a dual prop version of the Tempest), which featured a $10.5 \mathrm{ft}$ wingspan, endurance of 3 hours, top speed of 95 mph, and GTOW of 20 pounds ${ }^{21}$. This aircraft was used in conjunction with their RAAVEN aircraft.

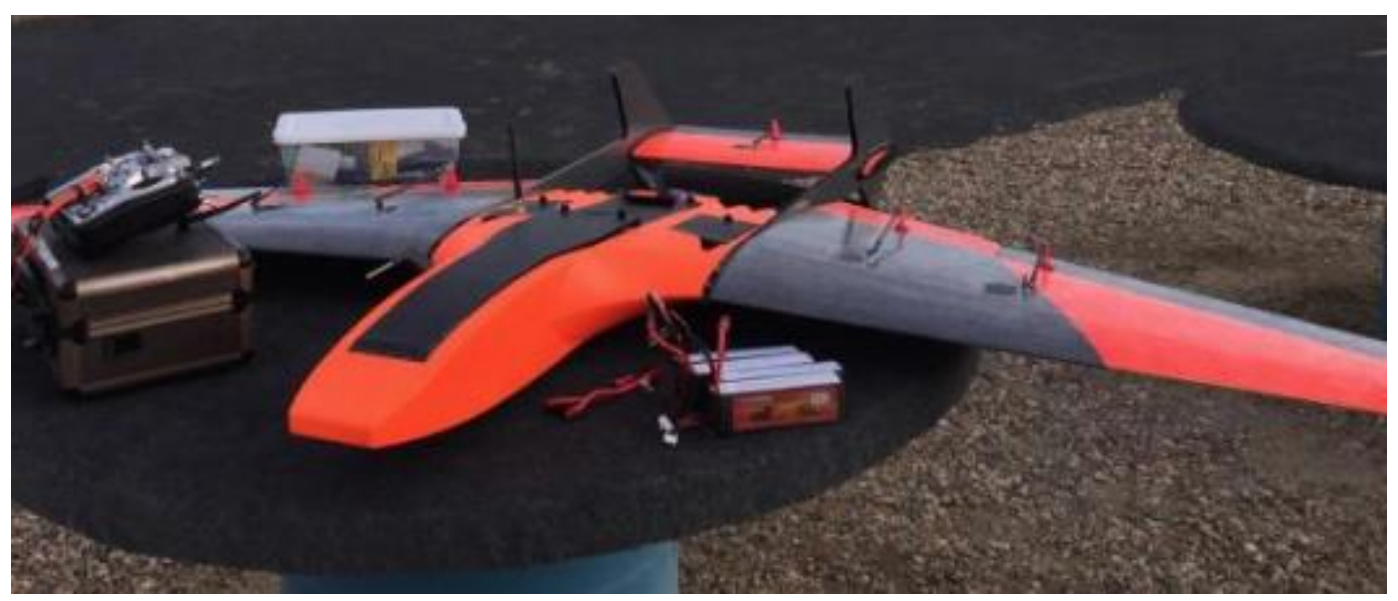

Figure 8: RAAVEN UAS from University of Colorado at Boulder ${ }^{21}$

RAAVEN is an airframe built from EPP foam, which allows it to be a very lightweight airframe.

This aircraft has a wingspan of $7.6 \mathrm{ft}$, endurance of 2 hours, top speed of $80 \mathrm{mph}$, and a GTOW of 3.8 pounds $^{22}$. This aircraft was flown alongside two other RAAVENs for a coordinated observations of the moving atmospheric systems.

The team at UC Boulder have also developed a successor to the TTwisot: Mistral. This aircraft builds on the success of TTwistor and Tempest, keeping the overall geometry similar by expanding for longer endurance missions. Though it hasn't flown for a project yet, that is their goal to get it ready for use in the field. 


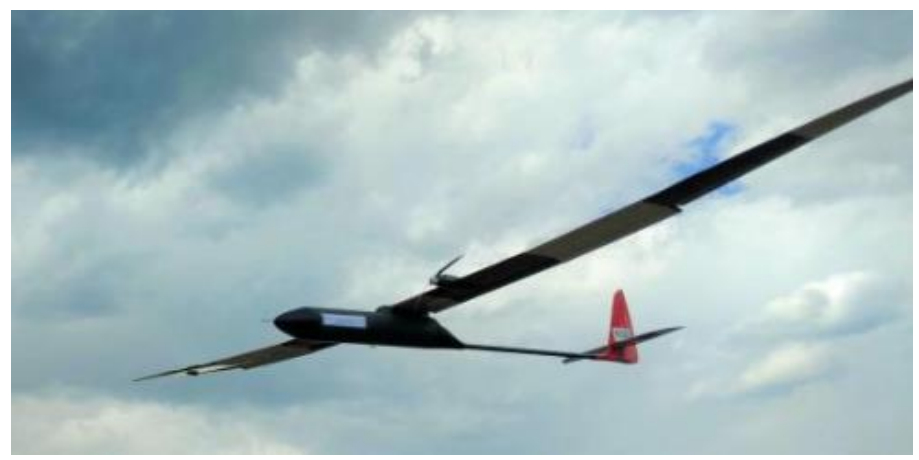

Figure 9: Mistral UAS from University of Colorado at Boulder ${ }^{21}$

The Mistral utilizes a dual prop propulsion system seen in the TTwistor, but increases the wingspan to accompany the increased endurance. The wingspan is $16 \mathrm{ft}$, endurance of 6 hours, top speed of $100 \mathrm{mph}$, and GTOW of 40 pounds $^{21}$.

An aircraft was also previously developed at Oklahoma State with a similar goal as these other severe weather UAS, which was to penetrate through super cell systems while gathering insitu thermodynamic data for prolonged periods of time. This aircraft, named MARIA, utilizes a bomb-bay door to deploy dropsondes as targeted points along the flight path, allowing for additional vertical profiles of data to compliment the in-flight data gathered along the flight path. ${ }^{11}$ 


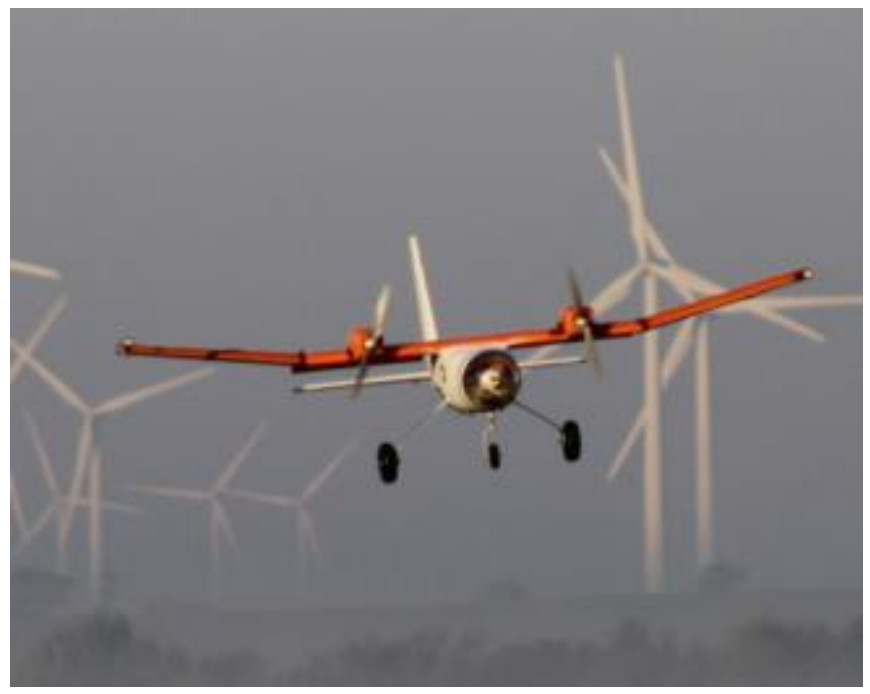

Figure 10: MARIA UAS in flight (Oklahoma State University) ${ }^{11}$

MARIA has a wingspan of $7 \mathrm{ft}$, endurance of 8 hours, top speed of $126 \mathrm{mph}$ (110 kts), and a GTOW of 35 pounds. Much like Tempest and Mistral, MARIA uses a dual-prop propulsive system; however, MARIA was intended to use gas-powered motors rather than all-electric due to the higher energy density of gasoline. Currently, MARIA still uses a full-electric system.

A crow-funded project, named Project Sirens, was attempted in Alabama in 2014. Though this project eventually failed due to insufficient funds, the attempted goal was to fly a delta wing UAS through a tornadic system to gather in-situ thermodynamic data ${ }^{23}$. For prototyping, a modified Zagi HP60 was used. 


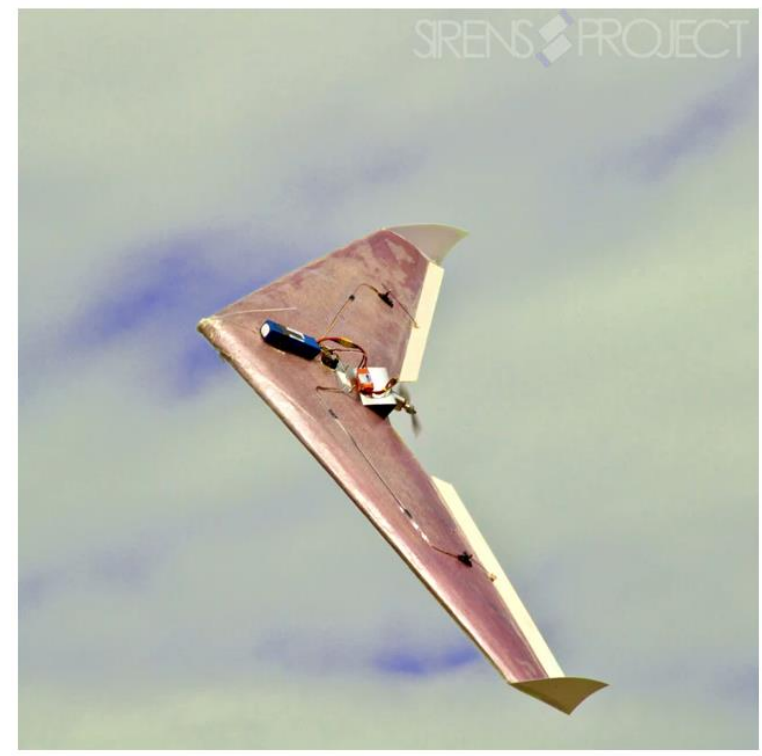

Figure 11: Zagi UAS from Project Sirens ${ }^{23}$

The Zagi HP60 has a wingspan of $5 \mathrm{ft}$, top speed of $55 \mathrm{mph}$, and airframe weight of 2 pounds (before payload). This aircraft was to be used as a demonstrator before building a custom composite airframe of similar dimensions. However, due to failing to reach funding limits, the project never left the prototyping phase.

\subsection{Takeaways for Aircraft Development}

The most pertinent data gathered from the previous flight campaigns is the average winds experienced, as strong winds and gusts present a major structural issue to any airframe. The Tempest UAS, developed by University of Colorado at Boulder, was the first to attempt to penetrate super cell thunderstorms, and across as such has now flown in many supercell system. Across all flights, the average winds across all supercell intercepts was $11.2 \mathrm{~m} / \mathrm{s}(25 \mathrm{mph})$ with a $\max$ of $26.2 \mathrm{~m} / \mathrm{s}(58.6 \mathrm{mph}){ }^{10}$. Of note is the mission path: the aircraft is primarily penetrating through the Rear Flank Gust Front (RFGF) to reach the Rear Flank Downdraft (RFD) before 
coming back outside of the RFGF. This was repeated at multiple altitudes across all missions, measuring temperature and humidity. As this is one area of interest, this data is very useful in ensuring the aircraft is capable of sampling. While this location is a growing location of interest for in-situ thermodynamic sampling, there are still limitations for current aircraft to get closer to the core of the system. The gust fronts of supercell thunderstorms can have straight line winds resulting from the downdraft fronts attaining $60 \mathrm{mph}$ on average with max speeds of $100 \mathrm{mph}^{25}$. From these same systems, tornados can be born, with windspeeds up to the strength of the tornado itself (65 mph for EF0 with 200+ for EF5) ${ }^{26}$. These data points are important, as approaching near a tornadic system will require a high level of robustness to withstand those high wind speed intensities, especially if they are variable rather than straight line. 


\section{CHAPTER III}

\section{DESIGN REQUIREMENTS AND CONCEPTS}

\subsection{Design Requirements and CONOPS}

Using what was gleaned from the Lit Review, several parameters stood out as the main operating points to target with this aircraft. Some noteworthy ones are:

\begin{tabular}{|c|c|c|}
\hline Requirement & Objective & Threshold \\
\hline Operate in precipitation & Tier 3 & Tier 2 \\
\hline Operate in high winds & Tier 3 & Tier 2 \\
\hline Sufficient top speed to rapidly reach target & $130 \mathrm{mph}$ & $100 \mathrm{mph}$ \\
\hline Sufficient cruise for data acquisition (60 mph max) & 60 & 40 \\
\hline Sufficient endurance for mission & 20 minutes & 15 minutes \\
\hline Withstand light debris and hail (random chance) & Yes & Yes \\
\hline Rapidly deploy from vehicle & Less than 5 minutes & Less than 10 minutes \\
\hline Operate with small team & 3 or fewer & 5 or fewer \\
\hline Capable of flying over long range & Autopilot \& RC & Pure RC \\
\hline FPV system for long range situational awareness & Yes & Yes \\
\hline Safely land near personell & Land anywhere & Land on road, flat grass \\
\hline
\end{tabular}

Table 1: Table of requirements for the design of new severe weather UAS

Where the ambient wind speeds, gusts, and precipitation rate tiers are defined below:

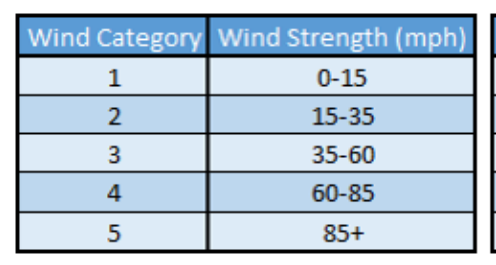

\begin{tabular}{|c|c|}
\hline Gust Category & Gust Strength $(\mathrm{mph})$ \\
\hline 1 & $0-10$ \\
\hline 2 & $10-25$ \\
\hline 3 & $25-40$ \\
\hline 4 & $40-60$ \\
\hline 5 & $60+$ \\
\hline
\end{tabular}

\begin{tabular}{|c|c|}
\hline Rain Category & Rain Accumulation (per hour) \\
\hline 1 & Trace (greater than $0^{\prime \prime}$ ) \\
\hline 2 & Light (up to $0.1^{\prime \prime}$ ) \\
\hline 3 & Moderate (0.1"-0.3") \\
\hline 4 & Heavy (0.3" or more) \\
\hline
\end{tabular}

Table 2: List of wind, gust, and rain category metrics defined for this project 
These requirements are derived directly from previous attempts to gather similar data types under similar environmental circumstances and from the target mission hoped to be achieved by the aircraft described in this paper. This target mission can be generally described by the Concept of Operations (CONOPS), which is shown below:

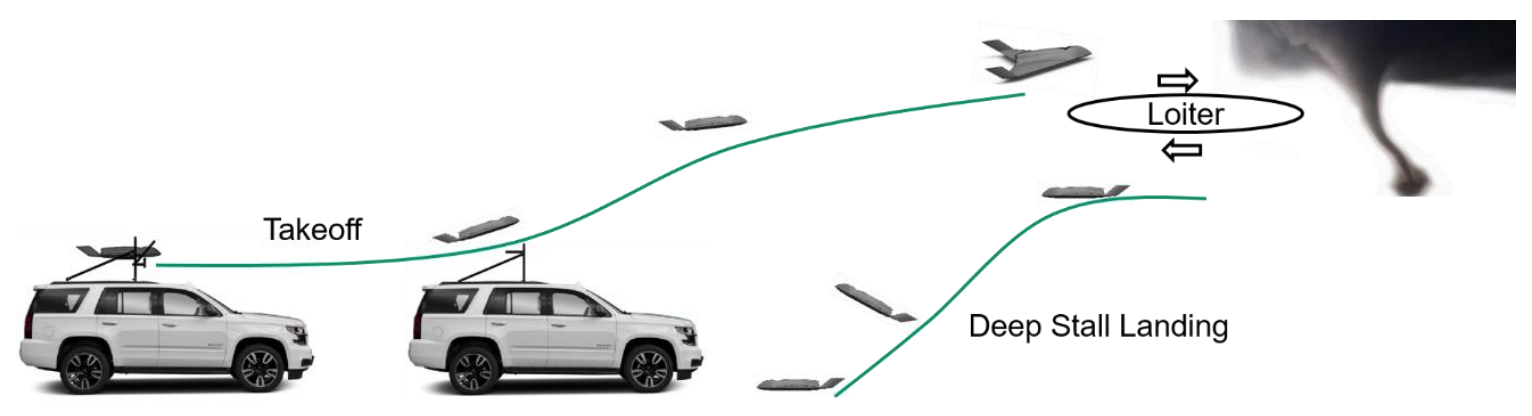

Figure 12: CONOPS of TIA in action

The goal of the aircraft is to launch from a moving vehicle (mobile operations), fly at high speeds to reach the target rapidly, loiter at sufficiently low speeds to gather data, then return to where the operators are (could be same or different than launch point) to execute a high angle of attack/deep stall landing for recovery. The following sections describe the steps taken in the initial design of the aircraft capable of conducted these tasks.

\subsection{Aircraft Characteristic Down-selection}

\subsubsection{Planform Type}

When observing the sUAS that are generally used for meteorological data acquisition, there are two broad categories of aircraft: rotary wing and fixed wing. Rotary wing aircraft work great for vertical profiling of a single location, as they have the capability of ascending nearlycompletely vertically. This means they do not need a runway of any kind for takeoff or landing, only a flat surface where nothing impacts the propellers, and only a single pilot to monitor and fly 
the aircraft. However, most are not capable of withstanding active precipitation. Some companies are beginning to come out with new water-proof rotary wing aircraft but they are still susceptible to the rotary wing platform's primary drawback: inability to withstand high winds and gusts. Typical rotary wings (DJI M100 for example) are not recommended to fly in winds above 20 $\mathrm{mph}$, though they are technically capable of a top speed of up to $50 \mathrm{mph}^{27}$. However, this brings about the other major pitfall all rotary wing aircraft face: short battery life and subsequent short range flights. The DJI M100 has a max rated flight time of 40 minutes, but that's while hovering in ideal conditions with two batteries installed ( 23 minutes with 1 battery). From flight testing, the max flight time for a normal flight on a calm day is 30 minutes, and that drops dramatically I the quad is fighting strong ambient winds. When trying to fly in any form of wind, the motors are having to spin at a much higher RPM to maintain position, which reduces this flight time even further. This, coupled, with a max ground speed of roughly $50 \mathrm{mph}$ and the lack of any form of waterproofing, leaves the aircraft as a relatively slow and short range option for this project. DJI is trying to overcome these drawbacks with the DJI M200, as it is water resistant (IP 43), has a max rated flight time of 38 minutes, and a top speed of $51 \mathrm{mph}$. However, the max rated flight time with a full payload of $2.34 \mathrm{~kg}$ is only 24 minutes $^{28}$. This means the bare quadcopter is nearly capable of use in this area, but when adding on the necessary sensors and payloads, the endurance takes a significant hit. And the rated flight time with payload was still done while hovering in near-ideal conditions, which not be present for many operating environments. When flying in the gusty environment sought after, that flight time will only reduce even further. As such, all rotary wing variants were neglected.

The other group of sUAS used for meteorological data acquisition is fixed wing aircraft. These aircraft are most typically all-electric with either one pusher/puller prop or dual puller props. Inside this grouping are two subcategories: conventional airframes and flying wing airframes. Conventional airframes are defined here as standard tube and wing airframes with 
either a traditional tail empennage or an $\mathrm{H} / \mathrm{V}$ tail on twin booms. Flying wing configurations are usually a single large swept wing with vertical fins for directional stability. Some commonly used flying wing sUAS have vertical fins at the end of the wings, called winglets, for directional stability (Skywalker X8, Opterra) and others use fins offset from the centerline of the fuselage for directional stability (Opterra, RV jet). Each of these categories of aircraft (conventional and swept wing) currently used for meteorological measurements share similar manufacturing processes and payload capacities. These airframes are typically manufactured in an EPO foam, allowing for a lightweight structure that can still withstand a sizeable payload. For example, a standard workhorse conventional airframe sUAS, the Skyhunter, is a foam aircraft, commonly referred to as a foamy, that can easily be outfit with an autopilot, first person view (FPV) video systems, and a sensor suite to gather in-situ meteorological data. The standard metrics tracked from these sensors are temperature, pressure, humidity ${ }^{10-14}$, with many now also approximating ambient wind speed and direction during the flight as well via multi-hole pitot probes ${ }^{29}$. Nearly any fixed wing aircraft being compared here can all hold the same components, so differences between these will not be compared. The primary point of comparison is flight performance, propulsive capability, and structural robustness between these two categories.

Before comparing the aircraft types, it's important to note the frequent use of "typical" and "general" statements that will arise, for a properly designed delta wing can outperform an offdesign conventional airframe in certain circumstances, and vice versa. In generalizing these pros and cons for each airframe type, the best performer for a given mission is always an "it depends" answer, as there is a strong correlation between aircraft performance and the conditions an aircraft is designed to operate in. As such, generalized statements will be made to compare when a conventional tube and wing airframe should perform well versus when it shouldn't, and the same will be made for delta wings. This is not an exhaustive list, nor do all the items hold true in 
all circumstances for all airframes. The validity of each statement is strongly tied to the mission profile the aircraft is flying in versus what it was designed for.

Conventional airframes by nature are the more efficient performing category of the two types of aircraft. Due in part to both the reduced external wetted surface area and the typically higher aspect ratio (AR) wings (relative to delta wings), conventional airframes have the benefit of less skin friction drag than delta wings. Drag can be calculated $\mathrm{as}^{30}$ :

$$
C_{D}=C_{D_{0}}+\frac{C_{L_{0}}^{2}}{\pi * A R * e}
$$

Where $C_{D}$ is the total drag coefficient of the aircraft, $C_{D_{0}}$ is the skin friction and form factor drag coefficient (primarily driven by total wetted surface area and airfoil shape), $C_{L_{0}}$ is the coefficient of lift at $0^{\circ}$ angle of attack, $A R$ is the aspect ratio of the wing, and $e$ is the Oswald Efficiency factor. The rightmost combined term is commonly referred to as induced drag. Aspect ratio can be found $a s^{30}$ :

$$
A R=\frac{b^{2}}{S}
$$

Where $b$ is the wingspan and $S$ is the planform area. From these equations, it's clear that having a large wingspan with smaller overall wing area would generate a high aspect ratio, which in turn reduces the induced drag component of the drag equation. This implies that for a given mission profile, the conventional aircraft will typically have a longer endurance than a delta wing. This can be seen from Endurance and Range Estimation equations (assumptions: constant airspeed, constant lift coefficient, jet propulsion system $)^{30}$ :

$$
\text { Endurance }=\frac{1}{c_{t}} \frac{C_{L}}{C_{D}} \ln \frac{W_{1}}{W_{2}}
$$




$$
\text { Range }=\frac{V}{c_{t}} \frac{C_{L}}{C_{D}} \ln \frac{W_{1}}{W_{2}}
$$

Where $c_{t}$ is thrust specific fuel consumption, $C_{L}$ is coefficient of lift at target condition, $C_{D}$ is total drag coefficient at given condition, $W_{l}$ is weight at start of mission being analyzed, $W_{2}$ is weight at the end of the mission (or next leg of the mission if multi-part), and $V$ is the cruise velocity. Comparing all four equations, if the aspect ratio in EQ 2 is large, that drives the induced drag term in EQ 1 down, reducing overall drag at given flight condition. A reduction in drag results in an increase in both EQs 2 and 4. The opposite is also true, with a lower aspect ratio causing an increased total drag on the aircraft, which results in a decrease in range and endurance estimates. In these categories, conventional airframes typically excel at the cost of a more limited operational regime.

Since these aircraft are tube-and-wing airframes, it also means balancing the aircraft is fairly easy, as the CG should set on the quarter chord of the wing. If the CG is too far forward or aft, a simple movement of an internal component (usually a battery) along the fuselage's length can get the CG balanced properly. On a custom airframe, this gives even more flexibility in the design, as the wing can be shifted forward and backward in the design process before being manufactured. These aircraft are also easier to make statically stable with more intuitive control schemes. Being a tube-and-wing, the fuselage on its own has a directionally stabilizing effect, which can help keep the aircraft pointing into the dominant wind direction with minimal control input. This can be advantageous on takeoff and landing if each are done into the wind (as takeoffs and landings should be done) to help prevent unwanted sideslip that, if left unchecked, could end in disaster.

However, all of these benefits of a conventional airframe come at a cost. Though conventional airframes sport higher aspect ratio wings than delta wings that help increase endurance, they also require a much larger structure factor to maintain the aerodynamic loads 
they are subjected to. For manned aircraft, this increased structure factor (percent of total aircraft weight designated only to structural components) means for a given gross takeoff weight (GTOW), there is less useable weight allotted for payloads or other systems. For large manned transport category aircraft, this is circumvented by mostly gutting the aircraft except the cargo bay while keeping the wings large for a large fuel volume. For common foamy sUAS, neither of these necessarily hold true. Instead, there is typically a single, sometimes a double, spar that is partially inserted into each wing and passes through the fuselage. This means most of the loading is still being held by the EPO foam these aircraft are made from. The same is mostly held true for composite sUAS, but they feature a much stronger skin, which typically allows for larger loading before failure than EPO foam. High aspect ratio wings (which conventional airframes typically utilize) have another large drawback: maneuverability. A high AR wing will have a reduced roll rate when compared to a low AR wing, which can be either good or bad depending on the situation at hand. The reduced roll rate is due to the large moment of inertia a high AR wing carries with it, which can be great at mitigating gust load rolling effects, but the cost is reduced roll control input. For an aircraft that must fly below $400 \mathrm{ft}$ (current FAA restrictions ${ }^{31}$ ), being highly maneuverable is a must to help ensure the aircraft has a high chance of not only avoiding hitting the ground or any structure, but also in returning home in one piece. The large loads these long wings experience while reducing the roll moment can be extreme, and under the flight circumstances expected, could easily snap the wings without the proper structure. Another downside to these conventional airframes is the landing conditions required. While some foam sUAS aircraft are hand-launched and belly-landed, many still have landing gear and require some form of a runway for landing. This can be problematic for this project, as improved landing surfaces are not a guarantee.

A delta wing, while typically less achieving in the aerodynamics category due to lower aspect ratios (EQs 1-4), does have several strong benefits. The biggest advantage a delta wing has 
is structure factor. By design, these aircraft have minimal weight that isn't immediately used. Unlike a conventional airframe, where structure weight to ensure the wings don't buckle midflight is a major consideration, the entire airframe acts as the wing. This is why some delta wings are dubbed "flying wings". The same weight required for the fuselage to maintain shape to hold avionics and cargo mid-flight is the same weight required for the wings to withstand aerodynamic loads. This leads to much lower structure factors, allowing for more weight capacity allotted for payloads, avionics, and other on-board systems. Delta wings also gain a benefit in high angles of attack, which are most common during takeoff and landing. Depending on the wing's planform shape, at angles of attack that would normally stall conventional aircraft, a delta wing can actually maintain flight by utilizing lift vortices generated by the nose and leading edge of the aircraft.

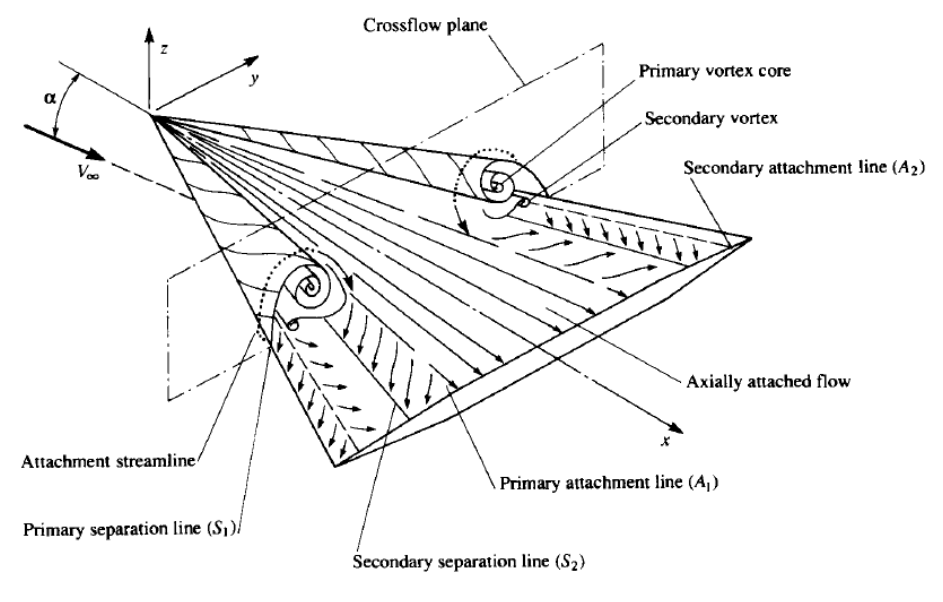

Figure 13: Example of vortex lift on general delta wing planform ${ }^{32}$

This can allow the delta wing aircraft to maintain favorable stability and control in flight conditions that conventional airframes would typically stall in. The induced vortex driving this lifting action is viable on delta wings (especially low AR planforms) due to the increased airfoil length near the root of the wing that delta wings typically feature. This induced vortex would not be present on the slender wings of a conventional airframe. Rather, there is a large separation 
bubble where free-stream airflow is no longer attached to the wing, forcing recirculation of downstream air back up the length of the wing (severity depends on angle of attack), which results in a stall.

Another advantage of a delta wing (for this project, can be a disadvantage for other deigns and situations) is maneuverability from a reduced aspect ratio. This can be further aided by keeping all avionics and other internal components as near to the centerline as possible, which drives the aircraft's moment of inertia down even lower, allowing for rapid movements. This can be very advantageous when needing to execute rapid, precise movements, all while avoiding the increased structural cost associated with conventional airframes. Also, as delta wings are naturally stable in pitch, a traditional horizontal tail surface is not needed, further reducing both complexity of the design and weight required for control of the aircraft.

Despite these benefits, delta wings still come with a few major drawbacks, the most notable in aerodynamic performance. Due to large wing surface areas, there is more skin friction drag for the same amount of lift than for a typical conventional airframe. This usually means that delta wings are intrinsically not nearly as efficient for long range or high endurance flights as conventional airframes are. Another drawback, depending on the design, is directional stability and control. Some delta wings maintain a traditional vertical tail, while some rely on winglets. While each have their own merits while accomplishing the same goal, the lack of a traditional fuselage is the primary cause of this issue. To circumvent this, the aircraft either needs a fuselagetype shape in the center of the wing or large vertical stabilizers. Some military delta wings circumvent this by using exhaust ventilation and/or thrust vectoring to give the same stability and control without requiring the vertical structure. Though this is usually done to reduce the frontal radar cross section (RCS), this method could also be used to reduce the frontal surface area of the aircraft, which would be a major benefit when considering the flight environment expected in this 
project. Another downside to delta wing aircraft is difficulty in balancing the center of gravity. Due to the aircraft's planform generally being triangular in shape, a majority of the structural weight of the aircraft is in the aft end. This requires a significant portion of the internal components being shifted towards the nose to balance the aircraft out. Should the aircraft not be properly balanced when manufactured, instead of a simple shift in weight as a conventional airframe would have, delta wings typically require ballast to be added instead. This is prevented by having a well-designed aircraft.

With the general strengths and weaknesses of each planform type described, it comes as no surprise that for the purposes of this project, a delta wing airframe was chosen as the primary category. This was due mostly to two factors: reduced structure factor and low aspect ratio. The option of reducing frontal area (which can reduce the chances of debris impact) was desirable, as were the additional takeoff and landing options available from high angle-of-attack vortex-liftgenerating maneuvers.

\section{$\underline{\text { 3.2.2 Propulsive Considerations }}$}

After planform type, the next major consideration is how the aircraft will be propelled through the air. There are again two major categories, each with two subcategories: external vs internal propulsion.

Many sUAS are operated using motors with propellers mounted on them, which is being referred to here as an external propulsion source type. Typically, foam airframes are operated using all electric motors with plastic or carbon fiber propellers, while composite sUAS that feature propellers tend towards gas-powered motors with wooden or carbon fiber propellers. The electric motors are appealing options, as there is no change in CG over the flight time due to 
static batteries, allowing for batteries to even act as a ballast in some cases. Electric motors also typically don't generate noticeable vibrations during operation. However, a major drawback is endurance. Electric batteries are not efficient energy storage solutions, and for long range or endurance flight typically require massive batteries, as do batteries for aircraft requiring high speed due to the heavy amp draw from the motors. To help circumvent some of these issues, there are also gasoline-powered motors that can drive propellers as well. This is generally present on heavier aircraft, as the power draw required for electric motors to keep a heavy composite airframe in the air is high enough that large capacity batteries would be needed. Gasoline has a much higher energy density than batteries do, and as gas burns, overall aircraft weight decreases, which reduces the thrust required to maintain a given flight condition. This allows for longer endurance flights using gasoline as compared to the equivalent weight in batteries would provide. The downside to using a gasoline motor over the electric motors is vibrations generated from the pistons during operation. Since the motor must be secured to a very rigid structure, all the vibrations generated from the pistons actuating on the motor are passed on to the airframe. This can be circumvented by applying vibration dampening surfaces to any on-board sensors, but this is still a concern that warrants consideration. In general, regardless of whether using gas or electric motors, using propellers are more propulsively efficient than using an electric ducted fan or other form of turbines. This does not come without a shortcoming of its own, as using a propeller of any form requires the prop to be in ambient airflow. This can prove problematic when there is a chance for debris or hail to strike the prop, potentially causing the propeller to break mid-flight. As there is a high chance for one or both of these to be present in the operating environment, a propeller was not greatly considered.

The next propulsive category relevant for this size of UAS are electric ducted fans (EDFs) or turbines. Having these two lumped into the same category might be read as if an EDF is simply an all-electric version of a diesel-powered turbine, though that is not the case. They are 
lumped together due to the overall similarities in integrating each of them into an aircraft. Many air-breathing turbines used by sUAS are operated using centrifugal compressors rather than axial compressors. A commercial airliner's turbine is operated using an axial flow compressor, which requires the intake airstream to be clean to function properly, where "clean" refers not only for the inlet air approaching the compressor to be perpendicular to its face, but for it to be doing so downstream of the inlet of the turbine as well. This is one reason why commercial airliners mount their engines on pylons on the wings is to promote that clean intake airflow. EDFs run on much the same premise: they need clean airflow from the ambient air to the fan disc face. This means that if an EDF is mounted internally (as would be preferred for this project), there would need be a large hole on the front of the aircraft that reaches back to where the EDF sits. Not only would a large hole need to be cut, but there would also need to be an inlet stream tube placed along the length of the hole to keep the airflow as clean as possible as the air passes from the entrance of the hole to the fan disk face. This adds unnecessary weight to the nose, unnecessarily complicates the design, and still exposes the fan blades to the ambient environment. Using an EDF is also difficult for a project such as this due to battery life as well. The same struggles outlined for allelectric motors in the previous paragraph still hold true here: the weight in batteries required to maintain high speeds or long endurances are much greater than would be required from a liquid fuel source. As such, EDFs were dropped from consideration.

The turbines commonly used in sUAS use centrifugal compressors, which operates similar to a vacuum cleaner's motor. The intake airflow is forced to the outer edges of the compressor disc before going through the turbine, which changes the inlet requirement from needing clean ambient airflow to simply needing a required airflow rate through the compressor disc for a given efficiency. This allows for very creative design techniques, as the turbine can be installed inside an enclosed airframe with intake holes placed in positions along the airframe such that sufficient airflow required by turbine is provided. Utilizing these unique characteristics, an 
airframe using these turbines can have very atypical intake designs applied, which can include options to completely shield the turbine from the environment while still providing the necessary intake airflow.

The primary drawback to using a turbine for this project, however, is there is currently no data on how a sUAS turbine performs when operating in an actively precipitating environment. Turbines used for manned aircraft undergo extensive testing with water injection tests as well as FOD injection to ensure the turbines can operate in a wide array of environmental circumstances. There is currently no data on sUAS turbines being tested for any off-design considerations, which correlates to a certain amount of uncertainty in the performance of a turbine as it is subjected to any imbalances, including but not limited to water ingestion. Being one of the larger unknowns in the design, many considerations must be taken to minimize the likelihood of water entering the turbine. With the pros and cons of each propulsive type listed, the diesel-powered turbine option offered the highest chance of reliability coupled with the best design integration options, so it was chosen to be the propulsive source for this project.

\subsection{Initial Aircraft Design}

From the previous section, the generalized design of the aircraft was shown to be reduced to a delta wing aircraft with a diesel-powered turbine for operation. In order to satisfy the design requirements, the aircraft would also need to be capable of launching and landing with no runway, maintain a cruise speed of $100+\mathrm{mph}$, top speed of $140 \mathrm{mph}$, minimum speed of at least $60 \mathrm{mph}$, have sufficient endurance for data acquisition ( 15-20 minutes), and be capable of operating in any environment. 
This section outlines the origins of the aircraft chosen to be the Tornado Intercept Aircraft for Measurement of Atmospheric Traits (TIAMAT, TIA for short). Not all of the data tested and results gathered from the originating aircraft designs are presented, but the parameters pertinent to the design and implementation of such a severe weather aircraft are explored with supporting test data to show the viability of this airframe.

\subsubsection{Diamondback Airframe}

The initial design for Diamondback came from a Senior Capstone project at Oklahoma State that takes the form of an annual competition: Speedfest. The original design was born from Speedfest VII (2017) to be a fast-moving (100+ knot), light weight (under 7.5 pounds GTOW), jet-powered aircraft with a small ground operation radius (less than $15 \mathrm{ft}$ ) that was intended to have as low of a frontal RCS as possible using only publicly available information. Speedfest VII's requirements and objectives are listed below:

\begin{tabular}{|c|c|c|}
\hline Speedfest VII (2017) & Threshold & Objective \\
\hline \begin{tabular}{|c} 
Ease of Operation (Time from \\
unbox to flight ready)
\end{tabular} & $20 \mathrm{~min}$ & $10 \mathrm{~min}$ \\
\hline Ground Operation Radius & $100 \mathrm{ft}$ & Best of Competitors \\
\hline GTOW & 9 & 7.5 \\
\hline Endurance & 3 min@WOT & 5 min@WOT \\
\hline Max level flight speed & $120 \mathrm{kts}$ & $\begin{array}{l}\text { Best of Competitors (up to } \\
173 \mathrm{kts} \text { ) }\end{array}$ \\
\hline Evasive Manuevering & $\begin{array}{c}\text { Horizontal figure 8, Cuban 8, } \\
\text { Immelmann turn }\end{array}$ & Best of Show \\
\hline Unit Cost Bid & $10,000 /$ plane & Lowest certified bidder \\
\hline $\begin{array}{l}\text { RCS (open source design, } \\
\text { justify to judges) }\end{array}$ & - & Best judge score \\
\hline Reliability & $\begin{array}{c}2 \text { consecutive flights with } \\
\text { single plane in } 10 \text { minute } \\
\text { window }\end{array}$ & $\begin{array}{c}\text { most consectuive flights with } \\
\text { single airframe over entire } \\
\text { event }\end{array}$ \\
\hline
\end{tabular}

Design Requirements/Constraints Utilize Kingtech K45 turbine FOD resistance for turbine Aircraft and all ground support equipment fit inside $48^{\prime \prime} \times 84^{\prime \prime} \times 27^{\prime \prime}$ outer dimensions Provisions for FPV system Sufficent avionics bay for autopilot system GTOW under $7.5 \mathrm{lbs}$ Good handling quallities Minimal pitch moment change with thrust over entire envelope

Table 3: Speedfest VII design constraints and objectives ${ }^{33}$ 
The RCS requirement drove the design to a delta wing planform shape with an internally buried turbine. There were several different wing configurations that were tested. Each planform type is described below in the order they were tested.

- Traditional Delta (Figure 14, top left): Flown with a $60^{\circ}$ delta configuration. Flew stable in all aspects except for high alpha flight, where there was a noticeable amount of wing rock. Became the baseline flight to compare all other flights to. Easy to manufacture with low RCS due to only having two leading edge reflection angles, but did encounter wing rock, required CG was too far forward, and wasn't a unique design solution.

- Double Delta/Gothic (Figure 14, top middle): Grouped together since they are very similar. Had an easier time managing the CG with the required range being more rearward than the traditional delta, but was not stable at high angles of attack. The wing rock was so severe it actually caused the aircraft to roll over completely, from right side up a full $360^{\circ}$ back to being right side up. Immediately rejected because of the premise of high alpha instability. Also was not chosen because of the multiple angles seen from the front, having all radar reflected in as few directions as possible decreases the effective RCS of the aircraft, and the double delta configuration gives 4 directions from the leading edge alone.

- Clipped Diamond (Figure 14, top right): Flew with a $60^{\circ}$ delta configuration that was clipped at the back at an angle of $15^{\circ}$. Gave superior RCS capabilities due to having no traveling wave reflection from the trailing edge, but required CG location was too far forward to be easily managed and had severe wing rock at high angles of attack.

- Swept Delta (Figure 14, bottom left): $60^{\circ}$ delta configuration with wing layout like that of the Clipped Diamond, but instead of removing area, adding to it. This increased wing 
area on the rear section of the craft allowed for easier cg management (pulled it rearward) and brought the aerodynamic center backwards as well. Proved to be the most stable at high AoA. Even had the vertical tail fall off in a high alpha maneuver and had very miniscule wing rock. Maintains favorable RCS characteristics like that of the Clipped Diamond while negating all the unfavorable associated with the removal of wing area.

- Variant of Boeing Bird of Prey (BoP) (Figure 14, bottom middle): $60^{\circ}$ sweep delta configuration that had raised outboard sections of the wing. They acted like winglets, but instead of being purely vertical, they extended up and out from the wing at a $30^{\circ}$ angle. This design was the first that alerted the team to alternatives to a full tail. The BoP had favorable high alpha characteristics that we believe were due to the extra vertical area on the outboard section of the wing that would be caught in the vortex flow coming off the nose when in high alpha flight. The downside to this design was a difficult to manage CG (had flat trailing edge) and was difficult to build. However, it gave the team a unique idea, leading too...

- Modified Swept Delta (Figure 14, bottom right): This was the chosen design and was based heavily on the original swept delta with the addition of knowledge gained from the BoP design testing. It was a swept delta with winglets on the end and no vertical tail. It had no wing rock when at high AoA, could spot land in under 15 feet, had favorable RCS characteristics, was very maneuverable in the air, had a very favorable rear CG location, left the most space of all designs for incorporating space into the vehicle, and was very easy to manufacture. This was the design chosen to go forward with a composite build. 

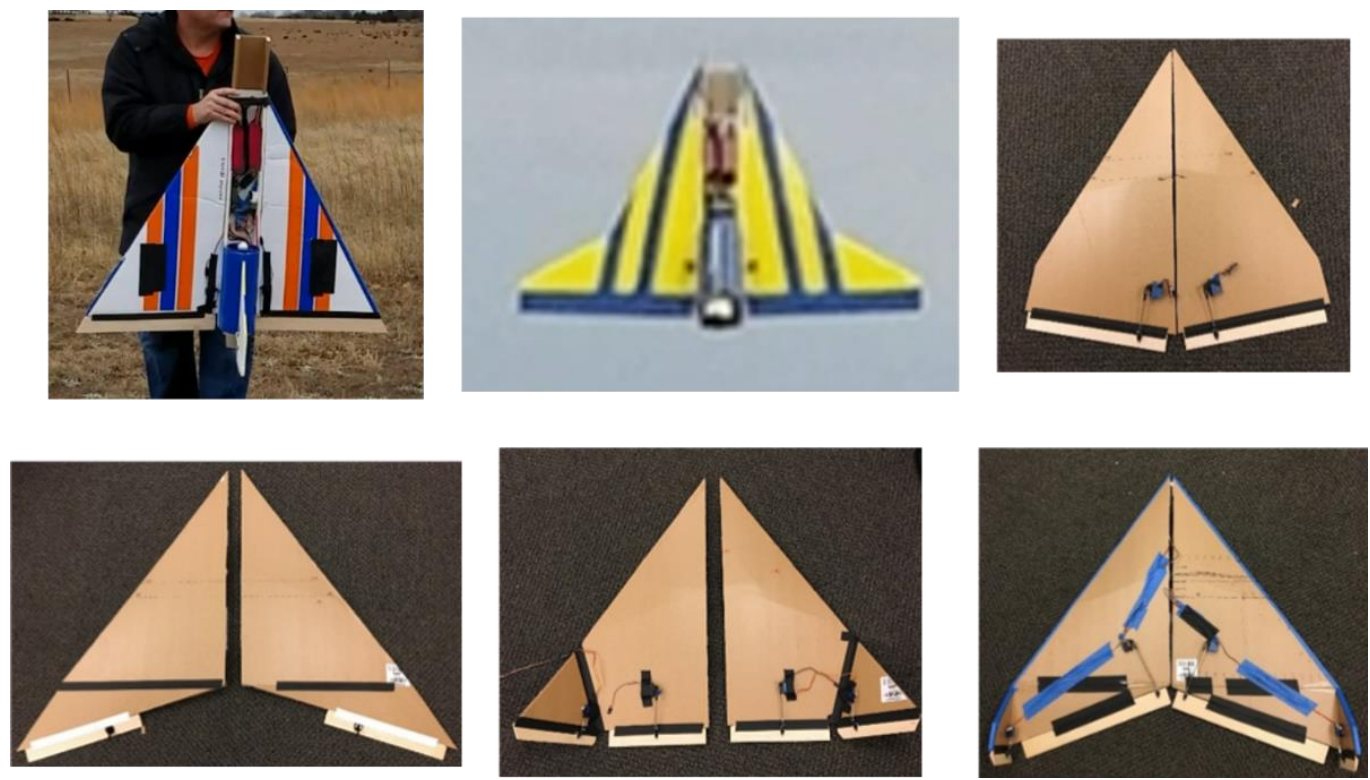

Figure 14: Flight tested planform types

Of these different wing planforms, the Modified Swept Delta performed the best. It had the lowest amount of wing rock at high angles of attack to support the required spot landing capabilities and maintained favorable pilot maneuverability in all aspects of flight more consistently than any of the other options tested. All iterations were flown with the same pilot for consistency in handling qualities.

With the wing condition established, it's important to note how vital the winglets are to the aircraft as a whole. Without them, the aircraft would not be nearly as stable at high angles of attack and would be more susceptible to not only wing rock but also have reduced directional stability. A traditional tail mounted at the rear of the fuselage in the center directly over the thrust vectoring would be able to help in all areas of this with the exception of high alpha flight.

Repeated tests have shown the winglets, when placed on the outboard section of the wings, are directly in the vortex wake from the nose and leading edge of the aircraft, and being in the middle of this high energy flow is why the aircraft maintains stability beyond the normal flight regime as 
angle of attack is increased. In addition to this, a thrust vector system is used to give direct yaw control and aid in high alpha maneuvers.

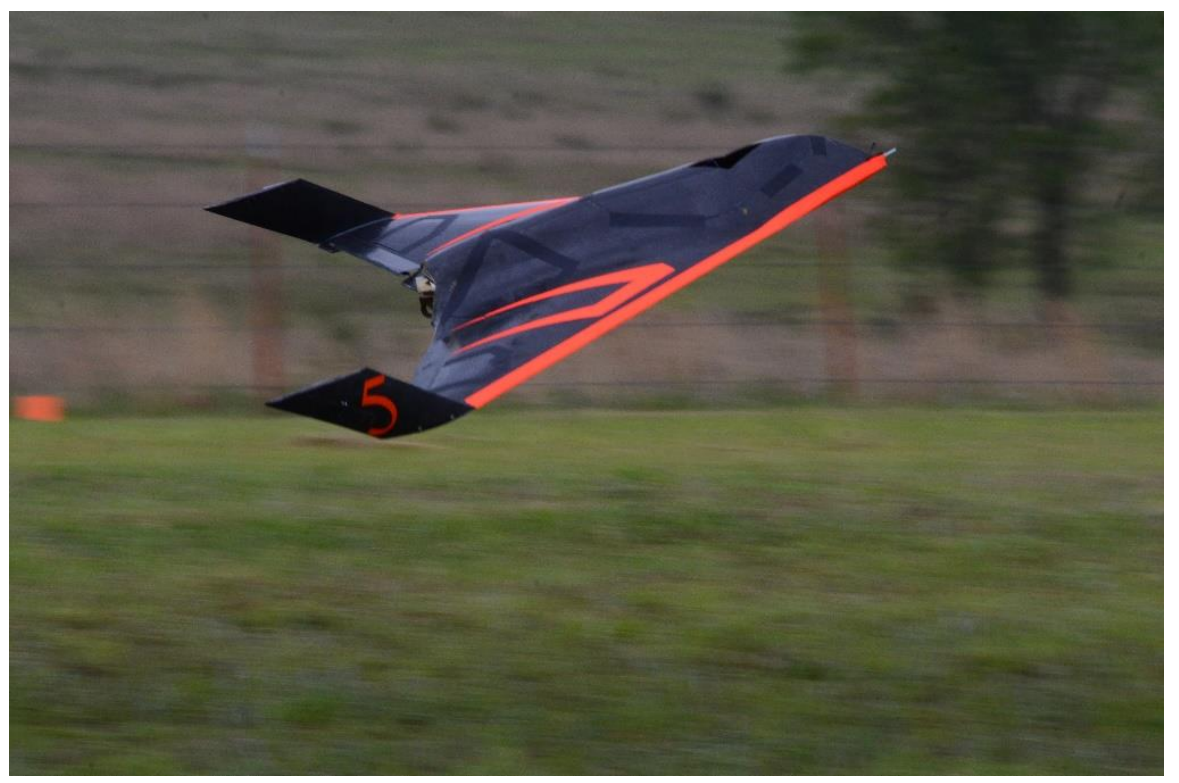

Figure 15: Diamondback in landing approach at high angle of attack

\subsubsection{Internal Layout}

With the wing configuration established, interior layout became a primary factor. Needed to be installed were a Kingtech K45 turbine and its required components, Futaba RC receiver, compatible airspeed sensor, fuel tanks to support a minimum of 5 minutes wide open throttle, and a rate gyro to assist in takeoff and landing with high alpha maneuvering. Since the propulsion system as a whole weighed $67 \%$ of the total aircraft weight, its location was the most vital. Given the relative disposition of delta wing aircraft, the turbine was forced to be placed in the nose of the craft to properly balance the center of gravity (CG). This left great concern of what to do with the intake for the turbine in terms of the UAV's RCS capabilities. One thought was to use some form of a mesh in the very nose of the aircraft to allow for air to pass through while still reflecting 
the EM waves away, protecting the turbine from reflecting radar waves from the turbine's compressor blades back towards the target. Since the design was already borderline on weight allotment, this was not well received due to the extra weight that would need to be added for an aluminum mesh. Another alternative was a scoop made of fiberglass placed on top of the craft. This was favorable because of the light-weight nature and the fact that fiberglass is mostly radar transparent, but it was concluded that with the severe curvature of the nose where the scoops would need to be located would be a hindrance to the RCS characteristic due to the removal of the radar reflective carbon fiber skin to allow for the inlet scoop. The chosen design was a diamond shaped hole cut out on top of the craft with the center placed forward of the turbine's intake to allow for full airflow without any loss in RCS capability. This was verified through testing on a thrust cell to have less than 5\% losses. Propulsive testing of the prototype can be found in Appendix B, as well as testing for a pure reverse-flow intake that showed promising results.

To be noted is the effect a bell mouth has when placed on the backside of an operating turbine. The required intake area of the K45 engine when running with no jet pipe or bell mouth is approximately $2.5-3 \mathrm{in}^{2}$. When a jet pipe with a bell mouth attached are installed behind the turbine, that required area increases to nearly $12 \mathrm{in}^{2}$ to achieve the same level of losses. The bell mouth also determined the maximum diameter of the fuselage section of the aircraft. The bell mouth has a diameter of 5 inches, so the craft was determined to have a diameter of 6 inches to ensure no part of the airframe would melt or combust due to the extreme heat exhaust gas of the turbine $(650 \mathrm{~F})$. 


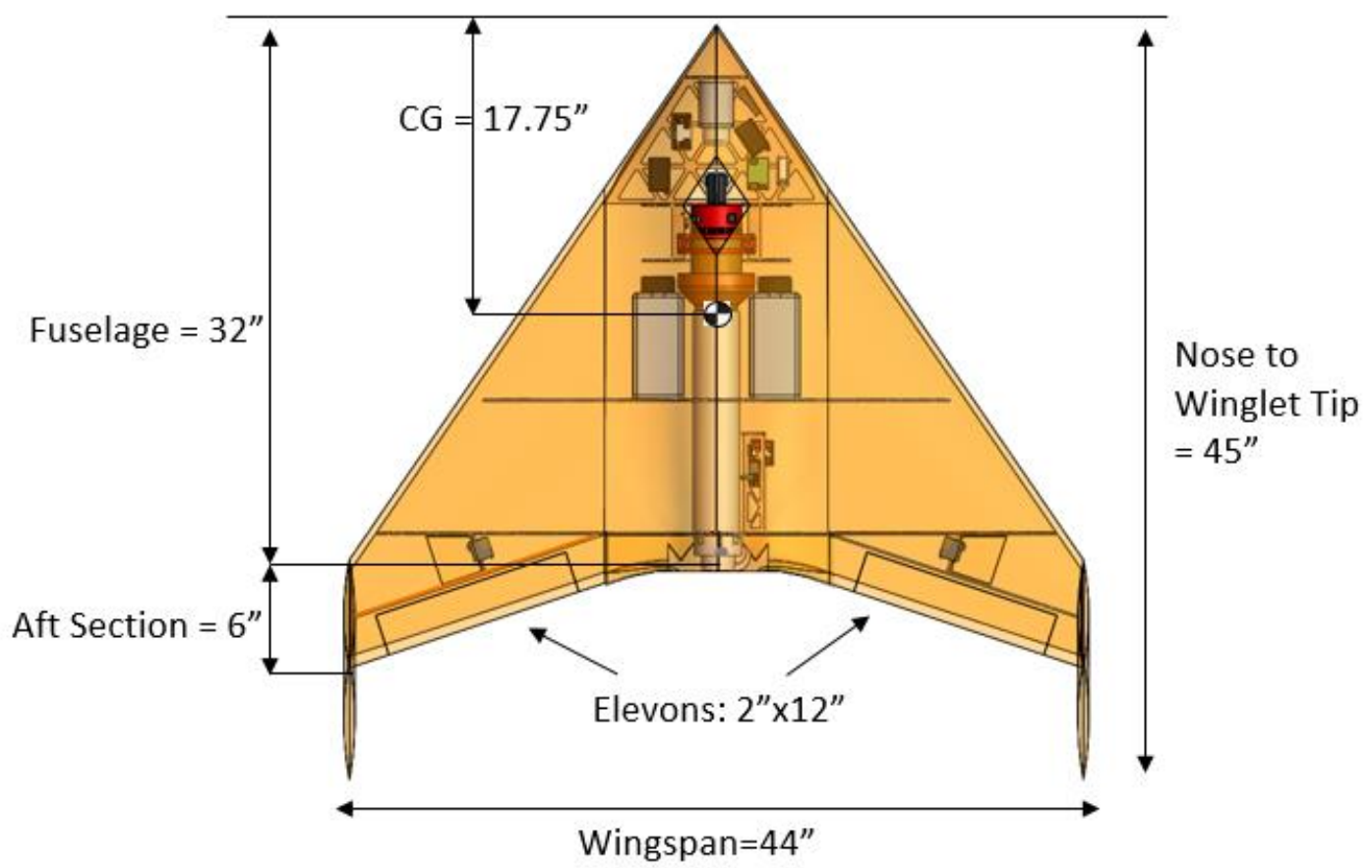

Figure 16: Dimensions of Diamondback

As for area inside the wings, the airfoil geometry was chosen to be a biconvex airfoil with a maximum thickness of $10 \%$. The biconvex was chosen not only for RCS purposes, but works well at high AoA and would be easier to manufacture than a NACA series airfoil. The $10 \%$ thickness was chosen based on drag performance characteristics of a biconvex airfoil. A plot was established relating the coefficient of drag to the different max thicknesses of biconvex airfoils, and the $10 \%$ metric allows for the most internal room with minimal additional drag, as can be seen in Figure 17. Even the winglets were chosen to be biconvex for similar reasons, as they still had available internal volume to hold servos in the winglets for additional yaw control should they be needed. 


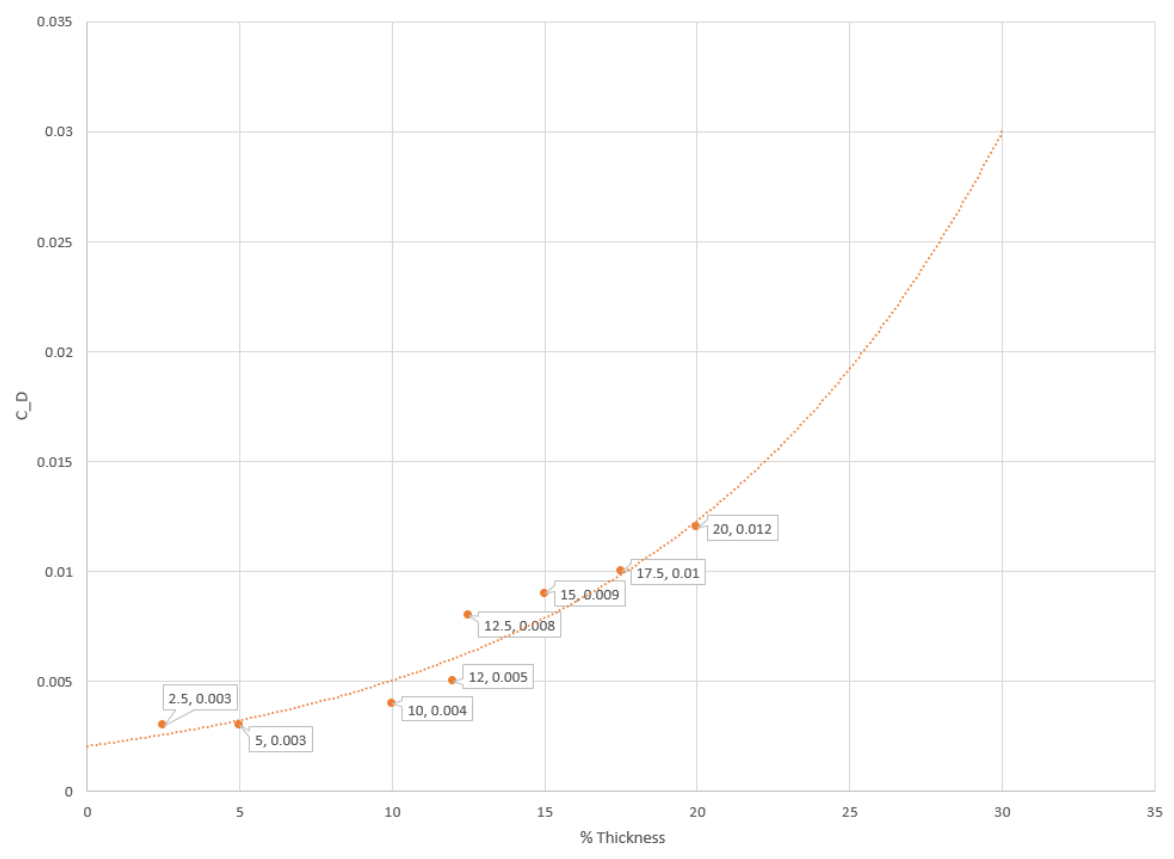

Figure 17: Coefficient of drag of biconvex airfoils as a function of max thickness

The actual CG balancing proved to be fairly simple once the components were all finalized. Airspeed sensor, Futaba receiver, batteries, and all the engine components were placed in the nose to minimize wiring length. These were all placed on a designated tray to elevate the electronics off the base of the aircraft. This was incorporated in the event fuel leaked mid-flight and the fuel happened to reach the nose during a maneuver, the avionics would have a greater chance of survival. Aft of this tray the turbine was mounted on its own structure to prevent it from twisting off the airframe, and between the turbine and its mount was rubber pads to dampen the impulse generated from belly landing the aircraft.

The jet pipe stretched from the back end of the turbine out the back of the aircraft with the throat of the bell mouth placed 0.5 inches from the nozzle of the turbine. Aft of the bell mouth on either side of the jet pipe are $24 \mathrm{oz}$ fuel tanks. This placement was tested and proven to be low enough in temperature to allow the plastic tanks to not melt in flight, yet also be placed on the CG to minimize the CG travel as fuel is burned in flight. 
After the required avionics were determined, the fuel locations were finalized. To help maintain proper balance, three total fuel tanks were used: one 24 fluid ounce tank on each side of the jet pipe and one 5 fluid ounce header tank in the very nose of the aircraft. The header tank was placed in the nose primarily as an after effect of the rest of the component install, as it ended up being very nose heavy. The two rear tanks allow for max fuel volume capability using commercially available fuel tanks, and the header tank acts as a reservoir for the turbine to draw from directly. The turbine draws fuel directly from the header tank via the fuel pump, and from the header tank the fuel line T's off to each of the rear tanks. This allows for even draw of the fuel tanks, and the header tank prevents air bubbles from reaching the turbine and killing it mid-flight. The header tank was also placed in the nose of the aircraft to counter-balance the aft-heavy nature of the aircraft.

In the rear are 4 servos, one for each elevon and one for each control movement of the thrust vectoring unit. This initial design also had the capability of adding servos in the winglets for additional yaw control, though that avenue was not actively pursued. The elevon servos were attached in the middle of the control surface for increased rigidity of the entire surface. The Aura 8 rate gyro was placed in the back of the aircraft to reduce wiring demands of the entire system. A single wire connects the Futaba receiver to the Aura 8 via SBUS, and the Aura 8 takes that input and splits it into the corresponding roll, yaw, and pitch commands that are sent to each servo. The Aura 8 was programmed to mix signals in such a way that the thrust vectoring responds to pitch and yaw commands just as the elevons respond to pitch and roll commands.

The primary benefit of using the Aura 8, other than for convenience of signal mixing, is for variable rates and gains for the different expected flight modes. The aircraft had one set of 
tuned rates and gains for hand-launched takeoff, another set for cruise, and a third set for high speed flight.

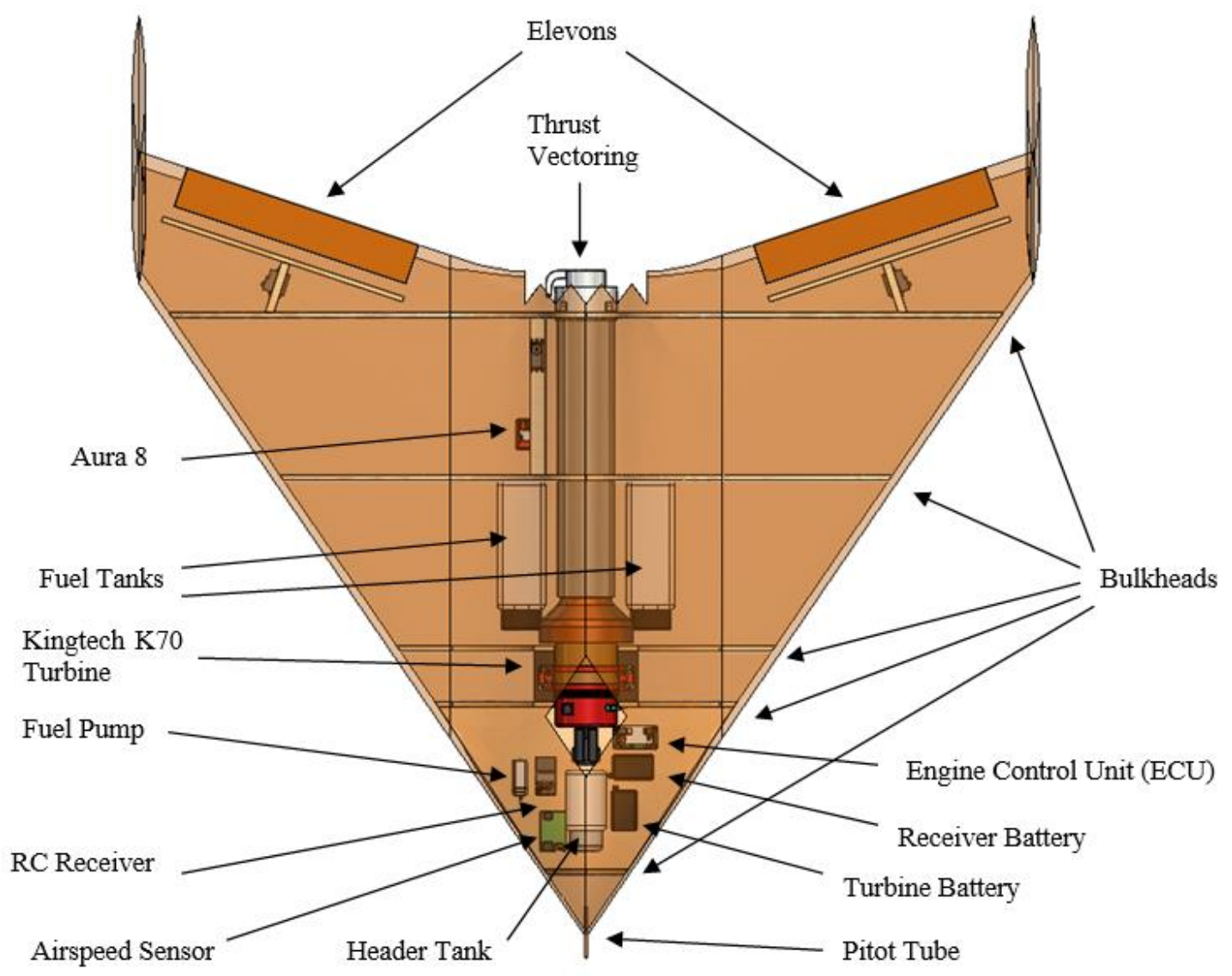

Figure 18: Layout of initial version of Diamondback

This layout resulted in an initially estimated SM of $12 \%$, which was predicted to be sufficiently stable in all aspects of flight since there would also be an on-board rate gyro. When the actual aircraft was developed and flight tested, SM was actually closer to $7 \%$ yet remained stable through all flight testing that the airframe was subjected to.

\subsubsection{Diamondback Performance}


This configuration of Diamondback served its purpose well, being able to fly for $\sim 6$ minutes with a wide open throttle and $\sim 10$ minutes at half throttle. It clocked a max speed of 142 knots ground speed, had a radius of operation less than 20 feet, was rapidly deployable via hand launch, and easily recoverable with a high angle of attack belly land. The turbine inlet style and location minimizes the fear of water ingestion as the liquid cannot make the $90^{\circ}$ bend downward during flight into the turbine inlet. It is instead sucked into the jet pipe, reducing the chance for the water to put out the combustor's flame.

However, when determining a suitable aircraft for severe weather applications, a much longer flight time is needed to allow for time to travel to a target, get the data, and return to launch safely. It will also need to be equipped with an autopilot and weather sensing instrumentation to fulfill its purpose as an all-purpose severe weather UAS. Though the initial version of Diamondback has satisfied many requirements, it was partially redesigned to achieve the range, endurance, and sensor requirements.

Though much effort is shown in future sections of how the aircraft was made to best suit the task at hand, there are some inefficiencies that exist due to the repurposing of the airframe. In particular is the airfoil: a biconvex airfoil is not as efficient at producing lift as a cambered airfoil, which results in a higher throttle setting required to maintain a given speed. This reduces the top speed achievable by the aircraft, as well as reduces the max range and endurance the aircraft can achieve. However, a cambered airfoil also factors in a larger aerodynamic moment, which could potentially be more difficult for the tailless delta wing to counterbalance. All these factors would need to be considered before addressing what a more ideal airfoil for this aircraft would look like, which is beyond the scope of this project. Acknowledgement that not all design parameters of the aircraft are ideal is necessary, for even though the redesign allows the aircraft to accomplish the 
target objectives, the same aircraft could perform the mission more efficiently with a few design modifications, namely airfoil and leading edge sweep.

\section{$\underline{\text { 3.3.4 Comparison of Diamondback to Other Aircraft }}$}

As shown in Section 2.2, the majority of severe weather research aircraft are conventional aircraft with long wingspans, high aspect ratios, and prop-driven propulsive systems. These systems each have their merit as outlined in Sections 3.2.1 and 3.2.2, which are primarily the long endurance and range each can achieve. Simply having a long range or endurance does not have any bearing on the performance inside a severe system, in particular when approaching an area with extremely high winds $(75+\mathrm{mph})$ that are variable both laterally and vertically. These aircraft must have an increased structure factor to withstand these variable asymmetric loads or simply avoid these conditions entirely. Assuming the aircraft are built to withstand all these conditions, the operational requirements are not as convenient as a delta wing such is proposed here: a low aspect ratio delta wing has a larger airspeed range (lower stall speed, higher top speed) and is more maneuverable (ie controllable in larger asymmetric wind loading

conditions) than a high aspect ratio conventional wing. These qualities coupled with the turbojet propulsive system ensure the aircraft will be capable of much higher top speeds than the propeller driven aircraft at similar takeoff weights.

The primary drawback to a low delta wing aircraft is the reduced range and endurance, as those categories are where conventional aircraft excel. These drawbacks are more than tolerable, as the purpose of the redesign of the aircraft is to allow the plane to launch from a remote location, rapidly approach a target phenomenon, loiter and gather data for up to 10 minutes in a very turbulent, gusty environment, then return to the operators and land with no runway. Most 
other aircraft are designed for long-term monitoring of a moving system, which is not the objective of this aircraft. This aircraft is a targeted observation aircraft as opposed to the monitoring aircraft typically seen.

\subsection{Structural/Internal Redesign}

This section includes the process of redesigning Diamondback for much longer flight durations, increasing from the 6 minute wide open throttle endurance to 14 minutes, and from an 11 minute cruise to 25 minute cruise. It also includes the process of integrating a Pixhawk 2 autopilot on board and the use of two rate gyros (Pixhawk and Aura 8) in series to give differing levels of controllability for different flight regimes. This process has been completed and the aircraft is fully integrated with all avionics systems that were included in the redesign (autopilot, video system, long range RC and telemetry link). Other new features include: Kevlar lining down the middle of the aircraft on the bottom skin to aid in puncture resistance on landing, custom form-fitted fuel tanks that increased total fuel volume from 50 fluid ounces to 120 fluid ounces, and a redesigned internal layout to accompany all the new avionic system components.

\subsubsection{Additional Equipment}

The primary drawbacks that prevent a direct use of Diamondback are: low flight time (endurance/range), lack of telemetry systems, no failsafe modes, and there are no provisions for sensor mounting. With all this extra weight added, the Kingtech K45 turbine that was used in the previous version will not provide sufficient thrust for flight operation, specifically takeoff. For this reason, the turbine was upgraded to a Kingtech K70, which is a redesigned K45 that uses the same housing but uses a better compressor and a higher fuel flow rate. This requires some minor 
internal modifications to achieve the target operational characteristics. Namely, custom fuel tanks will need to be fabricated to fit in the fuselage to dramatically increase flight time to the target of double the current flight time. A Pixhawk 2 flight controller will need to be installed for autonomous capabilities and for remote monitoring as the aircraft approaches a severe weather phenomenon. This means not only the Pixhawk board must be installed, but also a HERE 2 GPS puck, a new airspeed sensor, and an RFD900x telemetry module. For situational awareness as the aircraft approaches the target, a first person view (FPV) system must be installed, which includes a camera, video transmitter (VTX), and antenna. The VTX tends to get very hot when operating, and as such typically features a heat sink with access to clean airflow for cooling. Due to the long range flights that will ensue, a DragonLink V3 system will need to be installed for long range RC control of the aircraft. The ways each additional component will be installed is outlined in the following subsections.

\subsubsection{Custom Fuel Tanks}

Though a new engine was designed around, since it uses the same housing, no modifications were made for it. However, this turbine burns fuel at a higher rate $(155 \mathrm{grams} / \mathrm{min}$ vs 230 grams/min), giving even more importance to the increased fuel requirement. To combat the low flight time, new fuel tanks were designed with the intent to increase the total fuel volume from 48 fluid ounces to a goal of over 100 fluid ounces. The design constraints of these tanks were: form fit the inside of the fuselage to hug the interior wall, be symmetric where one mold can make a tank for either side (reduces manufacturing costs), use 0.5 " rounded corners on all edges (manufacturer's requirement for Kevlar composite layups), and hold a minimum of double the fuel volume. 
The design began in CAD (SolidWorks) by implementing an offset of 0.25 " from the inside skin. The smaller portion was used as the outline for both halves of the tank as it must be symmetric for both tanks to use the same mold. This went through several iterations keeping the internal structure the same, but no design could garner more than 75 total fluid ounces. This led to a partial structural redesign. The airframe as a whole is overbuilt structurally, which is a primary benefit to using this airframe, but also includes some unnecessary weight. One location in particular was the mounting bracket for the thrust vectoring servos. They used an L-bracket to support the servo tabs, but two inches forward of this bracket is the main bulkhead. If the bulkhead were moved back to the same location as the L-bracket, the fuel tanks would be capable of holding 120 fluid ounces, which translates to a flight time of 25 minutes at cruise (50\% throttle, 100 knots) and 13 minutes at max throttle (140 knots). This bulkhead movement was deemed appropriate as a single airframe had undergone 40+ flights without any structural damage. The main bulkhead was then modified (in the CAD) to possess the same features the Lbracket had, effectively eliminating the need for that portion of 5-layer plywood. This extra space allowed for the tanks to surpass the goal of doubling the total fuel volume while still being centered on the CG. The CAD was then converted to CNC file, of which a foam plug was machined to the exact specifications. The plug was then prepped fully to be the negative for a mold. The plug was then shipped to Jet Tech USA for manufacturing of the mold and all future fuel tanks. For puncture resistance and overall strength-to-weight of the tanks, the tanks were made using two layers of Kevlar with water-tight epoxy.

\subsubsection{Long Range Autopilot Communication}

With the fuel tanks redesigned to increase the max range and endurance, the next step was to allow for communication both to and from the aircraft as it travels beyond visual 
situational awareness. The Pixhawk 2.1 allows for GPS tracking of the aircraft, autonomous waypoint missions (uploaded before or during flight), and changeable safe modes for various worst-case situations, all of which are required for the intended mission. The most useful is the aircraft state monitoring, as the Mission Planner console gives a full visual view of the aircraft's condition.

The Pixhawk was placed in the rear of the jet to reduce the length of wires required. A dedicated mount was fashioned out of 5-layer ply to give the Pixhawk a level platform to sit on. The mount was made to also include the Aura 8 next to the Pixhawk, as the two systems close together reduces wiring length even further. The two systems interact as follows: RC signal is sent from the receiver to the Pixhawk, where the Pixhawk treats the output as if it were controlling a standard "tube and wing" aircraft with two ailerons servos (controlled by one control signal), one elevator servo, and one rudder servo. The Pixhawk outputs these three signals to the Aura 8, where the Aura takes the three input signals and mixes them in the same way the initial version of the jet mixed them. The Aura 8 then uses four ports as outputs, one to each servo. The Aura 8 is setup to use the last port as a rate/gain switch. This allows for the pilot to utilize the Aura 8's gyro control when the Pixhawk is in manual mode, yet still allowing for the Pixhawk to output its own control while in any of the Pixhawk-based gyro control modes with the Aura 8's gyro turned off, preventing conflicting control outputs from the two systems operating simultaneously. These were implemented on the same physical switch on the RC transmitter, with Manual Mode corresponding to Aura 8 gyro on with Pixhawk gyro off, and when in any other mode the Aura 8 gyro is off with Pixhawk gyros enabled.

The Pixhawk and Aura 8 being installed nearby reduces some of the wiring constraints, but the reason for being placed in the rear of the aircraft despite the already aft-heavy design was the length of cables required to the GPS puck and telemetry module. With the Pixhawk placed 
just off-center laterally, the GPS puck was able to be installed right next to the Pixhawk. For RF interference mitigation purposes, the RFD900x was placed in the starboard winglet, which had sufficient space for mounting, with a cable running from the winglet to the Pixhawk. The RFD allows for telemetry data to be transmitted down to a ground station for monitoring, as well as receives data from the ground station as it is sent.

In order to get airspeed information, a dedicated airspeed sensor designed specifically for the Pixhawk was installed in the nose to replace the Futaba system with a data cable running aft to the Pixhawk.

For the communications system to be installed, no structural redesign was necessary, but hatch geometry did. In order to give the option for future payload delivery, the rear hatch was extended to be mirrored on both sides of the fuselage. This allows for simpler integration of Pixhawk and all wiring for the components in the aft of the aircraft. An optional design challenge was to drop a sensor package through a severe weather system, and this payload bay would allow for a single deployment of a sensor through a target phenomenon. This is not the focus of the paper, but is a consideration should time permit.

\subsubsection{FPV Video System}

Another major overhaul was the addition of an FPV video system. This allows the pilot and ground control station (GCS) operator to have visual situational awareness from the perspective of the aircraft. In order to get useful footage, the camera was installed in the nose with a slight downwards angle set. This is because the aircraft in normal flight has a very slight angle as well as to give the pilot a sense of orientation by keeping the horizon in view. 
As mentioned above, the camera is set in the nose of the aircraft and mounted to a wooden tray using screws. The hole in the nose was cut just large enough to capture the camera's full field of view. To help seal the aircraft from water ingestion around the camera, hot glue was used to fill in the voids beyond the camera's field of view, with tape applied to the camera to prevent the hot glue from sticking to the camera directly. This resulted in the lens protruding from a small cavity filled in with hot glue for sealing purposes.

The cables from the camera run aft to the VTX, which is located near the port elevon servo. It was located here to help laterally offset the Pixhawk's weight on the starboard side, as well as to keep it away from the heat generated by the turbine and the exhaust gases going through the jet pipe. Though this area is not hot enough to melt any components on its own, because the VTX gets very hot on its own, installing it in a lower temperature environment is ideal. It does not receive ambient airflow for cooling, but this is not an issue because of the relatively short flight time it will be running for. The VTX was made to operate for several hours on end (many foam fixed wing aircraft can do 1.5-2 hours of flight), and it is in these environments that airflow for cooling is required. For the shorter flight time of $\sim 20$ minutes max, the VTX will not get hot enough to cause any damage to it or the airframe.

The video stream coming from the camera goes to the VTX, at which point it is sent to the antenna in the port winglet. This is a $2.4 \mathrm{GHz}$ Vee antenna and transmits the video feed down to a Skylark screen at the GCS for the pilot and PIC to view.

\subsubsection{Long Range RC Control}

In order to have command and control (C2) of the aircraft over long distances, a long range RC system must be integrated. The standard $2.4 \mathrm{GHz}$ Futaba system, though very reliable, 
is not sufficient for flights beyond 1-2 miles. Once completed the sUAS will be required to operate up to 10 miles away, and it is for that reason that a DragonLink V3 system was chosen. RMILEC was also considered, but during testing it was determined to be more of a hassle for integration as compared to the DragonLink. RMILEC operates by receiving the Futaba (or other RC) signals, then re-transmitting them long range to the receiver on board the aircraft. This requires a separate antenna tripod for the RMILEC system, and because of the second transmission of the signal, was less responsive than the DragonLink. By comparison, the DragonLink uses a backpack that mounts on the back of the Futaba controller, which takes the direct signal output from the controller using a cable and transmitting that data to the DragonLink receiver onboard the aircraft. This means only one $\mathrm{RC}$ signal is being transmitted for the DragonLink as opposed to the two separate (one short range, one long range) that was required by the RMILEC system.

Integration of the DragonLink was also very simple, as no redesigns were necessary for it. The Futaba receiver was removed and the DragonLink was setup in the exact same way, with Channel 3 being used for throttle, Channel 1 sending the Aura 8/Pixhawk switch information, and SBUS sending all control commands to Pixhawk directly.

\subsubsection{RF Energy Management}

The primary reason for the placement of the various RF components wad not only for CG balancing, but to ensure no RF interference associated with placing different signal-receiving and transmitting devices within very close proximity. As per the design, the DragonLink RC receiver (433 MHz) was placed in the nose, the GPS puck $(1.3 \mathrm{GHz})$ placed near the middle of the

fuselage in the rear, the RFD900x (900 MHz) located in the starboard winglet, and the VTX 
antenna $(2.4 \mathrm{GHz})$ residing in the port winglet. By keeping all components operating on different frequencies and maintaining the greatest possible distance between components on the aircraft, issues relating to RF interference between components was mitigated by design.

\section{5: Launcher Design and Implementation}

When one thinks of storm chasers, it's easy to visualize a small convoy of vehicles driving out to some remote area of the countryside, using every resource at their disposal to ensure they are in just the right spot to see the phenomena spin up and to get the data they desire from it. When this concept of rapid movement to a target and uncertain terrain conditions is paired with the desire to collect data from an aircraft, one immediate challenge is faced: many aircraft require a decent runway for takeoff and landing. There are some lightweight foam fixed wings that can be hand launched, but these aircraft are not robust enough to withstand the gust loading of a tornado. As such, the focus is on designing a launcher that will be able to: deploy a heavier airframe (20+ pounds), deploy multiple airframe types (not simultaneously), require a small transport footprint, lightweight, robust, used in all weather conditions, and can account for changing wind directions (always want to launch directly into wind).

With the goals outlined, a look at what launchers currently exist was compiled. As with most cases when using a launcher, and to satisfy the landing condition mentioned above, the aircraft will be belly landing. Of the launchers analyzed, a few general categories were developed, some with more potential than others. These categories were: hand launch, bungee launch, catapult/rail launch, and roof-mounted vehicle launch. Pros and cons of each will be outlined in the following sections. 


\subsection{1: Launcher Class Options}

\subsubsection{1: Hand Launch}

The most basic way to get an aircraft into the air is to have a person physically throw the aircraft into the air. For the case of lightweight aircraft, a pilot can both launch and fly, but in most cases there is a need for a second person to throw the aircraft while the pilot controls it.

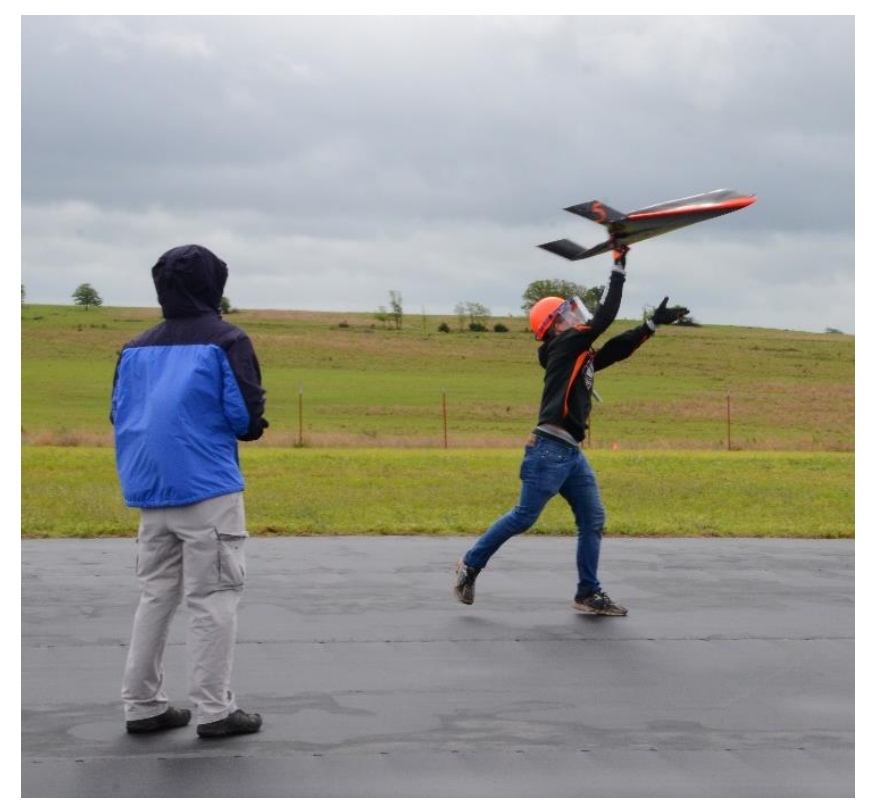

Figure 19: Pilot with additional person to hand launch

Though this is a very simply launch procedure, for the design case the benefits are

limited. There is no additional hardware required, it's very easy to change launch direction to account for winds, and the person launching can adjust how hard the aircraft is thrown to account for gusts. However, the reliability of the launch is tied exclusively to the person throwing the aircraft. This translates to having a strict personnel requirement, which is not ideal. Even with a very reliable thrower, there is still no guarantee that the launch will be successful, especially when compared to other options. These other options are explored further. 


\subsubsection{2: Bungee Launch}

The bungee launch is a very simple system consisting of a single loop of elastic rubber that is staked into the ground on one end and attached to the aircraft on the other. Generally, there is a hook on the bottom of the airframe that holds the bungee, and as the aircraft passes over the bungee stake, the bungee falls off and the aircraft is flying under its own power. Just like with hand launching, this method sometimes allows for one person to both fly and launch (lightweight foam airframes) but usually requires two people: one to fly and the other to hold the airframe to launch. This method was primarily introduced to aide in getting gliders into the air, but it has often been used to get other foam and lightweight aircraft airborne as well.

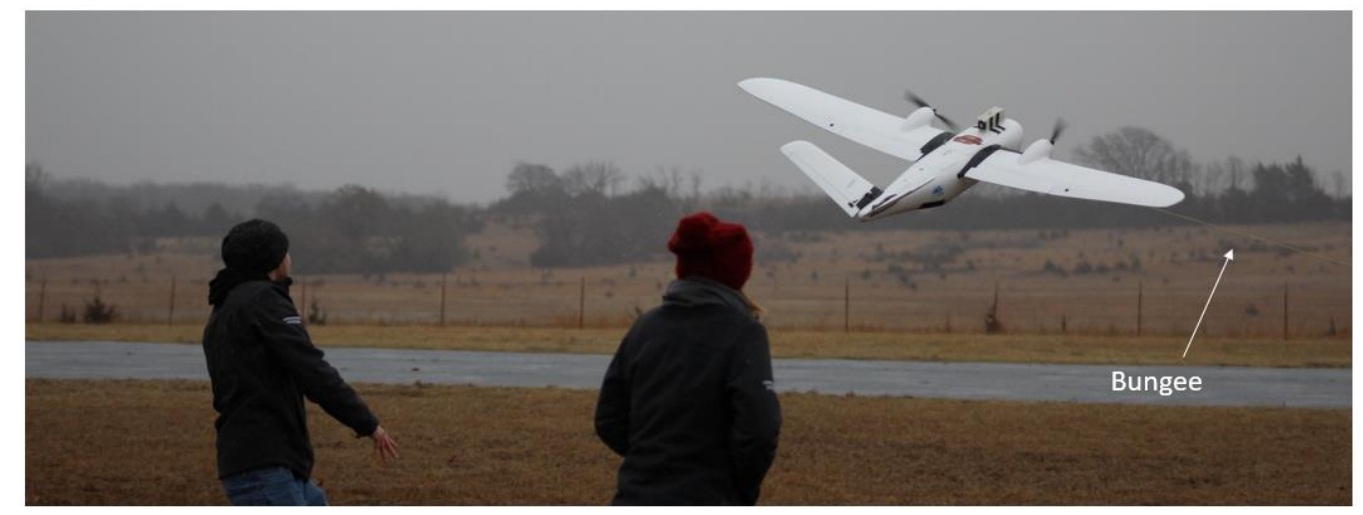

Figure 20: Bungee launch of Believer UAS

Some advantages to using this type of launcher is it is highly portable, very easy to set up and take down, is very easy to integrate to launch different types of aircraft (provided they are light enough), and very easy to change launch direction to ensure it is always into the wind. The cargo footprint is the same as carrying an extension cord and most work vehicles have a hammer or similar hard object nearby to secure the stake into the ground (stomping usually work too), making it a very versatile option. 
However, the reliability of the launch is tied to the person who does the launching. Someone who has launched multiple times will be inherently more stable on launch and know by how much the elastic needs to be stretched to ensure a successful takeoff. This increased personnel knowledge requirement, while minor, is not ideal. On the same note, it is bad for the bungee to stay loaded for more than $10-15$ seconds, as it is meant to store and rapidly release energy. Storing energy long term can cause the elastic to fail. Another setback to the bungee is the requirement of hardware mounted to the bottom of the airframe. For heavier aircraft, the protrusion on the bottom is not ideal for landing, as that induces extra undue stress on the airframe. Securing the launcher to the ground can also be problematic. Most severe systems tend to dump a lot of rain, and depending on the launch location, the ground could be very wet. This would prevent the bungee from gripping the ground very well, which would prevent a successful launch.

\subsubsection{3: Catapult/Rail Launch}

A slightly more complex style, the catapult launcher (also known as a rail launcher) uses the same basic energy storage technique as the bungee. A typical catapult launcher has a frame that the aircraft sits on, an elastic bungee stretched or wound under the frame and attached either to a sled (rail) or directly to the airframe (catapult), and uses a release pin to allow the sled/airframe to move under the influence of the bungee. The same setup can also be used for a pneumatic launch system. At the end of the launcher's frame, in the case of the rail system, the sled is either stopped or flies off the frame, allowing the aircraft to fly under its own power with nothing attached. For the catapult system, the bungee falls off the aircraft in the same way it did for the bungee launcher. When this system is used for lightweight foam aircraft, a lightweight PVC build can be used. For larger, heavier airframes, a more robust system is needed to 
withstand the loading the bungees. Figure 21 shows an example of a custom rail-launch system used to launch the TIA prototype, Beast.

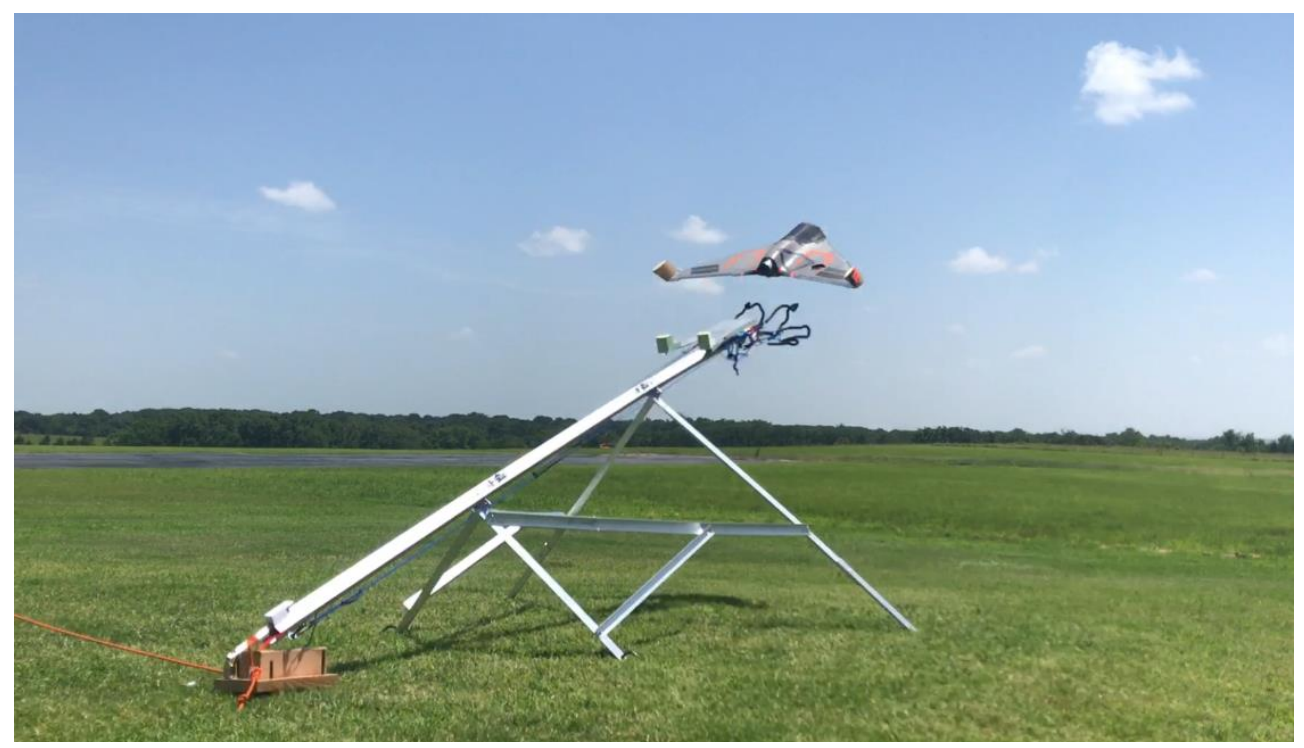

Figure 21: Catapult launch example (bungee propelled)

Though this system is very similar in energy storage as the bungee launcher and shares many advantages, this design brings about many unique disadvantages. The primary benefit of this system over a standard bungee launch is the robustness of the design, as the rail system will more reliably launch an aircraft than an operator holding and releasing the aircraft. However, this comes at the expense of many new cons: overall reduced mobility (for changing wind direction and setup/takedown), drastically increased internal cargo space requirement, and overall ease of operation. Since both system use similar types of bungees, they are still prone to fatigue failure, especially if left out in the sun for extended periods of time. As such, they require regular maintenance to ensure no cracking or other detrimental factors are occurring as well as to ensure they are exerting the expected forces. It is also more difficult to fine tune the amount of energy to be released. Aside from adding/removing bands, the launcher will launch with a fairly consistent energy. This is favorable when flying in ideal conditions, but becomes a detriment when 
considering the launcher must work both in calm and very gusty conditions. If the aircraft is launching into a high velocity head wind, it does not need to be launched with the same force as on a calm day. Like the bungee, this system also typically requires additional hardware mounted to the exterior of the airframe (catapult) or slots cut into the airframe (sled) to keep the aircraft at rest while at full throttle, but accelerate forward on launch. This makes the catapult/rail system more difficult to setup due to the addition of the frame that must be assembled when the launch location is reached compared to the bungee alone. The biggest drawback is the amount of additional space required not just for operation, but also for transport. A launcher system that must be packed away and placed in the vehicle takes up a lot more extra space than a wrapped bungee cable and a hammer, which in a field operation or storm chase event is not a viable option as space is already an issue in most cases.

\subsubsection{4: Vehicle Launch}

The final class of launching system to be analyzed is a vehicle-based launcher. There are multiples of variations that all include a roof-mounted system: some use a pneumatic or bungeebased system much like the catapult system from the previous section, others use a pin-release mechanism to launch the aircraft. In both cases, the roof-based systems can be installed prior to traveling to the launch site, meaning there is no cargo space being used in transport of the launcher. For the catapult variants, there is an angled rail the aircraft sits on and is launched identically to the ones outlined in 3.3 with the exception that the launcher is attached atop the transport vehicle rather than on the ground. The pin-release variants operate differently. Instead of requiring a rail to launch from, they are either held down by a cable on a short flat rail or pinned at a single attachment point, and when the cable or pin are released, the aircraft can operate under its own power. For these pin-release methods, the vehicle must drive up to the 
takeoff speed of the aircraft before triggering the release, which is typically at least $20 \%$ greater than the aircrafts stall speed. This is generally done using a retracting actuator (Figure 22) or by pivoting past a release point (Figure 23).

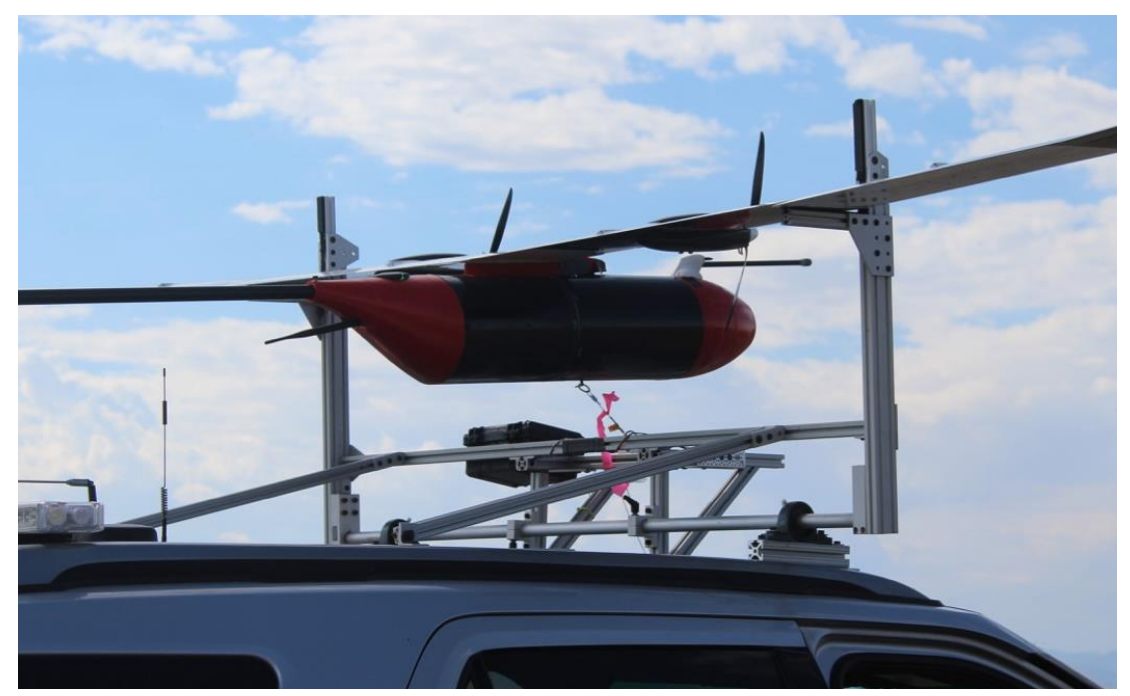

Figure 22: Roof-Mounted Cable Release Example (University of Colorado Boulder)

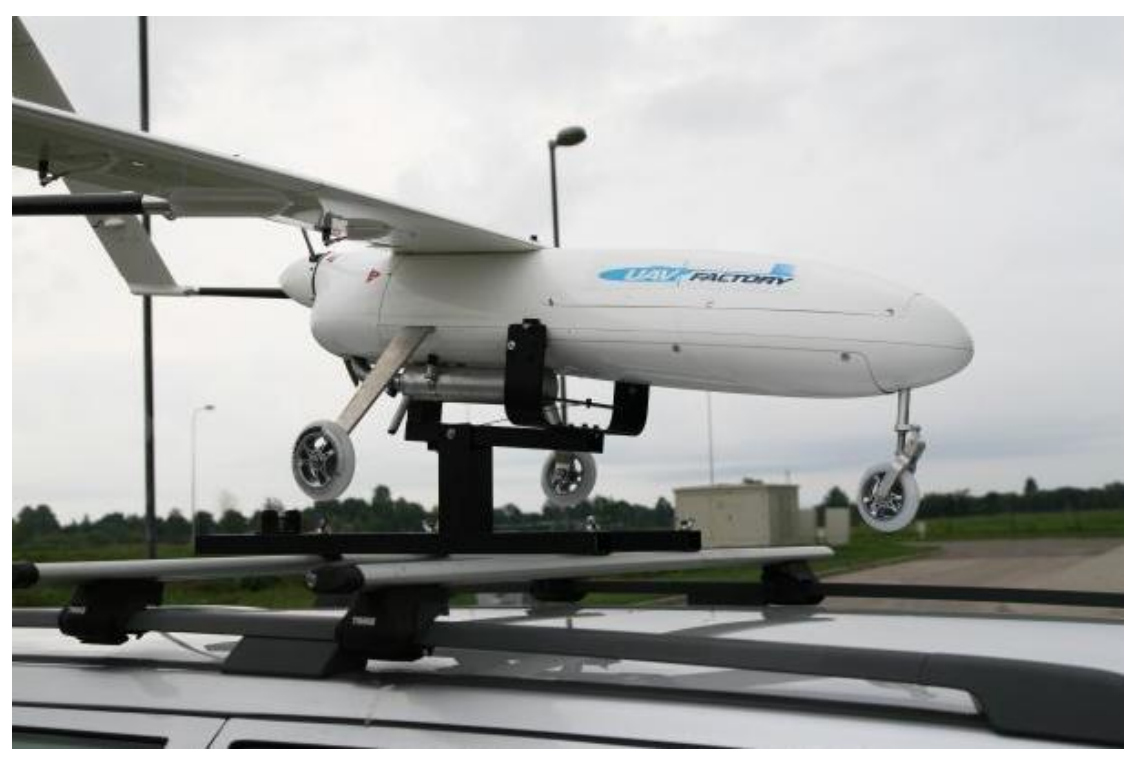

Figure 23: Pivoting Pin Release Example (UAV Factory) ${ }^{35}$ 
The catapult-based systems have the same pros and cons as in section 3.3, with the exception of the cons regarding transportation space requirements. For transport, due to the angle they must be set at for launch, they are typically collapsible on top of the vehicle with a simple pop-up support to maintain the launch angle. This style system drastically improves on the ground-based catapult, but still is not as ideal due to the relative complexity of the system when compared to the pin-release style launch style.

The pin-release system as a whole maintains reliability with minimal moving parts, which makes it a preferable choice. The UAV Factory style of pivoting pin release is a novel approach to the problem, but since it cannot easily be fashioned to work with other aircraft and would take much testing to replicate, it has been discarded.

That leaves the cable style pin-release as the final version of the roof-mounted launcher for consideration, which has many pros with few cons. This style of system can be easily modified to work with any aircraft (change the middle crossbar length as wingspan requires), the speed of the launch vehicle can be changed to account for wind gusts and cross-wind conditions, can be used in all-weather conditions, and as a plus, integration of ambient weather sensors to ensure aircraft sensors are reading accurately is easy (extra 8020 extension housing the sensors). The only modification to the aircraft is a single small hole for a strong cable to pass through (one end looping around spar, the other around a linear actuator on the frame) to hold the aircraft onto the launcher. As a whole, the design and build can be very simple by building out of 8020 aluminum extrusions.

The primary con to this system is that there will be a short length of cable that will trail under the aircraft at all times during flight. This can pose an issue for some single pusher prop (propeller behind airframe) aircraft. The way to circumvent this issue is to use a short enough length of cable that there is no possible way for the cable to ever interfere with the propeller. One 
might also think this would cause an issue with belly landing, the landing speed is slow enough and the cable free of large enough loops to grab anything that there is no foreseen issue with a dangling cable, which would likely be a thin Kevlar rope. Due to the actuator holding the aircraft down while the vehicle is approaching the takeoff speed of the aircraft, some airframes may require a minor reinforcement to ensure the cable does not rip out of the aircraft. Most already have sufficient supports in the spar, but others might require a small wooden plate to ensure the aircraft is not damaged. The final drawback to this system is most roads are oriented either NorthSouth or East-West. This means if a North West or other off axis wind were blowing at the launch site, the vehicle will need to reach a higher speed prior to launching the aircraft.

\subsubsection{5: Chosen Design Class}

The design that was chosen was the cable pin-release roof-mounted launching system. Of the options, it would be the quickest to design, test, and be flight ready using minimal cargo space while satisfying the design goal of operating under a severe weather chasing scenario. Parts are also much less likely to fail under various outdoor conditions than the other primary candidate, the roof-mounted catapult system. One example of why that system would not be ideal is the system will be tested under various temperature conditions, and the bungees required by the perform differently in hot vs cold weather. This is intrinsic to the elastic bungees themselves, and unless very expensive bungees are used, no other bungee will be able to appropriately resolve this problem. As such, the cabled pin-release launcher was chosen due to simplicity of design, speed of manufacture, ease of operation, and reliability of results.

\subsection{2: Design of Launcher}


The final conceptual design was a variation of the UC Boulder cable-release roofmounted system. The design was a simplistic system with minimal moving parts (less prone to failure), would not require internal vehicle storage (minimal internal volume footprint), and would work for a wide variety of aircraft rather than a single type. A bungee or catapult system must have separate conditions for heavy and lightweight aircraft, which increases complexity compared to simply driving a little faster prior to release. This design concept also implemented simple wing support structure and was capable of installing a weather station with visual readout in the cab, allowing for ambient monitoring without extra ground station equipment.

The main portion of the frame was built using 25 series 8020 extruded aluminum bars. Doing some back of the envelope (BoE) calculations, the approximate loading on the frame from the wind alone was calculated. The below equation describes a simplified estimation of forces exerted on the launcher's frame by a constant velocity free stream wind.

$$
F_{w}=\frac{\rho V^{2}}{2}\left[A_{\text {aircraft }} C_{D_{\text {aircraft }}}+A_{\text {launcher }} C_{D_{\text {launcher }}}\right]
$$

Where $F_{w}$ is the total force exerted on the launcher structure while the launch vehicle is moving, $\rho$ is the air density, $V$ is the velocity, $A_{\text {aircraft }}$ is the wetted surface area of the aircraft, $C_{D_{\text {aircraft }}}$ is the total drag from the aircraft on the launcher, $A_{\text {launcher }}$ is the frontal area of the forward launcher structure, and $C_{D_{\text {launcher }}}$ is the estimated total drag from the forward launcher structure. The aluminum bars are assumed to be flat plates for drag purposes. For robustness, a factory of safety of 4 was used in the final load calculation. Assuming TIA is mounted on the launcher, and assuming a total frontal cross-sectional area of $90 \mathrm{in}^{2}$ for the launcher with a $C_{D_{\text {launcher }}}=2.1$ (flat plate drag) ${ }^{36}$, the total force imparted on the launcher is 31 pounds. With the factor of safety applied, this was then rounded up to 125 pounds. The final load value was then inserted to 8020.net's online deflection calculator ${ }^{37}$, which uses the true dimensions for 
moments of inertia as well as the actual modulus of elasticity and yield strength of each material type and shape. By utilizing the worst-case loading on the vertical beams (point load at the opposite end of a beam with one fixed point) on the tallest vertical structure ( 22 inches) the max deflection of the frame was estimated to be approximately 1". Assuming the more realistic evenly distributed loading, the deflection is reduced to roughly 0.4 ". If the factor of safety is removed, these values become 0.28 " and 0.10 ", respectively. These calculations are assuming there is no rear supports to help prevent deflection of these vertical members, which the actual design utilizes to prevent this flex. Running the same calculations for the total estimated wind force on the launcher without the aircraft mounted (24 pounds force, rounded to $100 \mathrm{lbs}$ with factor of safety), the max deflection assuming a point load the end is 0.84 " while the deflection assuming an evenly distributed load is 0.31 ". Without the factor of safety, the point load deflection with aircraft off the launcher is 0.22 " while the evenly distributed loading causes a deflection of 0.08 ". This was determined to be sufficient travel as that portion will be supported by the rear legs, which help carry that load and reduce the actual travel. As the only time the launcher would ever reach speeds higher than $50 \mathrm{mph}$ would be when the aircraft is removed, which eliminates a significant portion of the loading off the launcher, this configuration was chosen to start. 

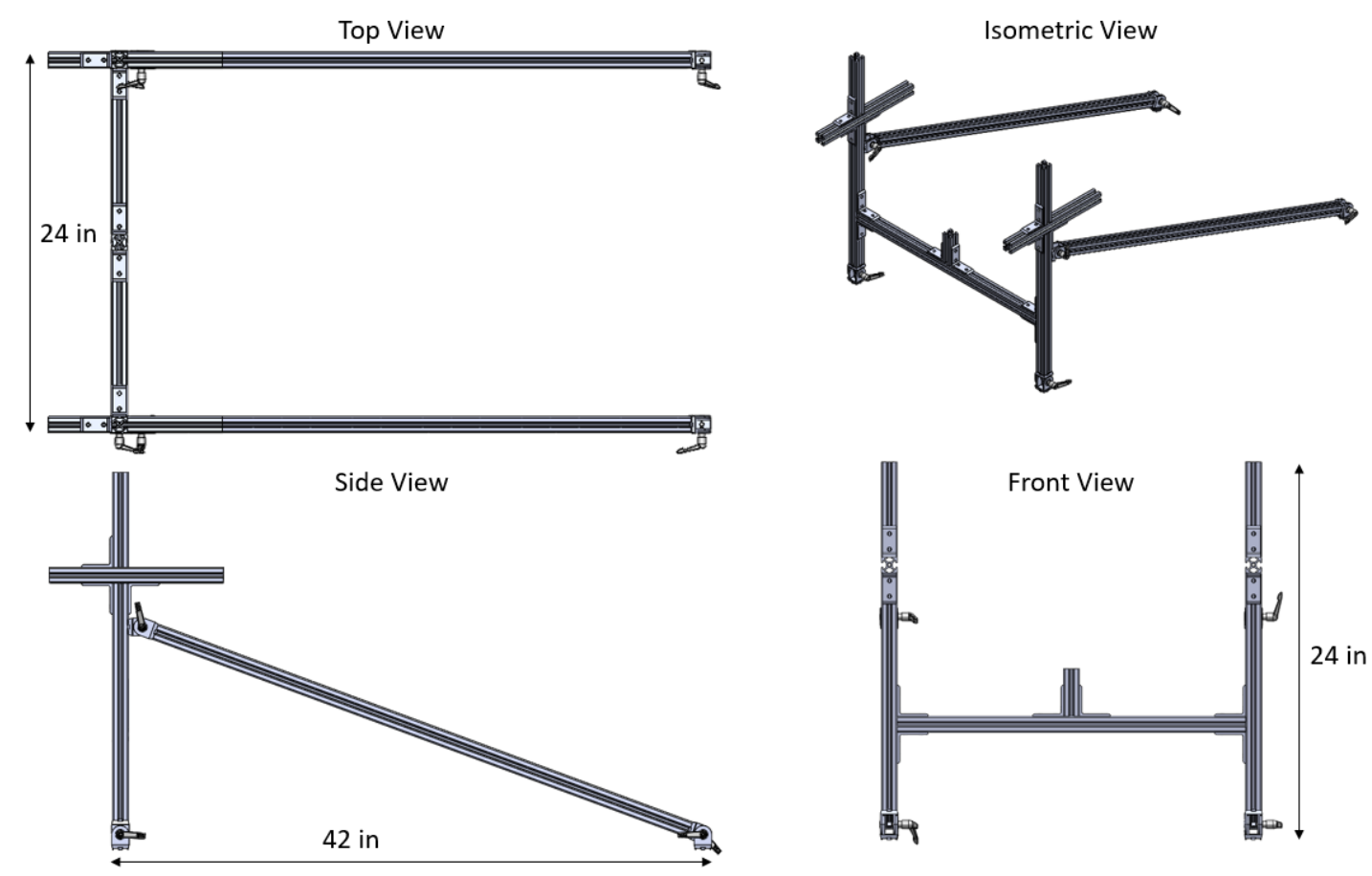

Figure 24: Initial build of the launcher

With the 8020 bars sized, the next major consideration was how to fasten the bars to the vehicle. The launch vehicle requires a T-slot cargo rail to be installed, of which the Thule AeroBlade crossbars were chosen based on the reliability of the Thule brand and the ease of install. Custom T-nuts were fashioned to slide in the rails to anchor the launch system. The launcher itself features 25 series 8020 pivots, which will anchor to the custom T-nuts. The 8020 pivots will then bolt into the profile end of the primary 8020 bars, effectively securing the launcher to the cargo rails of the vehicle.

Pivots will be located at the junction between the rear legs and the main vertical legs, allowing for the exact angle to change depending on where the pivot base is located on the main vertical leg. This allows for easy disassembly and prevents a fixed angle case, which means only one location on the main vertical leg will allow the launcher to function properly. Using a pivot allow for any location to work so long as the front leg is vertical. 
The center crossbar will be secured by use of T-plates that are primarily bolted to the main vertical legs with the secondary flange securing each end of the crossbar. The wing support rails will be supported in a similar way, using T-plates primarily secured to the wing support bar with the secondary flange securing to the top of the main vertical legs. The weather station boom will use two corner brackets, one on each side, to keep it secured to the side of one of the main vertical legs. The linear actuator will use two T-nut plates to bolt to the front of the crossbar. The fuselage support will use a series of 4 corner brackets to keep a 4" 8020 bar secured above the center crossbar.

A synopsis of the build process, including flight testing and ensuing modifications, is outlined in Section 4.3.1.

\section{6: Controls Concepts and Methods}

In order to approximate how the aircraft should handle and perform in severe weather (random direction and intensity of wind gusts), there is a need to determine a method of analyzing the stability and control of the aircraft. This is needed for several reasons. First, it's generally a good idea to have a control model of the aircraft to gain an understanding of how it will react under various ambient conditions. By having this, some potential errors have a chance at being resolved before the aircraft ever takes flight. The second reason is if the aircraft in flight performs similarly to the control model outputs, then the control model could be used to approximation to show to what extent the aircraft could theoretically survive in. At the surface, this insight seems as though it could prove incredibly valuable for approximating performance. However, expectations must be tempered as it is only a theoretical approximation. A control model is only 
as accurate as its approximations and assumptions, and these in general are good enough to view trends but not necessarily used to predict anything exact.

\subsubsection{General Controls Concept}

The overall approach taken for this analysis is a compilation of other strategies taken from various textbooks and academic papers. The general process included first finding pertinent characteristics from the biconvex airfoil $\left(\mathrm{C}_{\mathrm{l} \alpha}, \mathrm{C}_{\mathrm{m} \alpha}, \mathrm{C}_{\mathrm{d} \alpha}\right)$ using a software package, such as XFoil (or similar), and converting those metrics from a 2-D airfoil to a 3-D wing approximation. This

conversion was done by hand using equations shown in Nelson, Raymer, and other aircraft design and performance textbooks, and these converted metrics were compared to results gathered from actual flight performance metrics. These two were used as opposed to a single one to ensure the validity of data sets were consistent even across multiple data acquisition methods. Once the 3-D wing was characterized, an attempt would need to be made to take the aircraft parameters and create an aerodynamic model of the aircraft in the form of matrices. Process steps to do so are outlined in Nelson, and as such were used as a guideline in creating the aerodynamic model for this aircraft. Once the aerodynamic model is created, it could then be run through various algorithms to check for lateral and longitudinal stability and control, as well as run through simulations to test the handling and performance through gust fields.

Since the airfoil and all other aircraft design factors were already determined and produced, the analytical methods were not conducted to drive a more efficient design, but rather to simply analyze the current design's performance. XFoil was chosen to be used to find the 2-D characteristic of the biconvex airfoil. While XFoil has many strengths, solving for airfoils with a sharp leading edge tend to fail in its solver. This is due to the lack of curvature at the leading 
edge, which is required for the solver. This can be circumvented by customizing the airfoil in the XFoil airfoil creation feature to modify the base airfoil to have an infinitesimally small amount of curvature at the leading edge. Doing this actually makes the airfoil closer to how the real airfoil exists on the aircraft, as manufacturing required a slight ogive at the leading edge to bond properly. XFoil would need to be run in both viscous and non-viscous settings at a RE of $1 \mathrm{e} 6$ and Mach of 0.15 to generate appropriate values of lift and get a rough estimation of drag at the target conditions, which was chosen to be the cruise flight condition of $100 \mathrm{mph}$.

Other solvers than XFoil could also have been used to generate these initial 2-D parameters. Athena Vortex Lattice (AVL) is another Fortran-based software package like XFoil that allows for similar data collection. The benefits to AVL are it can also do analysis over 3D objects and include inertia considerations, allowing for some stability derivatives to be derived. Using this inertia solver has many bugs and was not able to be used properly, so while it was not used, it is in theory a very viable tool to gather first approximations of stability and control derivatives.

Once XFoil data runs are complete, the data would then be compiled in Microsoft Excel and run through equations set out in Raymer and Nelson to convert 2-D airfoil characteristics into 3-D wing characteristics. Since the end-goal is to create a set of aerodynamic matrices, not all parameters were sought in the 2-D to 3-D conversions. Some important parameters that were found, among many others, are: $\mathrm{C}_{\mathrm{L} \alpha}, \mathrm{C}_{\mathrm{D} \alpha}, \mathrm{C}_{\mathrm{M} \alpha}, \mathrm{C}_{\mathrm{L} 0}, \mathrm{C}_{\mathrm{D} 0}$. These are general metrics that are useful for comparing aircraft performance. Many other parameters exist, but these were found using formulations derived by Raymer.

With the 3-D parameters determined and verified using both data sets (analytical, flight testing), the next step was to convert the data into matrices to generate a plant dynamic model. These matrices take the form of: 


$$
\begin{aligned}
\mathbf{A} & =\left[\begin{array}{cccr}
X_{u} & X_{w} & 0 & -g \\
Z_{u} & Z_{w} & u_{0} & 0 \\
M_{u}+M_{\dot{w}} u_{0} & M_{w}+M_{\dot{w}} u_{0} & M_{q}+M_{\dot{w}} u_{0} & 0 \\
0 & 0 & 1 & 0
\end{array}\right] \\
\mathbf{B} & =\left[\begin{array}{cc}
X_{\delta} & X_{\delta_{T}} \\
Z_{\delta} & X_{\delta_{T}} \\
M_{\delta}+M_{\dot{w}} Z_{\delta} & M_{\delta_{T}}+M_{\dot{w}} Z_{\delta_{T}} \\
0 & 0
\end{array}\right]
\end{aligned}
$$

Figure 25: Longitudinal aerodynamic matrices ${ }^{43}$

Each of these matrix data points involve a bit of calculus to solve, as is seen below from Nelson:

$$
\begin{aligned}
X_{u} & =\frac{-\left(C_{D_{u}}+2 C_{D_{0}}\right) Q S}{m u_{0}} & X_{w} & =\frac{-\left(C_{D_{\alpha}}-C_{L_{0}}\right) Q S}{m u_{0}} \\
Z_{u} & =\frac{-\left(C_{L_{u}}+2 C_{L_{0}}\right) Q S}{m u_{0}} & Z_{\dot{w}} & =C_{Z_{\alpha}} \frac{\bar{c} Q S}{2 m u_{0}^{2}} \\
Z_{w} & =\frac{-\left(C_{L_{\alpha}}+C_{D_{0}}\right) Q S}{m u_{0}} & Z_{\dot{\alpha}} & =u_{0} Z_{\dot{w}} \\
Z_{\alpha} & =u_{0} Z_{w} & Z_{\delta_{e}} & =C_{Z_{\delta_{e}}} \frac{Q S}{m} \\
Z_{q} & =C_{Z_{q}} \frac{\bar{c} Q S}{2 m u_{0}} & & \bar{c} Q S \\
M_{u} & =C_{m_{u}} \frac{\bar{m}}{I_{y} u_{0}} & M_{\dot{w}} & =C_{m_{\dot{\alpha}}} \frac{\bar{c}^{2} Q S}{2 u_{0}^{2} I_{y}} \\
M_{w} & =C_{m_{\alpha}} \frac{\bar{c} Q S}{I_{y} u_{0}} & M_{\dot{\alpha}} & =u_{0} M_{\dot{w}} \\
M_{\alpha} & =u_{0} M_{w} & M_{\delta_{e}} & =C_{m \delta_{e}} \frac{Q S \bar{c}}{I_{y}} \\
M_{q} & =C_{m_{q}} \frac{\bar{c}^{2} Q S}{2 I_{y} u_{0}} & &
\end{aligned}
$$

Figure 26: Force and moment equations for longitudinal aerodynamic matrix set ${ }^{43}$

Once the general plant dynamics model is created, the next step is to augment the plan dynamic matrices with the gust response of the aircraft. Doing this will allow for the gust simulation and modeling to work properly. This augmentation and gust modeling is done using steps outlined in 
Moeder and Calise's 1985 and 1987 control papers ${ }^{46,47}$. The algorithm was to be run in Matlab using Simulink to help run the simulation space of the gust response.

There was no specific reason as to why Moerder and Calise's algorithmic approach was used over other options aside from the ability to isolate a single axis (lateral or longitudinal instead of both together). Their approach uses a numerical algorithm to calculate optimal output feedback gains for an output feedback control augmentation system. This approach was chosen not only due to the ease in converting into a useable Matlab/Simulink formulation, but also for the ability to isolate a single control plane (lateral or longitudinal). Many obstacles were anticipated in creating the aerodynamic augmented control model, and as such the ability to use just one form was preferred in the event only one set was to be used. 


\section{CHAPTER IV}

\section{SYSTEM INTEGRATION, BUILD OF AIRFRAME AND LAUNCHER}

Prior to building a new airframe, testing of all systems in an old airframe was completed to minimize risk to the new aircraft. This was done by testing the turbine-autopilot interactions on the ground followed by flight tests of the prototype aircraft. Lessons learned from these experiences drove the overall redesign process of the new airframe. Included in this redesign are water shielding considerations, quality of life upgrades for the airframe, and overall integration of systems.

Mylar was also placed in the rear to shield the Pixhawk, GPS, and Aura 8 from any water that gets forced backwards by the inlet and jet pipe. All the servos are water-resistant and are more than capable of surviving water droplets, though the hatches are still "sealed" using tub sealant. Show that should a system or component fail, each component is easy to swap out compared to old designs.

\subsection{Aircraft Flight System Integration}

The next phase was to begin integrating the components into one of the original Diamondback airframes. This was executed prior to manufacturing a new airframe to capture all possible design revisions that might have been required from the additional avionics and electrical systems. 


\subsubsection{System Ground Testing}

The primary goal after having all components selected and balanced was to do a full system test to ensure the aircraft would be capable of completing its mission with no issues arising from the interactions of the electrical and mechanical systems. This series of testing was completed in steps, beginning with the DragonLink RC transmission (without Pixhawk), then adding Pixhawk to the loop, then adding the FPV video link. A full wiring diagram of the final setup is included at the end of this section. The rest of this section details the use of each individual component and how each ties together to create one entire system.

\subsubsection{DragonLink $R C$}

The base Diamondback aircraft was flown using a Futaba T14SG transmitter with a Futaba R7003SB receiver. This receiver was implemented using the SBUS port to send all RC signals to the Aura 8 rate gyro with the exception of throttle, which was sent to the turbine's ECU directly via Channel 3 on the receiver. The DragonLink was able to be setup in the exact same way, with SBUS out going to the Aura 8 for signal mixing and output while throttle (also Channel 3) was sent directly to the turbine's ECU. In order to transmit the RC signals to the DragonLink receiver on board the aircraft while using the same Futaba RC transmitter, a DragonLink transmission "backpack" was installed to the back of the Futaba transmitter, which uses a cable connecting to the back of the Futaba transmitter for direct signal acquisition by the DragonLink system to be broadcast to the on-board DragonLink receiver. The DragonLink receiver was placed in the same physical location as the Futaba receiver was in the previous iteration, allowing for the same wiring to be used between the two systems should there need to be an interchange for any reason in the future. 
When powering the system, control surface deflections were checked for response time with no discernable lag associated with the DragonLink alone. The turbine was also fired up and ground tested under the control of the DragonLink with no issues. This confirms that the DragonLink on its own is not a cause for concern so long as the rest of the avionics and control systems are properly installed. With this step completed, the next part of the integration process as to install the Pixhawk autopilot system.

\subsubsection{Pixhawk Autopilot}

Though the DragonLink system was a simple process, the Pixhawk integration proved to be more of a challenge. As opposed to most sUAS which rely on only one flight controller/gyrostabilizing system, this aircraft utilizes two in series. The Pixhawk was placed in-line between the DragonLink receiver and the Aura 8 rate gyro. The immediate concern with this setup is how a clean signal from the receiver going through two separate control gain outputs is properly applied to the servos to maintain a target state of flight. This is done by only having one control system active at a time, which is controllable through a single switch on the pilot's RC transmitter. When the switch is in the Manual position, the Aura 8's rate gyros are all active while all the Pixhawk's rate gyros and other stabilizing components are disabled, allowing for full RC pass-through of the signal directly to the Aura 8 to the servos for control. When the switch changes states from Manual to any other position, the Aura 8's rate gyro is disabled while maintaining the signal mixing ratios, giving full control authority to the Pixhawk for stability and control systems to take effect. Though this is a more complex method of control, it reduces the amount of customization required of the Pixhawk itself, as it outputs controls to the Aura as if it were a standard tube and wing aircraft using one channel for two aileron servos, one channel for one rudder servo, and one channel for one elevator servo. The Aura then takes these three input signals, mixes them at a predefined ratio (determined through much flight testing), and outputs 4 separate channels: one 
for each elevon servo, one for the yaw thrust vectoring servo, and one for the pitch thrust vectoring servo.



Figure 27: Final wiring diagram of combined systems 
When this system underwent control surface deflection tests, there was not only a slight but noticeable lag, but the servos no longer exhibited "fine-tuned" control movements. The lag was primarily due to lack of direct power source to the servo rail of the Pixhawk. The Pixhawk will output $5 \mathrm{~V}$ to the servo rail, but when under full load, the actual power output is lowered to the point the servos do not reliably respond rapidly (delay up to 2 seconds). This was circumvented by applying a $6.6 \mathrm{~V}$ power source directly to an empty port on the Pixhawk's servo rail. This gave the servo rail sufficient power to maintain proper servo response speed regardless of the power draw required by the servos.

The lack of resolution in the servo control was an unintended consequence of using the Pixhawk autopilot. A servo is typically operated by using pulse-width modulated (PWM) signals with pulse widths between 1 millisecond and 2 milliseconds which corresponds to each of the endpoints of the servo, which are typically $0^{\circ}$ and $180^{\circ}$, respectively. The decimal value of the PWM signal determines what finite angle the servo arm is commanded to achieve. With the Pixhawk system installed, the resolution of that decimal appears to have been reduced, as the servos began to operate between a much lower total number of obtainable angles as compared to a straight RC system. The exact reduction was not measured, as it was not reduced enough to prevent safe operation of the aircraft, but there was a noticeable decrease in the angular resolution of the servos output. This was held true for all four servos.

As a side note: most RC systems define the PWM output range to be $1100-1900$ so that the servo motors, which have a max range 900-2100, are never fully maxed, which help prevent burning the servos out. The Pixhawk, however, allows for a range of 900-2100, which give full actuation control to the Pixhawk. After much testing to verify no ill effects of having the Pixhawk, Aura 8, and DragonLink RC components in series, it was determined there was minimal downside to having them paired. The testing all involved the same servo setup, which included a servo with a protractor attached. The programmer was used first to determine what the 
max achievable range of the servo was, followed by using a DragonLink V3 RC system through a Futaba RC transmitter to get pure RC output values. After these two baseline metrics were taken, an Aura 8 rate gyro SBUS hub was installed between the servo and RC receiver to track how the Aura modifies the signal output when changing Output Tunings and Pulse Output Settings. The same was then done with the Pixhawk, replacing the Aura as the system between the servo and RC system. The only Pixhawk setting changed was the Pixhawk Output range, but both the input and output PWMs were tracked. Finally, both the Aura and Pixhawk were placed in series between the servo and RC system just as it is on TIA: RC - Pixhawk - Aura - Servo. A table summarizing the results are shown below. Only the left deflections are shown due to ease of reading the change in angular deflections, but the same deflections and PWM scales are seen in right deflections. 


\begin{tabular}{|c|c|c|c|c|c|c|c|}
\hline Test & Measurand & Center & $50 \%$ Left & $100 \%$ Left & $\begin{array}{c}50 \% \text { Diff } \\
\text { from Center }\end{array}$ & $\begin{array}{c}100 \% \text { Diff } \\
\text { from Center }\end{array}$ & Comments \\
\hline \multirow{2}{*}{$\begin{array}{c}\text { Servo } \\
\text { Programmer }\end{array}$} & Angle & 90 & 120 & 150 & 30 & 60 & \multirow{2}{*}{$\begin{array}{l}\text { Pure signal movement from a } \\
\text { programmer (max range of servo). }\end{array}$} \\
\hline & PWM & 1500 & 1200 & 900 & -300 & -600 & \\
\hline Pure RC & Angle & 88 & 109 & 131 & 21 & 43 & $\begin{array}{l}\text { Movement via signals directly from RC } \\
\text { transmitter/reciever. }\end{array}$ \\
\hline \multirow{2}{*}{$\begin{array}{c}\mathrm{RC} \rightarrow \text { Aura } \\
\text { (Stock Settings) }\end{array}$} & Angle & 89 & 101 & 115 & 12 & 26 & \multirow{2}{*}{$\begin{array}{l}\text { Servo signal passing from } \mathrm{RC} \text { reciever } \\
\text { through Aura } 8 \text { with default settings to } \\
\text { the servo. Large reduction in angular } \\
\text { deflection at stock settings. }\end{array}$} \\
\hline & Aura PWM & 1501 & 1378 & 1241 & -123 & -260 & \\
\hline \multirow{2}{*}{$\begin{array}{l}\mathrm{RC}->\text { Aura } \\
\text { (TIA Settings) }\end{array}$} & Angle & 89 & 108 & 128 & 19 & 39 & \multirow{2}{*}{$\begin{array}{l}\text { Combining the two settings changes: Pulse } \\
\text { setting is the dominant factor with Output } \\
\text { Tune not having a noticeable effect. } \\
\text { Grants near Pure RC angles. }\end{array}$} \\
\hline & Aura PWM & 1501 & 1316 & 1112 & -185 & -389 & \\
\hline \multirow{3}{*}{$\begin{array}{c}\text { RC } \rightarrow \text { Pix } \\
\text { (Stock Params, } \\
\text { Aileron) }\end{array}$} & Angle & 89 & 109 & 130 & 20 & 41 & \multirow{3}{*}{$\begin{array}{l}\text { Servo signal passing from } \mathrm{RC} \text { reciever } \\
\text { through Pixhawk with default parameters } \\
\text { to servo. Follows the Pure } \mathrm{RC} \text { output very } \\
\text { closely, with margin of error causing the } \\
\text { only noted deviation. }\end{array}$} \\
\hline & Pix PWM In & 1518 & 1307 & 1099 & -211 & -419 & \\
\hline & Pix PWM Out & 1500 & 1299 & 1100 & -201 & -400 & \\
\hline \multirow{3}{*}{$\begin{array}{c}\text { RC } \rightarrow \text { Pix } \\
\text { (TIA Params, } \\
\text { Aileron) }\end{array}$} & Angle & 89 & 105 & 121 & 16 & 32 & \multirow{3}{*}{$\begin{array}{l}\text { Servo signal passing from RC reciever } \\
\text { through Pixhawk with TIA parameters to } \\
\text { servo. Reduced servo output range } \\
\text { compared to pure RC. This to prevent the } \\
\text { servos from oversaturating in the } \\
\text { combined system when in auto control. }\end{array}$} \\
\hline & Pix PWM In & 1518 & 1309 & 1099 & -209 & -419 & \\
\hline & Pix PWM Out & 1500 & 1310 & 1183 & -190 & -317 & \\
\hline \multirow{3}{*}{$\begin{array}{c}\mathrm{RC} \rightarrow>\text { Pix } \rightarrow \text { Aura } \\
\text { (All Stock } \\
\text { Params) }\end{array}$} & Angle & 90 & 104 & 117 & 14 & 27 & \multirow{3}{*}{$\begin{array}{l}\text { Appears that running all default params } \\
\text { results in a } 55 \% \text { reduction in angle at a } \\
\text { given PWM compared to the programmer } \\
\text { and a } 37 \% \text { reduction compared to the } \\
\text { Pure RC test. Aura } 8 \text { at stock settings is } \\
\text { the limiter as seen from its above test. }\end{array}$} \\
\hline & Pix PWM Out & 1500 & 1300 & 1100 & -200 & -400 & \\
\hline & Aura PWM & 1490 & 1359 & 1219 & -131 & -271 & \\
\hline \multirow{3}{*}{$\begin{array}{c}\mathrm{RC} \rightarrow \text { Pix } \rightarrow \text { Aura } \\
\text { (Direct From TIA } \\
\text { System) }\end{array}$} & Angle & 89 & 114 & 142 & 25 & 53 & \multirow{3}{*}{$\begin{array}{l}\text { Combining the Pixhawk and Aura } 8 \\
\text { parameters (as measured from TIA } \\
\text { integrated system with same test servo) } \\
\text { resulted in values slightly lower than from } \\
\text { the output from the programmer, but } \\
\text { greater than Pure RC values. }\end{array}$} \\
\hline & Pix PWM Out & 1500 & 1341 & 1183 & -159 & -317 & \\
\hline & Aura PWM & 1498 & 1244 & 995 & -254 & -503 & \\
\hline
\end{tabular}

Table 4: Tracking of PWM signals through each subsystem at stock and final parameters

From this testing, it is clear to see that though each system does respond differently to varied setting changes, the overall result is that by increasing the PWM output range, the max deflection is also increased for a given input RC signal. In individual system tests, regardless if the new max range is applied to only the Aura or to the Pixhawk, the same angle can be achieved by changing just one of the systems to max range. As the last data set shows, if both are changed to the max, an even larger amount of deflection can be achieved, which is the setting the aircraft is set for. The integrated system deflection could easily match the servo programmer's max deflection 
value, but the reduction was intentionally set to ensure the servo does not over-extend and burn out. This allows the servo to have slightly stronger throw in the reduced range compared to the true max range and helps prevent the servo from ever reaching a locked-out state, which would cause an aircraft to crash.

Though the servo integration proved fairly easy to integrate into the Pixhawk-Aura-RC system, this combination proved especially troublesome for the turbine. The primary difference between the servos and the propulsion system is the servo signals went through both Aura and Pixhawk, whereas the turbine signals only go through the Pixhawk, giving a total path of RCPixhawk - ECU. Though there is one less degree of extra signal processing and output, this still proved especially troublesome for the turbine, with some errors immediately noticeable via ground tests. The biggest ground-test error was getting the trim to apply properly. As mentioned above, the Pixhawk does not necessarily do a direct signal pass through, there is light signal processing done to any signal that is not setup for pure RC pass-through. As the goal for the system is full autonomous mission pathing, the goal was to not use any RC pass-through channels and instead use the appropriate modified signal output channels. The table above shows the difference between the modified and pure signal output, which, in the above testing, can vary by as much as 18 PWM for center trim, 8 for $50 \%$ throw, and 1 for an endpoint.

For the Kingtech turbines, a Learn RC is usually done before the first flight of the day to ensure the ECU is able to interpret the signals received by the transmitter during flight. The turbine, when conducting a Learn RC, records the absolute max and min PWM values, meaning it requires max and min trim position at the respective endpoint conditions (trim at -50 while the stick is at $-100 \%$, trim at +50 while stick is at $+100 \%$ ). Since $0 \%$ throttle is normally associated with stick down, that left the idle throttle PWM as 1100. Both the turbine startup and shutdown processes are governed by the trim position. For positive trim values, the idle is incrementally increased, allowing for the turbine to startup. When the trim goes below the neutral point (no 
throttle trim) the turbine enters the shutdown sequence. This means that any fluctuation around the 1100 PWM mark has a chance to influence the turbines behavior, especially since trim tabs are a fine-tune adjustment to the PWM signal. The throttle trim tab on the RC transmitter modifies the PWM signal by a max value of \pm 126 ( $\pm 10 \%$ of signal) with a linear scaling between the endpoints. However, there also is an offset generated by the Pixhawk due to the parameter settings. Because of this, the default Pixhawk settings were not compatible with the turbine. The turbine would fire off on the ground, but it was difficult to get the turbine to enter shut down as the Pixhawk does not allow for PWM outputs beyond the defined range. Because of this, the minimum PWM output in the Pixhawk was updated to have a lower minimum than the rest of the channels, which fixed this issue. Below is a table showing the trim tab values (max is $\pm 50)$ and the corresponding PWM both entering and leaving the Pixhawk in the final working version.

\begin{tabular}{|c|c|c|c|}
\hline \multicolumn{4}{|c|}{ TIA Throttle PWM Values with Change In Trim } \\
\hline Direction & Trim \% & Pixhawk In & Pixhawk Out \\
\hline \multirow{2}{*}{ Up } & -50 & 1225 & 1302 \\
\cline { 2 - 4 } & -25 & 1171 & 1251 \\
\hline \multirow{2}{*}{ Trim } & 0 & 1099 & 1190 \\
\hline \multirow{2}{*}{ Down } & +25 & 1032 & 1130 \\
\cline { 2 - 4 } & +50 & 973 & 1079 \\
\hline
\end{tabular}

Figure 28: Trim tab effect on throttle PWM signal through Pixhawk

The turbine was now capable of starting up and shutting down on command, but all integration testing has been conducted in Manual thus far. When switching from Manual to FBWA, there were a multitude of problems. In FBWA, the pilot still can command a throttle input, but the Pixhawk determines the minimum and maximum throttle positions as well as the slew rate of the throttle signal. Slew rate is the maximum percentage change in throttle per second. An example explaining this is found in the Pixhawk parameter page: "A setting of 10 means to not change the throttle by more than $10 \%$ of the full throttle range in one second". The 
Kingtech turbines that have been used in this aircraft $(\mathrm{K} 45, \mathrm{~K} 70)$ both have approximately an 8 second spool-up time from zero throttle to max, and another 8 second spool-down from max throttle to zero throttle. Since the Pixhawk is usually associated with electric motors, it was set by default for a $100 \%$ slew rate, meaning it was effectively always commanding $100 \%$ or $0 \%$ throttle to hit the target value. In the air this would normally be associated with an airspeed or altitude reading to match, but for the ground tests this was done by changing the throttle percent in the Pixhawk directly. It was noted that the $0 \%$ throttle condition would immediately initiate the turbine shutdown sequence. This would have potentially catastrophic implications if done in the air, so an immediate and full resolution was applied.

The first step was to reduce the Trim Center value of the throttle channel in the Pixhawk to match the minimum PWM value, down from 1100 to 1079. This was done so that when the RC system commands positive trim value (as is required for normal operation), the Pixhawk is guaranteed to have a throttle value above the minimum, preventing an unwanted shutdown sequence from commencing. The second step to preventing the Pixhawk from disarming the turbine mid-flight was to establish minimum, maximum, and "trim" throttle conditions prior to flight testing. Minimum and max throttle values are the min and max percents of the throttle range that the Pixhawk can apply while the "trim" throttle condition is the target percentage of throttle to apply for a normal flight. By making these updates to the Pixhawk Parameters file, the turbine passes all ground tests, allowing for seamless transitions from Manual to FBWA and back to Manual.

To reduce the amount of wiring required for the avionics, the Pixhawk and Aura 8 need to be placed next to each other. Though there is sufficient internal volume around the Aura 8 to accommodate this, the Pixhawk requires a flat and level surface to mount to ensure all readings are accurate, whereas the Aura did not. As such, an extra piece of structure of required to keep the Pixhawk level. This was done in the form of a new tray that slots into the Mid Bulkhead while 
resting against the bottom edge of the Rear Bulkhead, with epoxy being applied at both ends. The slotting ensures the tray has two points of positive lock, which assists the epoxy in ensuring the tray remains rigid. The tray was sized such that both the Pixhawk and Aura 8 could sit on it without interfering with the mounting of the Yaw TV Servo or with the rear hatch from closing. This allowed for the Pixhawk servo rail and the Aura 8's servo rail to be side-by-side, allowing for very short (6") male-to-male cables to make the signal connections. The length was chosen as opposed to any shorter options to allow excess cabling to zip-tie to the Rear Bulkhead, keeping all wiring out of the way of reaching the Aura, Pixhawk, or any other system.

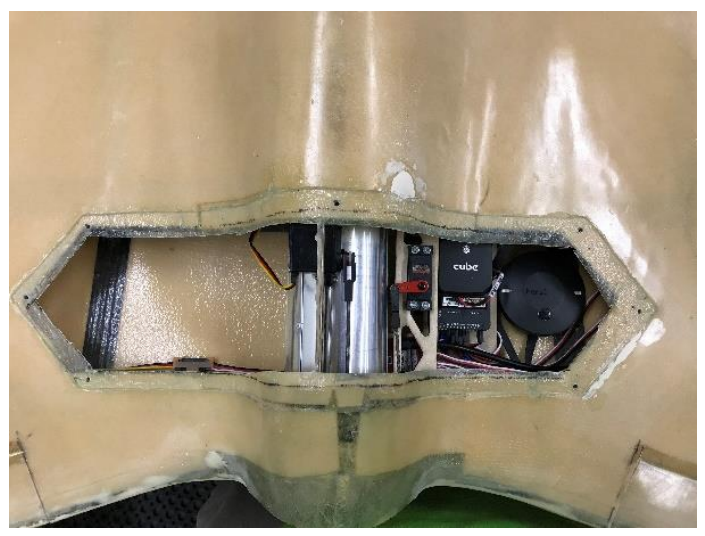

Figure 29: Rear avionics bay of TIA

With the Pixhawk integration resulting in successful pure RC and FBWA control of all avionics and propulsive functions via ground testing, the final point of integration before the first test flight was to integrate the video system.

\subsubsection{FPV Video}

Properly integrating the video system proved to be much less difficult and time consuming than the Pixhawk integration did. This was mostly due to the fact that integrating video systems into aircraft are much more commonly done than integrating a small turbine to an autopilot, of which there are no documented cases of reputable sources doing so. The primary considerations for the video 
system were physical placement of each component and the where to power the systems from. The three hardware components of the video are: FPV camera, video transmitter (VTX), and video antenna.

For the clearest view from the aircraft, the camera was chosen to be placed in the nose. Other considerations were offset from the middle of the nose or in a winglet, but the offset placement would put the camera at risk for damage as it would be slightly sticking out, preventing any chance of keeping the camera safe in a precipitating environment, and the winglets were not quite wide enough to fully house even a micro-sized FPV camera. Both of these options also kept the aircraft in view of the camera, which was deemed less desirable than a pure frontal view from the center of the nose. As such, that location was chose. A hole was cut in the nose to allow for a slightly downward-facing angle for the camera to keep the ground in view during flight so the pilot could maintain situational awareness throughout the flight. If the camera is angled purely horizontal or even slightly upwards, during certain maneuvers the ground can disappear from view, which is the pilots only reference point when flying purely off an FPV video feed.

The camera chosen was a RunCam Split 3 Micro, which features a camera with an integrated DVR system that records all video taken at 1080p to a micro-SD card while sending a live feed via the FPV transmitter at a quality of 720p. Since camera outputs a digital signal, the physical distance from the camera to the FPV transmitter was not an issue. This allowed for the FPV transmitter to be placed in the rear of the aircraft in the optional payload bay. This allowed for easy access to all the video components (namely to change between the two VTX channels if needed) while keeping the distance between the VTX and its antenna minimal. As mentioned earlier, for RF mitigation, the VTX antenna was placed in the Port winglet to keep its radiating signal feed as far away from the other RF transmitters and receivers on board as possible.

The wiring for the FPV system was fairly simple in comparison to the other systems onboard. The camera itself is powered from the VTX, and in the same wiring harness the digital signal of 
the FPV camera is sent to the VTX. The output signal of the VTX goes directly to the antenna in the port winglet. The convenience of this wiring setup is there is only one power input, and that is to the VTX. The VTX draws power from the Pixhawk current sensor. The current sensor intakes power from a battery and acts as a BEC to power the Pixhawk while tracking the current draw on the battery. The other end of the current sensor is another XT60 battery connector, allowing for pure battery voltage and current pass-through. This power pass-through is what powers the camera system.

In order to receive and use the signal on the ground, other antennas near the ground control station (GCS) are needed for the pilot. The antenna must be capable of receiving the $1.3 \mathrm{GHz}$ output signal from the VTX. The GCS antenna is attached to a video receiver, which converts the video into a useable output. This output cable is attached to a video screen to display the footage captured from onboard the aircraft in real-time. From testing the video feed on the ground, the camera was deemed secure in the nose and provided the pilot sufficient situational awareness to give max chance to recover using only the FPV screen if absolutely necessary (emergency situations).

\subsubsection{Flight Testing of Integrated Systems}

After integrating all systems and ensuring no issues remained from ground testing, the next step was to try flying the fully integrated aircraft. Of note, this set of testing was still conducted on the test airframe, not the finalized TIA airframe. This was so that if any issues were to arise from this set of testing, the final aircraft would not be subjected to the potential damage that arises from any failures that might be encountered. From the flight testing, many issues were found that were hidden during flight testing, with most only occurring in autopilot-controlled modes. There was only one source of error that persisted even in Manual mode, and that was an 
intermittent turbine shutdown during flight. Each of the major errors encountered are explained below and the resolutions enacted for each.

\subsubsection{Intermittent Mid-Flight Turbine Shutdown}

The initial turbine used for the test aircraft was a Kingtech K45 Gen 1 turbojet. The jets as a whole were demonstrated to be very reliable, but they had also only ever been tested in full manual mode (no autopilot in the loop), where the raw PWM signal sent from the transmitter was received on board, sent to the $\mathrm{ECU}$, which then sent commands to the turbine. As such, a procedural strategy was implemented, where the aircraft would be launched and intitialy flown in Manual, then slowly cycled through each of the major modes to ensure no major systems errors exist. These major flight modes and their uses are: Manual for takeoff/landing, FBWA for cruise and approach from distance, Loiter to keep the aircraft circling a target point until commanded to move (typical for landing pattern), Auto to execute an uploaded mission, and RTL (return to launch) to have the aircraft return no matter what its previous commands were. These modes are easily toggled on the transmitter using a 6 position switch ( 2 position switch that changes offset for a third switch, effectively giving 6 positions).

Though cycling through flight modes was the goal, an error continued to present itself, even on top of the other errors that have yet to be described. This error came in the form of the turbine dying mid-flight. The exact timing during flight it would shut down was not consistent (some within a minute, some after 7 minutes, sometimes not at all), there was no single mode it most commonly happened in, and there didn't appear to be any reason for this shutdown. The only commonly observed issue with Kingtech turbines came from the BEC used to provide power to the turbine itself, in which case the turbine would fail to start up. Once started, there wasn't an issue with operation of the turbine. That issue was easily mitigated by using a $9.9 \mathrm{~V}(3 \mathrm{~S} \mathrm{LiFe})$ 
rather than a $3 \mathrm{~S}$ LiPo battery on a BEC, which was done long before ever being used in this aircraft. The only clue given was that the ECU was not showing any error codes when reviewing the log files. In each case, the ECU completely loses power, which killed the turbine. Because the turbine was a K45 Gen 1, there was no auto-restart function. Kingtech would eventually go on to release a K45 Gen 2, which featured auto-restart options should a turbine fail mid-flight. This feature, though helpful, still would not sufficiently resolve the issue on its own. This led to a deep investigation into the wiring of the aircraft.

Upon closer examination, there was a ground loop error caused by a lingering artifact from previous testing on the aircraft. Before the airframe was used for this project, there was an attempt to control the ECU power using a MOSFET switch, which would effectively allow for power to the ECU to be cycled on command of the pilot. This was done as a rudimentary method of restarting the turbine mid-flight in the event it died. This MOSFET switch was improperly wired, which would cause the switch to intermittently flip when the power surged, causing the turbine's PWM signal to go to zero, shutting the turbine down. This MOSFET was removed and proper wiring restored, which resolved this issue. Prior to the switch's removal, this problem was prevalent in conjunction to the other flight testing obstacles described in the next subsections.

The resolutions enacted for this issue was to remove the MOSFET switch and upgrade from a K45 Gen 1 to a K45 Gen 2 (auto restart and pump self-priming features) to give an extra layer of safety should other similar issues arise.

\subsubsection{Turbine Surging}

Though no issues involving slew rate were observed through ground testing, the same was not true through flight testing. Ground testing did not reveal errors since throttle is still primarily controlled by the pilot in FBWA, which is where integrated turbine ground testing ended. The autopilot only gains full control of throttle in Auto mode, which has remained 
untested prior to flight testing. In both FBWA and Manual, the turbine mostly flew as expected, with the only real issue having already been described.

However, switching to Auto mode is where issues began to abound for the turbine. Many of the parameters in the Pixhawk were not fully known at the time, so altering any parameter from default values had an enhanced risk of the aircraft experiencing issues, especially since there currently is no documentation on how to run a turbojet from an sUAS autopilot system. As such, all default parameters were used. What was not considered when doing this is how the autopilot was allowed to control throttle inputs.

The slew rate was set (by default) to allow the Pixhawk to change the throttle input by $100 \%$ of the max range per second. This means that in a given second, the Pixhawk could command a $0 \%$ to $100 \%$ throttle, and the next second command a $100 \%$ to $0 \%$ signal. This was problematic, as the turbine takes $\sim 7$ seconds to spool up from idle ( $0 \%$ throttle) to $100 \%$ throttle, and another $\sim 7$ seconds to spool down from max throttle to idle. When in auto mode, the autopilot is set to maintain a given altitude by not only adjusting control deflections but also using throttle. When the aircraft was set in Auto and executing a mission at level altitude, the turbine could audibly be heard cycling between spool up and spool down commands. This was not sufficient for the turbine, so the aircraft was switched back into FBWA for landing approach, then Manual for landing.

Once on the ground, the throttle slew rate (THR_SLEWRATE) was set to 80. This meant the Pixhawk could only command a max change of $80 \%$ of the throttle per second. This slight reduction served to help minimize the surging effect, but still did not negate it completely. This was due to another setting being very low for this aircraft: the trim airspeed (TRIM_ARSPD_CM) setting, which tells the Pixhawk at what the desired trim airspeed is for steady level cruise flight. This was set by default to $1200 \mathrm{~cm} / \mathrm{s}(26.8 \mathrm{mph} / 23.3 \mathrm{knots})$, but for this aircraft a value of $3576 \mathrm{~cm} / \mathrm{s}(80 \mathrm{mph} / 70$ knots $)$ was deemed appropriate as this was the cruise 
speed of the original test aircraft. Despite changing the autopilot's trim airspeed to match the cruise flight condition and the slew rate to prevent full throw of the throttle, the extent of the surging was not completely negated.

After further review of the Pixhawk parameter list, another pertinent variable was found: TRIM_THROTTLE. This setting tells the Pixhawk at what percent throttle the aircraft should stay at in normal flight. This was set by default to $45 \%$, but for this aircraft a value of $75 \%$ was deemed appropriate as that throttle percentage correlates to the normal cruise speed of the initial aircraft of $80 \mathrm{mph}$. (Note: the propulsion system is a turbine with half-expo on the throttle, meaning a $75 \%$ throttle setting is operating the turbine at roughly half the maximum thrust the turbine can output.)

Before explaining how these all tie together, it is important to note one final parameter: TECS_SPDWEIGHT. What this parameter does it tell the autopilot whether to use pitch control surface deflections to focus on a desired altitude (value of 0) or airspeed (value of 2). By default, this is set to 1 , which requires the autopilot to use pitch to account for both altitude and airspeed. This is normally a good thing for aircraft, but since TIA uses thrust vectoring, any change in throttle will change the effectiveness of the trim deflection of the pitch thrust vector. This effect heavily influences the dynamics of why the turbine was surging.

After flight testing, the primary issue with the surging was from the Trim_Throttle parameter, but there were other additive effects from all the settings being tied together in the various control loops. While flying in a cruise condition, the turbine was trying to maintain a throttle position of $45 \%$, which is much too low to obtain the $80 \mathrm{mph}$ cruise airspeed that was set. This meant as the turbine reached the desired airspeed, it would try to throttle down. Upon doing this, the aircraft would slow back down, which required the autopilot to command the turbine to spool back up, which drove a portion of the surging. While that interaction was ongoing, each surge of the throttle also caused an imbalance in pitch, as the pitch thrust vector was set at a slight 
angle upwards. This also meant the aircraft was seeking not only to control airspeed, but now also the altitude. Since the autopilot was to use control surface deflection and throttle to control altitude as well, this meant even if airspeed was matched properly, there would still be turbine surging while the throttle was used to match the desired altitude. By increasing the Trim_Throttle and Trim_Airspeed values to their respective cruise conditions, the throttle was no longer commanding a high-low-high signal, rather it was now commanding fluctuations on the order of $1-2 \%$ throttle. That small amount of fluctuation is trivial, as a pilot manually commanding the throttle position has a similar amount of travel around the desired throttle point. After more flight testing, the aircraft was confirmed to no longer exhibit a throttle surge in any flight mode (Manual, FBWA, Auto, etc.)

\subsubsection{Loss of Altitude During Bank}

During initial flight tests in non-auto flight modes, the pilot was able to balance the aircraft during banking maneuvers in such a way that the aircraft did not gain or lose altitude, rather acting much like a proper coordinated turn. However, when the aircraft was flown purely by autopilot control, the aircraft would consistently start nosing down and losing altitude during a bank. This effect increased as the bank angle increased. To supplement this, several parameters under the Pitch to Servo (PTCH2SRV) category were modified to more appropriately control the aircraft. A summary of these pertinent parameters are found in Appendix A, with the full parameter list found in Appendix G.

\subsubsection{Launching Autonomously From Launcher}

In order for the autopilot to control the aircraft from before the aircraft is even released, it must first know when the aircraft will be released. There are multiple ways to do this. The ones relevant to this project are: minimum acceleration (bungee/pneumatic launchers, 
TKOFF_THR_MINACC) or minimum GPS groundspeed (dynamic launchers,

TKOFF_THR_MINSPD). Each are used for a different class of launcher, but will provide the same end result: arming the motors and attempting to start the auto-mission once the prerequisite condition is met. This helps to minimize the I-gain developed from sitting on the launcher prior to launch, though it's generally recommended to minimize the I-gain in general, as that error drift over time can hinder the aircraft's performance. Should a delay need to be set from when the chosen condition is met (ie bungee or pneumatic launches), another setting (TKOFF_THR_DELAY) sets a delay from the time the condition is met before the throttle is armed. The autopilot attempts to start the auto-mission prior to the arming of the motors, the delay is simply a throttle delay. Settings specific to autonomous launching of the aircraft from the launcher is described in more detail in Section 4.3.2.

\subsection{Aircraft Build}

This section outlines how the aircraft was built. Based on the test aircraft's flight testing, no major structural overhauls needed to take place other than minor movements and tweaks specific for this aircraft. Most notably: 2-core-1 layup of $3 \mathrm{oz} / \mathrm{yd}^{2}$ fiberglass compared to the 1-core-1 2 $\mathrm{oz} / \mathrm{yd}^{2}$ carbon fiber, winglet mounting methods, hatch security, and the reduction in skin cutaway for the nose hatch. Since the general manufacturing process of composite airframes is the same as has been outlined by Daniel Hunt ${ }^{44}$, this section will focus on the specific parameters behind the fabrication of TIA rather than each finite step.

\subsubsection{Airframe Structural Modifications}


Where the methods deviate from what was outlined in Hunt's paper ${ }^{44}$ is in the core material. Composite UAS tend to utilize balsa wood as the core structure, as it is a lightweight material that is relatively easy to form in a mold. A layer of balsa is usually sandwiched between 1-2 layers of fiberglass or carbon fiber on either side. This same overall method was kept the same, except instead of using typical 1/16" balsa wood, a 1/8" thick closed cell foam by Divinicell was used. This was done for a variety of reasons.

First, to form balsa in a mold requires a lot of prep work, as the balsa must first be soaked in water for an extended period of time, then placed on the mold to take shape, then allowed to dry fully, then removed to be glued together using a Cyanoacrylate Adhesive (CA) before finally being trimmed to fit in its final position. This process takes several man hours to complete and requires a significant waiting period to fully complete, as the balsa must first fully soak, then fully air out before used in a layup. By contrast, the Divinicell foam can be formed to a mold by simply applying heat from a heat gun. The foam can be heat-formed to the mold in a matter of minutes, then adhered together using the same CA adhesive, and be ready to be used in a layup as soon as the CA fully dries (30 seconds-1 minute). This negates the two waiting periods the balsa requires, allowing for a more rapid manufacturing process.

Secondly, balsa wood is strongest when loads are applied across multiple grains and weaker when a load is applied along a single grain. This inherent weakness makes it less favorable when the foam, at the same weight, is capable of maintain the same level of strength in all directions instead of just one. This is due in part to the fact that the foam $(1 / 8$ ") is twice the thickness of the balsa (1/16"). Bending stress of a cantilever beam loaded by a point load is found to be:

$$
\sigma_{b}=\frac{3 P L}{2 w t^{2}}
$$


Where $\sigma_{b}$ is the bending stress, $P$ is the normal force, $L$ is the length of the beam, $w$ is the beam width, and $t$ is the thickness of the beam. This implies that the thicker the material is, the stronger it is in resisting bending stresses. Though the foam is not stronger when compared against balsa of the same thickness $\left(1 / 16^{\prime \prime}\right.$ balsa is nearly as strong as $1 / 8^{\prime \prime}$ foam, as seen in Table 5), the foam being twice as thick as the balsa gives an added level of rigidity in the structure that the chosen balsa would not be able to provide while remaining at the same weight, with the foam providing that same level of strength in all directions rather than one.

A set of break test data was conducted to define the difference in strength between these two core materials. In the testing, each section was broken via a point-load break-test along the midline of 3" x 3" test sections comprised of a 1-core-1 layup of different permutations of fiberglass $\left(3 \mathrm{oz} / \mathrm{yd}^{2}\right)$ and carbon fiber $\left(3 \mathrm{oz} / \mathrm{yd}^{2}\right)$ oriented in both $90^{\circ}$ and $45^{\circ}$. To allow the balsa to adequately compare to the foam, the test line was perpendicular to the grains of the balsa. This is because balsa wood is much weaker when a load is placed along the grain than across them, as seen in Table 5. Of note: parenthesis denote break in direction of the balsa grain with all other perpendicular to the grain. All test data was conducted from the same stock of $1 / 8$ " Divinicell closed-cell foam and 1/16" Balsa on a standardized 3" x 3" test section. 


\begin{tabular}{|c|c|c|c|c|}
\hline $\begin{array}{c}\text { Core } \\
\text { Material }\end{array}$ & $\begin{array}{c}\text { Composite } \\
\text { Material }\end{array}$ & $\begin{array}{c}\text { Bias } \\
\left({ }^{\circ}\right)\end{array}$ & $\begin{array}{c}\text { Break Force } \\
(\mathrm{lbf})\end{array}$ & $\begin{array}{c}\text { Test Section } \\
\text { Weight }(\mathrm{g})\end{array}$ \\
\hline \multirow{3}{*}{$1 / 16^{\prime \prime}$} & $\mathrm{cf}-\mathrm{b}-\mathrm{cf}$ & $90-90$ & $29(13)$ & 19 \\
\cline { 2 - 5 } $\mathrm{B}$ & $\mathrm{cf}-\mathrm{b}-\mathrm{cf}$ & $90-45$ & 30 & 21 \\
\cline { 2 - 5 } $\mathrm{A}$ & $\mathrm{cf}-\mathrm{b}-\mathrm{fg}$ & $90-90$ & 17 & 15 \\
\cline { 2 - 5 } $\mathrm{L}$ & $\mathrm{cf}-\mathrm{b}-\mathrm{fg}$ & $90-45$ & 10 & 17 \\
\cline { 2 - 5 } $\mathrm{S}$ & $\mathrm{cf}-\mathrm{b}-\mathrm{fg}$ & $45-90$ & 17 & 18 \\
\cline { 2 - 5 } A & $\mathrm{fg}-\mathrm{b}-\mathrm{fg}$ & $90-90$ & 16 & 15 \\
\cline { 2 - 5 } & $\mathrm{fg}-\mathrm{b}-\mathrm{fg}$ & $90-45$ & 16 & 15 \\
\hline \multirow{2}{*}{$1 / 8^{\prime \prime}$} & $\mathrm{cf}-\mathrm{f}-\mathrm{cf}$ & $90-90$ & 28 & 19 \\
\cline { 2 - 5 } & $\mathrm{cf}-\mathrm{f}-\mathrm{cf}$ & $90-45$ & 33 & 22 \\
\cline { 2 - 5 } $\mathrm{F}$ & $\mathrm{cf}-\mathrm{f}-\mathrm{fg}$ & $90-90$ & 23 & 18 \\
\cline { 2 - 5 } A & $\mathrm{cf}-\mathrm{f}-\mathrm{fg}$ & $90-45$ & 19 & 16 \\
\cline { 2 - 5 } $\mathrm{cf}-\mathrm{f}-\mathrm{fg}$ & $45-90$ & 13 & 17 \\
\cline { 2 - 5 } M & $\mathrm{fg}-\mathrm{f}-\mathrm{fg}$ & $90-90$ & 18 & 14 \\
\cline { 2 - 5 } & $\mathrm{fg}-\mathrm{f}-\mathrm{fg}$ & $45-90$ & 15 & 14 \\
\hline
\end{tabular}

Table 5: Break test data comparing strength of 1/8" Divinicell foam to 1/16" balsa

The results of this testing show that, even when comparing balsa at its strongest, the Divinicell foam is stronger than the balsa by a small margin. As the foam maintains this strength in all directions rather than just one, the foam was chosen as the core material for all the composite layups of the aircraft (skin, hatches, winglets). A note on this break testing: there was a single data point for each test, and the testing itself was not entirely precise. As such, trends and relative values were used rather than exact values. Even with this (potentially large) error present, foam would still be used regardless of the true strength tolerances due to the increased rigidity (did not test deflection before failure, but foam was noticeably stiffer) of the foam for added impact resistance to the airframe and the strength remaining constant in all directions. 

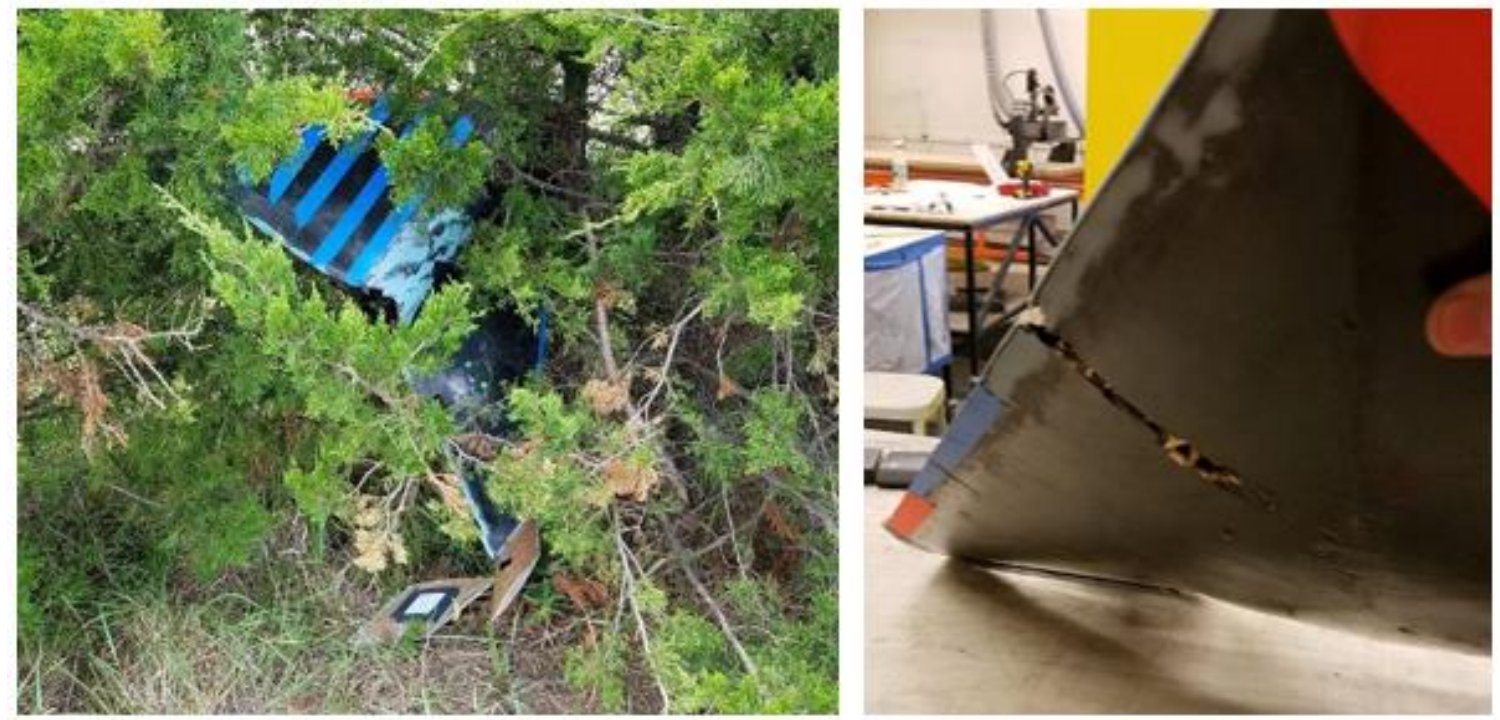

Figure 30: Damage to test aircraft from small trees and barbed wire fence at $70+m p h$ due to turbine failure

The two primary fabric options in consideration were $2 \mathrm{oz} / \mathrm{yd} \mathrm{d}^{2}$ carbon fiber and $3 \mathrm{oz} / \mathrm{yd}$ fiberglass. This weight of carbon fiber was used for the test aircraft and displayed a sufficient level of strength, as can be seen from a high-speed collision with a few trees and a barbed-wire fence. The skin gave with a clean tear until the barbed wire hit a piece of internal structure, as can be seen in Figure 30 above. Though the strength was favorable, the signal blocking/reflecting properties of the carbon fiber was not. Operating in an activity precipitating environment is expected to produce a reduction in signal transmission as it is, and this potential issue needs to be minimized. As such, the fiberglass was chosen for its RF transparency, while the layering was increased from the 1-core-1 layup on the test aircraft to a 2-core-1 layup on TIA to increase the strength of the skin even further.

A 3" strip of Kevlar $\left(4.6 \mathrm{oz} / \mathrm{yd}^{2}\right)$ was added along the centerline of the bottom skin. This is to help reduce punctures and other damage to this region, as this is where the primary damage on landing takes place. By having the Kevlar sheet, it will be more resistant to terrain features, 
increasing survivability should the aircraft have to land on gravel, asphalt, or other rough surfaces.

As for internal structure, the bulkheads were made of 5-layer aircraft plywood for its strength, light weight, and ease of manufacturing (CNC or laser cutting) with spar caps made of 1" wide strips of carbon tow placed in the layups of both the top and bottom skin. The bulkheads and all internal structure were first bonded to the top skin after it was manufactured. After the structure was bonded to the top skin and fully cured, the bottom skin was then bonded to the other side of the structure with extra epoxy placed all along the outer edges of both skins, giving a seal along all exterior edges from inside the airframe.

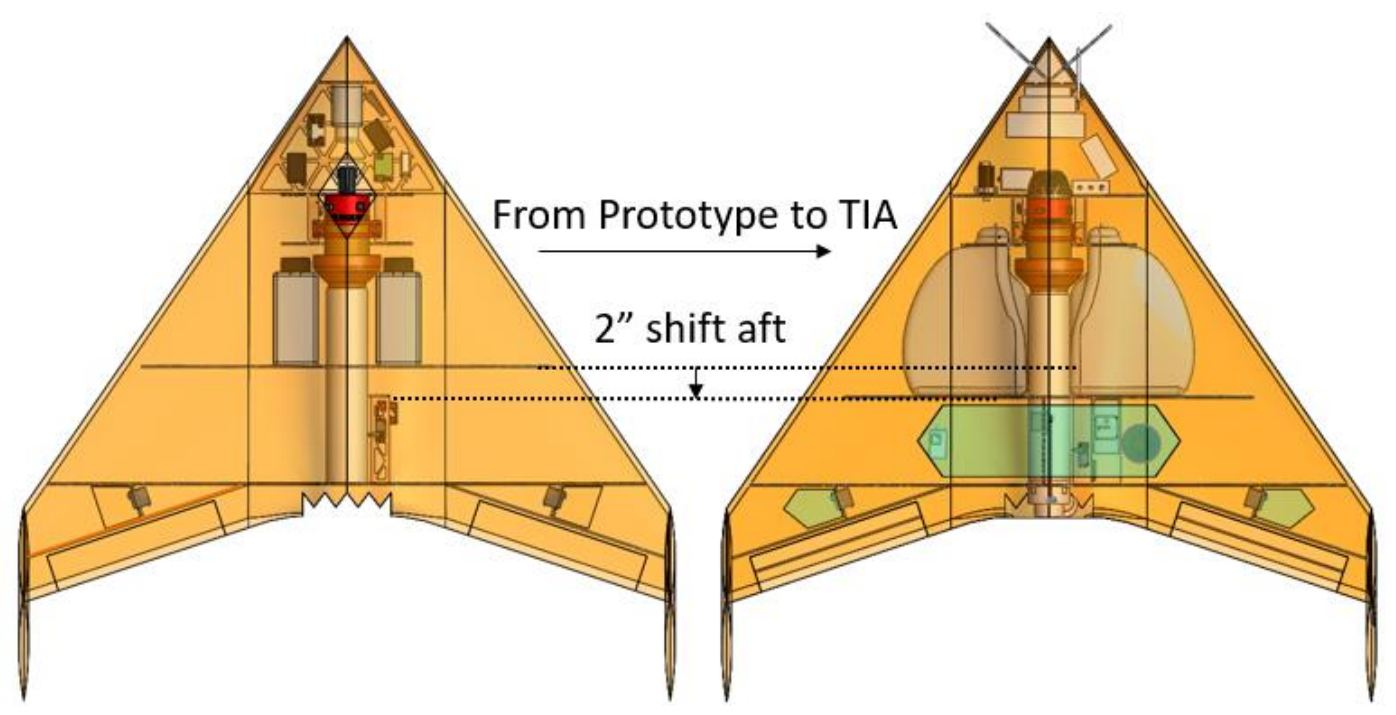

Figure 31: Mid bulkhead shift to accommodate larger fuel tanks

Since endurance and range are a large factor in this redesign, the fuel tanks became a major focus. To utilize as much internal volume as possible while remaining as close to the $\mathrm{CG}$ of the aircraft as possible, the Mid Bulkhead was moved backwards by 2 inches. The extra space allowed for large custom fuel tanks ( $60 \mathrm{fl}$ ounces each, $120 \mathrm{fl}$ ounces in total) to be designed, more than doubling the flight time of the aircraft even while using a turbine with a nearly $50 \%$ higher fuel 
burn rate $(5.46 \mathrm{oz} / \mathrm{min}$ vs $8.1 \mathrm{oz} / \mathrm{min})$. Doing so also removed a piece of redundant structure, as there the prototype aircraft had a vertical L-bracket to support the yaw thrust vectoring servo mount. The spar being pushed to this location absorbed this structural piece, allowing for the Mid Bullhead to bear that load directly.

\subsubsection{Hatch Manufacturing}

Prior to manufacturing a new, custom airframe, the hatches must first be created. This is due to the overall composite layup process, where the airframe skins are layed up on top of the hatches to ensure a smooth final surface transition from the exterior skin to the exterior of the hatches. These hatches were sized to allow sufficient operating room to install and replace components with relative ease. A short description explaining the sizing of each hatch is below.

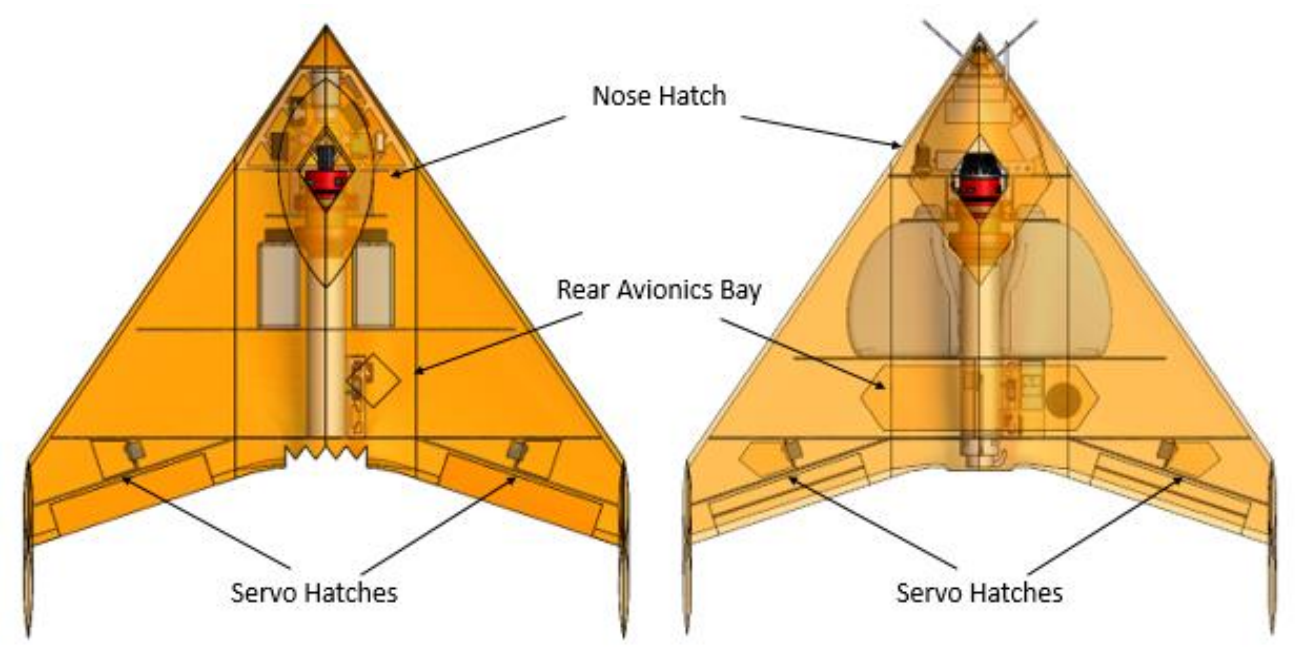

Figure 32: Hatch evolution from Diamondback to TIA

The nose hatch was generally sized to allow for full access to all components from the nose (FPV camera) back to the turbine. The hatch is wide enough to allow access to the embedded fuel tanks in the event of a leak or clunk replacement. The prototype aircraft's nose 
hatch left very little side wall for the nose, which was deemed insufficient for the intended use of TIA. As such, the hatch size was reduced (Figure 33) to allow for more structural rigidity in the nose.
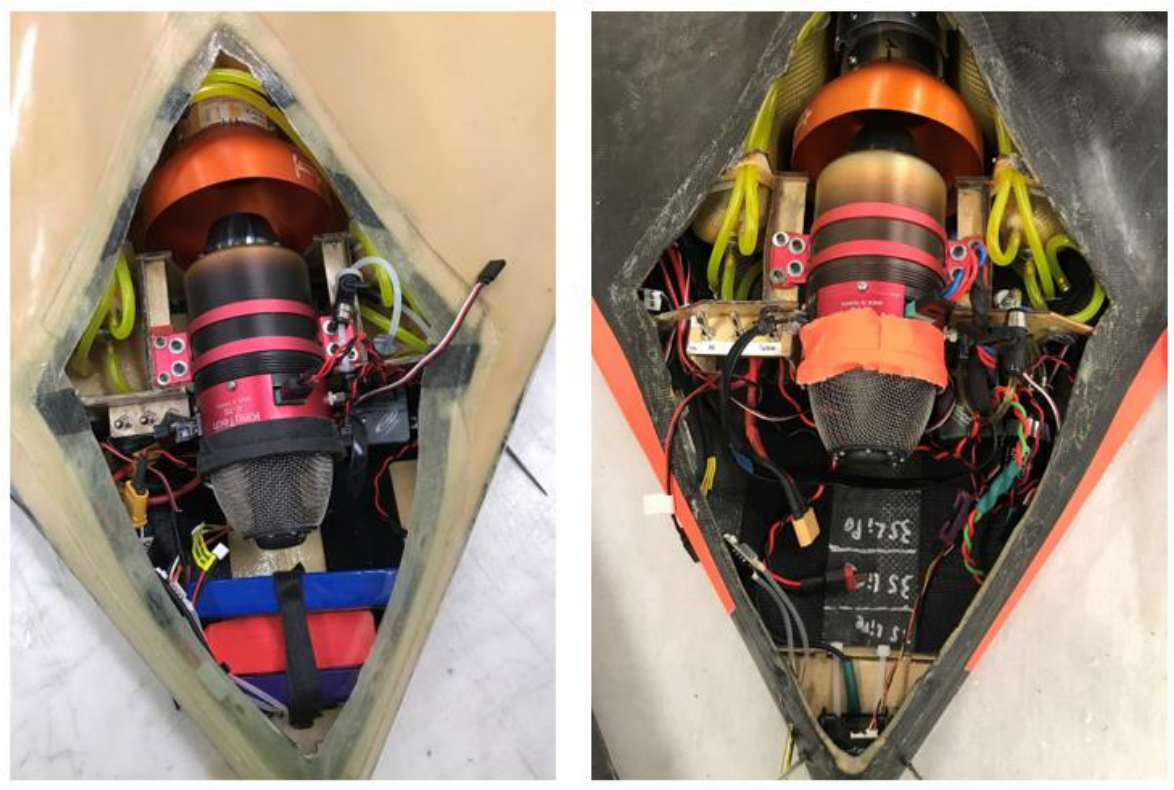

Figure 33: TIA (left) hatch cutaway area vs prototype (right)

The reasoning for the overall diamond shape of the hatch is to preserve strength of the skin, as any area occupied by the hatch results in a reduced structural efficiency, while ensuring the hatch can be properly secured during flight. The rear half of the hatch (area aft of the inlet hole) allows for extra mounting area to ensure the hatch remains secure through all flight profiles and gusts. To keep the nose hatch secured, magnets were included in the layups of both the skin and the hatch, as can be seen in Figure 34. This hatch, along with all other hatches, were also secured with screws that were secured with blind nuts epoxied to the inside of the airframes skin. 


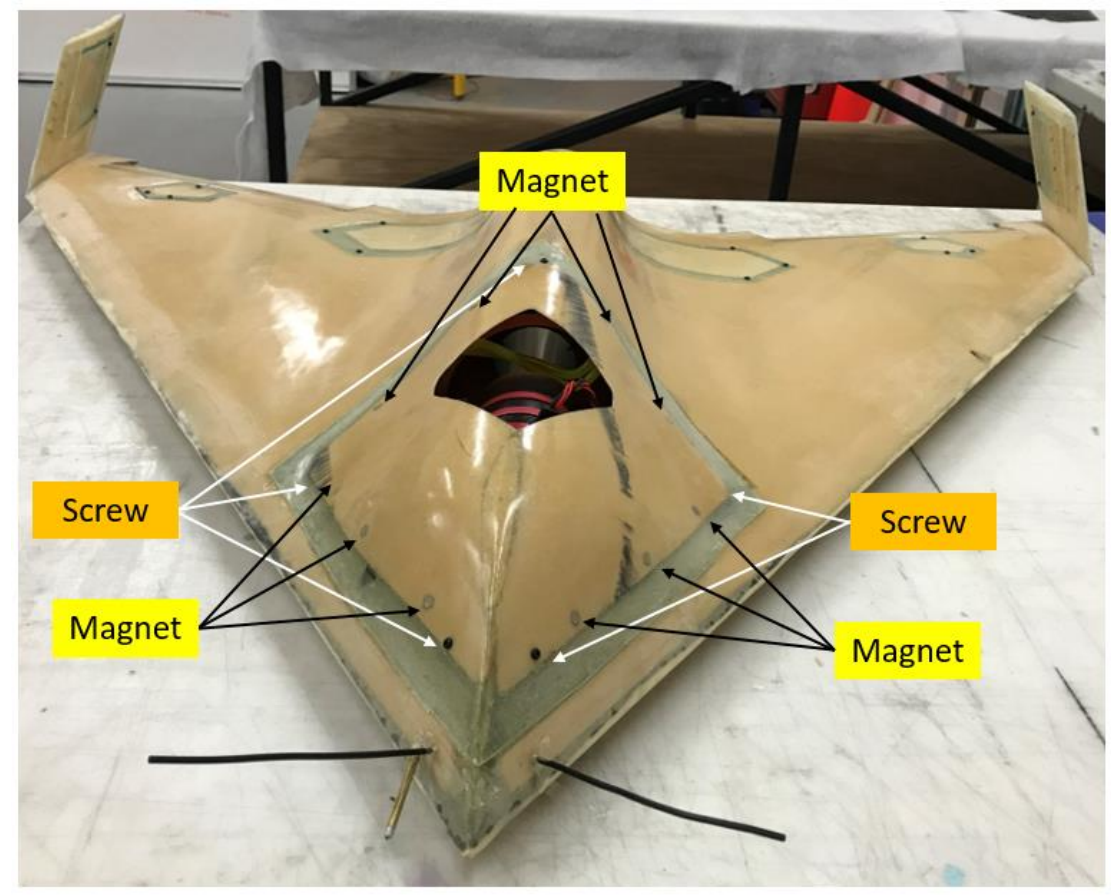

Figure 34: TIA nose hatch showing magnet and screw locations

The rear avionics hatch on the initial airframe was exclusively on the Starboard side to give access to the thrust vectoring servos and the rate gyro. This hatch was expanded to include the Port side as well for a payload bay. A future goal is to integrate a dropping mechanism with the aircraft in this bay, allowing for targeted observations of atmospheric system much like the happened in both project Torus and Tropical Cyclone Structure-2008 did with their dropsondes. This future expansion was included in the design. This hatch was also secured with screws around its perimeter.

The elevon hatches not only provide access to each control surface servo, but also to the wiring running into each winglet. The Port winglet houses the FPV video antenna and will house the atmospheric sensors once ready for in-situ measurement testing (future work), while the Starboard winglet houses the Pixhawk's long range antenna. These hatches must provide access to both install and remove the servos and the respective screws that secure the servo to the 
structure. Like the other hatches, these two are also secured using screws along the perimeter of the hatch.

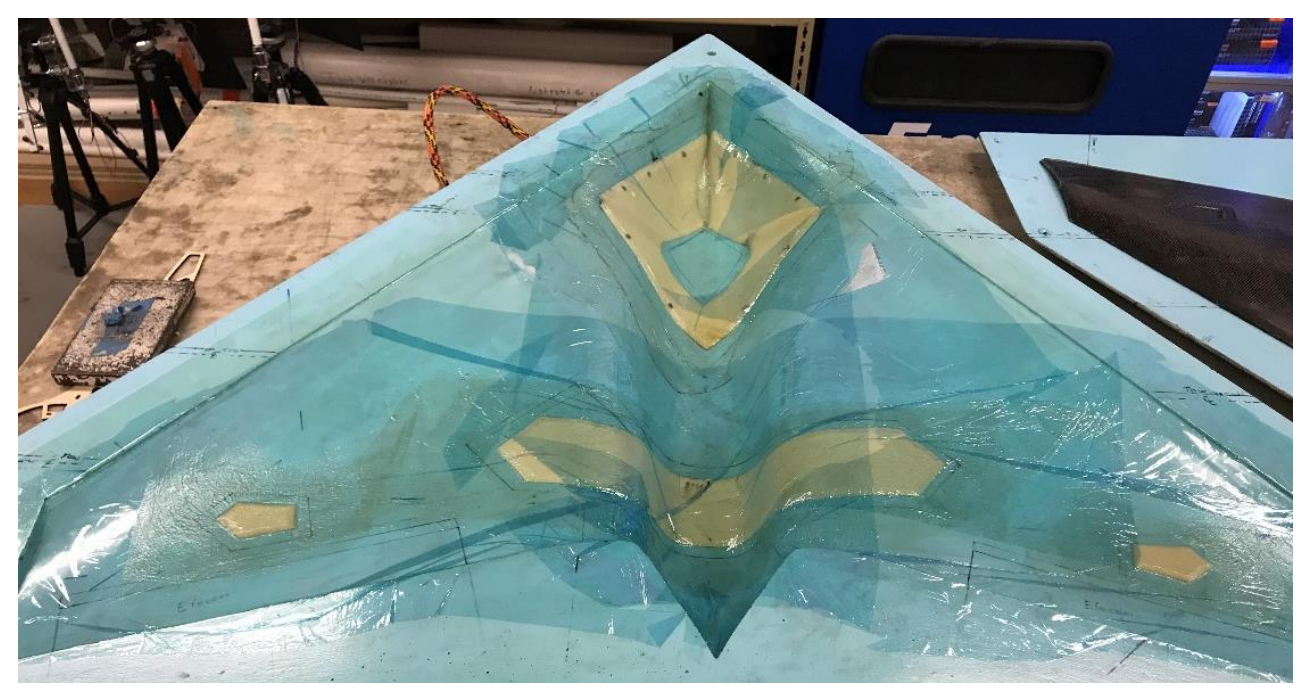

Figure 35: Hatches after layup but before removal from mold

All hatches utilized a 0.5 " flange over the actual opening to allow room to screw the hatches down without compromising the structural integrity of the hatch. To further assist this, in the layup of each hatch, a 0.75 " wide strip of tooling fiberglass $\left(10 \mathrm{oz} / \mathrm{yd}^{2}\right)$ was applied not only to ensure the screw shafts would not rip through the fiberglass flange when under loads, but also to give the edges of the flange extra rigidity to avoid any flutter they might experience in flight.

\subsubsection{Skin Manufacturing}

After the hatches were manufactured, the skin was next. To prepare for the skins, the hatches were removed for heat-forming of the foam to the mold. Once the foam was heat formed, the hatches we placed back on the molds with double sided tape to keep them stationary during the layup process. To prevent any epoxy from leaking under the hatch, modeling clay was placed 
around the edges of the hatches. The entire mold was then waxed and released as documented in Hunt's paper, even on top of the hatches and the clay.

The normal layup process then proceeded. The order of the layup was: 1 strip of 4" wide Kevlar (bottom skin only), 2 layers of $3 \mathrm{oz} / \mathrm{yd}^{2}, 1$ layer of 1/8" Divinicell foam, 1 more layer of the same fiberglass, then various lengths of carbon tow laterally on all bulkhead locations to act as spar caps as well as lengths longitudinally to increase the airframe's resistance to bending from nose to aft (important due to large hatch cutouts). The Kevlar was added to reduce damage to the crown along the bottom skin from landing on unimproved surfaces (dirt roads, grassy fields, etc.).

After both skins were cured, the internal structure was then added. The internal structure was placed in the top skin as it had the most complex geometry, which allows the structure to statically sit in this half in a more stable manner than on the bottom half. It is during this time that the fuel tanks are installed. They are installed during the layup due to their size, as a hatch allowing for their removal would not outweigh the reduction in structural efficiency the airframe would then have. Once the structure is confirmed to fit in the top half, the bottom half was then added on top to ensure a proper fit all around. Some components were sanded to fit as needed. 


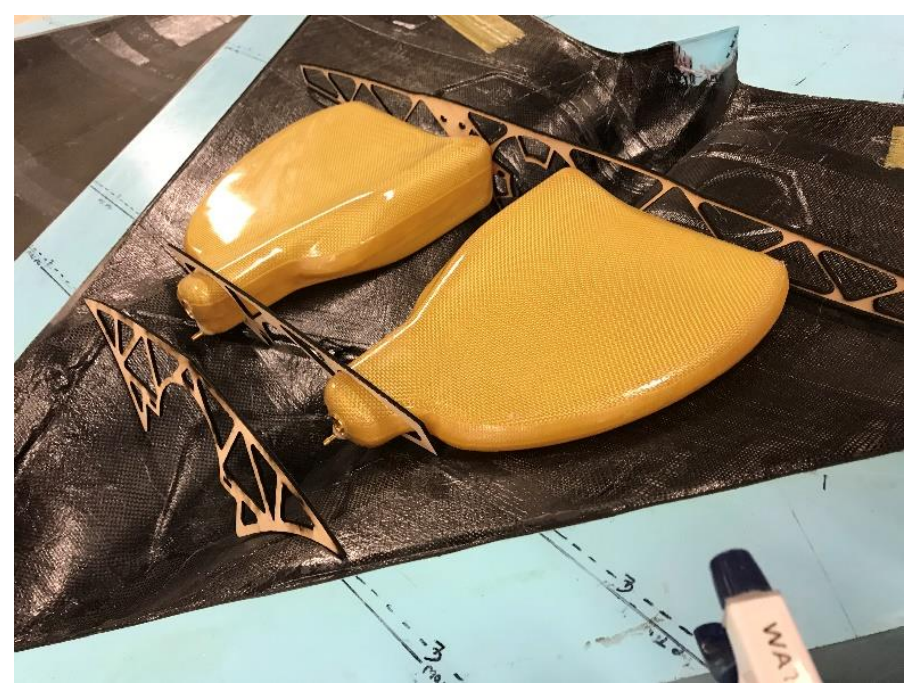

Figure 36: Test fitting the fuel tanks with the primary structure on a prototype airframe.

With the structure set and all sides fitting appropriately, the structure was epoxied to the top skin. After that batch cured the two halves were then epoxied together. This was done by applying a bead of epoxy along all structure being bonded to the bottom skin as well as along all external edges on both halves. When the halves are placed together, this creates a seal to bond to prevent the two halves from splitting. The halves are left in the molds, with the top skin resting in its mold on the table, with the bottom skin placed on top of the structure protruding from the top skin, with the bottom skins mold resting on top of the bottom skin. This allowed the weight of the bottom skin's mold to compress the composite pieces together, ensuring a strong bond.

To minimize the chance of the skins slowly splitting over many flights, a 2" wide strip of tooling fiberglass $\left(5 \mathrm{oz} / \mathrm{yd}^{2}\right)$ was epoxied over all exterior edges. After this leading edge tape had fully cured, it was then sanded down to be a smooth surface.

\subsubsection{Winglet Manufacturing}


The winglets were made much the same way as both the hatches and the skins were with the exception of an integrated hatch. Unlike the other hatches on the airframe that are removable, this hatch is fixed to the winglet itself by a Kevlar hinge. To create such a composite, a few extra steps are required.

Due to the how the layup process works, all hatches are made before any skins are created. As such, the process begins there. To give the most internal volume possible in the thin winglets, the hatch was made exclusively of three layers of fiberglass plus the Kevlar hinge. This process went as follows: two sheets of fiberglass were layed up in the mold, a 1" wide strip of Kevlar was added to the layup with half the Kevlar on the fiberglass along the hinge line while the other half is wrapped in a release plastic (prevents epoxy from soaking through under vacuum pressure), then the final layer of fiberglass as added on top, sandwiching half of the Kavlar. The part was then put under vacuum pressure as usual.

After the part finished curing, it was then trimmed to size according to the mold specifications. Instead of the clay process used for hatches in the airframe skins, this process involved releasing both sides of the hatch's fiberglass, taking special care to avoid any release getting on the Kevlar. Release prevents bonding of epoxy, which is great for keeping epoxy off the mold and other parts, but very detrimental when applied to a part wishing to be epoxied, especially a part as critical as the hinge line. The hatch was then secured to its location on the mold using double sided tape. At this point, the plastic release that has been over the Kevlar hinge was removed. If epoxy bled under the release paper (as can happened with the hatch in Figure 37), then simply scoring the epoxied Kevlar is sufficient. Generously coat the exposed Kevlar with epoxy, then the normal skin layup process can proceeded with one notable exception. 


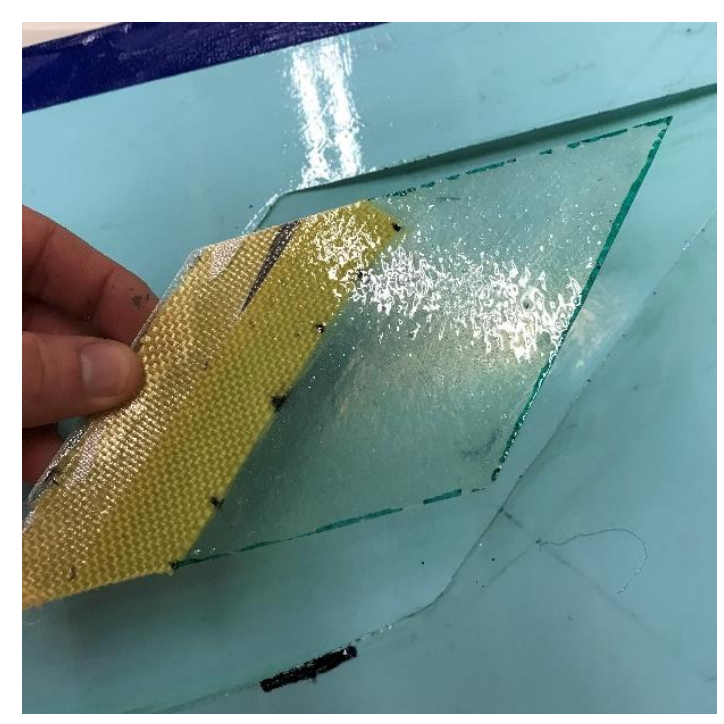

Figure 37: Winglet hatch post-cure and post-trim

Before the first layer of the 2-core-1 fiberglass layup is laid down, a 2" wide strip of preepoxied fiberglass is laid down under the Kevlar hinge with one side running along the hingeline established by the already-cured epoxy portion of the hatch and the rest protruding from under the Kevlar. This oversized fiberglass piece will bond to the Kevlar on the outside while also bonding to the other fiberglass layers, sealing the Kevlar in-between layers of fiberglass, giving an additional layer of strength. After this partial piece is laid down, the rest of the layup continues as normal, with both the outer layers laid down, followed by the Divinicell foam core, and a final layer of fiberglass.

Once this assembly is fully cured, the first thing is to careful break the residual epoxy that created a bond between the hatch and the inner skin of the winglet, preventing the hatch from opening. This is done with a plastic razor blade to prevent from cutting into the hatch or the winglet skin. With the hatch released, the hinge line must be scored and worked, just like control surfaces on composite UAS, in order to easily open and close. 
At this stage, the winglets are almost ready to be bonded together. Since the winglets are screwed in to the airframe, a simple structure of epoxy is added at these points to prevent the screw from ripping through the winglet when under a load.

With both halves of the winglets have a sufficient bounded area of epoxy to allow a screw to have purchase, the halves are ready to be bonded together. This process follows the same method as for the airframe, just without the added internal structure. Epoxy was placed along all exterior edges and the epoxy boxes, ensuring a positive seal.

After the halves are cured together, the immediate skin under the hatch was cut away, leaving a $1 / 4 "$ border all around to allow the hatch to be secured. Blind nuts were installed on the corners opposite the hinge corners to allow the hatch to be screwed down in the same way the other hatches on the aircraft are. Holes are then cut in the bottom of the winglet to allow wires to pass through from the winglets into the side of the airframe. Wood screws are then used to pin the winglets to the airframe on both the forward and aft sides of the winglet.

\section{$\underline{4.2 .5 \text { Component Installation }}$}

After the airframe was completed, all the equipment was then installed in their respective places. Though the majority of installed components don't necessitate a description, proper installation of the jet pipe and implementation of the fuel tubing, in particular the dual fuel filters, do.

The jet pipe, which is bifurcated to minimize the heat components in the rear of the airframe are subjected to, was purchased from Grumania Jets at an oversized dimension of $50 \mathrm{~cm}$ in length. This kind of jet pipe features an inner tube of steel to withstand the high exhaust gas temperatures of the turbine (can reach as high as $700^{\circ} \mathrm{C}$ ) with an outer tube of aluminum 
separated by a small gap to allow extra airflow to cool the inner steel and prevent the aluminum from overheating. Being oversized and two tubes overlaid on eachother meant a little extra work had to be done to ensure the pipe had a snug, proper fit.
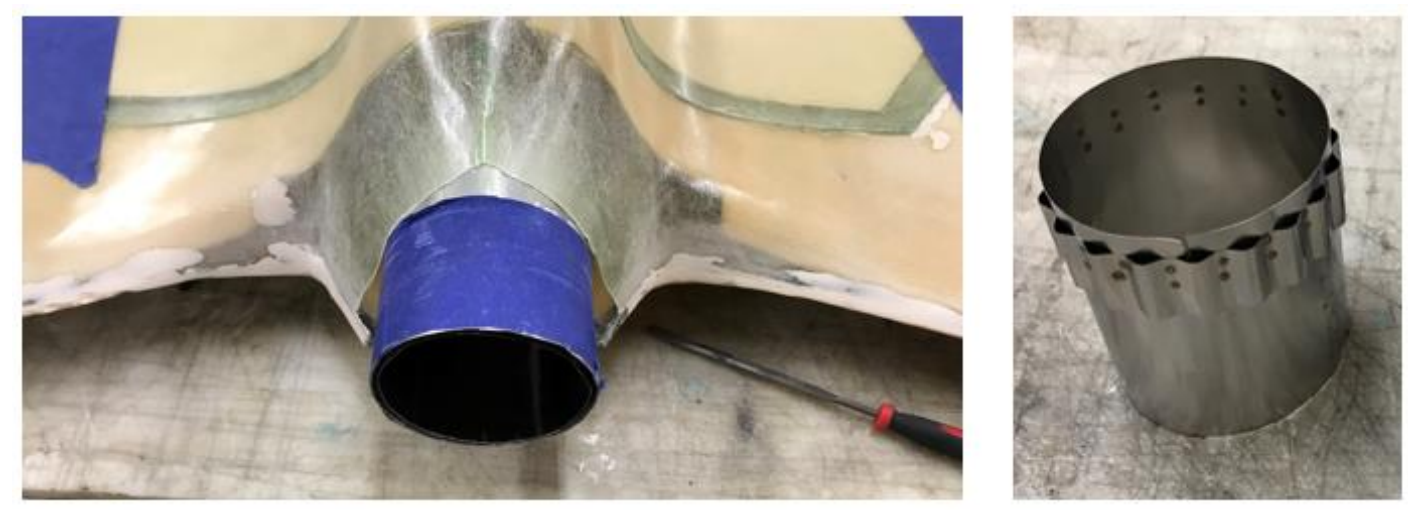

Figure 38: Sizing and trimming of the jet pipe

This was done by wrapping tape around the jet pipe at the line marked to trim it to, with a perfect overlap ensuring a circular path. The excess was then removed. As can be seen in Figure 38 , the inner steel pipe has an extra structure spot welded to it to maintain a constant separation between the inner and outer pipe. To produce a similar space, the thrust vectoring unit was riveted to the jet pipe. The rivet went through the collar of the thrust vectoring unit through the outer aluminum pipe, with the inner steel portion of the rivet resting against the inner steel pipe. Even after many flights and ground test, this configuration has yielded no issues.

The other primary installation consideration is the fuel tubing that links the dual fuel tanks to the fuel pump. Each tank has both a vent line and a fuel line. The process is simple: the vent line allows the turbine to draw in air to the fuel tank as the fuel line removes fuel. To ensure the fuel tanks do no leak fuel when handling it at max fuel, the vent line on one tank was looped over the bell mouth of the jet pipe and back down to the other fuel tank. This was done on both to ensure enough static pressure exists in the lines to help prevent unwanted fuel leakage. The 
location the vent lines were placed were shown to be sufficiently safe from the exhaust gases from repeated testing, as in no test were the lines in any danger of melting.

The fuel lines are a different story. To minimize the work required by the fuel pump, the lines were all installed in a co-planar orientation. This meant the fuel tubes were to be run underneath the turbine, which presents a major risk to the lines melting. To supplement this, the fuel lines were inserted through a heat-resistant tubing as well as secured to the bottom skin of the aircraft in two places. This not only helps to prevent the tubing from touching the turbine, the shielding ensures that even if that were to happen, the tubing would not be harmed.

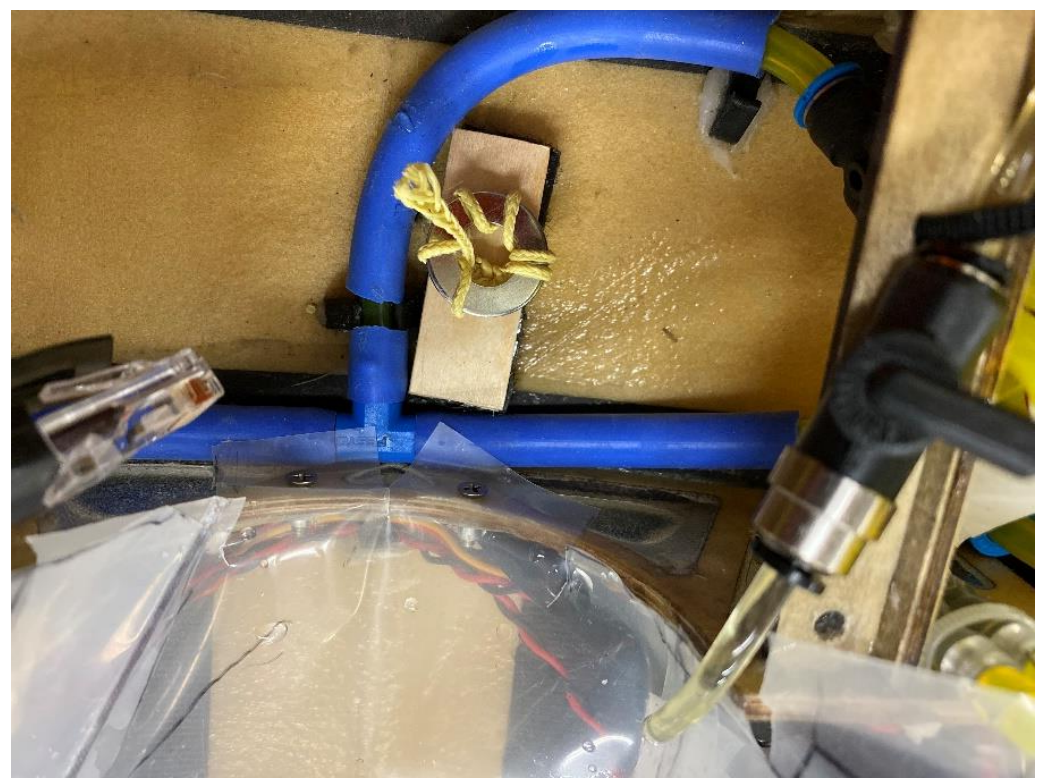

Figure 39: Shielded fuel tubing under turbine and secured to skin. Also visible is the launcher attachment line with nose Mylar screw locations.

Both the fuel tanks had equivalent lengths of tubing meet at a T-fitting directly under the turbine, with a singlet length then passing the fuel to a Y-fitting. This Y-fitting allowed for fuel to be added to the tanks through one of the upper Y-branches, while the other two Y-branches acted as a straight-line bypass to allow fuel to be sent to the fuel pump. The fuel tubing was covered in a flame and heat resistant rubber tubing, preventing the fuel tubing from melting during flight. To 
ensure the tubing remains secured to the skin, gripping supports were epoxied to the skin to ensure the tubing is held secure. Typically, the only place a jet has fuel filter is between the fuel pump and the turbine fuel inlet. However, due to a fuel pump failure that arose from a small epoxy particle dislodging from the custom composite fuel tanks, a fuel filter was installed before the fuel pump. This means the jet now has two fuel filters, both aligned vertically to prevent the buildup of air bubbles that could potentially cause the turbine to flame out, or shut down due to insufficient fuel flow.
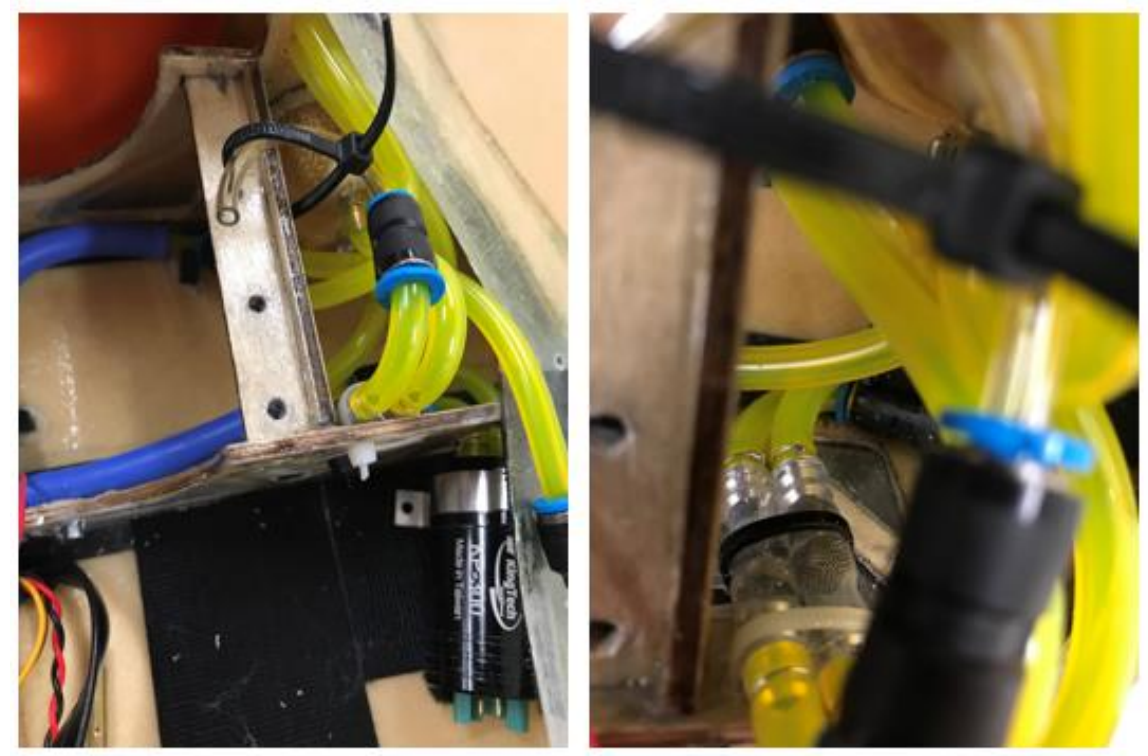

Figure 40: Dual transparent fuel filters installed vertically

In this configuration, the fuel filters can be routinely inspected for any debris accumulation. In theory, there should never be any, but this gives a level of redundant security to ensure the turbine has minimal chances of being shut down mid-flight.

All other systems are straight forward to install and do not warrant explanations. 
As this aircraft will likely be operating in an actively precipitating environment, much care was taken to ensure minimal water accumulated inside the airframe, namely near the sensitive avionics. This was implemented in various ways, starting in the nose, around each of the hatches, and around the rear avionics bay.

The nose of the aircraft not only holds the most critical avionics and propulsive components (RC receiver, batteries, turbine, ECU, etc.), but it also is the hardest to secure against water intrusion as the air-breathing turbine requires an open hole through which to draw air. This hole also allows for water from rain to enter the airframe as well. To repel the water, a sheet of Mylar was used to ensure water does not splash or drip down into the nose avionics bay. This sheet of Mylar was cut to size, then using a thermal contact machine, three individual pieces were fused together. The newly fused Mylar piece was taped under the nose hatch, which is screwed down, and the lower section of the Mylar is screwed into the bottom of the Front Engine Bulkhead. A test was done to see if the turbine would melt the Mylar, but ground testing revealed it has sufficient heat resistance. Even after a full flight with the Mylar installed there were no issues with overheating.
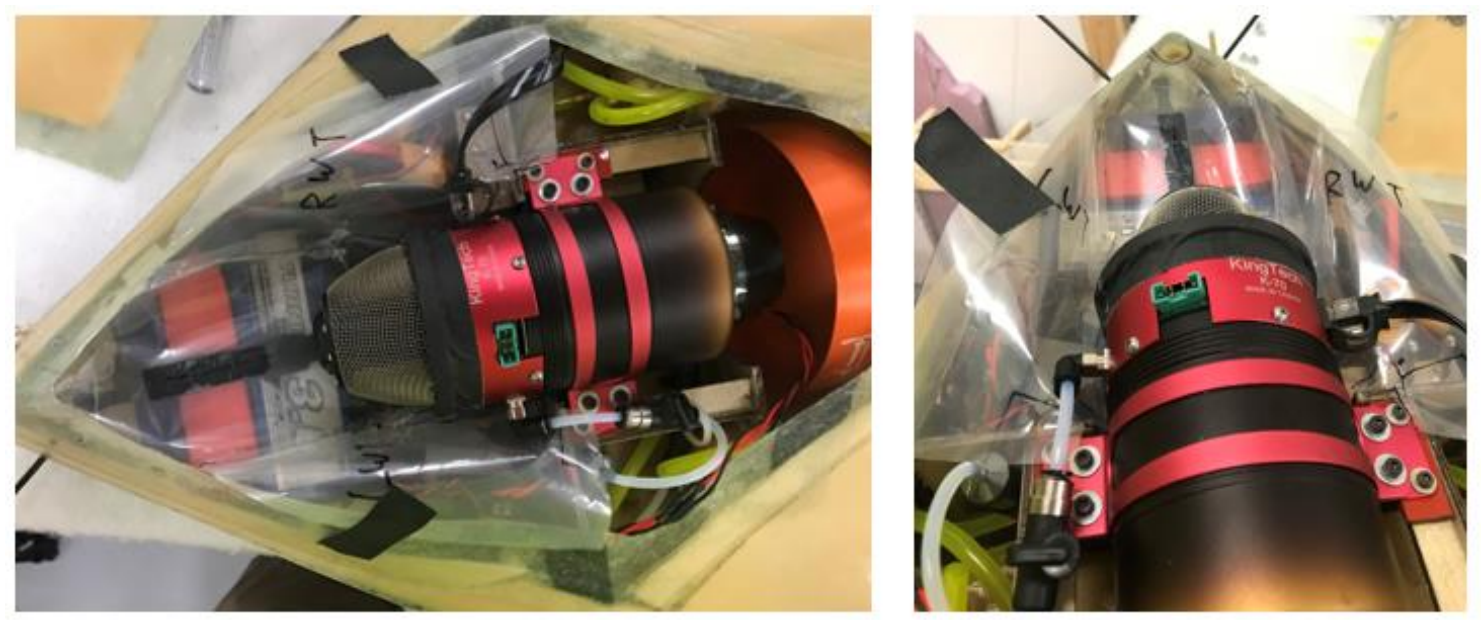

Figure 41: Mylar shielding in the nose of the airframe 
This same type of Mylar was also installed as a shield in the Rear Avionics Bay, preventing any splash from the front of the aircraft reaching the Pixhawk, GPS, or Aura 8 systems. The servos are all water-resistant, so they are expected to survive any potential splash they might receive from water that bypasses the jet exhaust into the jet pipe. However, to ensure that water doesn't come pouring through the edges of the rear hatches, shower tube sealant was used to create a gasket along the outer edge of all hatches.

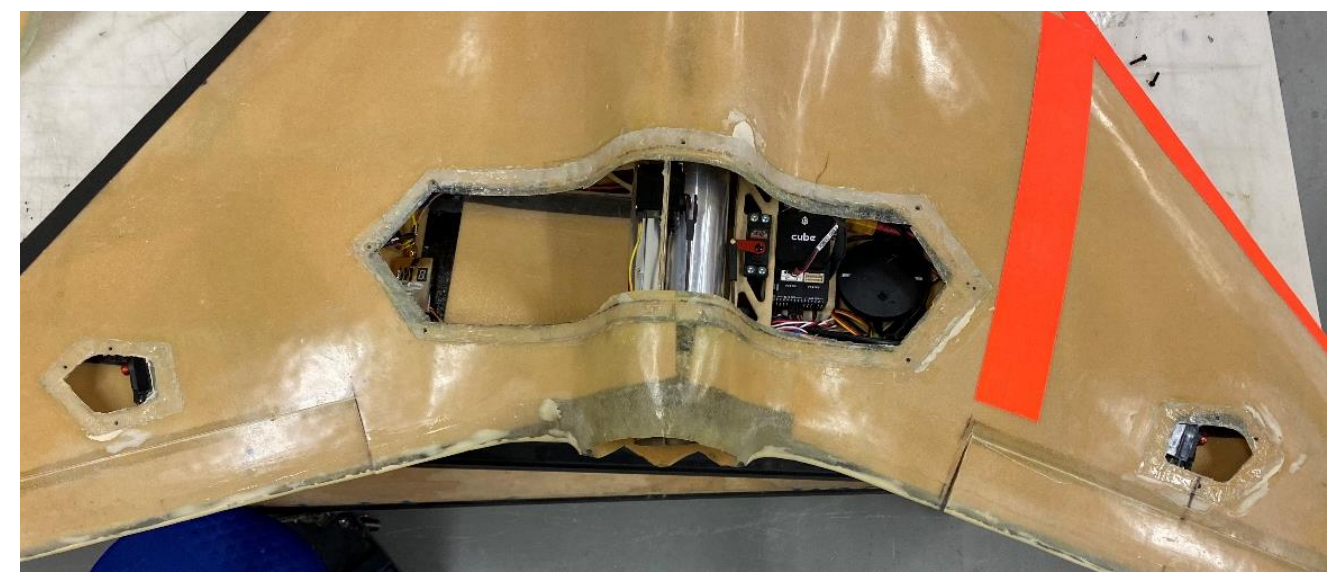

Figure 42: Shower tub sealant gasket along all hatches

Through these different methods, it is assumed that the aircraft is sufficiently shielded from water ingestion in actively precipitating environments.

\subsubsection{Integrated Ground Testing}

With all systems installed, the final test before flying was to do a full systems ground test. Since the major issues were diagnosed and resolved on the prototype aircraft, the integrated ground testing proved a relatively simple endeavor. The first test was to ensure the Pixhawk was communicating properly with the GCS computer, and by changing the NetID (what specific frequency on a channel the systems communicate on), both were communicating in a matter of 
seconds. Next was the RC link. As the DragonLink RX and TX had already been linked for previous testing, that was also done with no issues. FPV video was also installed and started up with no issues.

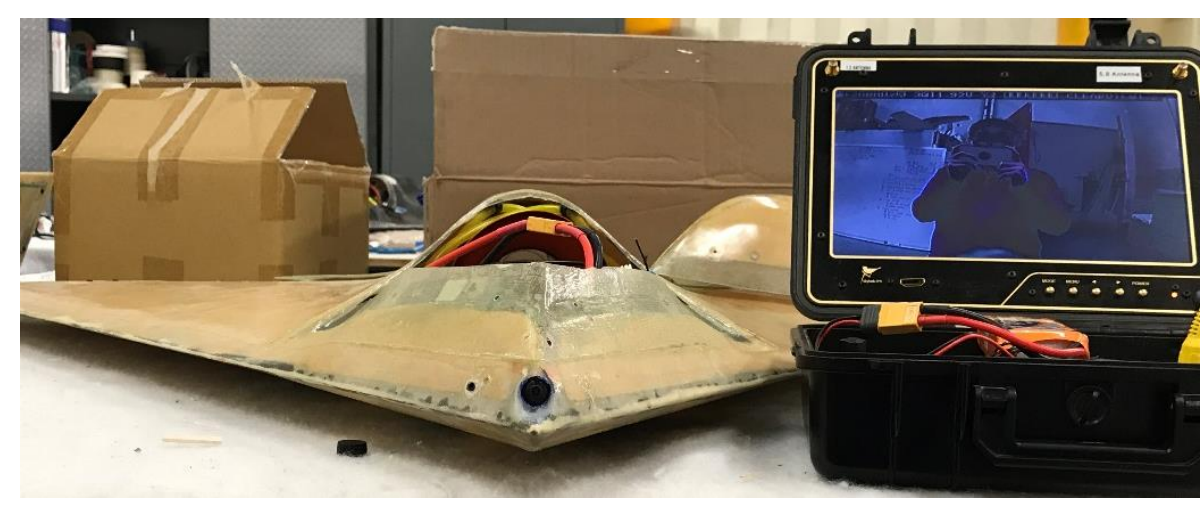

Figure 43: Testing of FPV video system

The only remaining portion to be tested was a live fire of the turbine with all systems installed. As such, the aircraft was fueled up and fired up on an outdoor testing platform. The full testing went as expected, with the turbine achieving full range of throttle with appropriate throttle response times, confirming that the testing on the prototype airframe had sufficiently prepared the final product for operation. There were many errors that were encountered, some worse than others, and a full resolution of said errors is found in Section 4.1.1.

\subsection{Launcher Build and Testing}

This section describes the build process of the launcher. It starts with the build of the initial design and how each leg of flight testing forced the modification of the design in various ways, starting with a lightweight single prop foamy to a larger, dual prop foamy, to the EDF prototype variant of TIA. 


\subsection{1: Launcher Build Process}

With the design finished and parts fully spec'd out, the next step was to turn the paper design into a physical one. The build process was straightforward: the main legs each had a pivot at the bottom to attach to the roof rack and T-plate on top for the wing supports with a T-Plate in the middle of the legs for the crossbar; each rear leg had pivots on both ends, one to attach to the roof rack and one to attach to the back of the main legs; two corner brackets off one of the main legs to support the weather station; and a single crossbeam support for the weather station boom attached using two pivots, one near the end of the boom and the other near the middle of the main crossbar. Each used the same M6 screws, with dual and single T-nut plates being used in their respective locations.

The biggest integration challenge was the linear actuator. It had to be mounted in such a way to prevent a large side load from acting on the actuator's motor (failure mode) while still allowing for repeated use with a simple reload method. The side load challenge was overcome by using a small excess piece of 8020 for the actuator's arm to rest on during operation. The other end of the actuator can freely pivot around a pin as part of the "standard mount". This mount came with the actuator, but had to be modified to fit the launcher. Two holes were drilled out of the metal for screws to pass through for mounting, and two more were placed in the same location on the opposite side to allow for access to the screws using a hex wrench. This allowed for the actuator's mount to be secured to the main crossbar, with a pin in this mount holding the actuator and allowing it to pivot. When the tether is loaded on the actuator's arm, the pivot will remain inline with the crossbar by resting on the small 8020 piece. On initial testing, the pivot was allowed to hang down when the tether was released. To prevent unnecessary side load on the actuator's 
mount, a large zip-tie was placed around the actuator giving it support when the tether was released.
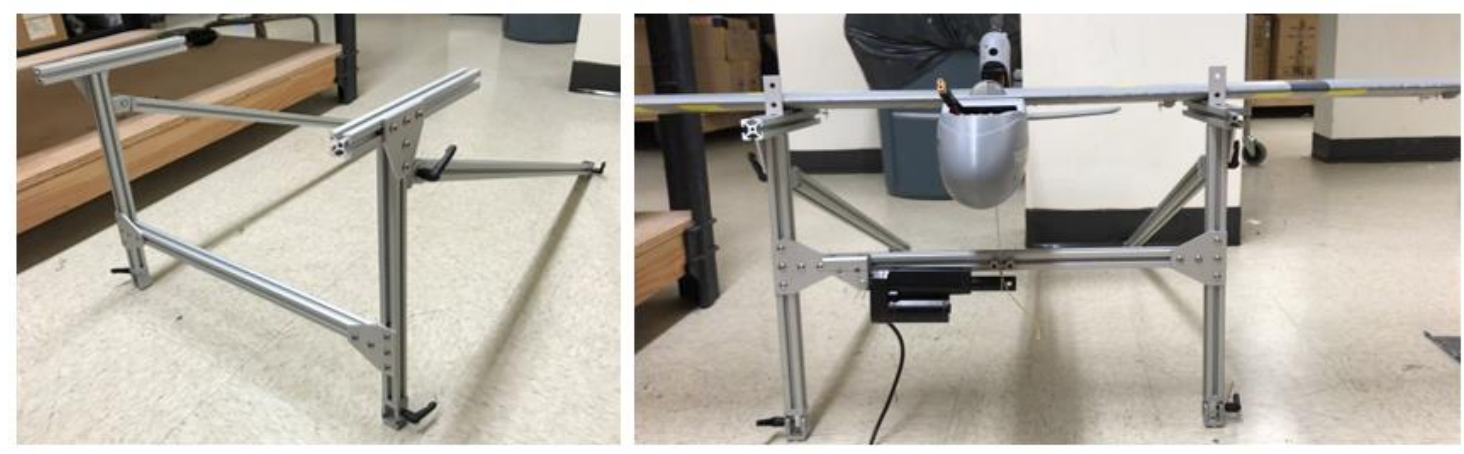

Figure 44: Initial build of launcher

While the overall concept of the build remained unchanged through the assembly process, there were certain considerations that did evolve as the launcher developed into a physical model. Namely of these is the vertical supports. The final design (D4) utilized a "flat top" wing support system with moveable vertical supports. The initial idea for this was to use an 8020 corner bracket on either side. After seeing the system in its physical form, that height $(<2$ inches) appeared too short, so 6 inch supports were cut to attach to the brackets should they prove to insufficient. Extra pieces for the fuselage support were also trimmed in the event they need to be exchanged. With the entire system being made of 8020 , interchanging parts proved to be easy as each component released with the loosening of 2-3 screws.

\subsection{2: Testing of Launching System}

\subsubsection{1: Testing Stability of System}


Once the launcher was assembled, it was installed on the launch vehicle and began undergoing a series of stability and rigidity checks. The first was to shake the launcher, which was done by standing on the side of the vehicle, grabbing the launcher, and giving it an easy shake, slowly making the motions more and more intense. The vehicle began to shake but the launcher did not slip or give at all. This signified that the launcher was ready for a driving test.

A GoPro was mounted on the weather system boom, and the vehicle slowly began speeding up on an empty road. The boom began a very minor sway at $50 \mathrm{mph}$, but was able to reach $80 \mathrm{mph}$ with no structural problems. This led to the use of a crossbar support for the boom, which took the form of an 8020 bar that attaches near the end of the boom on one end and in the middle of the main crossbar at the other. Another drive test confirmed the sway was effectively mitigated. With the launcher structurally capable of withstanding its operating environment, the process was moved to flight testing of a test airframe.

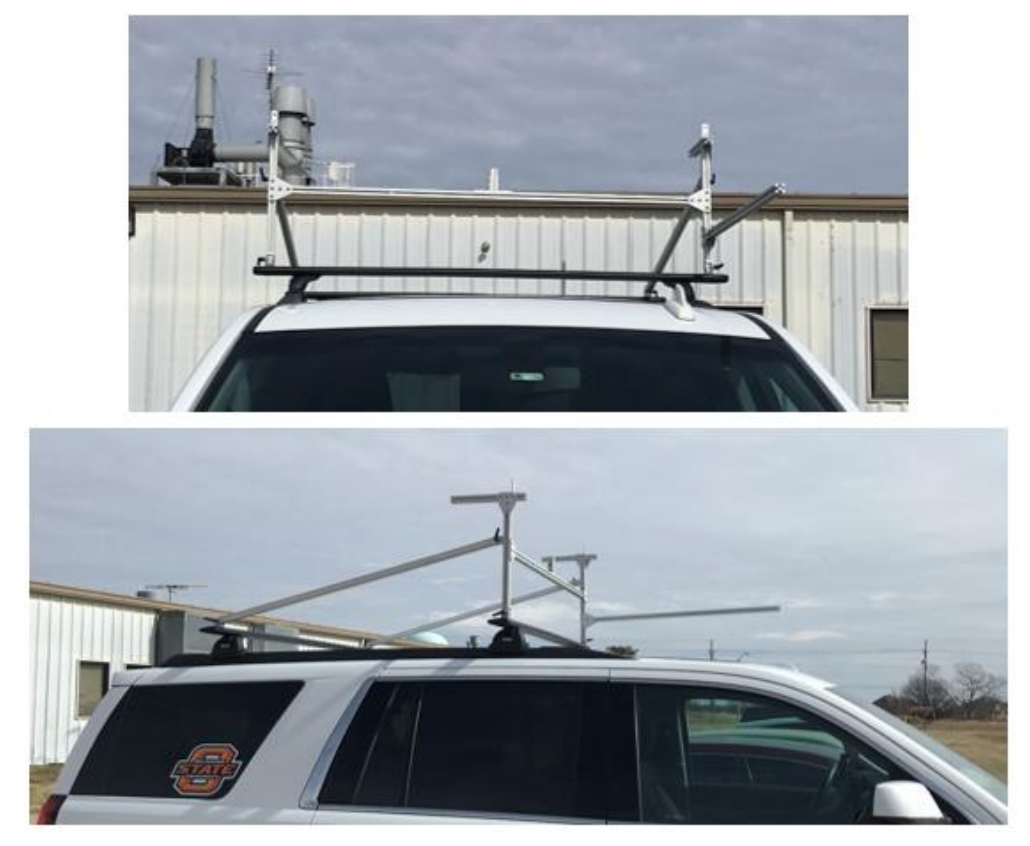

Figure 45: Launcher mounted on the launch vehicle

\subsubsection{2: Flight Test Plan}


The flight test plan as a whole is a procedural increase in aircraft weight and cost, allowing for modifications to be set in place and verified with the cheaper airframes before subjecting the expensive and sensor-laden aircraft to a launch. The procedure is: start with a semidisposable lightweight single prop aircraft (Firstar V2); advance to a heavier, more applicable single prop airframe (Skyhunter/Anaconda); move to a dual-prop foam aircraft (Believer); delta wing foam aircraft (Opterra); Diamondback EDF test frame (“Beast”); Diamondback jet test frame (ProtoRAE/TIA); then finishing with the full TIA. These aircraft were chosen based on availability and convenience as well as to demonstrate the wide range of aircraft types that can successfully be launcher.

For the first flight test, an airframe was chosen that would be very easy to replace should the launch fail for any reason. This is one of the primary reason that the Firstar V2 was chosen, as a new system costs just $\$ 140$ for the entire airframe and avionics. It is a lightweight aircraft $(\sim 3$ pounds TO) that should demonstrate the launch condition easily, as the lightweight translates to a slower speed prior to release for the launch vehicle. The Firstar V2 also has a long wingspan (77"), which is similar to what other heavier aircraft that will later be launched from this system utilize. The airframe is made of a blow-molded Nylon plastic with the collapsible single propeller located above the fuselage, making it very durable should the launch fail.

Each of the subsequent aircraft were chosen because they either were a low-cost step towards launching the objective aircraft (Firstar, Opterra, "Beast") or because they were directly intended to be launched by this system (Skyhunter, Anaconda, Believer, TIA). Other custom aircraft are being considered for use by this system, but each of those aircraft are styled similar to one of the test aircraft, so they will not be reviewed in this paper. Should all of these aircraft demonstrate successful launches, any platform of fixed-wing sUAS (single prop, dual prop, 
turbojet, delta wing, tube-and-wing, etc.) will have been shown to successfully be deployed by this launching system.

Of note: the following section will describe each flight test sequence (FT). Though they will be read as though a single flight was conducted, each of the tests were conducted multiple times for repeatability. Most tests in a sequence were nearly spot-on replicas, but any notable deviations are noted as are the follow on flights to verify the source of the deviation.

\subsubsection{3: Flight Test 1: Firstar V2, Single Prop, Conventional Airframe}

As mentioned above, the maiden Flight Test (FT1) was accomplished using the Firstar V2. The weather station was mounted on the boom to give a sense of wind direction during the takeoff sequence, but testing determined this system alone would prove to be insufficient. [This will be one focus of future research: to develop a weather station that gives rapid updates $(\sim 1-2$ second) on ambient conditions.] The aircraft was secured to the launcher as initially intended: with the tether wrapping over the fuselage support down to the actuator and the wings resting against the corner bracket vertical supports. The launch vehicle was then driven up to launch speed $(20 \mathrm{mph})$ and the aircraft was released. 


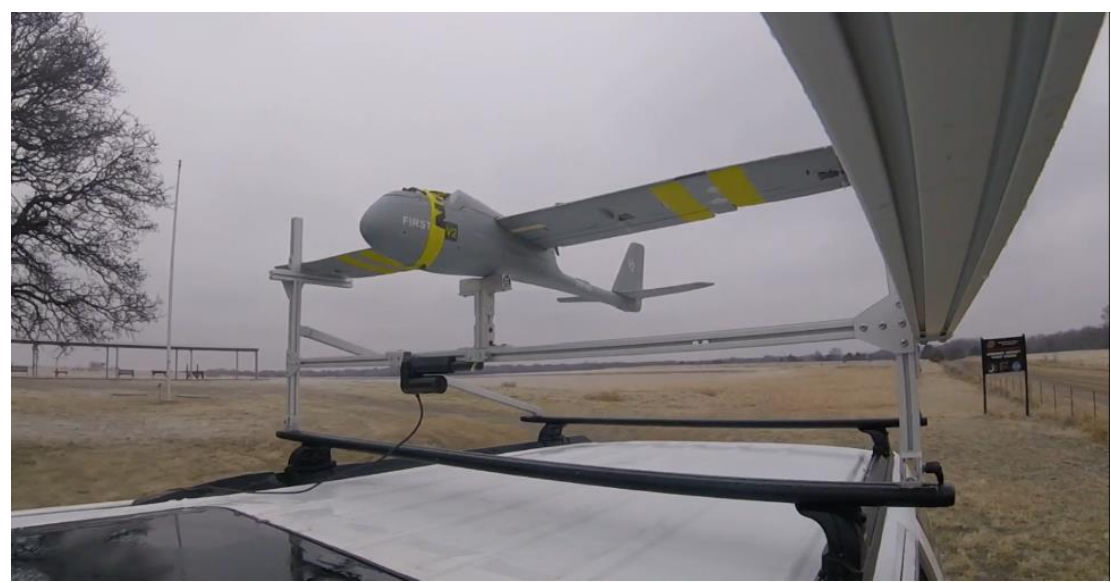

Figure 46: Firstar V2 on launcher before first launch attempt

Though the aircraft released successfully, review of video showed several areas for improvement. The most notable was the wings were bouncing around as the vehicle got up to speed, to the point that at one instance, a wing actually bounced over the vertical support before being balanced by the other wing pinned against the opposite vertical. Though the tether line was set with minimal slack, video review revealed the bending of the tether across the fuselage support to be the source of the slack.

Tethered

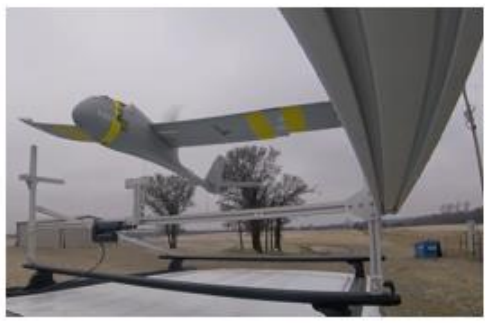

Release

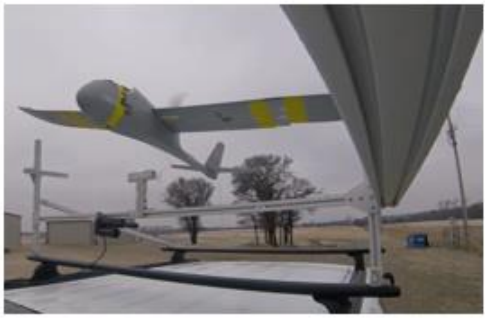

\section{Climb Out}

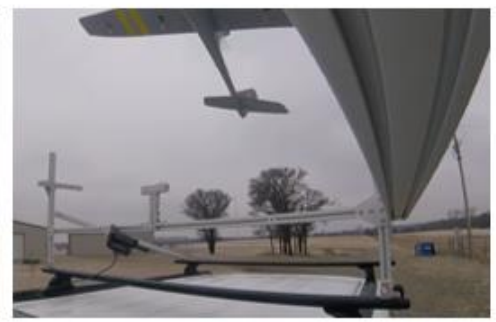

Figure 47: Maiden launch of Firstar V2

To circumvent this, the single bar of 8020 that was being used for the fuselage support was moved forward by placing a longitudinal 8020 support. All previous iterations relied on the fuselage mounting location to be in the same location as the linear actuator. This setup, by nature, was not dampening out the bouncing from the pre-launch conditions. By moving the fuselage 
support forward as a pseudo "nose support", as lift was generated by the wing, there would be a nose-down pitching moment, which meant the frame would be pressing down on the support structure, reducing the bouncing oscillations. This also permitted the tether to be completely taught with no room for excess to appear during the takeoff sequence by launcher geometry alone. With these changes in effect, the Firstar was again launched.

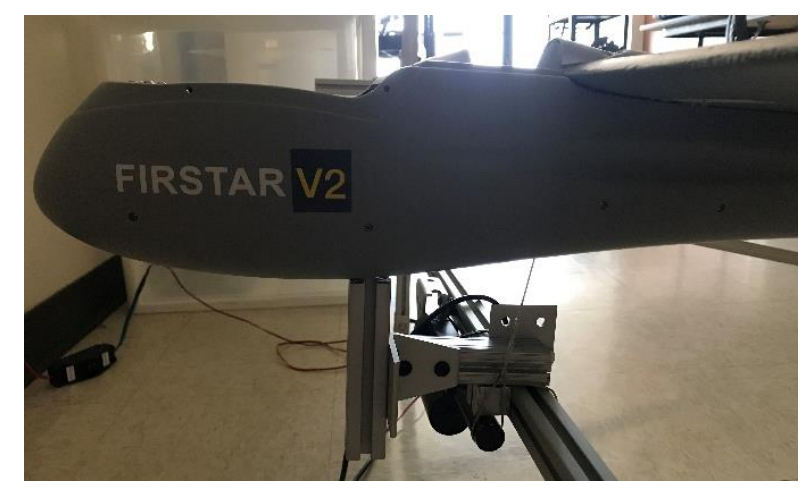

Figure 48: Launcher with nose support, Firstar V2

The aircraft and launch vehicle were again brought up to $20 \mathrm{mph}$ before releasing. This time, however, there was no noticeable wing bouncing and the Firstar did not move prior to being released. This model proved to be the most successful yet, with much of that success brought by the moving of the fuselage support forward of the dynamic points of interest of the aircraft, namely the Center of Gravity (CG), Aerodynamic Center (AC), and the Tether Point (TP). In the previous iterations, as lift was generated, there was a slight nose up pitching moment, which induced oscillations due to being tethered, and that coupled with the slack in the tether, permitted the wings to bounce over the vertical supports. These have been corrected for, allowing the aircraft to remain very stable and unmoving prior to its release. With the primary drawbacks circumvented, the launcher moved on to the next stage of testing.

\subsubsection{4: Flight Test 2: Skyhunter, Single Prop, Conventional Airframe}


The second group of flight testing was conducted using a Skyhunter with $1800 \mathrm{~mm}$ wingspan. Though there a couple shared characteristics (namely traditional tube and wing, pusher electric prop), they do vary some. The Firstar is a lightweight molded plastic body with foam wing, whereas the Skyhunter uses EPO foam for skin on body and wings with carbon rods for the spar. The Firstar setup used in FT1 was a pure manual flight with no in-flight assistance (gryo, accelerometer, etc.), while the Skyhunter was used tested and flown with a Pixhawk 2.1 autopilot onboard, which allows for testing of auto takeoffs from the launcher. The Skyhunter was also chosen as heavier test aircraft, as it weighs closer to 8 pounds (depending on configuration) compared to the Firstar's 3 pounds. These differences, coupled with the reliability of the Skyhunter as a whole (workhorse of Oklahoma State's USRI), made the Skyhunter a logical intermediate step.

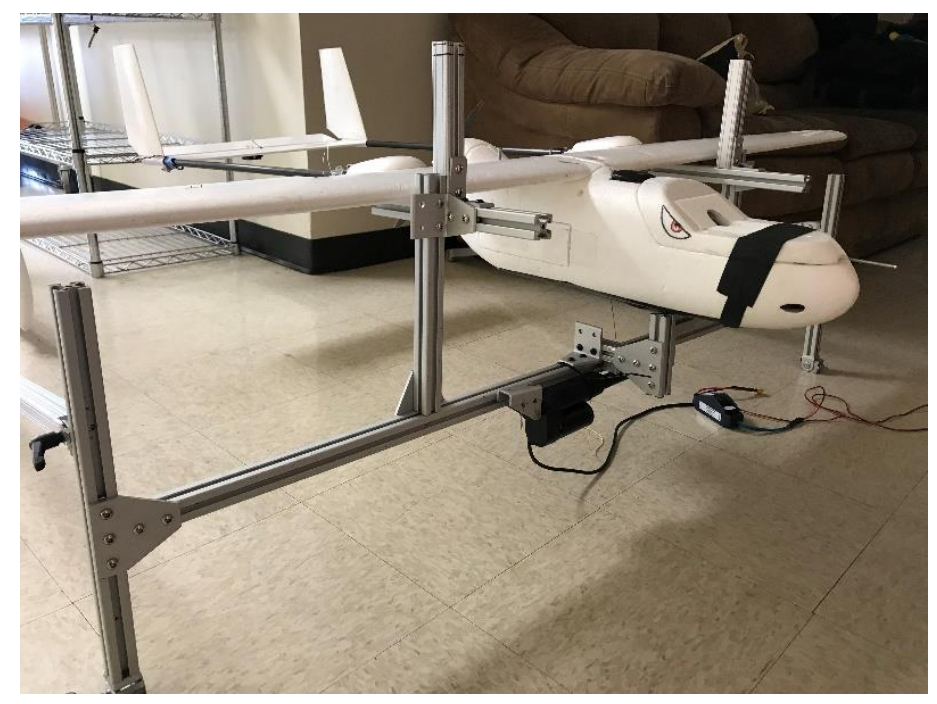

Figure 49: Skyhunter mounted on launcher

The initial flight of the Skyhunter (FT2) was done in full manual. The Pixhawk was installed, but all auto functions were bypassed, giving the pilot full control with no assistance to match the Firstar's flights. The overall launch sequence was the same for the Skyhunter as it was for the Firstar: Aircraft was readied and armed on the launch vehicle, pilot was stationed down 
the road in clear view of the system, vehicle was brought to launch speed (20 mph), driver honked to signal readiness, pilot activated full throttle, passenger confirmed, passenger activated release before reaching pilot and watched aircraft takeoff.

FT2 was a success, as the Skyhunter was launched with no issue. Since this was the first launch of a new aircraft, the pilot used full elevator deflection, which was shown to be a little too aggressive as the aircraft nearly stalled from the extreme angle of attack achieved. Future flights would be done with half deflection as a maximum, as the structural integrity of the aircraft must be considered.

For the next test set, flights were conducted with Fly-By-Wire A (FBWA) enabled. FBWA uses the Pixhawk's sensors to limit deflections and de-couple roll and pitch control inputs, allowing for easier pure roll or pure pitch commands. FBWA also uses gyros to help remove the influence of turbulence, varying wind speeds, and other unsteady features of the aircraft, allowing for smoother flights without requiring pilot input. This was tested as a launch condition as a precursor to full autopilot control on launch and to ensure launches would still be viable in high winds, which is a flight condition FBWA is often used. Having this kind of additional control augmentation allows the pilot to focus on the desired aircraft flight path while the autopilot handles the environmental conditions, allowing for even inexperienced pilots to conduct the takeoff.

The launch for this test was done at half of the maximum pitch deflection, as learned from the previous flights, and it was a much less aggressive launch. The aircraft climbed out at roughly $10^{\circ}-15^{\circ}$ angle of attack, which was deemed an appropriate climb out angle for this aircraft. The pilot was able to ensure an easier launch as he only had to give flight direction rather than fight the $7 \mathrm{mph}$ wind coming at $45^{\circ}$ from the front of the vehicles launch direction. The 
FBWA mode allowed for some drift due to this wind, but the pilot was able to easily correct for that by applying a little extra roll input. FBWA handled the rest of the flight control.

The final set of flights conducted with the Skyhunter was conducted in full autopilot mode. This has been the goal of flying with this aircraft, as it will enable testing of full autonomous control of the launch. Currently, this aircraft and many others are already capable of full mission pathing and control through the Pixhawk. Integrating the launch sequence into the auto-mission would make the flight profile such that the pilot only monitors the aircraft until landing, which further decreases the required skill level of the pilot.

The mission of this flight was such that the autopilot armed the motor once a certain GPS ground speed was reached (20 mph), aircraft would climb out at a $12^{\circ}$ angle of attack, level off at $150 \mathrm{ft}$ AGL, bank from the road towards the airfield, and begin flying a large circle over the airfield. Once the target ground speed was achieved (though the launch vehicle would exceed this by $5 \mathrm{mph}$ for a launch speed of $25 \mathrm{mph}$ for safety), the aircraft would attempt to takeoff despite still being tethered to the launcher. This allows for the passenger to ensure the aircraft is behaving as expected before releasing it, including hearing the motors arm and watching the wings try to leave the launcher. During the launch sequence, the pilot and launch vehicle behaved as normal, with the honking signaling for the pilot to be watching the aircraft in case he needed to take the aircraft out of auto-mode and into manual.

The launch and ensuing flight were very successful and showed this launching procedure had great promise for TIA. Due to the ground speed requirement set in the autopilot, a new standard launch speed of $25 \mathrm{mph}$ was established. There was a slight cross wind $\left(\sim 18 \mathrm{mph}\right.$ at $\left.30^{\circ}\right)$ which caused the aircraft to drift off the road after launching but before reaching altitude. Though the autopilot was likely capable of correcting before being in danger of hitting a utility pole or its wires, the pilot switched from Auto to Manual mode at $50 \mathrm{ft}$ AGL to ensure no damage would be 
caused to the aircraft or the structures. This experience taught that, when in a cross-wind launch scenario, launch where there are either A) no utility poles or wires on either side of the road or B) launch with the structures on the upwind side of the vehicle, in order of preference. If the primary wind direction is from the front left side of the vehicle's launch direction, ensure no structures are on the right side of the road to account for any drift that may occur to the aircraft due to this wind.
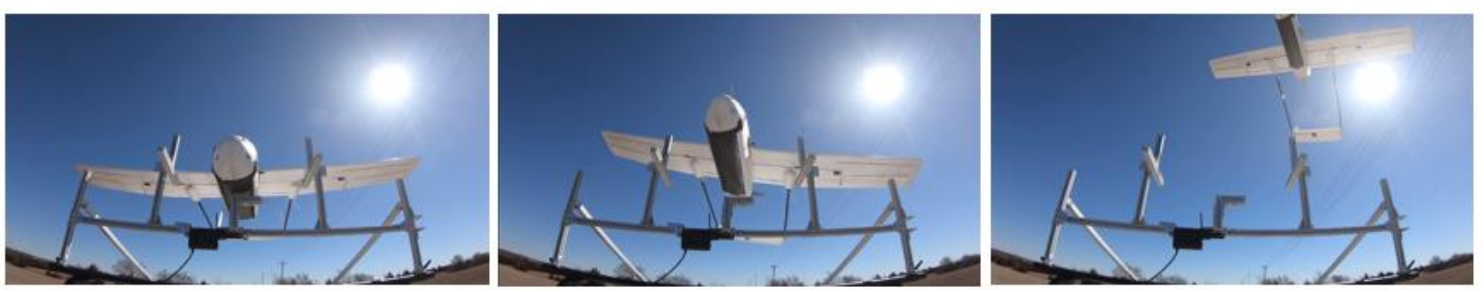

Figure 50: Skyhunter launching in Auto mode

Follow-up tests of the autopilot test was conducted in a low wind scenario $(<5 \mathrm{mph})$, and those tests showed the autopilot is capable of keeping the aircraft over the road for most of the climb out. For safety, all auto-launches will be conducted with at least one side of the road open to allow for the pilot a relief in the event the aircraft drifts in an unfavorable direction.

As a whole, FT2 showed that by allowing the Pixhawk autopilot to control all functions both pre- and post-launch, no pilot input is required until the aircraft is ready to land, as the normal function of the autopilot is for control while already in the air. This drastically reduces pilot input from $100 \%$ of the mission time down to a mere $5 \%$ for a 30 minute mission (assuming 1 minute for landing; lower percent for longer mission).

\subsubsection{5: Flight Test 3: Believer, Dual Prop, Conventional Airframe}

With the single prop, conventional airframe type aircraft well tested and showing very positive results, the next step was increasing from single to dual prop. This keeps the aircraft flight 
dynamics very similar to the previous tests overall while increasing weight yet again and increasing launcher complexity. With pusher prop aircraft, the prop is not in danger of striking any of the launcher's structure. However, with dual tractor (puller) props, the prop disk is moved from behind the wing to in front of the leading edge. This introduces increased risk of a prop strike on the wing support structure of the launcher.

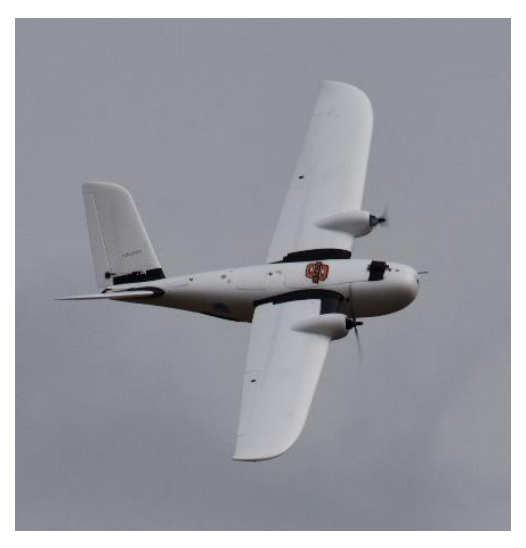

Figure 51: Believer, dual prop UAS

To circumvent this, the launcher's main crossbar was extended, pushing the vertical supports, which hold the wing supports, further outboard on the wing. By pushing them to the furthest allowed on the launch vehicle, an 8-inch gap was created between the outer edge of the prop disk and the nearest vertical support. This was deemed appropriate, as the other aircraft have typically gained altitude before displaying noticeable drift, and the gap reduces the prop strike risk to only in a very strong pure cross wind condition. This condition cannot be achieved, as the vehicle's forward motion prior to release would ensure there will always be some backwards and upward movement of the aircraft on launch.

To promote modularity of the launcher to work with any of the aircraft tested previously and others in the future, the vertical structures supporting the wing platforms were removed from the vertical members anchoring the frame to the roof rails. This new independent vertical member 
is capable of sliding along the length of the extended crossbar, allowing for the previously tested aircraft to launch from this in the future by sliding the supports to be in the same position they were previously. This is advantageous, as now a single system can service multiple aircraft by simply loosening a couple screws and sliding the vertical structures around.

The first set of flights using the Believer (FT3) were conducted in FBWA mode, as FT2 had shown the autopilot assisted modes greatly reduced the effort required by the pilot, reducing input to directional control rather than accounting for every variable load the aircraft experiences. The mission profile was the same as for the Skyhunter, as the two aircraft are very similar with two main exceptions: the Skyhunter uses an H-tail on a boom while the Believer uses a V-tail attached to the fuselage, and the Skyhunter uses a single pusher prop while the Believer uses dual tractor props. The tail difference was not a concern as the likelihood of a tail strike is very low, but the testing and confirmation of a dual prop aircraft would increase the overall capabilities of the launcher.

Like the Skyhunter, the Believer was brought up to $25 \mathrm{mph}$ before releasing and followed the standard launch procedure. As the other FT's before it, FT3 was also a success, as the dual prop displayed no issue with launching and climbing out, and there was no noticeable shift of the aircraft on launch that would put the aircraft's prop disk at risk of colliding with a vertical support. FT3 was noted to be the smoothest launching sequence tested yet, as confirmed by the pilot, passenger, and extra personnel standing near the pilot. This setup was very repeatable and displayed no cause for concern over the multiple (5+) flights conducted in this series. 

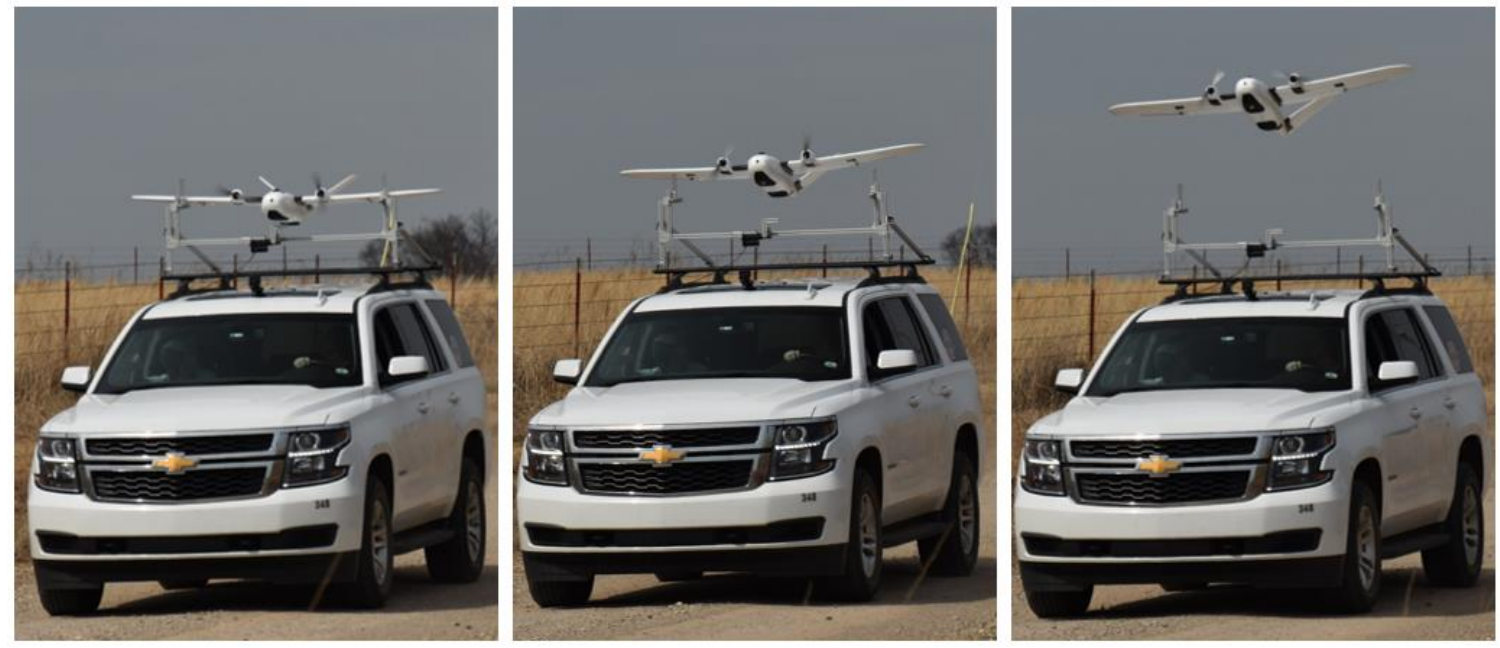

Figure 52: Believer launching, full Auto mode

The next progression from a FBWA launch is another full auto launch, this time with the Believer. The mission programmed into the Pixhawk was very much the same as for the Skyhunter: activate props at 20 mph GPS groundspeed, passenger verify all systems are functioning as expected, driver honks when at $25 \mathrm{mph}$ and near pilot, passenger releases aircraft, and pilot watches aircraft as it climbs out at a $12^{\circ}$ angle of attack to $150 \mathrm{ft}$ before banking toward the airfield for a circling pattern.

The auto launches followed this exact outline, with the aircraft and launcher performing flawlessly. This was also a highly repeatable event and required no pilot input until landing, much the same as FT2 demonstrated but on a new airframe. This final set of flight testing proved the launcher was capable of launching not just single prop, but also dual prop aircraft. There are goals of making MARIA, the 35-pound dual prop UAS ${ }^{11}$ outlined in Section 2.2, with similar prop spacing as the Believer also launch from this system, but that will be a focus of future work.

\subsubsection{6: Flight Test 4: Skywalker X8, Single Prop, Delta Wing}


Following the success of the various conventional style aircraft, the next step was to try airframes similar to TIA. Using a similar step-down method, the test would consist of a foam delta wing aircraft to ensure there is minimal (if any) adverse effects due to wing sweep and having a lower aspect ratio wing planform. The foam aircraft used for this testing was the Skywalker X8 with a $2120 \mathrm{~mm}$ wingspan. The primary similarities were the delta wing planform shape and reliance on winglets for directional stability. The X8 is significantly lighter than TIA (7 pounds vs 20), but the expectation was for it to perform as the Skyhunter and Believer did, as all three aircraft have been used extensively and shown to be reliable aircraft.

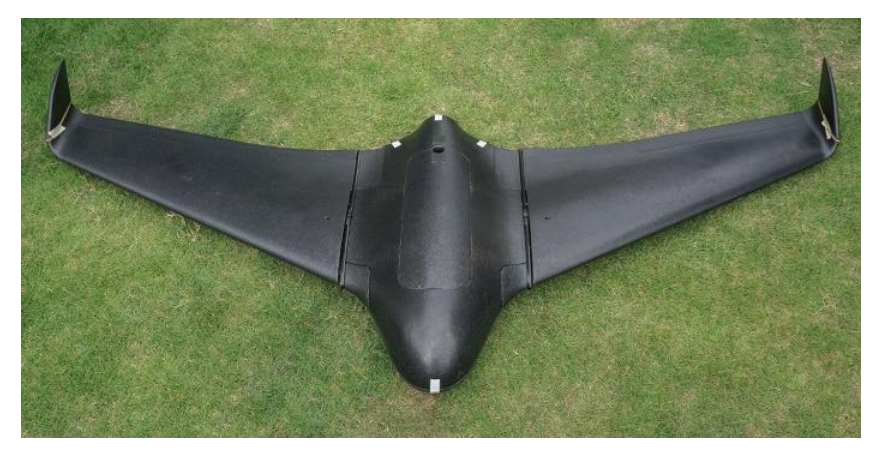

Figure 53: Skywalker X8, 2120 mm wingspan, pusher prop ${ }^{45}$

However, integrating the $\mathrm{X} 8$ with launcher presented a new challenge: preventing the aircraft from yawing while on the launcher. For all the previously tested aircraft, the wings had no sweep, meaning all the leading edges were perpendicular to the flow. This meant that as the launch vehicle begins moving and the aircraft started taking aerodynamic loads, a slight side force would simply slide the aircraft slightly side to side, there would be no rotational moment. However, due to the delta wing's leading edge sweep, as the same side force is imparted on the aircraft, it would pivot around the tether point. The two vertical structures that previously sufficiently held the aircraft became a member for the wing to slide and pivot across. This had a strong potential for one wing to nearly fall off the wing support, which would predictably end in a failed takeoff with a potential for massive destruction to the aircraft. 


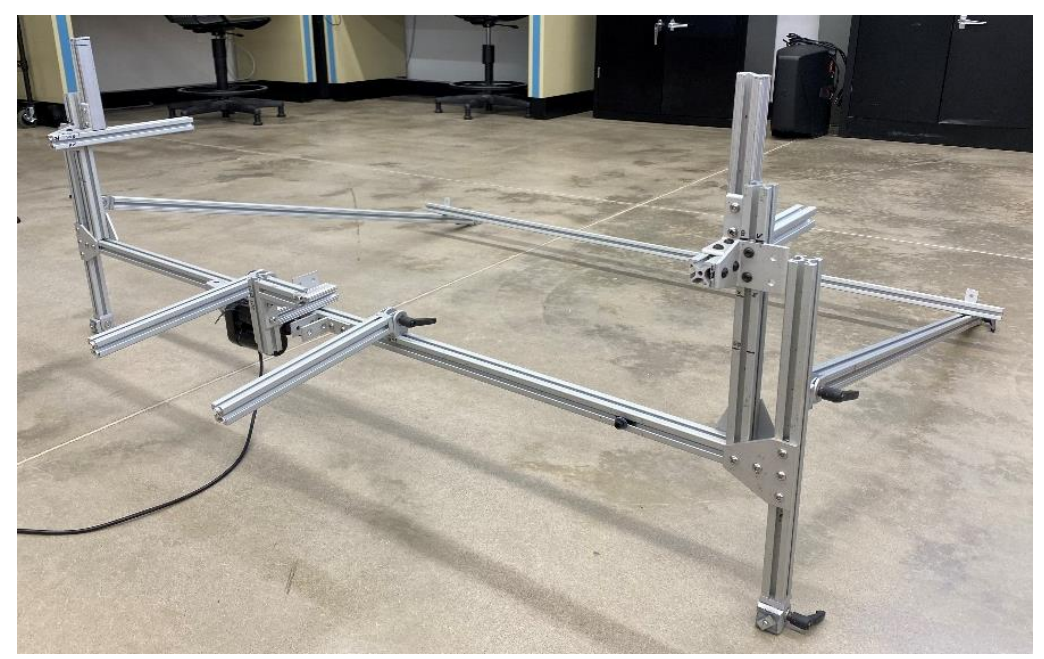

Figure 54: Launcher with delta wing modification installed

To supplement this, additional structure was mounted on either side of the anchor point with the structural lengths holding the aircraft's nose forward of the anchor point. These bars were attached to pivots to allow for a fine-tuned fit prior to each launch, meaning it also would allow for a multitude of wing sweep angles. The pivots were added so the aluminum bars could be locked in a horizontal orientation to remain clear of all components for conventional propeller aircraft launches or in varying degrees of vertical for any degree of sweep for delta/swept wing configurations. This is important, as TIA and the X8 did not have the same sweep angle, and the launching system must be modular to work with any aircraft with minimal adjustments between launches. The structure was placed in a position on either side of the pivot point such that it would pinch the nose of the aircraft. Should a side load act on the aircraft, as the wing attempts to slide, the nose portion contacts the support, preventing the aircraft from rotating on the launcher. This helps the aircraft remain directionally stable the entire time it is secured to the launch vehicle. With this modification in place, the $\mathrm{X} 8$ was placed on the launcher and the supports tuned to hold the aircraft stable. 

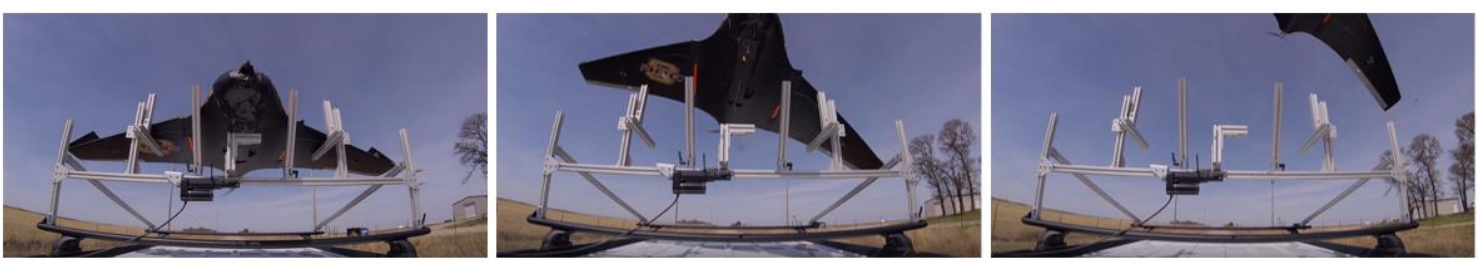

Figure 55: X8 launch sequence

The flight plan for the $\mathrm{X} 8$ was to launch in full manual (no autopilot or rate gyro installed) to see the full aerodynamic effects of the delta wing planform on launch. As with the previous tests, the aircraft would be secured as before, with the addition of ensuring the front bracers were set properly to ensure the aircraft did not yaw while on the launcher. The launch vehicle would begin driving, a honk would signal to the pilot release was imminent, followed by releasing the aircraft. The pilot would then climb out until a proper altitude is reached (150-200 ft, same as the future auto-missions would be set for), then bank towards the airfield and set up for landing.

The aircraft was then put through this series of flight testing following this guideline. Overall, the aircraft performed very well, though much was learned. In each test, the aircraft seemed to roll to the left (port) after the release, which while perfectly fine for the $\mathrm{X} 8$ with a wingspan wider than the launcher's base, could be a problem for other similar aircraft whose wingspan is less than the launcher frame's stance, such as TIA. There are two potential reasons for this roll effect. The first is roll induced from the propeller's rotational direction. From observing the propeller while active, the prop spins in the opposite direction as the roll exhibited in the videos, which supports this ideology. This implies the torque generated by the motor to maintain motion of the propeller is causing this roll on release. The second reasoning could be a partial stall on the port wing/partial increase in lift on the starboard wing caused by off-centered wind gusts. Though the winds were observed to be approaching roughly $30^{\circ}$ from centerline of the aircraft on the front starboard side at $\sim 10 \mathrm{mph}$, various wind speeds and directions were observed, and the same effect held true through all tests except for the Believer. This implies that though the winds could 
be causing a partial stall, it is more likely that the ambient winds amplify the effect of the torqueinduced roll. There are other potential factors, such as gyroscopic precession and p-factor, but those are deemed minimal due to the low rotational speeds of the motors and the presence of the roll across both props vehicles, respectively.
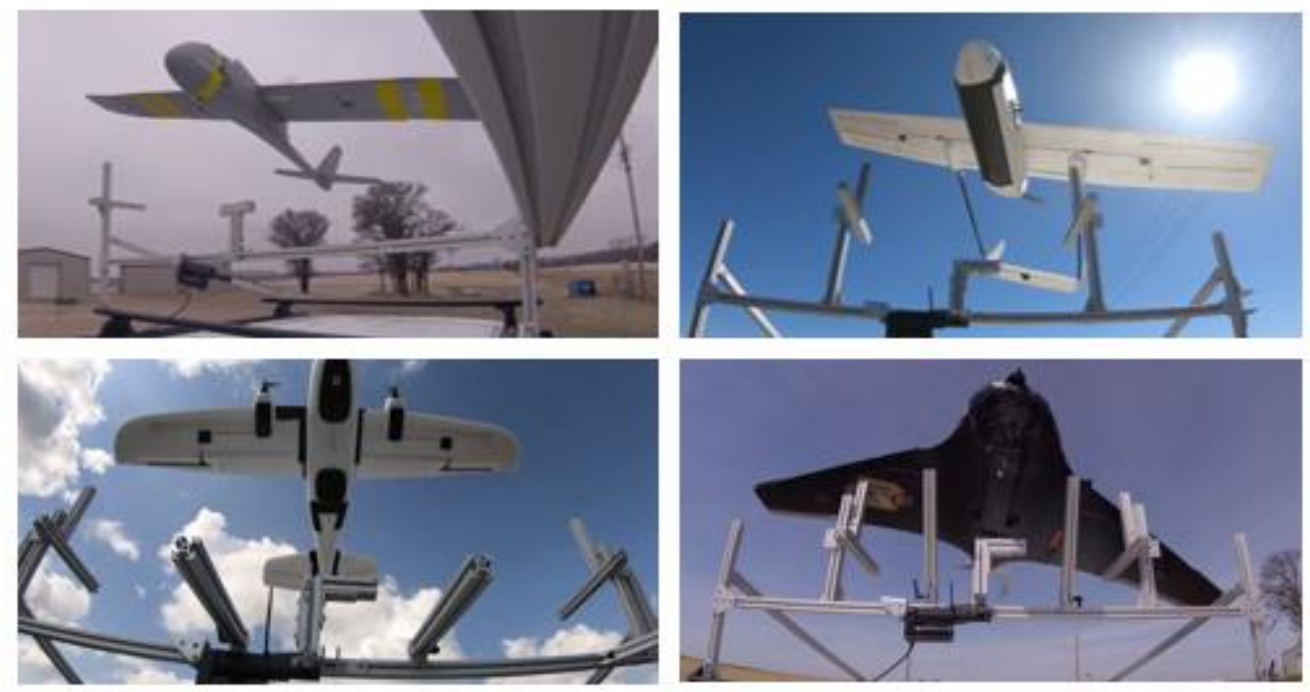

Figure 56: All four tested aircraft exhibiting roll on release except for the Believer (lower left)

Of the rationales provided above, both are valid to differing extents. The first reasoning is likely the primary culprit, as each of the aircraft with a single propeller experienced a more severe rolling motion than the dual prop aircraft (Believer). A single prop acting along the center line would produce a pure rolling moment, whereas a dual prop aircraft spins each propeller in opposite directions, which minimizes this roll effect experienced by the airframe. This would describe why only the Believer did not experience a noticeable roll on takeoff. The second reasoning was present in some of the other aircraft tested (cross-wind of varying degrees in all tests), but as the rolling effect maintained a fairly constant angle and severity despite the differing cross wind conditions and wing types, this seems to be a propulsive effect influencing the immediate post-launch aerodynamics rather than a partial stall caused by ambient wind. 
Due to the successful set of X8 flights, delta wings were determined to be capable of successfully launching from the launcher. Unlike the conventional airframes tested prior, the X8 was not tested with autopilot integration. The conventional airframes included in the previous testing are among some that will be routinely launched from the launcher in addition to TIA for storm observations. The X8 will not be on that list as it does not perform as well as the other aircraft, and for this reason it was not pursued further. Instead, the next step was to launch the TIA electric ducted fan (EDF) prototype: Beast. Instead of a diesel-burning turbojet as TIA utilizes, Beast features an all-electric propulsion system that relies on two 4S $5500 \mathrm{mAh}$ batteries wired in series for power, which effectively becomes a single $8 \mathrm{~S} 5500 \mathrm{mAh}$ battery. The EDF, which is a modified airframe variant of the turbojet version, allows for similar aerodynamic analysis while drastically reducing replacement cost should something go wrong, as the EDF costs $\$ 200$ compared to the $\$ 2000$ price tag of the turbojet system.

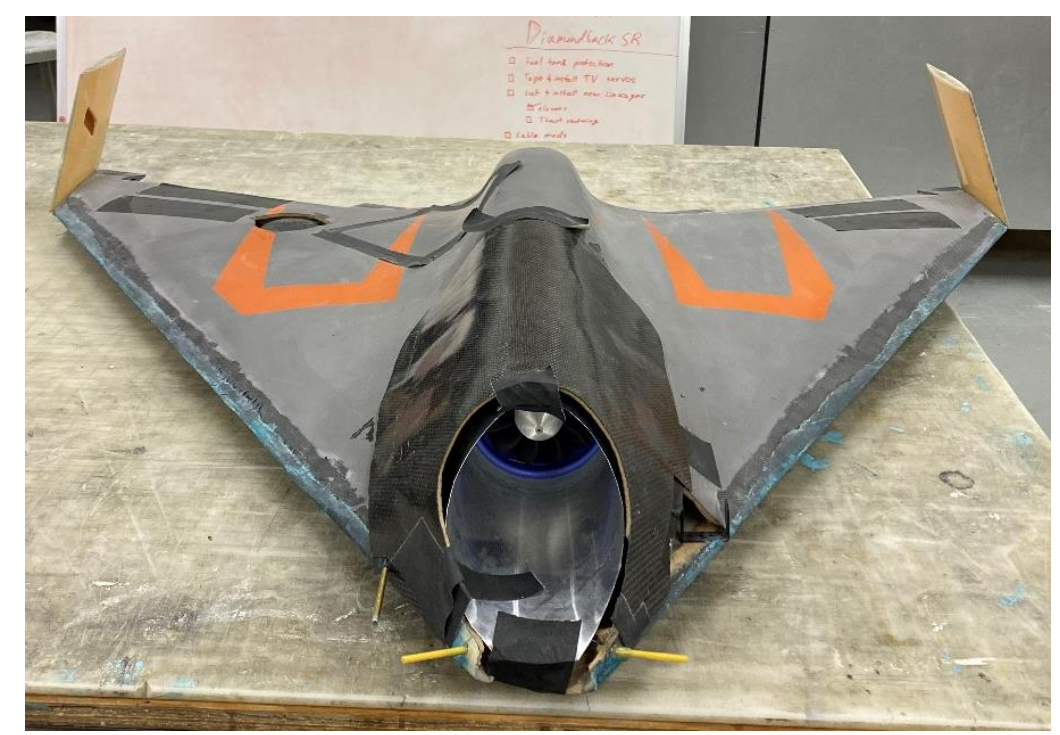

Figure 57: Beast, EDF prototype of TIA 
The overall planform and structure of Beast is identical to TIA, as they were both created from the same mold. Structurally, they differ slightly. TIA features a 2-core-1 layup of $3 \mathrm{oz} / \mathrm{yd}^{2}$ fiberglass whereas Beast uses a 1-core-1 layup of $2 \mathrm{oz} / \mathrm{yd}^{2}$ carbon fiber. The core material remains constant and is a $1 / 8$ " Divinicell closed cell foam. Internally, the only major difference is propulsive types. Whereas the turbojet features a centrifugal compressor, meaning it does not require axial flow but rather a total inlet area to draw in air from, the EDF does require clean, axial flow through the fan blades. Having a "clean" airflow means the streamlines are straight from the external flow field all the way to the front face of the EDF's fan disk.

This required the removal of much of the nose of the aircraft to promote clean airflow to the EDF. To further prevent the airflow from tripping into turbulent over the various components that were still housed in the nose, an aluminum inlet was placed in the nose from the airframe's new edges to the EDF's face. This kept the surfaces as smooth as possible, promoting the clean airflow that is sought after.

Other than the propulsive differences and weight (TIA: $20 \mathrm{lbs}$, Beast: $11 \mathrm{lbs}$ ), TIA and Beast are very similar aircraft. In its initial flight tests, the autopilot and FPV video systems were removed but the Aura 8 rate gyro remained installed. The autopilot and FPV were removed to reduce cost in the event a crash ensued, as the autopilot system cost $\$ 350$ and the FPV system $\$ 200$. The Aura 8 rate gyro remained installed as it was relatively cheap $(\$ 100)$ and ensured the pilot would have as high of a chance as possible to recover the aircraft should the launch be imperfect. The original systems TIA is derived from all utilized the Aura 8 on its own, so there was a lot of reliability stemming from the Aura 8 rate gyro being installed in this particular airframe. 


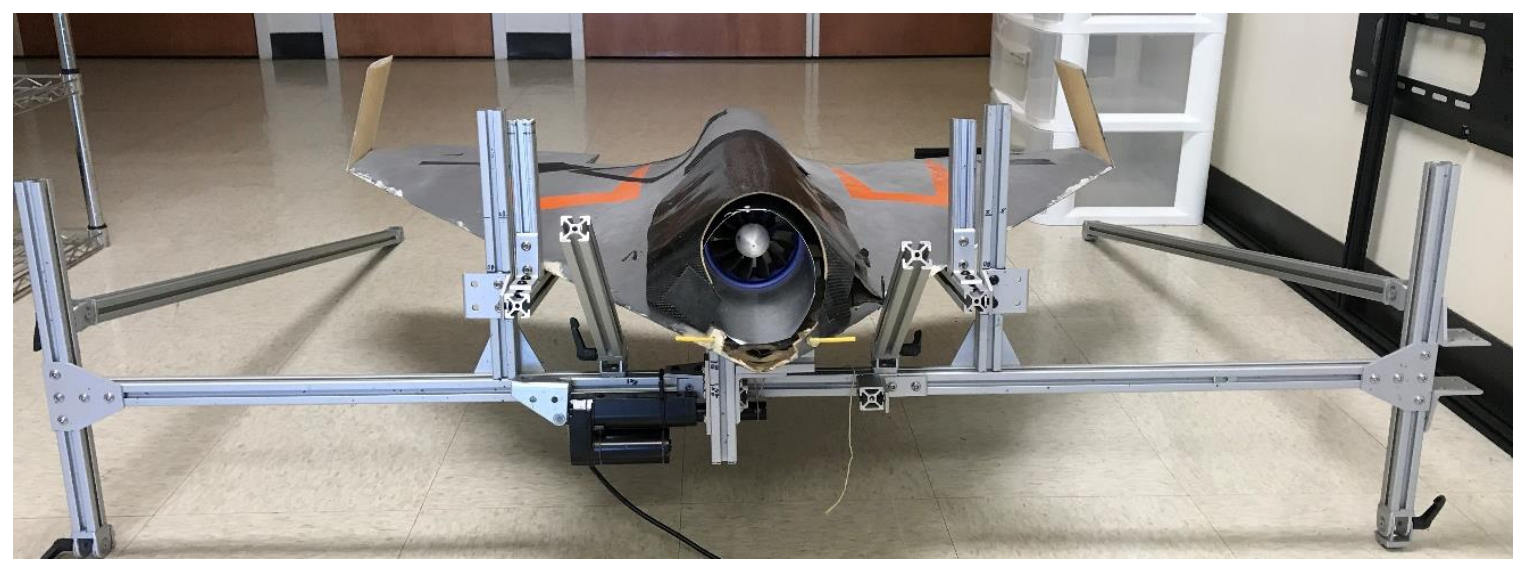

Figure 58: Beast on launcher for fine-tuned placement of all support structure

As previously mentioned, the first set of flight tests with Beast were done in full manual mode. The flight profile was the same as for all the other aircraft, but due to the lower aspect ratio of the wing and heavier overall weight compared to the X8, the launch speed was increased from $25 \mathrm{mph}$ to $35 \mathrm{mph}$. The lower aspect ratio inherently requires a higher launch velocity than a higher aspect ratio wing, and that coupled with a heavier aircraft, which requires more lift, were the two defenses for increasing the launch speed. Beast was attached to the launcher and followed the same flight procedure and profile as mentioned for all the other flight tests.

The very first launch attempt did not go as anticipated. The launch vehicle was brought up to speed, the aircraft was set to max throttle, and the aircraft released, but it did not take off. Rather, the aircraft remained pinned against the vertical supports with no attempt to actually take off. As the launch vehicle was brought to a stop, the aircraft was inspected and there were no physical snags preventing the aircraft from taking off. After review with the pilot, it was deemed to be a pilot error, as the pilot was not inputting enough pitch deflection to the servos. This was an important note, as this aircraft in its base state requires more elevator deflection than any of the foam aircraft did. 
With this in mind, the aircraft was prepped and reloaded. For this next flight, the pilot would keep in mind the extra elevator required and determine was needed. The aircraft was then relaunched. This time the aircraft did take off, but very slowly. The pilot began with minimal elevator input, but quickly increased in pitch to $50 \%$ of the max control input, which allowed the aircraft to take flight. The takeoff wasn't smooth, but it was learned that for that launch speed, a significant elevator deflection is needed (50\% minimum compared to the $20 \%$ for the foam aircraft). The aircraft was able to climb out, loiter, then land with no issue.

Though the flight was a success, it exhibited the same roll movement in the same direction as what was noted in the X8 takeoff. Though the X8 had a wide enough wingspan to prevent any interaction with the structure, the Beast/TIA airframe does not share that quality. The Port winglet on Beast came close to sliding under the bracer that holds the launcher's front legs vertical. Had this happened, the aircraft would have gotten stuck there, either pivoting at the winglet and crashing right beside the vehicle and being at risk of being run over, or being fully caught and restrained on the top of the launch vehicle before sliding off in an unknown direction. This is a severe threat to the aircraft as a whole, and the initial reaction was to pause flight testing until the underlying issues were addressed. 

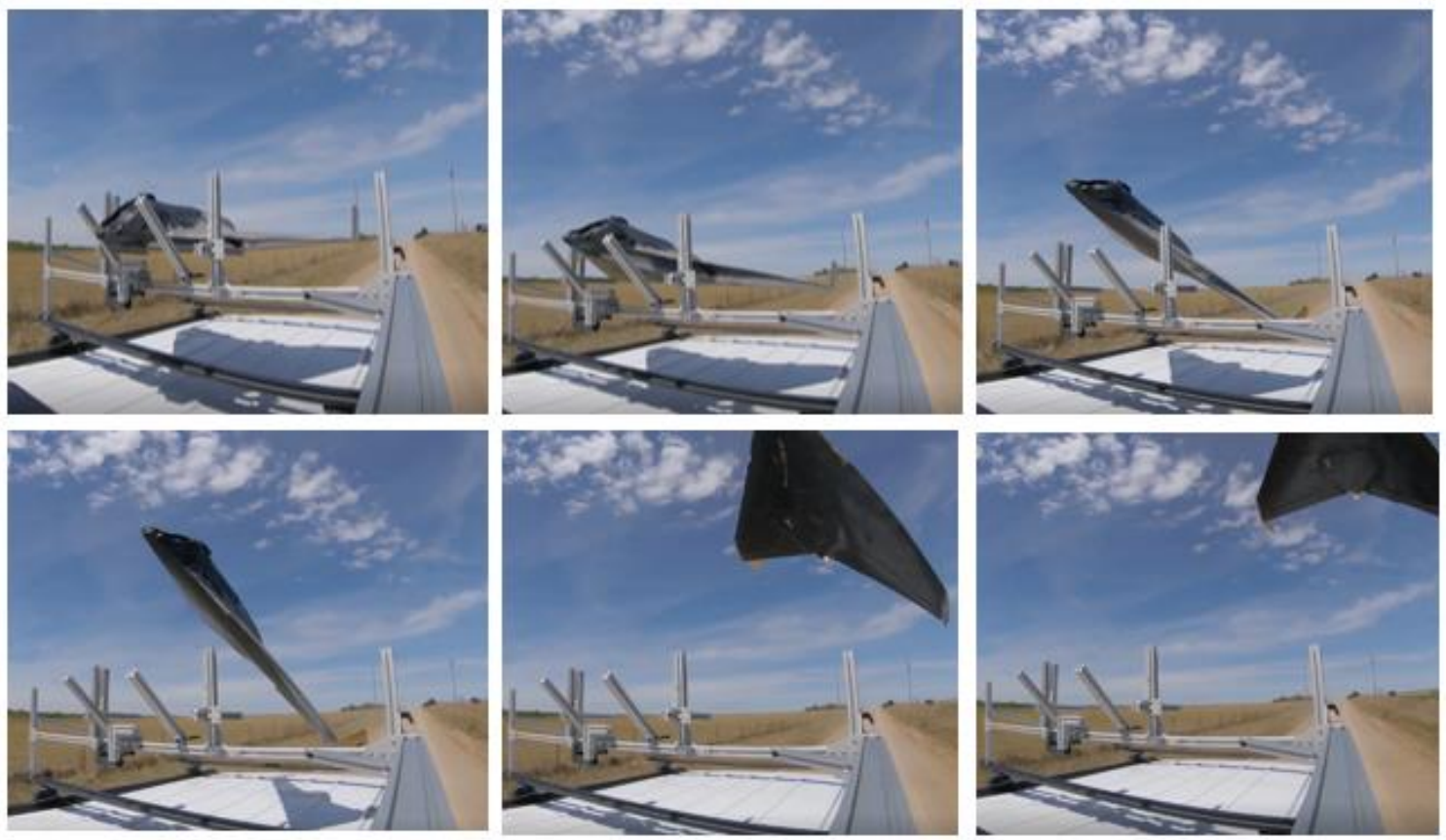

Figure 59: Beast launch, severe roll on takeoff with rapid recovery

Also present was another cross-wind gust, this time at approximately $7 \mathrm{mph}$ at a $45^{\circ}$ heading from the centerline on the front starboard side. Though this off-axis gust was present, lower intensity gusts were also tested with the same severity in roll on release, furthering the idea that the roll seen in the release of previous aircraft was due to the torque supplied from the EDF's motor. Since this effect was also seen in varying degrees on all other tests (none on Believer, minimal Firstar and Skyhunter, noticeable on X8) coupled with the severity of the effect on Beast, an estimated comparison between the different aircraft was created to attempt to quantify the trends influencing this issue. Of note: since the full version of TIA uses a turbojet, there will not be a torque effect seen on launch with it, but as other electric prop aircraft will routinely be launched as well, this estimation is required. 


\begin{tabular}{|c|c|c|c|c|c|c|c|c|c|c|c|}
\hline Aircraft & $\begin{array}{c}\text { RPM } \\
\text { (per motor) }\end{array}$ & $\begin{array}{c}\text { Max } \\
\text { Power (W) }\end{array}$ & $\begin{array}{l}\text { Torque } \\
\text { (ft*lbf) }\end{array}$ & $\begin{array}{c}\text { Wingspan } \\
(\mathrm{ft})\end{array}$ & $\begin{array}{c}\text { Weight } \\
\text { (lbf) }\end{array}$ & $\begin{array}{c}\text { CG Depth } \\
\text { (ft) }\end{array}$ & $\begin{array}{l}\text { Iy Fuselage } \\
\left(\text { (lbf* } \mathrm{ft}^{2}\right)\end{array}$ & $\begin{array}{c}\text { Wing } \\
\text { Weight } \\
\text { (lbf) }\end{array}$ & $\begin{array}{l}\text { ly wing } \\
\left(\mathrm{lbf}^{*} \mathrm{ft}^{2}\right)\end{array}$ & $\begin{array}{l}\text { Iy Total } \\
\left(\mathrm{lbf}^{*} \mathrm{ft}^{2}\right)\end{array}$ & $\begin{array}{c}\text { Torque / Iy } \\
\text { (1/ft) }\end{array}$ \\
\hline Firstar V2 & 13200 & 500 & 0.27 & 6.56 & 3 & 0.5 & 0.75 & 1 & 3.59 & 4.34 & 0.06 \\
\hline Skyhunter & 12400 & 870 & 0.49 & 5.9 & 8 & 0.5 & 2 & 1 & 2.90 & 4.90 & 0.10 \\
\hline Believer & 15500 & 770 & 0.35 & 6.43 & 12 & 0.5 & 3 & 1 & 3.45 & 6.45 & 0.05 \\
\hline Skywalker X8 & 12400 & 870 & 0.49 & 7 & 6 & 0 & 0 & 1 & 4.08 & 4.08 & 0.12 \\
\hline Beast & 28200 & 2880 & 0.72 & 3.7 & 11 & 0 & 0 & 2 & 2.24 & 2.24 & 0.32 \\
\hline
\end{tabular}

Table 6: Estimated aircraft torque and inertia values

The torque values were gathered by approximating the RPMs each aircraft's motors spin up to on takeoff (max throttle). Using the KV rating multiplied by the volts supplied by the batteries, the RPM can be found. The Max Power, which is listed for a given motor, was then divided by the max RPM, which when converted properly, yields the estimated Torque provided on takeoff.

The moment of inertia was estimated in two steps: first the fuselage portion, then the wing contribution. The fuselage portion was estimated by using the radius of the fuselage and the total weight of the aircraft and using the following equation:

$$
I_{\text {fuselage }}=\frac{m r^{2}}{2}
$$

Where $\mathrm{m}$ is the aircraft's mass (weight in this case) and $\mathrm{r}$ is the radius of the fuselage. For the approximation, the aircraft's total weight was used, which does introduce error, but will still allow for the trends to be revealed. For the wings, two steps were used. The first was the moment of inertia of the wings themselves, which for a flat plate out its longitudinal axis around its center of mass is:

$$
I_{\text {wing } C o M}=\frac{m b^{2}}{12}
$$

Where $\mathrm{m}$ is the estimated mass of the wings themselves and $\mathrm{b}$ is the wingspan. Additional error was introduced in estimating the weight of the wings, but all the wings are made of the same EPO foam and have similar total wing areas (except Beast). This allowed for the foam aircraft to have 
the same margin of error, meaning they could be compared despite having error from the true value. The final component was the moment of inertia from the wings being offset of the centerline of the aircraft.

$$
I_{\text {parallel axis }}=m r^{2}
$$

Where $\mathrm{m}$ is the mass of the wing and $\mathrm{r}$ is the radius of the fuselage. All three of these individual components were added together to be the total estimated lateral moment of inertia. There is much error, approximated to be as high as $25 \%$ due to the estimated weight metrics, but even with this large maximum error bound, trends still arise: the larger the wingspan and aspect ratio, which results in a larger lateral moment of inertia, the lower the Torque/Lateral Moment of Inertia value is.

Though the Torque/Lateral Moment of Inertia is the likely parameter the governs the effect seen and an approximation was shown above, these data points are very inaccurate. To supplement this, a frame-by-frame video analysis was conducted to analyze the roll angle and roll rate. The roll angle was tracked by using two points in a straight line on the aircraft, and as the frames changed, the pixels occupied by the points changed. This change in $\mathrm{x}$ and y pixel coordinates were then converted into angles. Since the camera was shot at 60 frames per second, each frame represents $1 / 60$ seconds of time. Using these pieces, the below data was gathered.

\begin{tabular}{|c|c|c|c|}
\hline Aircraft & Total Roll & Roll Time & Roll Rate \\
\hline Skyhunter & $7^{\circ}$ & $0.13 \mathrm{sec}$ & $50^{\circ} / \mathrm{sec}$ \\
\hline X8 & $26^{\circ}$ & $0.25 \mathrm{sec}$ & $103^{\circ} / \mathrm{sec}$ \\
\hline Beast & $43^{\circ}$ & $0.25 \mathrm{sec}$ & $172^{\circ} / \mathrm{sec}$ \\
\hline Believer & $1.5^{\circ}$ & $0.167 \mathrm{sec}$ & $9^{\circ} / \mathrm{sec}$ \\
\hline Firstar V2 - slack in cable & $3.4^{\circ}$ & $0.083 \mathrm{sec}$ & $41^{\circ} / \mathrm{sec}$ \\
\hline Firstar V2 - on release & $.1^{\circ}$ & $0.067 \mathrm{sec}$ & $1.6^{\circ} / \mathrm{sec}$ \\
\hline
\end{tabular}

Figure 60: Roll angles and rates from frame-by-frame video review of launched aircraft 
The Firstar V2 has two data entries due to the poor performance of the initial version of the launcher. There was sufficient slack for the aircraft to attempt a takeoff while still tethered. As such, the roll the aircraft attempted during this step was recorded as "Firstar V2 - slack in cable" and the actual roll on release from the launcher was recorded as "Firstar V2 - on release". The rest of the aircraft have only one data entry since they launched without issue, as the problems with the initial version had since been resolved.

The implications as applied to the aircraft on launch is that while the cross wind may be amplifying the roll out effect, the majority of it is started due to the torque imparted from the spinning of the propulsion system. The EDF has the most severe Torque/Lateral Moment of Inertia metric, and it also has the highest rotational rate of all tested aircraft, spinning at rate of up to 28,000 RPMs. At the moment the aircraft is free of the tether and wing supports, there is nothing resisting the fan blades' rotational inertia, causing the airframe to roll in the opposite direction of the propulsion system (propeller, EDF, etc.). The theory aligns well with the test data: for all single propulsive systems, there is a roll on release that is not present in any of the dual prop flight data despite operating in very similar ambient environments. This implies that any planform with a single propulsive system (conventional or delta wing) that imparts substantial torque on a propeller or fan will experience some degree of roll on release, with the magnitude of the effect directly related to the total torque and effective wind speed and direction the aircraft is released in, whereas a dual prop system will be minimally influenced by the torque effect, as the two propellers rotating in opposite directions severely reduces (though does not fully remove) this effect. These implications can be directly observed from the various test points gathered from the launches. This occurrence is not anticipated to be an issue for the turbojet powered TIA as the jet's propulsive system is not powered via torque, but rather from the airflow generated by the jet's operation. 


\subsection{TIA Flight Testing}

Though TIA was not launched from the launcher due to time constraints, the aircraft did undergo flight testing of its own. Returning to its origins, the aircraft was hand-launched with a high angle of attack landing. The Pixhawk on board gave valuable insight that was used for in the Section 4.5, Controls Analysis.

\subsubsection{TIA Maiden Flight 1}

The first maiden flight was intended to be a full systems check, including ensuring all PID gains were set appropriately and that the aircraft as a whole tuned properly. Should anything visibly look wrong of the pilot feel uncomfortable with an aspect of flight, the aircraft would quickly be brought down for inspection, as is typical of all maiden flights. To do this, the aircraft was to be hand launched, climb to altitude in full manual mode, then switch to FBWA for a couple passes, then switch to Auto mode (auto mission was the same loop) before going back into FBWA for landing.
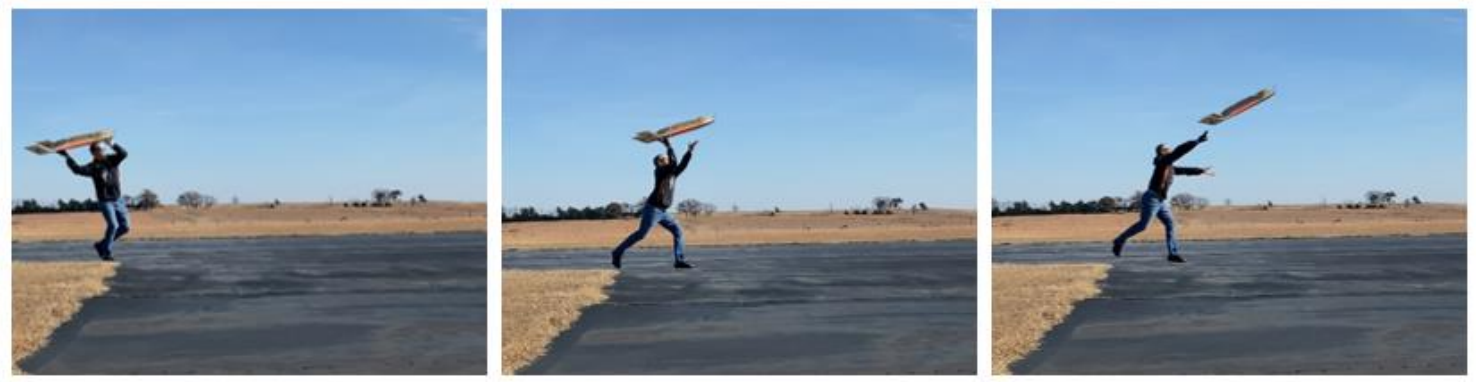

Figure 61: Hand launch for TIA's maiden flight 
The overall plan started as expected. The aircraft launched perfectly, climbed out, and managed to pass through all the mode switches. However, as the pilot transitioned to FBWA while still loitering, a small piece of epoxy that remained in the fuel tanks dislodged and entered the fuel pump, killing it immediately. This caused the turbine to fail, which was noted by the large reduction in noise, as the turbine sounds like a commercial turbine when in the air.

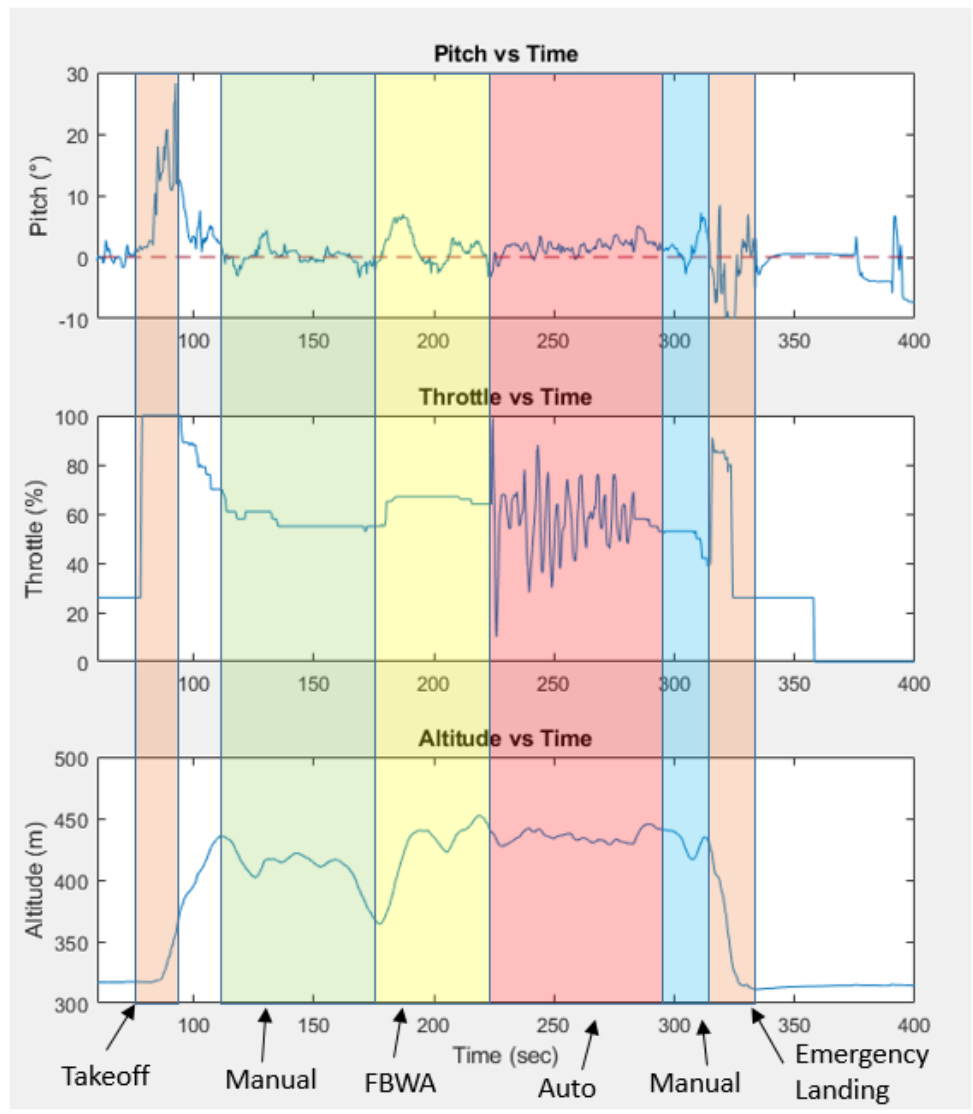

Figure 62: Maiden 1 flight sequence of TIA

Due to the pilot's abilities, the overall handling qualities of the aircraft, and the rigid structure of the airframe, TIA was safely landed with no damage to the airframe despite an emergency landing that involved a turbine out scenario at $500 \mathrm{ft}$ in altitude. To combat any future incidences involving the fuel pump, a second in-line filter was installed on the up-stream side of the pump, allowing for fuel on both the inlet and outlet lines of the pump to be filter. This 
prevents any debris from reaching the turbine or the pump, preserving the integrity of the entire propulsive system. To ensure this did not cause a detrimental loss in fuel flow, ground tests were conducted to check for fuel flow rate, but there was not a significant change. With the aircraft partially flight proven, the re-maiden was then attempted.

\subsubsection{TIA Maiden Flight 2}

Due to the partial success of the first maiden, it was deemed necessary to re-maiden the aircraft before conducting further flights. With the dual fuel filters installed (as can be seen in Figure 40) and ground testing to confirm no ill-effects arising from the dual setup, the aircraft itself was prepper for re-launch. However, to get the most use out of the flight, all water shielding methods were installed. This means the bath tub sealant "gasket" around all the hatches and FPC camera were installed, as were the nose and rear avionics bay Mylar shielding pieces, which can be seen in Figure 41 and Figure 42. With these considerations installed, the jet was ground tested once more to ensure the Mylar would not melt during prolonged operation and that the sealant around the hatches would not choke out the turbine, as there is a slight air intake from around the hatches when there is no sealant. There was no visible damage to the Mylar even after a 5 minute full throttle burn, and the turbine did not show any signs of choking due to the sealant, so the aircraft was deemed ready for Maiden 2.

The aircraft was then prepped to go through the exact same flight sequence as Maiden 1 with the goal of not having any random issues pop up. The flight went exactly as expected: hand launch got the aircraft in the air, flew in circles overhead while cycling through Manual, FBWA, and Auto, then back to FBWA for landing. The landing sequence followed the ideal high angle of attack profile, but in the effort to reduce wear and tear on the system, the aircraft was leveled out before actual landing to ensure no internal components were jarred. A true high angle of attack 
landing is only truly necessary when there are no other safe alternatives, a semi-conventional landing is always preferred with this airframe to reduce impact on the bottom skin.

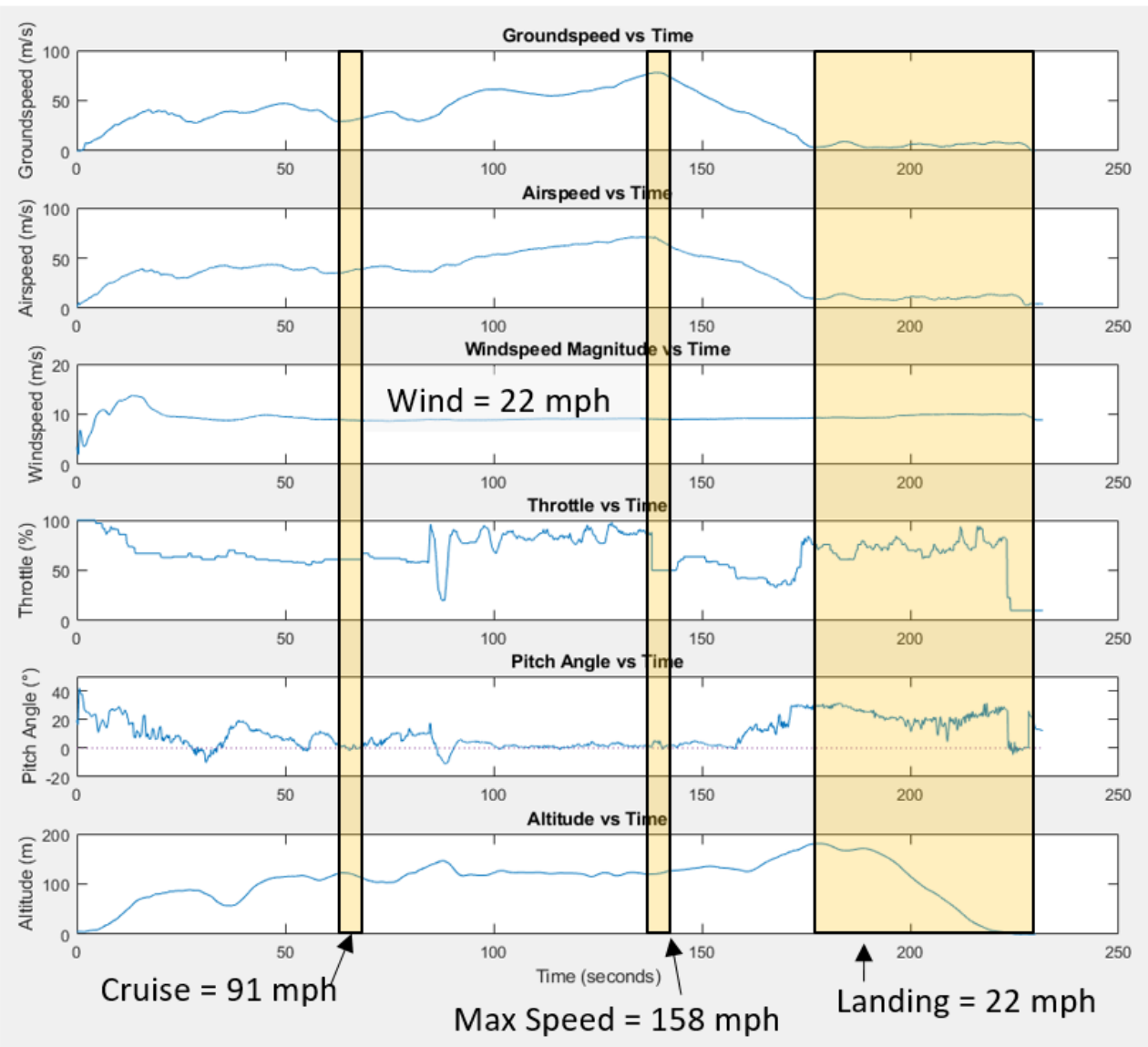

Figure 63: Pixhawk logs from Maiden 2

During Maiden 2, a new top speed metric was achieved, reaching $158 \mathrm{mph}$ airspeed (groundspeed of $175 \mathrm{mph}$ ) at only $90 \%$ throttle. The above diagram is directly from the autopilot's log with each pertinent flight regime noted. These will be used for further analysis in Section 4.5. Of note is the landing airspeed in comparison to the ambient wind speeds. The aircraft was coming down nearly completely vertically, as can be cross-checked with the groundspeed (Figure 63, top graph) and altitude (Figure 63, bottom graph) plots. The steady decrement of altitude coupled with the near-zero ground speed verify no forward or lateral 
movement with a controlled decent until the aircraft lands safely on the ground. The pitch angle plot (Figure 63, lower middle graph) shows this was made possible by maintaining a high angle of attack, keeping an angle between 20 and 30 degrees, but generally increasing until landing. In this case, before touchdown the aircraft was leveled off to prevent unnecessary damage from this maiden flight.

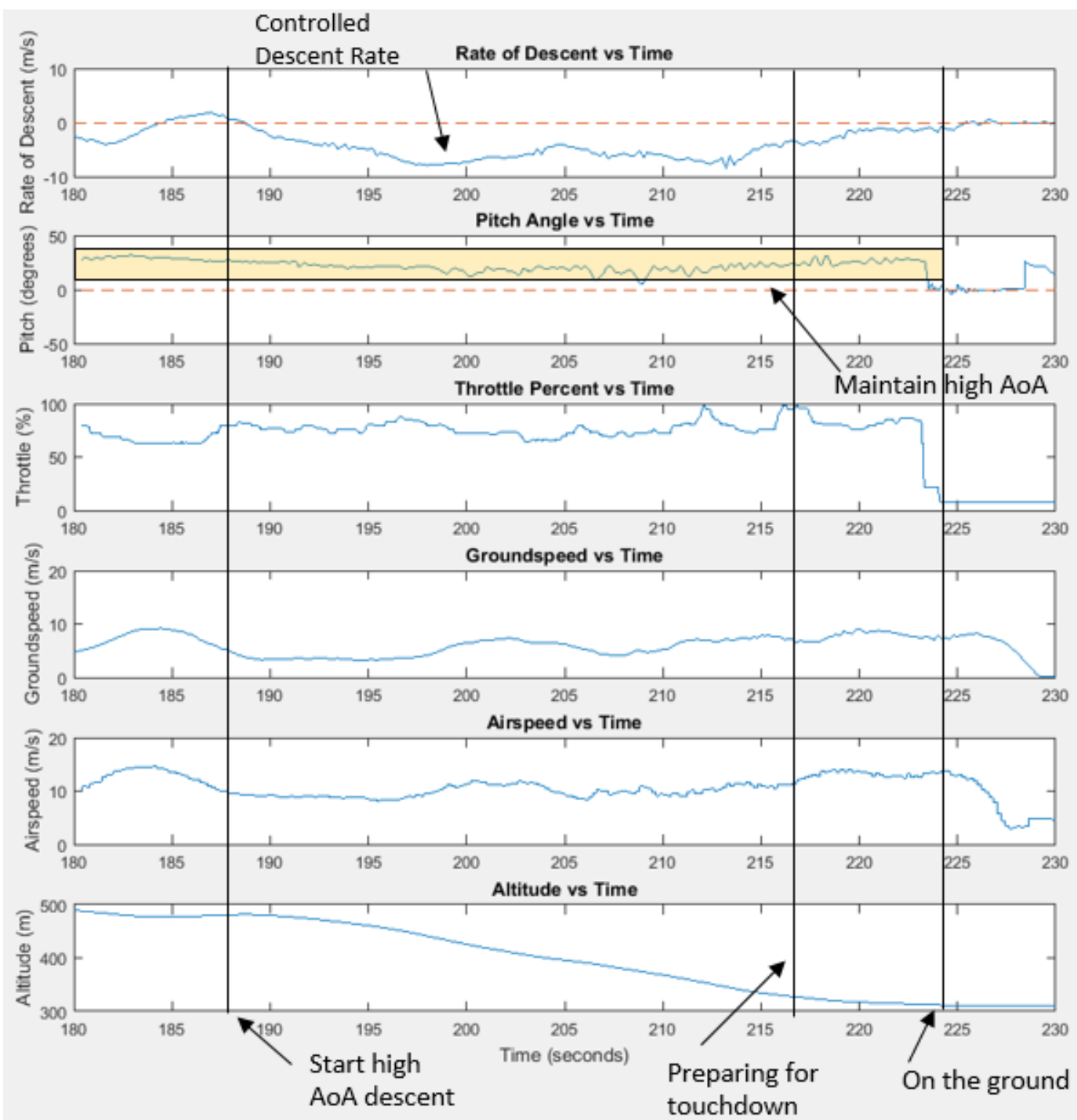

Figure 64: TIA Maiden 2 landing sequence 
Figure 64 is an enlarged plot of Figure 63 with emphasis drawn to the important portions of the landing sequence. From the expanded graph, it is easy to see that TIA was able to be brought down very slowly, bleeding altitude very effectively without increasing airspeed or groundspeed. The smallest total landing area achieved across the entire family of TIA/Diamondback aircraft was under 10 feet, even including the time it took for the aircraft to stop sliding on landing. Due in large part to the thrust vectoring and the presence of the gyros (Pixhawk and Aura 8), the avionics handles all of the flight control, allowing the pilot to simply focus on the rate of descent. Should the landing area be unimproved or hazardous, this technique can be used all the way to the ground to prevent sliding across terrain that could damage the airframe. If the landing area is favorable for a semi-conventional landing, a faster, lower angle of attack landing is attempted to reduce wear of the airframe.

\subsubsection{Maiden 2 Flight Data}

To further analyze the aircraft's performance, data points were captured from these graphs and used for analysis. Of note, all data points were taken from the on-board Pixhawk 2.1 autopilot's data flash logs. A Matlab script was written to parse out the necessary parameter values, light processing to align all data sets, then plotted for visual reference, as seen in the previous section. Those same data points were then extracted for analysis. The primary data in consideration were airspeed, throttle percent, and pitch angle. A constant weight of 16 pounds

was used for all calculations, though this introduces some error (at most $10 \%$ for $C_{L}$ and $C_{L} / C_{D}$ ), the sensor data error is much lower.

Thrust data was gathered from the same turbine used in TIA on a thrust cell using an Omega LCCD-100 S-beam load cell, which has an accuracy of $0.03 \%$ of the reading. 
Assuming quazi-steady state flight profiles, the throttle percentages were mapped to thrust outputs, which were then taken as the instantaneous drag values for that flight profile. The airspeed was gathered via the Pixhawk pitot tube, was has an accuracy of $0.84 \mathrm{~Pa}$, which equates to a max uncertainty of $\pm 3.84 \mathrm{ft} / \mathrm{s}$. These values were used directly. The pitch angle was gathered using the PWM output signal for elevator deflection as recorded by the Pixhawk, which results in a near-perfect result. To equate this PWM signal to an angular deflection, the pitch thrust vectoring servo's deflection was recorded every 50 PWMs from max to min endpoints. The accuracy of these angular deflections within $1 / 32$ ", which equates to a max uncertainty of $0.8^{\circ}$ for the pitch thrust vectoring deflection. The gathered data in tabular form is:

\begin{tabular}{|c|c|c|c|}
\hline $\begin{array}{c}\text { Airspeed } \\
\text { (ft/s) }\end{array}$ & $\mathrm{CL}$ & $\mathrm{CD}$ & $\mathrm{CL} / \mathrm{CD}$ \\
\hline 35.4 & 1.960 & 0.951 & 2.061 \\
\hline 39.9 & 1.363 & 0.678 & 2.010 \\
\hline 98.1 & 0.228 & 0.099 & 2.308 \\
\hline 37.1 & 1.644 & 0.835 & 1.969 \\
\hline 170.7 & 0.074 & 0.033 & 2.285 \\
\hline 231.1 & 0.040 & 0.028 & 1.456 \\
\hline 216.2 & 0.046 & 0.031 & 1.475 \\
\hline 171.8 & 0.073 & 0.053 & 1.386 \\
\hline 137.5 & 0.115 & 0.048 & 2.403 \\
\hline 140.5 & 0.111 & 0.043 & 2.589 \\
\hline 116.4 & 0.161 & 0.065 & 2.465 \\
\hline 200.1 & 0.054 & 0.036 & 1.510 \\
\hline 47.9 & 0.949 & 0.386 & 2.459 \\
\hline 35.5 & 1.871 & 0.721 & 2.594 \\
\hline 231.8 & 0.040 & 0.027 & 1.461 \\
\hline 151.8 & 0.094 & 0.025 & 3.843 \\
\hline 121.0 & 0.149 & 0.058 & 2.595 \\
\hline 178.5 & 0.068 & 0.048 & 1.415 \\
\hline 29.0 & 2.889 & 1.332 & 2.169 \\
\hline 86.8 & 0.291 & 0.063 & 4.593 \\
\hline 118.5 & 0.155 & 0.041 & 3.757 \\
\hline 119.7 & 0.151 & 0.080 & 1.880 \\
\hline 137.5 & 0.115 & 0.050 & 2.289 \\
\hline 138.9 & 0.113 & 0.046 & 2.469 \\
\hline 126.6 & 0.136 & 0.052 & 2.645 \\
\hline 130.0 & 0.128 & 0.060 & 2.131 \\
\hline 158.4 & 0.086 & 0.049 & 1.740 \\
\hline & & & \\
\hline
\end{tabular}

Table 7: Flight test data points from Maiden 2 
These points were then plotted in two graphs: $C_{L}$ vs $C_{D}$ and $C_{L} / C_{D}$ vs airspeed. The primary unknown through the entire aircraft design was the $C_{D_{0}}$ value, which was required to generate a drag model of the aircraft. The initial assumption had been a value close to $C_{D_{0}}=0.03$, but as that was not known, multiple values of $C_{D_{0}}$ were plotted to show where the data points align in terms of the $\mathrm{C}_{\mathrm{D}_{0}}$ value. 


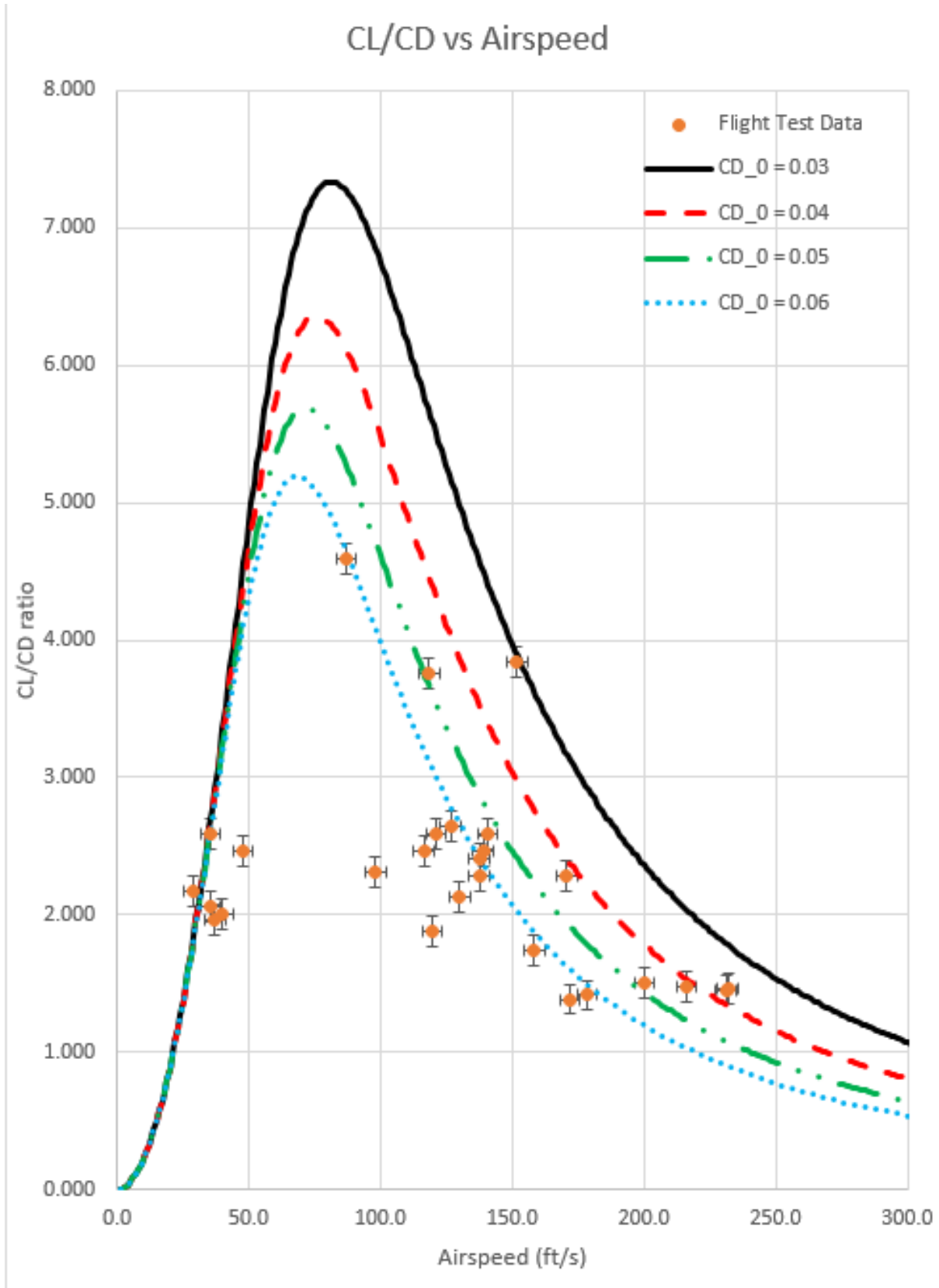

Figure 65: $C_{L} / C_{D}$ vs Airspeed with multiple values of $C_{D_{0}}$ from Maiden 2 


\section{CL vs CD (Assuming Steady, Level, Unaccelerated Flight)}

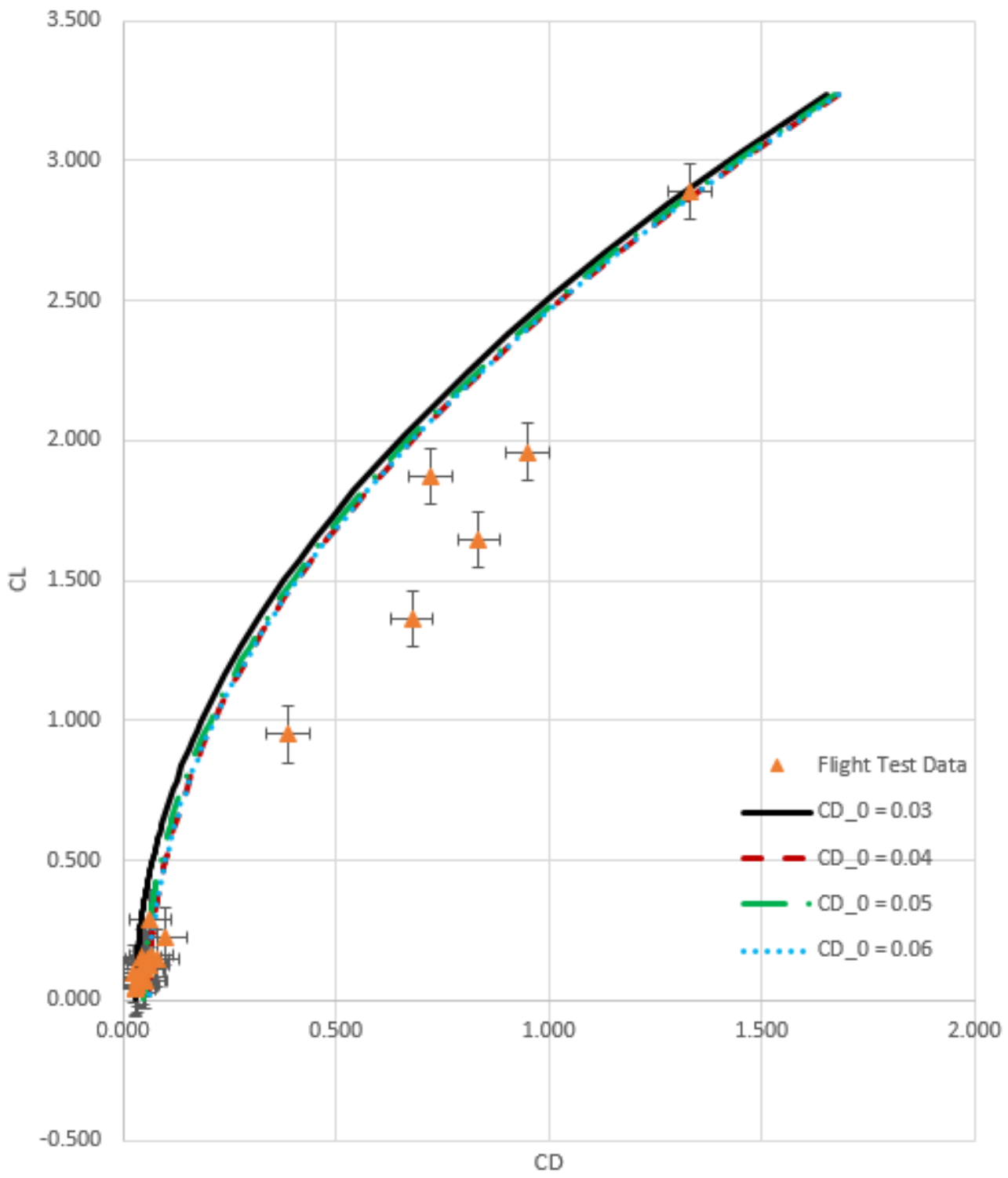

Figure 66: $C_{L}$ vs $C_{D}$ with multiple values of $C_{D_{0}}$ from Maiden 2

As can be seen from Figure 65, the max $C_{L} / C_{D}$ doesn't change much in the low speed regime, but at higher speeds the max values begin to drop. This agrees with the theory, as at low speed induced drag is most prevalent, while at higher speeds it's the parasite $\operatorname{drag}\left(C_{D_{0}}\right)$ that begins to be the dominant factor for $\mathrm{drag}^{30}$. From this flight testing, it appears the $\mathrm{C}_{\mathrm{D}_{0}}$ value 
is actually much closer to or potentially exceeding a value of $\mathrm{C}_{\mathrm{D}_{0}}=0.04$. More flight testing with a wider range of profiles will be conducted to investigate this further. Despite the plot from Figure 65 showing a strong correlation with $C_{D_{0}}$, the overall effect on the $C_{L}$ vs $C_{D}$ plot is minimal. This is due to the incremental increase in the $C_{D_{0}}$ value, which is simply a minor shift to the right on the x-axis. As whole, this plot shows that the theoretical drag model tends to hold fairly accurate, as has been verified by the flight testing.

Further flight tests will help to reveal whether the drag model is fully accurate or where modifications need to be made. As of now, the flight test data has shown that the previously used $C_{D_{0}}$ values of 0.03 are not entirely correct and that the flight test data shows it to more accurately be closer to 0.04 .

\subsection{Controls Analysis}

Before ever flying through a severe weather system, the goal was to estimate performance in high gust environments $(80 \mathrm{mph}+)$. The target observations are small tornadoes, such as EF-0 and EF-1 categories, which the National Weather Service defines as tornadoes producing 3 seconds wind gusts of 65-85 mph and 86-110 mph, respectively ${ }^{39}$. The Enhanced Fujita (EF) scale categorizes tornadoes based on their damage to structures, so these ranges are more driven towards gust loads as seen by structures ${ }^{26}$. These values could be much different at altitude, and since wind speeds at altitude have not yet been mapped for a tornado, there is much uncertainty in the wind speeds aloft even if the ground-based winds are known. These reasons were the driving force behind creating a set of aerodynamic matrices (as shown in Section 3.6.1) that could then be used to generate a gust model, giving some rudimentary insight into the potential behavior of the jet in various conditions. 


\subsubsection{Acquiring Coefficients}

Before acquiring any coefficients, it is useful to define the flight condition the analysis is over. For the purposes of this attempt, a steady and level cruise condition of $100 \mathrm{mph}$ at 1000 feet in altitude was chosen, as that is the speed for max endurance at a sufficient cruise altitude.

Before any of the equations in Figure 26 can be solved for, the coefficients for each force and moment must first be found. The most basic of the coefficients to solve for are the Coefficients of Lift and Drag. These were done using XFOIL, as it is commonly used as a 2-D solver for airfoils. As a reminder, the airfoil used in both the wings and winglets are biconvex with $10 \%$ thickness to length. Issues immediately arose from this analysis, as XFOIL's viscous solver does not permit for a sharp (no curvature, pointed) leading edge. As such, no values were able to be set allowing for viscous attempts. Various attempts, as can be seen in Appendix E, we made to get that data, and a few were able to be gathered, but the required airfoil geometry deviated greatly from the base biconvex airfoil. As such, the inviscid approach was used. This was not seen as an issue as the parasite drag (CD0) has been estimated from flight testing and other analyses conducted previously. Using this CD0 and having the lift curve slope, an approximation of total drag could be approximated. The results of the inviscid approach yielded a 2-D lift curve slop of $C_{l_{\alpha}}=.0738$ per degree of angle of attack.

This value was then used to approximate the lift curve slope of the entire aircraft. The equations used to do the conversions are shown below:

$$
C_{L_{\alpha}}=\frac{2 \pi A R}{2+\sqrt{4+\frac{A^{2} \beta^{2}}{\eta^{2}}\left(1+\frac{\tan ^{2} \Lambda_{\max t}}{\beta^{2}}\right)}}\left(\frac{S_{\text {exposed }}}{S_{\text {ref }}}\right)(F) \quad \text { EQ } 10
$$




$$
\begin{gathered}
\beta^{2}=1-M^{2} \\
\eta=\frac{C_{l_{\alpha}}}{2 \pi / \beta} \\
F=1.07\left(1+\frac{d}{b}\right)^{2}
\end{gathered}
$$

Where $C_{L_{\alpha}}$ is the wing (3-D) lift curve slope, $A R$ is the wings aspect ratio, $S_{\text {exposed }}$ is the exposed (wetted) surface area of the wing, $S_{r e f}$ is the reference wing planform area, $\Lambda_{\max t}$ is the sweep of the wing at the chord location where the airfoil is thickest, $\eta$ is the airfoil efficiency based on the airfoil lift curve slope $\left(C_{l_{\alpha}}\right)$ and Mach number $(M$, which is included by using the relationship $\beta$ ), and $F$ is the fuselage lift factor due to fuselage of diameter $d$ and total wingspan b. The result of these calculations was $C_{L_{\alpha}}=0.05$.

This value was then used to approximate the drag coefficient $\left(C_{D}\right)$. Since the 3-D coefficient of drag is closely tied to the coefficient of lift, the change in coefficient of drag due to changes in angle of attack, $C_{D_{\alpha}}$, was able to be found:

$$
\begin{gathered}
C_{D}=C_{D_{0}}+C_{D_{i}}=C_{D_{0}}+K C_{L}^{2}=C_{D_{0}}+\frac{C_{L}{ }^{2}}{\pi A R e} \\
e_{\text {swept wing }}=4.61\left(1-.045 A R^{0.68}\right)\left(\cos \Lambda_{L E}\right)^{0.15}-3.1
\end{gathered}
$$

Where the drag coefficient $\left(C_{D}\right)$ is a function of the parasite (zero lift) drag $\left(C_{D_{0}}\right)$ and the induced drag due to lift $\left(C_{D_{i}}\right)$, the induced drag is a function of the coefficient of lift $\left(C_{L}\right)$, the aspect ratio of the wing $(A R)$, and the Oswald efficiency factor $(e)$, and the efficiency factor for a swept wing (with wing sweep greater than $30^{\circ}$ ) is a function of aspect ratio $(A R)$, and the leading edge sweep $\left(\Lambda_{L E}\right)$. 
Using the 3-D lift curve slope equation to get a range of lift coefficients $\left(-5^{\circ}\right.$ to $\left.5^{\circ}\right)$, a range of drag coefficients could then be obtained using the 3-D drag coefficient equation. This range of drag coefficients over the specified range in angles of attack gave an effective change in drag coefficient due to angle of attack:

$$
C_{D_{\alpha}}=\frac{\Delta C_{D}}{\Delta \alpha}
$$

Which results in a $C_{D_{\alpha}}=.002$. While this value is used going forward, this is not assumed to be the true change in drag coefficient due to changes in angle of attack. To get a better estimate while still using the biconvex airfoil, a Computational Fluid Dynamics (CFD) study would need to be conducted to get a more accurate approximation on the viscous effects of the delta wing body, as there is an increased drag effect due to the fuselage shape.

The process for finding the lateral moment of inertia involved utilizing SolidWorks properties calculator. As the entire aircraft was modeled in the software with all parts to scale and in their respective locations, the inertia calculations were easily found, with the lateral component being $I_{y}=6.607 \mathrm{lbm} * \mathrm{ft}^{2}$.

It was at this stage that the data points gathered were then added to a table and compared against the other coefficients and values still needed to solve the matrix equations. A table that summarizes the values gathered so far versus what is still unfound in relation to Figure 26 is shown below: 


\begin{tabular}{|c|c|}
\hline Coefficeint & TIA/DB \\
\hline C_D_u & 0.034 \\
\hline C_D_0 & 0.031 \\
\hline Q (lbf/ft ${ }^{2}$ ) & 25.30 \\
\hline S (ft $\left.{ }^{2}\right)$ & 5.7 \\
\hline m (slugs) & 0.56 \\
\hline u0 (ft/s) & 146 \\
\hline C_D_alpha & 0.22 \\
\hline C_L_0 & -0.17 \\
\hline C_L_u & 0.082 \\
\hline C_L_alpha & 2.66 \\
\hline C_Z_alpha & \\
\hline C_bar & 1.55 \\
\hline C_Z_del_e & \\
\hline C_M_u & \\
\hline I_y (Ibm*ft*ft) & 6.607 \\
\hline C_M_alpha & \\
\hline C_M_del_e & \\
\hline C_M_alfa_dot & \\
\hline
\end{tabular}

Figure 67: Table of initial attempts at coefficients

From reviewing the processes required to find the remaining coefficients, major hurdles were encountered. Part of the data required is: horizontal tail area for $C_{m_{q}}, C_{L_{q}}, C_{Z_{q}}, C_{Z_{\dot{\alpha}}}$, distance of horizontal tail from CG for $C_{m_{q}}$ and $C_{m_{\dot{\alpha}}}$, and downwash angle of the horizontal tail for $C_{Z_{\dot{\alpha}}}$. Some of these, such as downwash angle and distance of the tail, can be approximated. However, there is not a proper way to account for a horizontal tail area, as the entire aircraft is one blended wing. Likewise, the presence of a thrust vectoring unit, which is the only form of yaw control and a significant portion of the pitch control, is directly tied to the turbine's throttle position. A change in throttle results in a change in the exhaust gas velocity, and this change in velocity generates a change in the effective forces imparted on the thrust vectoring unit. This coupling of throttle with lift and yaw, though integral to the successful operation of the aircraft, causes 
several key equations to fail. These equations are: $X_{\delta_{e}}, X_{\delta_{T}}, Z_{\delta_{e}}, Z_{\delta_{T}}, M_{\delta_{e}}, M_{\delta_{T}}$. These, coupled with the above mentioned equations that are not easily solved due to the lack of a horizontal tail, presented a major challenge to fully modeling the aerodynamics of the aircraft.

As these equations were not solvable by traditional means, there was no point in diving into this attempt further, as fully defining these control characteristics interactions at the required level are beyond the scope of the project. This will be left as a focus of future work. 


\section{CHAPTER V}

\section{CONCLUSIONS: FLIGHT AND SYSTEM REVIEW OF FINAL AIRCRAFT}

\subsection{TIA Compared to Other Severe Weather UAS}

Though TIA was designed with the intent on being a structural robust airframe whose goal is to penetrate tornadic systems for in-situ thermodynamic data acquisition, comparison to the current capabilities of other severe weather aircraft to the redesigned aircraft described here is needed.

Currently, UC Boulder's aircraft have been actively penetrating the gust fronts, bearing both winds and rain with minimal issues, with capabilities to stay on station for extended periods (longer than 1 hour). These aircraft are designed for long range and endurance for prolonged data collection in the gust fronts and downdrafts. As such, they feature high aspect ratio wings to achieve their performance metrics. Though there have been no major issues regarding winds encountered while aloft, these aircraft have the potential to experience issues when encountering a large asymmetric gusts (greater than $75 \mathrm{mph}$ ) rather than the typical straight line winds. This is due to the high aspect ratio wing having to resist bending under this gust loading, which could lead to structural fatigue or failure in these extreme cases. Another consideration for these aircraft is the transportation of the aircraft themselves. Though wings with large spans and high aspect ratios tend to have an increased aerodynamic performance, this also requires the aircraft to be 
taken down and packed away for all modes of transportation. Though this can decrease the time needed to replace faulty hardware or fatigued structure, it also increases the setup and takedown time as compared to an all-in-one system.

TIA, having a different target of operation, was designed to withstand these large gusts while maintaining structural integrity. Though the specific target and the approaches taken to achieve it have resulted in a large reduction in range and endurance performance metrics as compared to the other aircraft, the aircraft's robustness allows it to operate in environments that could potential damage or destroy traditional high aspect ratio airframes. As the airframe is one solid body (aside from winglets), there is no takedown or setup required. The aircraft can be taken out of the transport vehicle, fueled up, and launched. From flight testing, this total time before takeoff has been demonstrated to be achievable in under 5 minutes, including for takeoffs after landings requiring a refuel and battery swap. This allows the aircraft to fly for nearly an hour in duration with a total time on the ground of less than 10 minutes, which helps supplement the reduced endurance compared to the other long endurance aircraft.

\begin{tabular}{|l|c|c|c|c|c|c|}
\cline { 2 - 7 } \multicolumn{1}{c|}{} & TIA & Maria & Tempest & Tempest & Mistral & RAAVEN \\
\hline GTOW (lbs) & 18 & 35 & 15 & 20 & 40 & 4 \\
\hline Propulsion Type & $\begin{array}{c}\text { Diesel } \\
\text { Turbojet }\end{array}$ & $\begin{array}{c}\text { Electric or } \\
\text { Gas Prop }\end{array}$ & Electric Prop & $\begin{array}{c}\text { Dual Electric } \\
\text { Prop }\end{array}$ & $\begin{array}{c}\text { Dual } \\
\text { Electric } \\
\text { Prop }\end{array}$ & $\begin{array}{c}\text { Electric } \\
\text { Prop }\end{array}$ \\
\hline Top Speed $(\mathrm{mph})$ & 160 & 110 & 78 & 95 & 100 & 80 \\
\hline Endurance $(\mathrm{min})$ & 20 & - & 180 & 180 & 180 & 120 \\
\hline Rain Tier & 3 & 4 & 4 & 4 & 4 & 4 \\
\hline Wind Tier & 5 & 5 & 5 & 5 & 5 & 4 \\
\hline Gust Tier & 5 & 5 & 4 & 4 & 4 & 3 \\
\hline Primary Objective & $\begin{array}{c}\text { Targeted } \\
\text { Intercept }\end{array}$ & \multicolumn{7}{|c|}{ Long endurance monitoring } & \\
\hline
\end{tabular}

Figure 68: TIA compared to other severe weather UAS

Of note, though much comparison is shown between TIA and other active severe weather aircraft, TIA is best implemented as a compliment to these UAS, not as a replacement of any 
form. Aircraft such as Tempest are required for long endurance data collection of moving systems, while aircraft such as TIA are required to penetrate specific targets that would otherwise bring great risk to standard aircraft, such as the spin-up of a funnel cloud, which is a precursor to a tornado. The higher top speed metric (160 mph compared to $100 \mathrm{mph}$ ) allows for rapid interception, with a low cruise speed capable to gather the same data. The low aspect ratio wing enables the aircraft to better penetrate large gusts with reduced risk of structural failure.

\subsection{Summary of Aircraft Capabilities}

The end result of all the testing detailed in the previous sections is an aircraft that flies very reliably, and after turning the autopilot handles very well in all flight modes. After the redesign, the aircraft went from a 7.5 pound base weight, to 11 pounds in the prototype phase, before reaching a final weight of 18 pounds for TIA. The aircraft has reached sustained speeds of $160 \mathrm{mph}$ on the high end (13 minute endurance, 34 mile 1-way range), a cruise speed of $100 \mathrm{mph}$ (20 minute endurance, 33 mile 1-way range), and a minimum safe steady level speed of $30 \mathrm{mph}$.

If conducting a high angle of attack landing, that minimum airspeed drops dramatically to $12 \mathrm{mph}$ or lower, depending on ambient winds conditions. These metrics allow for a structurally robust aircraft to reach a target environment quickly traveling at top speed, loiter on station for a prolonged period to gather data, then return back to launch or wherever the personnel still are to recover the data and aircraft. By utilizing the high angle of attack landings, the aircraft can land on many surfaces while taking little to no damage (grass strips, dirt roads, fields, pavement, etc.). Having a high top speed threshold allows for the observation of system with high sustained winds $(80 \mathrm{mph}+)$, which is not typical of UAS to operate in. However, to accomplish these feats comes at the cost of reduced endurance and range as compared to most atmospheric sensing UAS. 
The presence of water shielding additives in the nose and on the hatches help ensure the aircraft can survive even in moderate rain, but there is a limit to the amount of precipitation the jet can operate in. Heavier rainfall rates correspond to less air available for the turbine to draw in, creating a currently unknown but very critical limit for the aircraft in its current configuration. Knowing at what point the turbine fails due to the flame in the combustion chamber being extinguished (or any other potential yet unknown error source) is pivotal to the success of this craft in heavy rain. Though the small turbines used by TIA have not undergone water ingestion testing, this will be one focus of future work, as there is currently no data on how sUAS turbines react to varying degrees of water ingestion.



Figure 69: Graphical transition from prototype to final product. Blue components were removed or upgraded, Orange components were added.

The aircraft has been full outfitted with an autopilot for long range, beyond visual line of sight flights, as well as long range antennas on all systems (RC, FPV, autopilot) to maintain control over the aircraft even as it goes well beyond line of sight ( $3+$ miles), depending on the terrain and how much obstructions the RF signals must pass through. With the autopilot' GPS system, "geo-fences" can be established to limit where the aircraft both is and isn't permitted to fly. These systems also allow for various failsafe options to be enabled in the event the aircraft 
loses RC or telemetry link or the aircraft is behaving erratically, which introduces a heightened level of safety when operating near in an area that could cause potential damage to personal property.

Though the aircraft began as a hand-launched system, the roof-mounted car launcher system has shown great promise in launching the full jet-powered aircraft. This system was dialed in over multiple trials of various types and weights of aircraft, and using these test aircraft allowed for the autopilot to take control starting before the release from the launcher directly into an auto mission without any input required from the pilot. The electric version of TIA has launched multiple times, but from those launches it was noted more flight testing at different static angles and speeds would be needed before launching the jet-powered airframe to reduce potential damage to the airframe from a failed launch. By fine tuning this approach, the jetpowered UAS will be capable of launching from any area that the ground launch vehicle can achieve speeds of at least $35 \mathrm{mph}$ while operable from even an inexperienced ground crew. The requirement that remains constant is a skilled pilot for landing.

\subsection{Continuing Progress}

Though TIA has been demonstrated to be field-ready, it has not launched off the launcher, which is the target method for its ease of operation and reliability. However, this reliability must be built up for this aircraft itself. To accomplish this, a test matrix of car launches using Beast (TIA prototype) must be completed. This involves launching at different speeds (35$50 \mathrm{mph}, 5 \mathrm{mph}$ increments) and static launch angles $\left(0^{\circ}-6^{\circ}, 2^{\circ}\right.$ increments $)$. The current launch speeds have been $35 \mathrm{mph}$, but seeing the influence to faster speeds is required in the event crosswind conditions are experienced. The current launch angle is $0^{\circ}$, but increasing that angle is needed in conjunction with the changing launch speeds to determine if the aircraft performs better 
in one condition versus another. The resulting data sets will drive the launch condition of TIA's first launch.

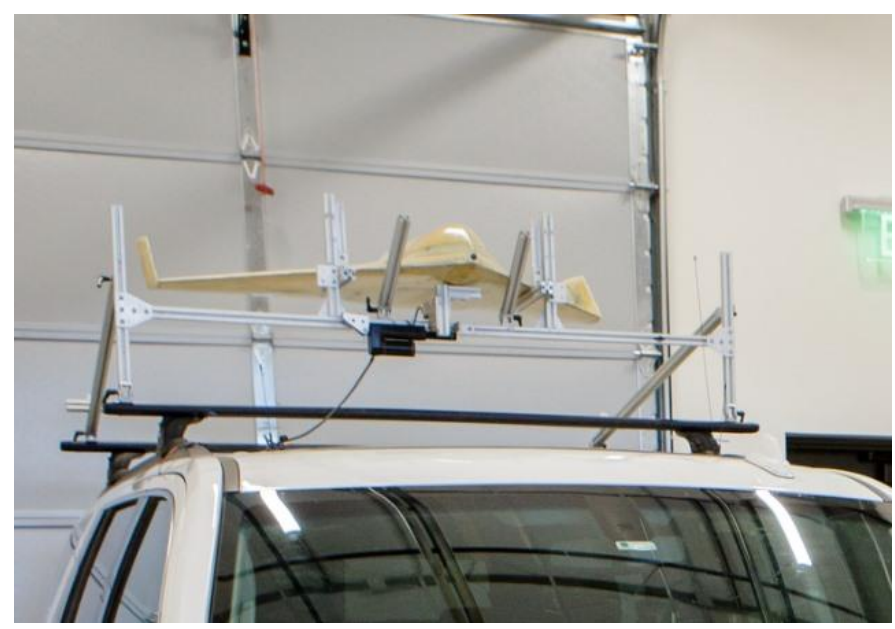

Figure 70: TIA mounted on launcher for sizing

Once the aircraft is able to be launched from the launcher, the next step is to integrate the weather sensors into the Port winglet. This will be done using a pitot tube that protrudes from the winglet, allowing for forced aspiration of the sensors inside the winglet. To prevent the board from frying in the event of precipitation, the digital sensors will be wired via extension to the primary board, which will be placed in the Rear Avionics Bay on the Port side near the Video Transmitter. When the sensors are calibrated and validated to be gathering accurate data, the entire system will be ready for atmospheric data collection.

\subsection{Future Work}

Beyond the full completion of the aircraft, a renewed visit to the aerodynamic model is necessary to both predict the performance of the aircraft before penetrating large gusts (60 $\mathrm{mph}+$ ), but also to compare the predicted performance to the actual performance. The aerodynamic model was attempted to glean insight from, longitudinal, lateral, and gust controls 
analyses, but due to the tailless delta wing nature of the jet and the addition of a thrust vectoring system, that approach was ultimately ceased. Though the aircraft has not flown in high winds $(50+\mathrm{mph})$ and there is not a valid aerodynamic control model to approximate the response of the system, the aircraft has flown in sustained $25 \mathrm{mph}$ winds, with some cases the winds reaching gusts of $33 \mathrm{mph}$. Though these cases tend to complicate the high angle of attack landings if these same winds are variable at ground level (stable winds make landings easier), a semi-conventional landing can be attempted if this wind behavior is observed. If winds are relatively constant in intensity, a high angle of attack landing can still be achieved, as has been demonstrated by previous flight tests.

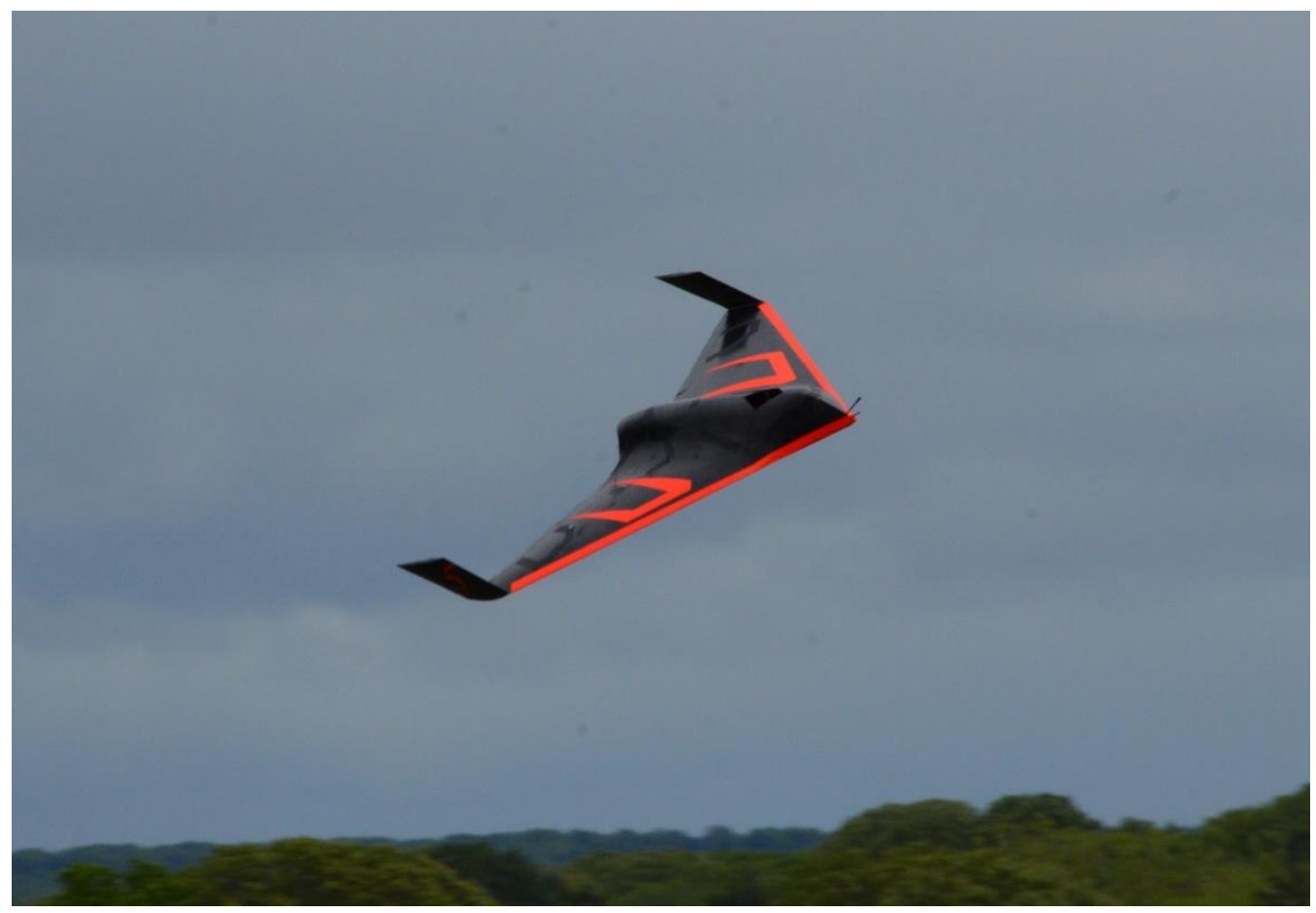

Figure 71: TIA prototype in flight 


\section{REFERENCES}

1 "NWS GPRA Metrics," National Weather Service, https://verification.nws.noaa.gov/services/gpra/NWS_GPRA_Metrics.pdf, 2018.

2 "Understand Tornado Alerts," National Weather Service, https://www.weather.gov/safety/tornado-ww

${ }^{3}$ Elston, J., Argrow, B., Houston, A. and Frew, E., "Design and Validation of a system for Targeted Observations of Tornadic Supercells Using Unmanned Aircraft," Institute for Electrical and Electronics Engineers, Intelligent Robots and Systems. Oct 18, 2010.

${ }^{4}$ Brock, F., Crawford, K., “The Oklahoma Mesonet: A Technical Overview," American Meteorological Society, 1995.

5 "NWS Radiosonde Observations-Factsheet," National Weather Service, http://www.erh.noaa.gov/gyx/weather balloons.htm, 2001.

6 "NEXRAD," National Weather Service, https://www.ncdc.noaa.gov/data-access/radardata/nexrad

${ }^{7}$ Grunbaum, M., "Weather-Studying Warthog: A Fixed-Up A-10 Will Fly Into Thunderstorms," Popular Mechanics, 2011.

8 "NRL's P-3 Aircraft Support Project to Study Tropical Cyclones," http://www.nrl.navy.mil/media/news-releases, 2008.

${ }^{9}$ Elston, J., and Frew, E., "Unmanned Aircraft Guidance for Penetration for Pre-Tornadic Storms," AIAA Paper 6513, AIAA Guidance, Navigation and Control Conference and Exhibit, Honolulu, HA, 1821 August 2008.

${ }^{10}$ Elston, J. S., Roadman, J., Stachura, M., Argrow, B., Houston, A., \& Frew, E. (2011). The tempest unmanned aircraft system for in situ observations of tornadic supercells: Design and VORTEX2 flight results. Journal of Field Robotics, 28(4), 461-483. https://doi.org/10.1002/rob.20394

${ }^{11}$ Alyssa, A S. and Jacob, J. D., 2014, "Design of a Severe Storm Research UAS," 53rd AIAA Aerospace Sciences Meeting, Orlando, FL, Jan., 2014.

${ }^{12}$ Houston, A. L., Argrow, B., Elston, J., Lahowetz, J., Frew, E. W., \& Kennedy, P. C. (2012). The Collaborative Colorado-Nebraska Unmanned Aircraft System Experiment. Bulletin of the American Meteorological Society, 93(1), 39-54.

https://doi.org/10.1175/2011bams3073.1 
${ }^{13}$ Elston, J., Argrow, B., Frew, E., Houston, A., \& Straka, J. (2011). Evaluation of Unmanned Aircraft Systems for Severe Storm Sampling Using Hardware-in-the-Loop Simulations. Journal of Aerospace Computing, Information, and Communication, 8(9), 269-294. https://doi.org/10.2514/1.53737

${ }^{14}$ Houston, Adam \& Argrow, B. \& Frew, E.. (2010). Observing Supercells with Unmanned Aircraft: Results from the UAS Component of VORTEX2. AGU Fall Meeting Abstracts.

${ }^{15}$ University of Nebraska-Lincoln. (2009). Unmanned Aircraft System \& Severe Storms Research Group (USSRG) | Nebraska. Retrieved from Unl.edu website: https://ussrg.unl.edu/

${ }^{16}$ TORUS: Targeted Observations by Radars and UAS of Supercells. (2015). Retrieved from NOAA National Severe Storms Laboratory website: https://www.nssl.noaa.gov/projects/torus/

${ }^{17}$ Unmanned Systems. (2015, April 9). Retrieved from Research and Engineering Center for Unmanned Vehicles website: https://www.colorado.edu/recuv/facilities/unmannedsystems

${ }^{18}$ VORTEX @ NSSL. (2015). Retrieved from NOAA National Severe Storms Laboratory website: https://www.nssl.noaa.gov/projects/vortex/

${ }^{19}$ Elston, J. S., Roadman, J., Stachura, M., Argrow, B., Houston, A., \& Frew, E. (2011). The tempest unmanned aircraft system for in situ observations of tornadic supercells: Design and VORTEX2 flight results. Journal of Field Robotics, 28(4), 461-483. https://doi.org/10.1002/rob.20394

${ }^{20}$ The TORUS Project Will Use Drones to Study Tornados. (2019, April 19). Retrieved from Integrated Remote and In-Situ Sensing website: https://www.colorado.edu/iriss/torus

${ }^{21}$ UAS Equipment. (2018, June 11). Retrieved from Integrated Remote and In-Situ Sensing website: https://www.colorado.edu/iriss/content/equipment

${ }^{22}$ Meet the RAAVEN drone that gathers data from the storms. (2019, July). Retrieved from College of Engineering \& Applied Science website: https://www.colorado.edu/engineering/2019/07/01/meet-raaven-drone-gathers-datastorms

${ }^{23}$ The Sirens Project - UAV Tornado Research. (2016, December 2). Retrieved from Kickstarter website: https://www.kickstarter.com/projects/1517270439/the-sirens-project-uavtornado-research

${ }^{24}$ Oglesby, B. and Arena, A. S., 2016, 'Experimental Study of a Small Turbojet for Use in an Unmanned Aircraft System” Oklahoma State University, Stillwater, OK, 2016.

${ }^{25}$ Damaging Winds Basics. (2015). Retrieved from NOAA National Severe Storms Laboratory website: https://www.nssl.noaa.gov/education/svrwx101/wind/

${ }^{26}$ US Department of Commerce, NOAA, National Weather Service. (2019). The Enhanced Fujita Scale (EF Scale). Retrieved from Weather.gov website: https://www.weather.gov/oun/efscale

${ }^{27}$ DJI M100 specs https://www.dji.com/matrice100/info

${ }^{28}$ DJI M200 specs https://www.dii.com/matrice-200-series/info 
${ }^{29}$ Azartash-Namin, K. (2017). EVALUATION OF LOW-COST MULTI-HOLE PROBES FOR

ATMOSPHERIC BOUNDARY LAYER INVESTIGATION. Retrieved from

https://shareok.org/bitstream/handle/11244/299672/AzartashNamin_okstate_0664M_ 15384.pdf?sequence $=1$

${ }^{30}$ Daniel Raymer, Aircraft Design: A Conceptual Approach, Fifth Edition, AIAA, 2012.

${ }^{31}$ Commercial Operations Branch - Part 107 UAS Operations. (2018, September 27). Retrieved from Faa.gov website:

https://www.faa.gov/about/office_org/headquarters_offices/avs/offices/afx/afs/afs800/afs 820/part107_oper/

${ }^{32}$ Schematic of Vortex Lift on slender delta wing. XXXX Vortex Lift image Rahman, Hammad \& Khushnood, Shahab \& Raza, A. \& Ahmad, Kamran. (2013). Experimental and computational investigation of delta wing aerodynamics. 203-208.

10.1109/IBCAST.2013.6512155.

${ }^{33}$ Speedfest VII Alpha Class Rules, http://speedfest.okstate.edu/Speedfest VII/Speedfest VII Rules Alpha.html

${ }^{34}$ Kakkar, Baba, "Experimental Investigations of Optimal Aerodynamics of a Flying Wing UAV", University of Bath, Bath, United Kingdom, 2016.

35 UAV Factory - Unmanned Platforms and Subsystems. (2019). Retrieved from Uavfactory.com website: https://www.uavfactory.com/product/47

${ }^{36}$ Drag Coefficient, https://www.engineeringtoolbox.com/drag-coefficient-d_627.html

${ }^{37} 8020$ deflection calculator, https://8020.net/deflection-calculator

${ }^{38}$ Barbieri, L., Kral, S., Bailey, S., Frazier, A., Jacob, J., Reuder, et.al. (2019). Intercomparison of Small Unmanned Aircraft System (sUAS) Measurements for Atmospheric Science during the LAPSE-RATE Campaign. Sensors, 19(9), 2179. https://doi.org/10.3390/s19092179

${ }^{39}$ Severe Weather Awareness - Tornado Classification and Safety. Retrieved from Weather.gov website: https://www.weather.gov/mkx/taw-tornado_classification_safety

${ }^{40}$ Rain - AMS Glossary. (2012). Retrieved from Ametsoc.org website: http://glossary.ametsoc.org/wiki/Rain

${ }^{41}$ Motor-induced torque effect, FAA handbook, https://www.faa.gov/regulations_policies/handbooks_manuals/aviation/phak/media/07_p hak ch5.pdf

${ }^{42}$ Technique - Left Turning Tendencies. (2014, May 10). Retrieved from Aopa.org website: https://www.aopa.org/news-and-media/all-news/2014/october/flight-trainingmagazine/technique--left-turning-tendencies

${ }^{43}$ Robert Nelson, "Flight Stability and Automatic Control", Second Edition, McGraw-Hill, 1998.

${ }^{44}$ Hunt, D. and Arena, A. S., 2012, 'Structural Design and Fabrication Techniques of Composite Unmanned Aerial Vehicles" Oklahoma State University, Stillwater, OK, 2012.

${ }^{45}$ Ready Made RC, LLC. (2019). Skywalker X8 Kit 2120mm EPO FPV Wing. Retrieved from Readymaderc.com website: https://www.readymaderc.com/products/details/skywalkermodel-x8-black-wing 
${ }^{46}$ D. Moerder and A. J. Calise, "Convergence of a Numerical Algorithm for Calculating Optimal Output Feedback Gains," IEEE Transactions on Automatic Control, vol. 30, no. 9, pp. 900-903, 1985.

${ }^{47}$ A. J. Calise and D. D. Moerder, "Optimal Output Feedback Design of Systems with IllConditioned Dynamics," Automatica, vol. 21, no. 3, pp. 271-276, 1985. 


\section{APPENDICES}

\section{APPENDIX A: AVIONICS SETTINGS}

Important Pixhawk parameters for turbine use with thrust vectoring - Pitch to Servo commands

- PTCH2SRV_RLL - gain added to pitch to keep aircraft from ascending/descending during turns (increase to increase ascension, decrease to improve descension)

- TECS_RLL2THR - gain to control additional throttle added during a turn to maintain energy (i.e. speed) to overcome extra drag. Not used, but could be in the future

- $\quad$ Limit RLL2SRV_IMAX from +- $30^{\circ}$ to $+-5^{\circ}$ : when coming out of banks, can keep the igains offset from the trim condition, so the degree it can control was reduced

Important Pixhawk parameters for Auto launching

- TKOFF_THR_MINSPD - min GPS speed to arm motors and unsuppress throttle for auto takeoff

- $\quad$ TKOFF_THR_MINACC - min acceleration autopilot registers before arming motors, for catapult launches

- TKOFF_THR_DELAY - delay time (in 0.1 second increments) before motors actually start up after being armed by either of the two above settings.

Reduced i-gain in roll to prevent over-rotation during banks during a prolonged flight 
Appendix A2: PWM Signal Tracking Through All Avionics Systems

\begin{tabular}{|c|c|c|c|c|c|c|c|}
\hline Test & Measurand & Center & $50 \%$ Left & $100 \%$ Left & $\begin{array}{c}50 \% \text { Diff } \\
\text { from Center }\end{array}$ & $\begin{array}{l}100 \% \text { Diff } \\
\text { from Center }\end{array}$ & Comments \\
\hline \multirow{2}{*}{$\begin{array}{c}\text { Servo } \\
\text { Programmer }\end{array}$} & Angle & 90 & 120 & 150 & 30 & 60 & \multirow{2}{*}{$\begin{array}{l}\text { Pure signal movement from a programmer (max range of } \\
\text { servo) }\end{array}$} \\
\hline & PWM & 1500 & 1200 & 900 & -300 & -600 & \\
\hline Pure RC & Angle & 88 & 109 & 131 & 21 & 43 & $\begin{array}{l}\text { Movement via signals directly from RC } \\
\text { transmitter/receiver }\end{array}$ \\
\hline \multirow{2}{*}{$\begin{array}{c}\mathrm{RC} \rightarrow \text { Aura } \\
\text { (Stock Settings) }\end{array}$} & Angle & 89 & 101 & 115 & 12 & 26 & \multirow{2}{*}{$\begin{array}{l}\text { Servo signals passing from RC receiver through Aura } 8 \\
\text { with default settings to the servo. Large reduction in } \\
\text { angular deflection at stock settings. }\end{array}$} \\
\hline & Aura PWM & 1501 & 1378 & 1241 & -123 & -260 & \\
\hline \multirow{2}{*}{$\begin{array}{c}\text { RC } \rightarrow \text { Aura } \\
\text { (Output Tune) }\end{array}$} & Angle & 89 & 101 & 115 & 12 & 26 & \multirow{2}{*}{$\begin{array}{l}\text { Output tune doesn't seem to change anything of } \\
\text { relevance }\end{array}$} \\
\hline & Aura PWM & 1501 & 1379 & 1241 & -122 & -260 & \\
\hline \multirow{2}{*}{$\begin{array}{l}\text { RC } \rightarrow \text { Aura } \\
\text { (Pulse Set) } \\
\end{array}$} & Angle & 89 & 108 & 128 & 19 & 39 & \multirow{2}{*}{$\begin{array}{l}\text { Changing Pulse Settings seem to allow for larger output } \\
\text { for a given percent RC input }\end{array}$} \\
\hline & Aura PWM & 1501 & 1317 & 1112 & -184 & -389 & \\
\hline \multirow{2}{*}{$\begin{array}{l}\text { RC } \rightarrow \text { Aura } \\
\text { (TIA Settings) }\end{array}$} & Angle & 89 & 108 & 128 & 19 & 39 & \multirow{2}{*}{$\begin{array}{l}\text { Combining the two shows the Pulse setting is the } \\
\text { dominant factor with Output Tune not having a } \\
\text { noticeable effect }\end{array}$} \\
\hline & Aura PWM & 1501 & 1316 & 1112 & -185 & -389 & \\
\hline \multirow{3}{*}{$\begin{array}{c}\text { RC } \rightarrow \text { Pix } \\
\text { (Stock Params, } \\
\text { Aileron) }\end{array}$} & Angle & 89 & 109 & 130 & 20 & 41 & \multirow{3}{*}{$\begin{array}{l}\text { Follows the Pure RC output very closely, with margin of } \\
\text { error causing the only noted deviation }\end{array}$} \\
\hline & Pix PWM In & 1518 & 1307 & 1099 & -211 & -419 & \\
\hline & Pix PWM Out & 1500 & 1299 & 1100 & -201 & -400 & \\
\hline \multirow{3}{*}{\begin{tabular}{|c|}
$\mathrm{RC} \rightarrow$ Pix \\
(Increased \\
Output, Aileron)
\end{tabular}} & Angle & 89 & 120 & 150 & 31 & 61 & \multirow{3}{*}{$\begin{array}{l}\text { Increasing Output PWM to match servo programmer's } \\
\text { range resulted in angle matching as well. }\end{array}$} \\
\hline & Pix PWM In & 1518 & 1307 & 1099 & -211 & -419 & \\
\hline & Pix PWM Out & 1500 & 1203 & 900 & -297 & -600 & \\
\hline \multirow{3}{*}{$\begin{array}{c}\text { RC } \rightarrow \text { Pix } \\
\text { (Increased } \\
\text { Output, RC1) }\end{array}$} & Angle & 88 & 108 & 130 & 20 & 42 & \multirow{3}{*}{$\begin{array}{l}\text { Changing the output channel from Aileron (modified } \\
\text { signal) to RC1 (pure RC pass-through) allowed for angles } \\
\text { similar to pure RC but less accurate than using the } \\
\text { modified signal channel }\end{array}$} \\
\hline & Pix PWM in & 1518 & 1305 & 1099 & -213 & -419 & \\
\hline & Pix PWM Out & 1518 & 1305 & 1099 & -213 & -419 & \\
\hline \multirow{3}{*}{$\begin{array}{l}\text { RC } \rightarrow \text { Pix } \\
\text { (TIA Params, } \\
\text { Aileron) }\end{array}$} & Angle & 89 & 105 & 121 & 16 & 32 & \multirow{3}{*}{$\begin{array}{l}\text { This was a test to see how TIA's settings compare to the } \\
\text { other permutations. No information is really gathered } \\
\text { from this as the TIA's servos are programmed and this } \\
\text { test servo is set at all stock parameters. }\end{array}$} \\
\hline & Pix PWM In & 1518 & 1309 & 1099 & -209 & -419 & \\
\hline & Pix PWM Out & 1500 & 1310 & 1183 & -190 & -317 & \\
\hline \multirow{3}{*}{$\begin{array}{c}\text { RC } \rightarrow \text { Pix } \rightarrow \text { Aura } \\
\text { (All Stock } \\
\text { Params) }\end{array}$} & Angle & 90 & 104 & 117 & 14 & 27 & \multirow{3}{*}{$\begin{array}{l}\text { Appears that running all default params results in a } 55 \% \\
\text { reduction in angle at a given PWM compared to the } \\
\text { programmer and a } 37 \% \text { reduction compared to the Pure } \\
\text { RC test. Aura } 8 \text { is the limiter as seen from its independent } \\
\text { tests }\end{array}$} \\
\hline & Pix PWM Out & 1500 & 1300 & 1100 & -200 & -400 & \\
\hline & Aura PWM & 1490 & 1359 & 1219 & -131 & -271 & \\
\hline \multirow{3}{*}{$\begin{array}{c}\text { RC } \rightarrow \text { Pix } \rightarrow \text { Aura } \\
\text { (Aura stock, Pix } \\
\text { Output) }\end{array}$} & Angle & 90 & 110 & 132 & 20 & 42 & \multirow{3}{*}{$\begin{array}{l}\text { Increasing Pixhawk output scale while keeping Aura at } \\
\text { stock values very nearly matches the pure RC tests }\end{array}$} \\
\hline & Pix PWM Out & 1500 & 1198 & 900 & -302 & -600 & \\
\hline & Aura PWM & 1490 & 1289 & 1080 & -201 & -410 & \\
\hline \multirow{3}{*}{$\begin{array}{c}\text { RC } \rightarrow \text { Pix } \rightarrow \text { Aura } \\
\text { (Pix stock, Aura } \\
\text { Pulse) }\end{array}$} & Angle & 90 & 110 & 132 & 20 & 42 & \multirow{3}{*}{$\begin{array}{l}\text { Increasing Aura's Pulse output settings while keeping all } \\
\text { Pixhawk values default also very nearly matches pure RC } \\
\text { tests. Could be a doubling effect seen once they are } \\
\text { combined. }\end{array}$} \\
\hline & Pix PWM Out & 1500 & 1301 & 1100 & -199 & -400 & \\
\hline & Aura PWM & 1486 & 1290 & 1078 & -196 & -408 & \\
\hline \multirow{3}{*}{$\begin{array}{c}\text { RC } \rightarrow \text { Pix } \rightarrow \text { Aura } \\
\text { (Pix TIA params, } \\
\text { Aura stock) }\end{array}$} & Angle & 90 & 101 & 111 & 11 & 21 & \multirow{3}{*}{$\begin{array}{l}\text { Using the TIA Pixhawk params and a stock Aura, there is } \\
\text { reduced output, lowest of all the testing. Aura } 8 \text { output is } \\
\text { a driving force behind this, as anytime the Aura output is } \\
\text { low, overall output is low. This makes sense as it is } \\
\text { directly fed to servos }\end{array}$} \\
\hline & Pix PWM Out & 1497 & 1338 & 1183 & -159 & -314 & \\
\hline & Aura PWM & 1490 & 1389 & 1279 & -101 & -211 & \\
\hline & Angle & 89 & 114 & 142 & 25 & 53 & Combining the Pixhawk and Aura 8 parameters (as \\
\hline (Direct From TIA & Pix PWM Out & 1500 & 1341 & 1183 & -159 & -317 & $\begin{array}{l}\text { measured from TIA integrated system with same test } \\
\text { servo) resulted in values slightly lower than from the }\end{array}$ \\
\hline & Aura PWM & 1498 & 1244 & 995 & -254 & -503 & output from the programmer. \\
\hline
\end{tabular}

Table 8: Full table of PWM signal pass-through of all avionics systems 


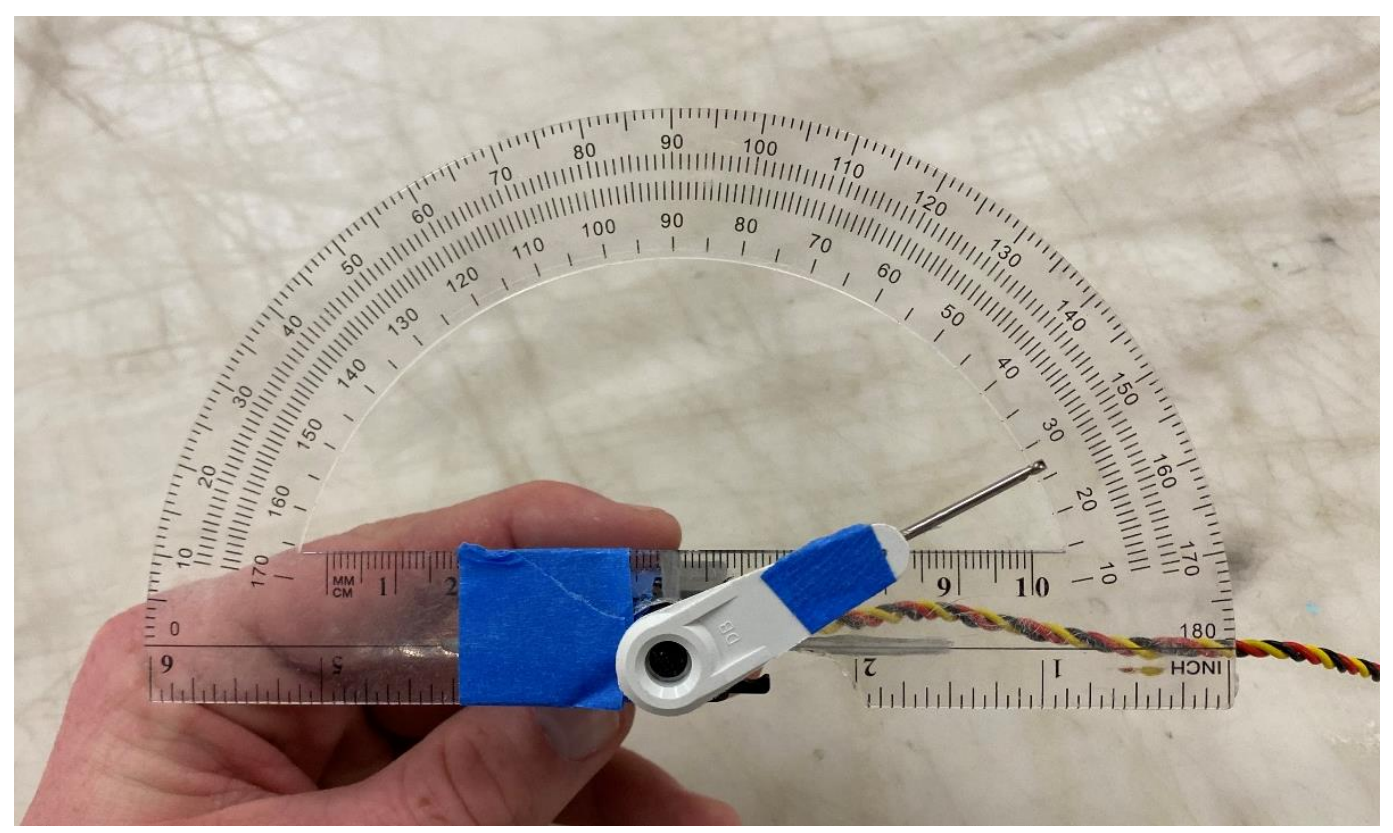

Figure 72: Used to measure angular deflections recorded in Table 8

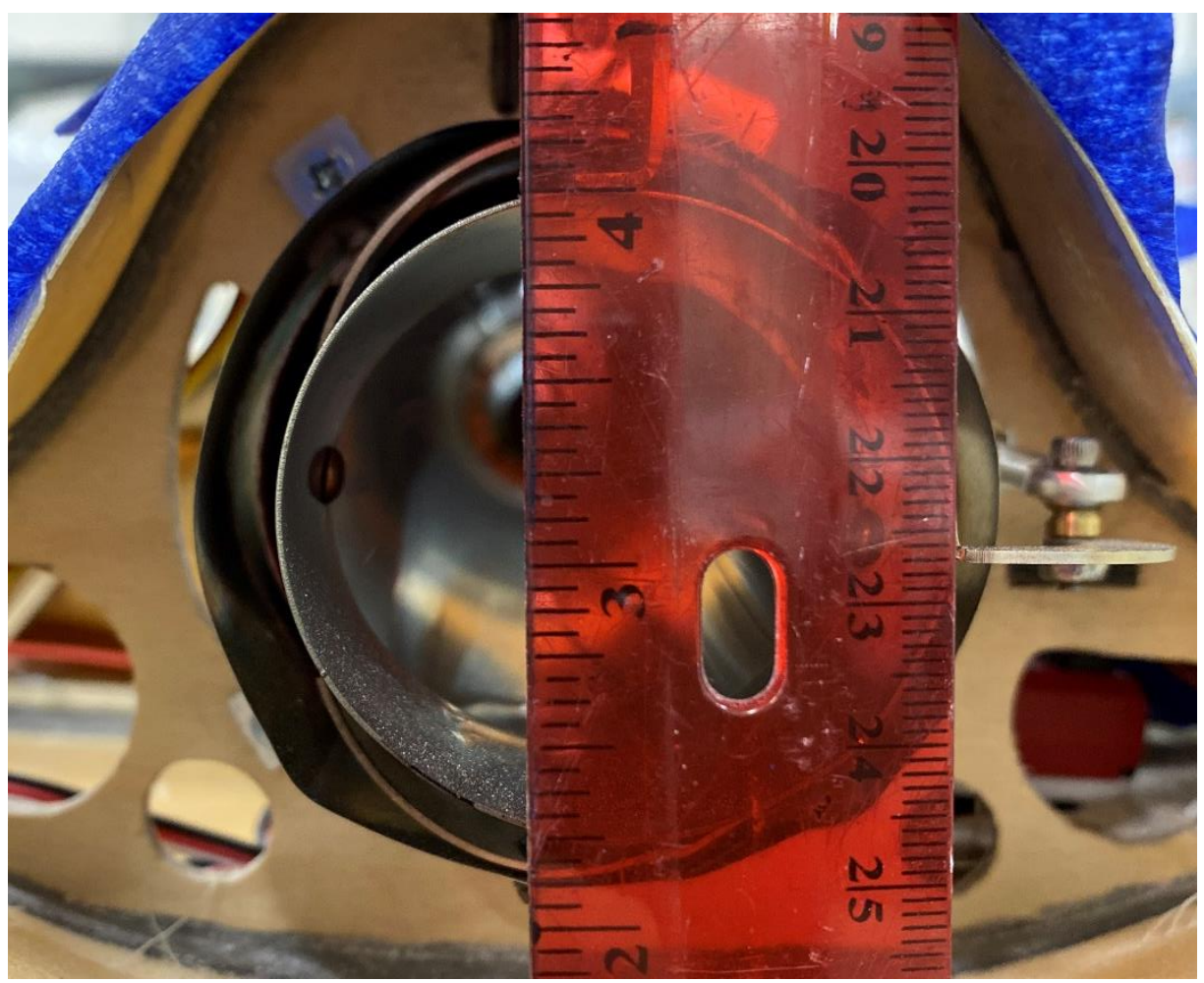

Figure 73: Used to measure pitch thrust vectoring angular deflections recorded in Table 9 


\begin{tabular}{|c|c|}
\hline \multicolumn{2}{|c|}{ Pitch TV Servo } \\
\hline PWM & Angle $\left({ }^{\circ}\right)$ \\
\hline 1139 & -7.9 \\
\hline 1150 & -7.1 \\
\hline 1200 & -6.3 \\
\hline 1250 & -5.6 \\
\hline 1300 & -4.8 \\
\hline 1350 & -4.0 \\
\hline 1400 & -3.2 \\
\hline 1450 & -1.6 \\
\hline 1500 & 0.0 \\
\hline 1550 & 1.6 \\
\hline 1600 & 3.2 \\
\hline 1650 & 4.8 \\
\hline 1700 & 6.3 \\
\hline 1750 & 7.9 \\
\hline 1800 & 9.5 \\
\hline 1850 & 11.0 \\
\hline 1900 & 12.5 \\
\hline 1937 & 12.5 \\
\hline
\end{tabular}

Table 9: Pitch thrust vectoring deflection at each PWM signal value 


\section{APPENDIX B: PROPULSION DATA}

The first part of this section describes the initial turbine inlet sizing for the prototype's Kingtech K45G1, but a similar process was conducted to size the inlet for TIA. Prototype (Diamondback) had an inlet area of $12 \mathrm{in}^{2}$ while TIA has an inlet area of $20 \mathrm{in}^{2}$.
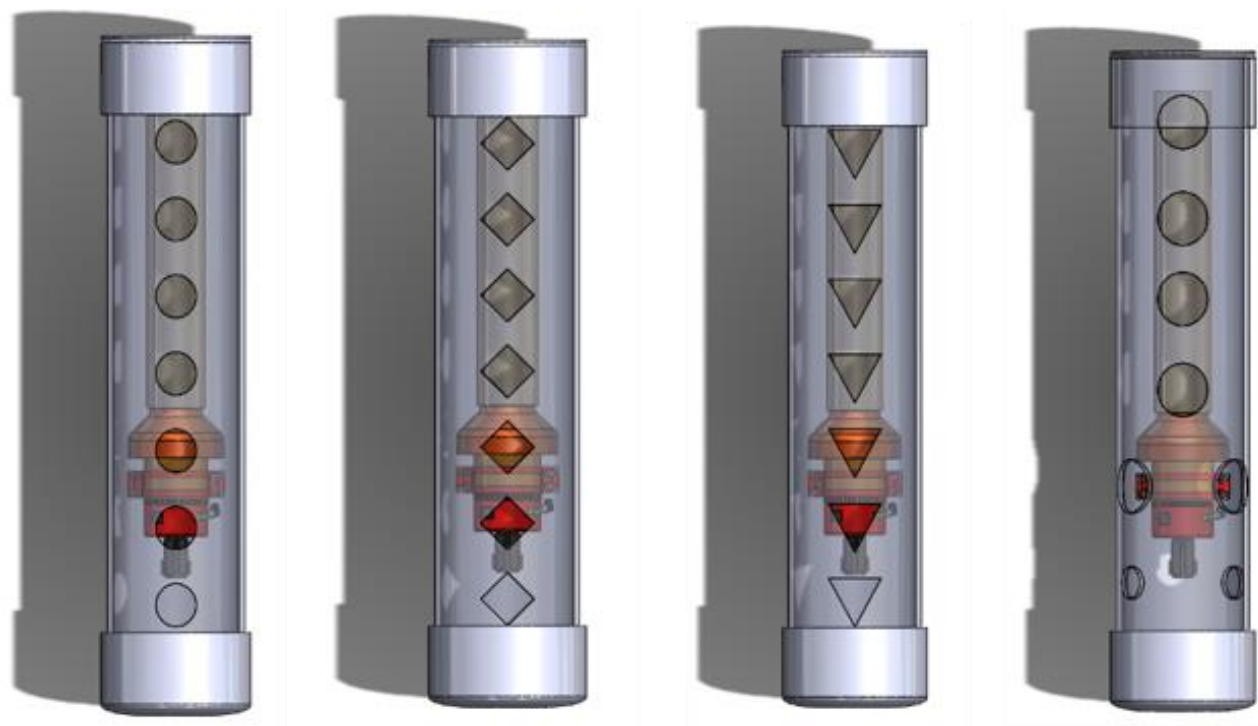

Figure 74: Test matrix of inlet shape and location used for K45 sizing on prototype 


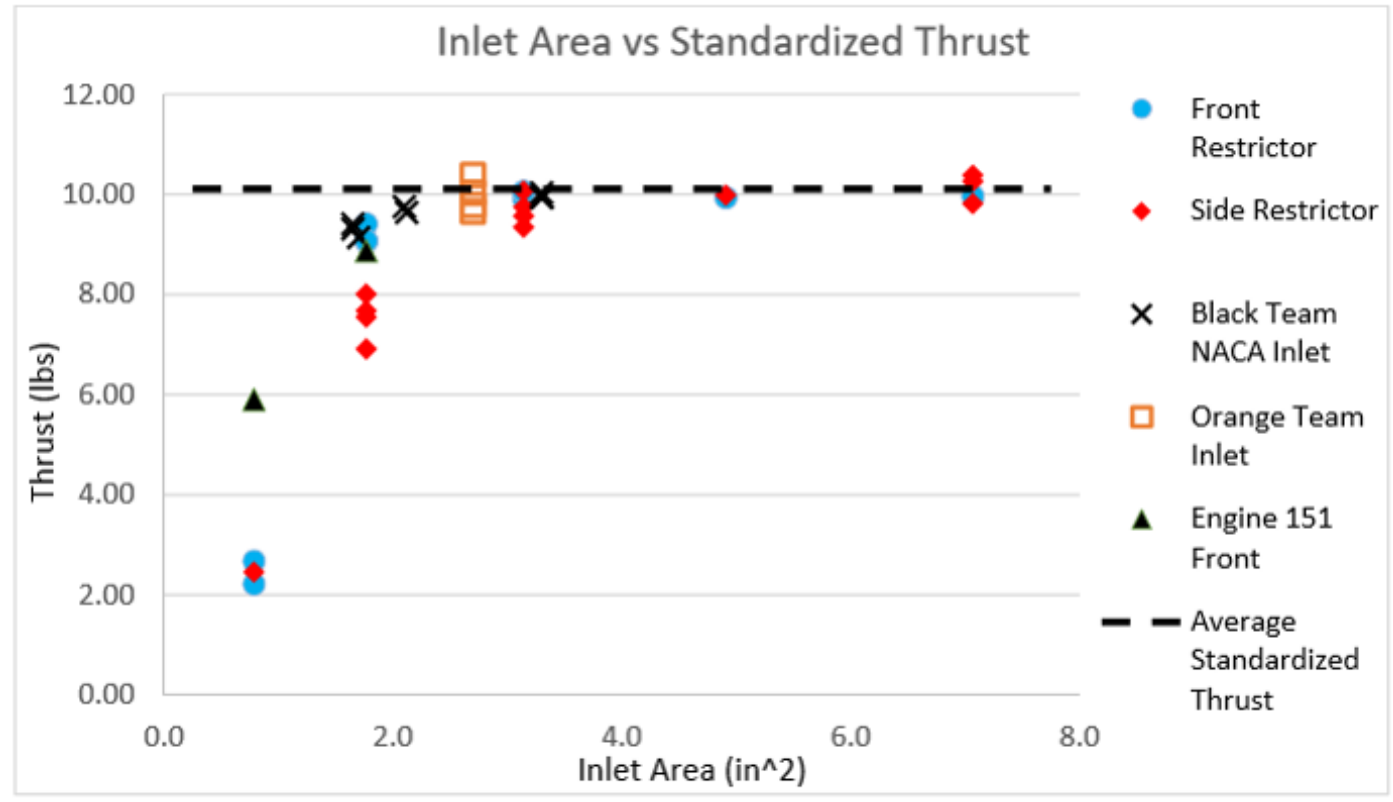

Figure 75: Thrust vs inlet area for Kingtech K45 with no jet pipe ${ }^{24}$. Jet pipe increases required inlet area, but this data was used to guide sizing for $K 70$ inlets

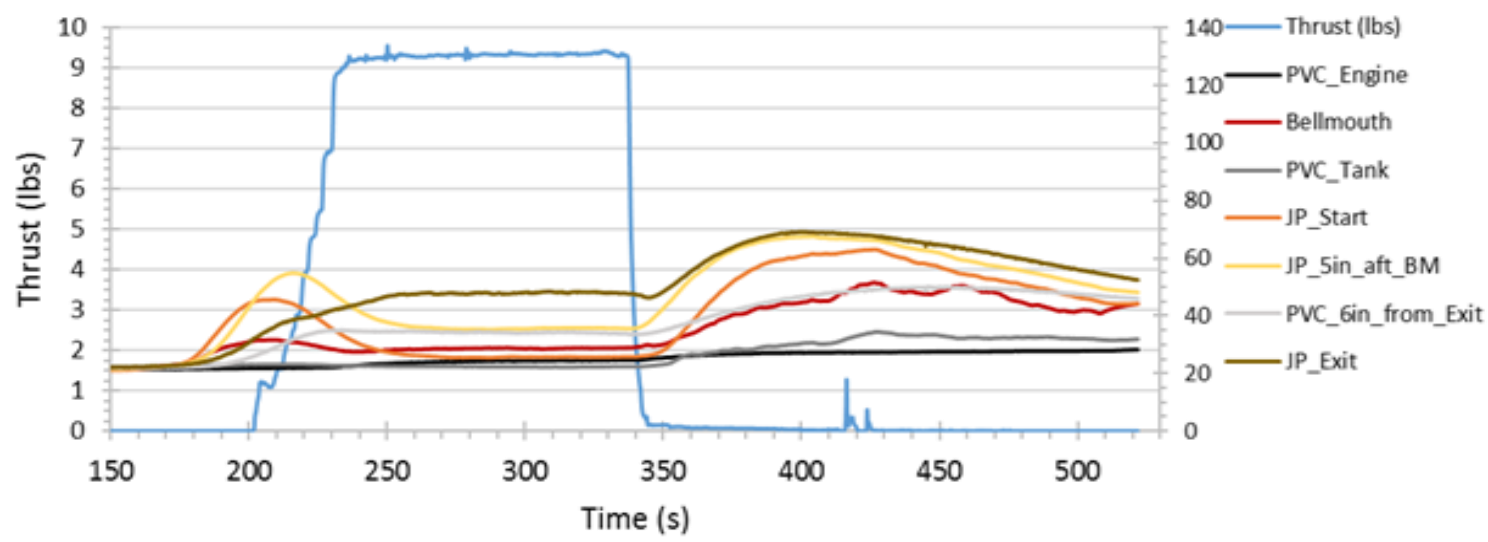

Figure 76: Baseline thrust metrics: open chamber with no flow restriction 


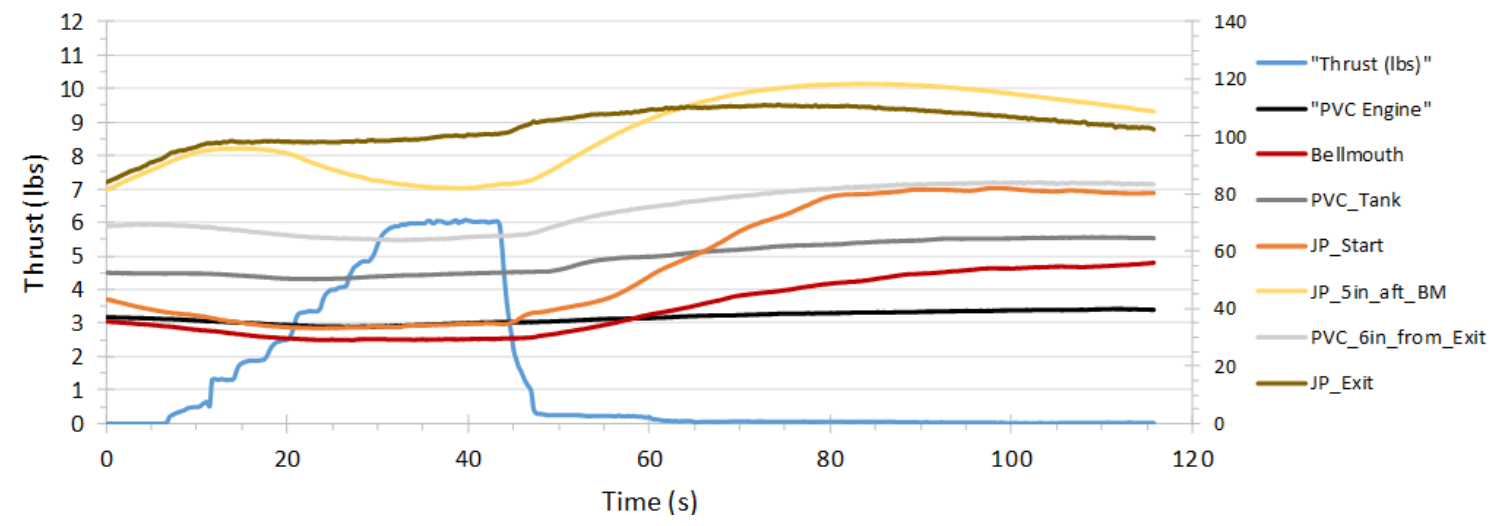

Figure 77: Single inlet (6.3 $\mathrm{in}^{2}$ inlet area) above turbine. Turbine is choked

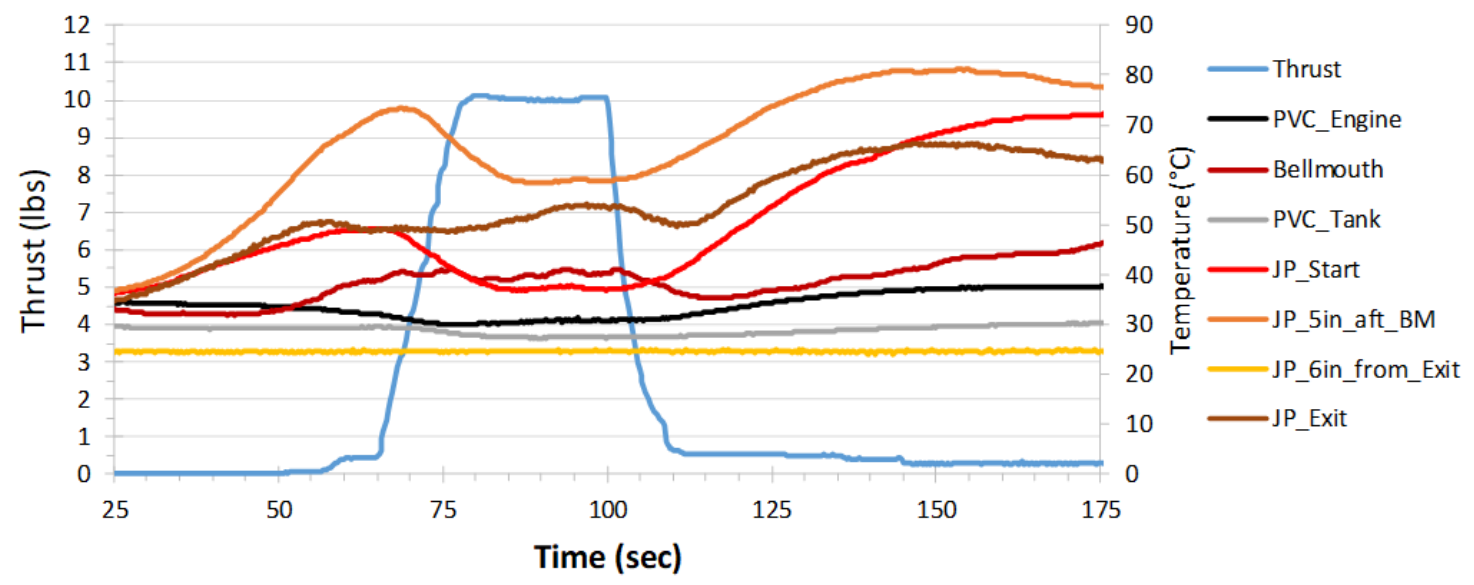

Figure 78: Dual inlets (9.8 $\mathrm{in}^{2}$ total inlet area) above turbine. Better performance, slightly exceeds baseline. Jet pipe increases thrust performance.

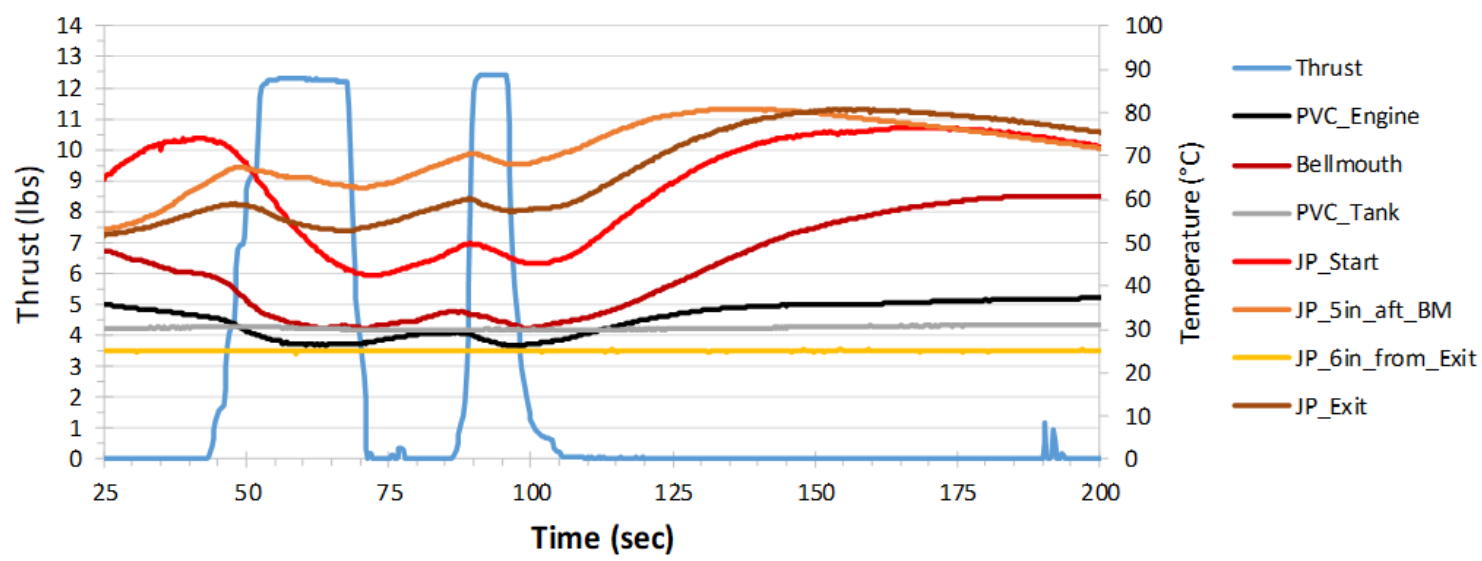

Figure 79: Single inlet (12 in ${ }^{2}$ inlet area) above turbine. Best performing inlet choice, was used for prototype. 


\begin{tabular}{|c|c|c|c|c|c|c|c|c|c|}
\hline $\begin{array}{c}\text { ThrottlePosition } \\
{[\%]}\end{array}$ & RPM & $\begin{array}{l}\text { Estimated } \\
\text { Thrust [lb] }\end{array}$ & $\begin{array}{l}\text { Initial Fuel } \\
\text { Weight [oz] }\end{array}$ & $\begin{array}{c}\text { Final Fuel } \\
\text { Weight [oz] }\end{array}$ & $\begin{array}{l}\text { Fuel Burn } \\
\text { Time [sec] }\end{array}$ & $\begin{array}{c}\text { Exhaust Gas } \\
\text { Temperature } \\
{[\mathrm{C}]}\end{array}$ & $\begin{array}{l}\text { Load Cell Volt } \\
\text { [V] }\end{array}$ & Thrust [lb] & $\begin{array}{l}\text { Fuel Burn Rate } \\
\text { [oz/min] }\end{array}$ \\
\hline 0 & 54900 & 0 & 93.25 & 92.95 & 13.36 & 476 & $3.23 \mathrm{E}-05$ & 0.00 & 1.35 \\
\hline 10 & 82500 & 1 & 90.5 & 90.15 & 10.43 & 452 & 3.27E-04 & 1.02 & 2.01 \\
\hline 20 & 96600 & 2 & 88.4 & 87.7 & 15.26 & 448 & $5.84 \mathrm{E}-04$ & 1.91 & 2.75 \\
\hline 30 & 109900 & 3 & 79.7 & 79.05 & 15.2 & 448 & 8.55E-04 & 2.85 & 2.57 \\
\hline 40 & 121600 & 4 & 75.8 & 75.15 & 15.19 & 452 & $1.16 \mathrm{E}-03$ & 3.93 & 2.57 \\
\hline 50 & 130400 & 5 & 92.05 & 90.95 & 15.29 & 488 & $1.47 \mathrm{E}-03$ & 5.00 & 4.32 \\
\hline 60 & 141500 & 7 & 88.4 & 87.25 & 15.39 & 496 & $1.85 \mathrm{E}-03$ & 6.31 & 4.48 \\
\hline 70 & 151500 & 8 & 82.2 & 80.75 & 15.4 & 524 & $2.32 \mathrm{E}-03$ & 7.92 & 5.65 \\
\hline 80 & 161200 & 9 & 76.8 & 75.2 & 15.33 & 544 & 2.79E-03 & 9.57 & 6.26 \\
\hline 89 & 169500 & 11 & 61.8 & 60.15 & 15.37 & 568 & $3.25 \mathrm{E}-03$ & 11.17 & 6.44 \\
\hline 100 & 180500 & 13 & 82.05 & 80.2 & 15.18 & 592 & $3.89 E-03$ & 13.37 & 7.31 \\
\hline
\end{tabular}

Table 10: Thrust cell data of Kingtech K70G2 turbine used in TIA

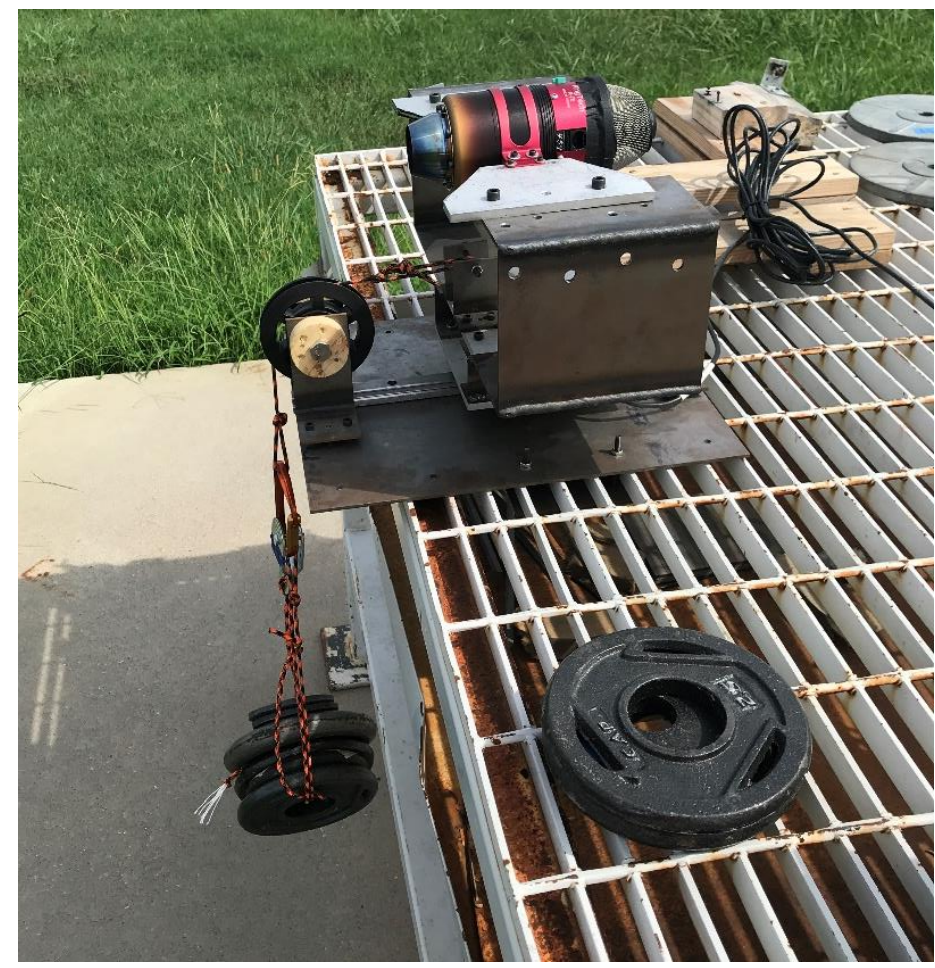

Figure 80: K70G2 turbine used on TIA mounted to thrust cell undergoing calibration

An interesting observation that was not acted upon but could be warrant further research: reverse flow. 


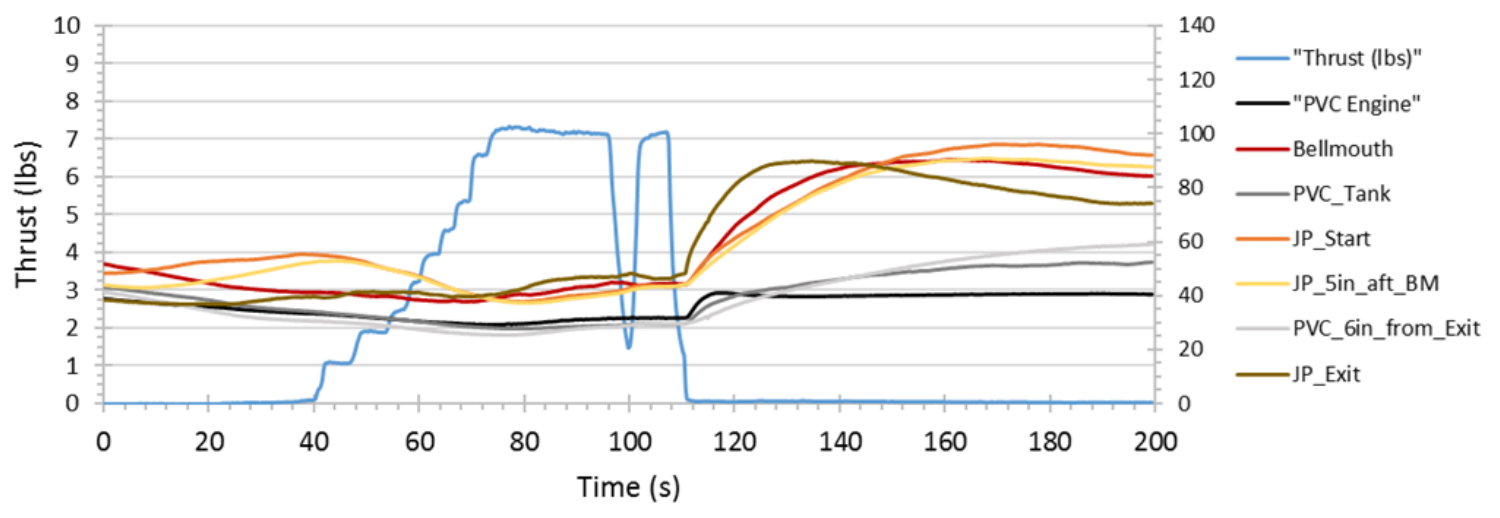

Figure 81: Rear inlet area: 9.7in ${ }^{2}$ open area around jet pipe. Blocked front inlets, pure reverse flow in 3ft long 6" diameter PVC pipe.

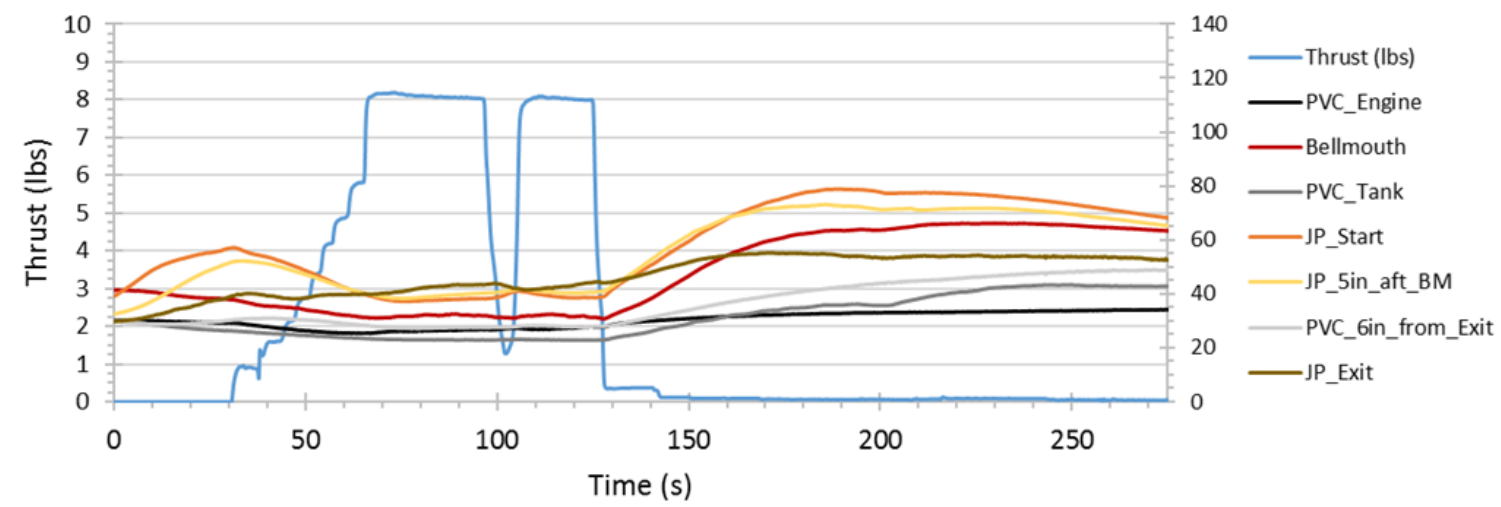

Figure 82: Rear inlet area: $25.4 \mathrm{in}^{2}$ open area around jet pipe. Blocked front inlets, pure reverse flow in $3 f$ tong 6" diameter PVC pipe.

Though these plots look promising from a thrust standpoint, these are done in a static environment, which would have reduced performance when in flight. That said, the turbine is capable of operating entirely off of reverse-flow conditions provided the proper rear intake area is available, as that required intake increases by a minimum factor of 2 , true limit for similar optimal performance was not found. 


\section{APPENDIX C: STRUCTURES DATA}

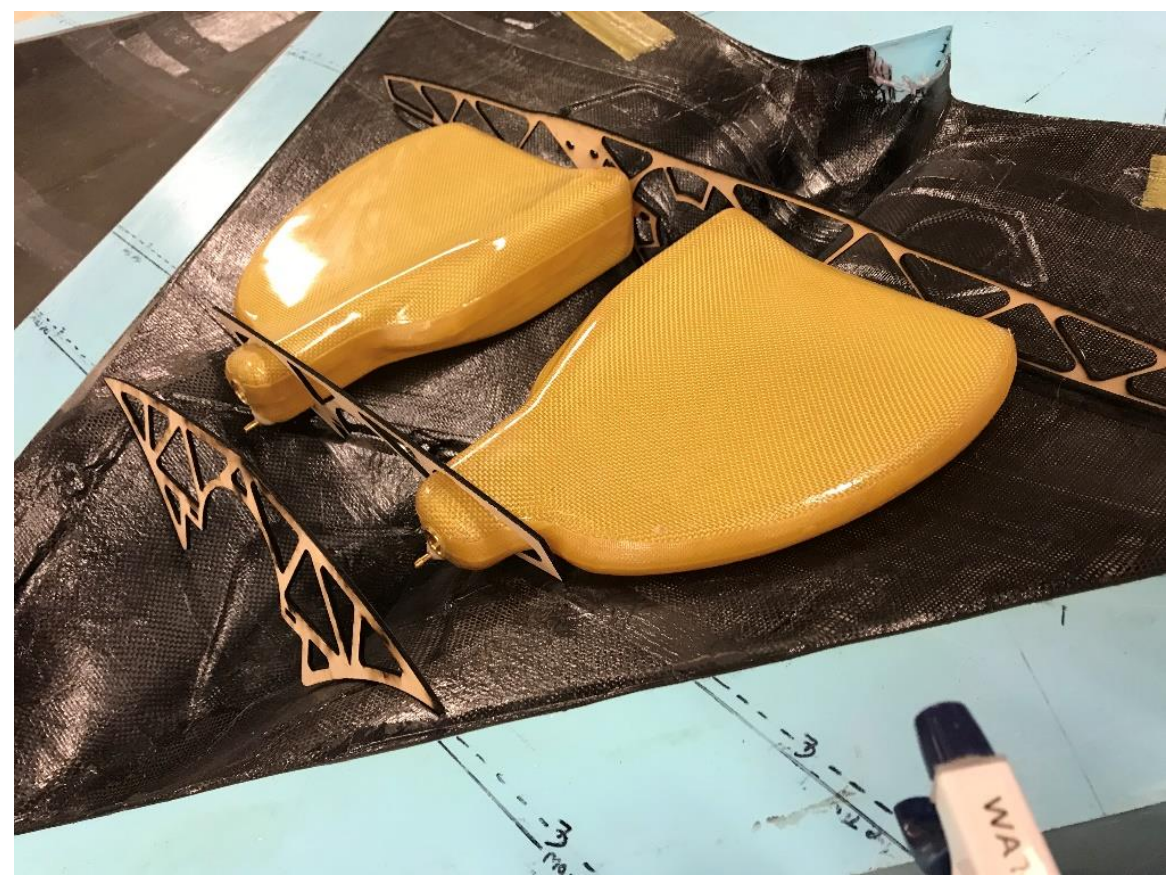

Figure 83: Fuel tanks are non-removable, they are installed during airframe manufacturing 


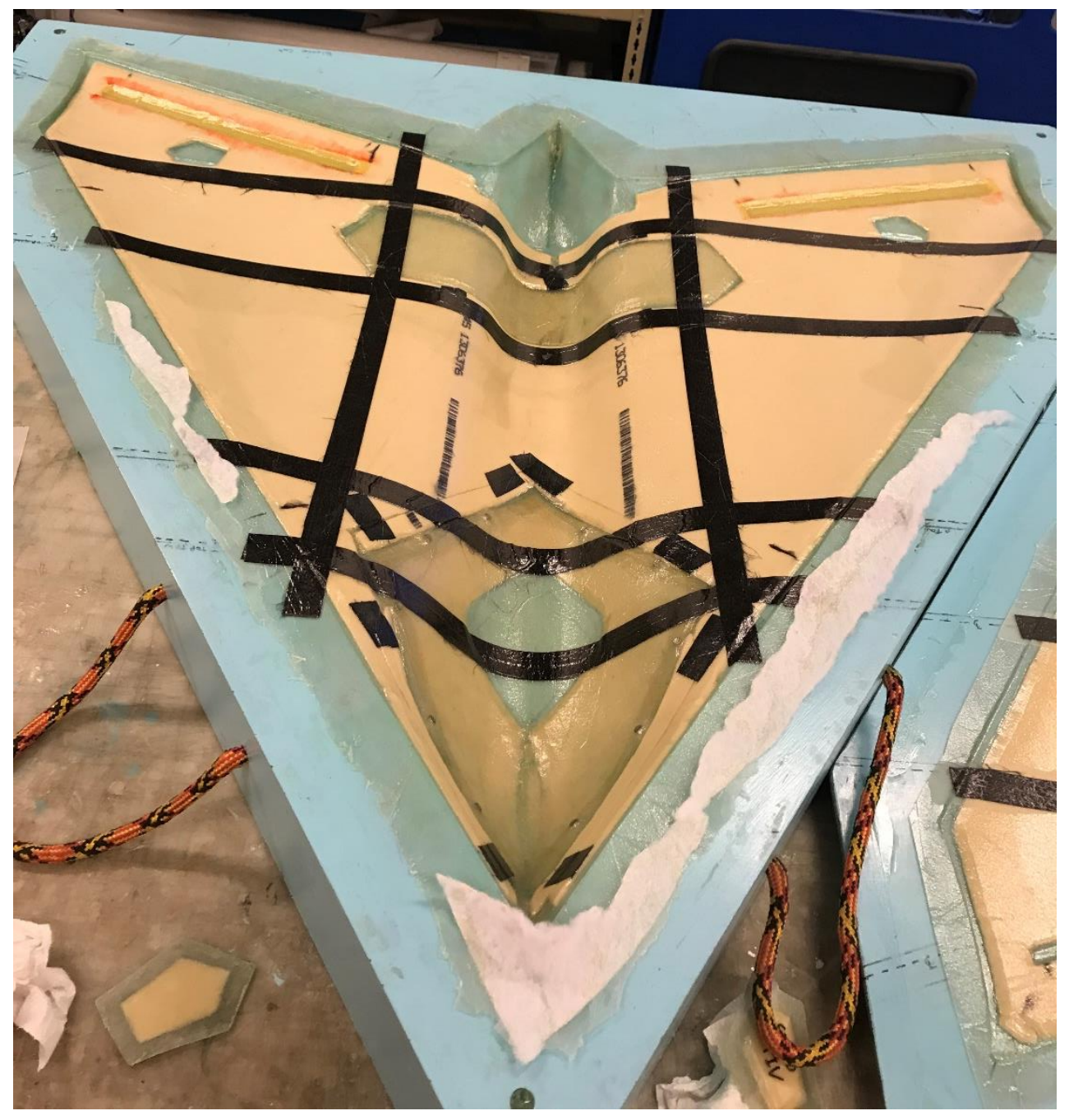

Figure 84: Top skin after layup but before removal. Hatches can be seen under skin 


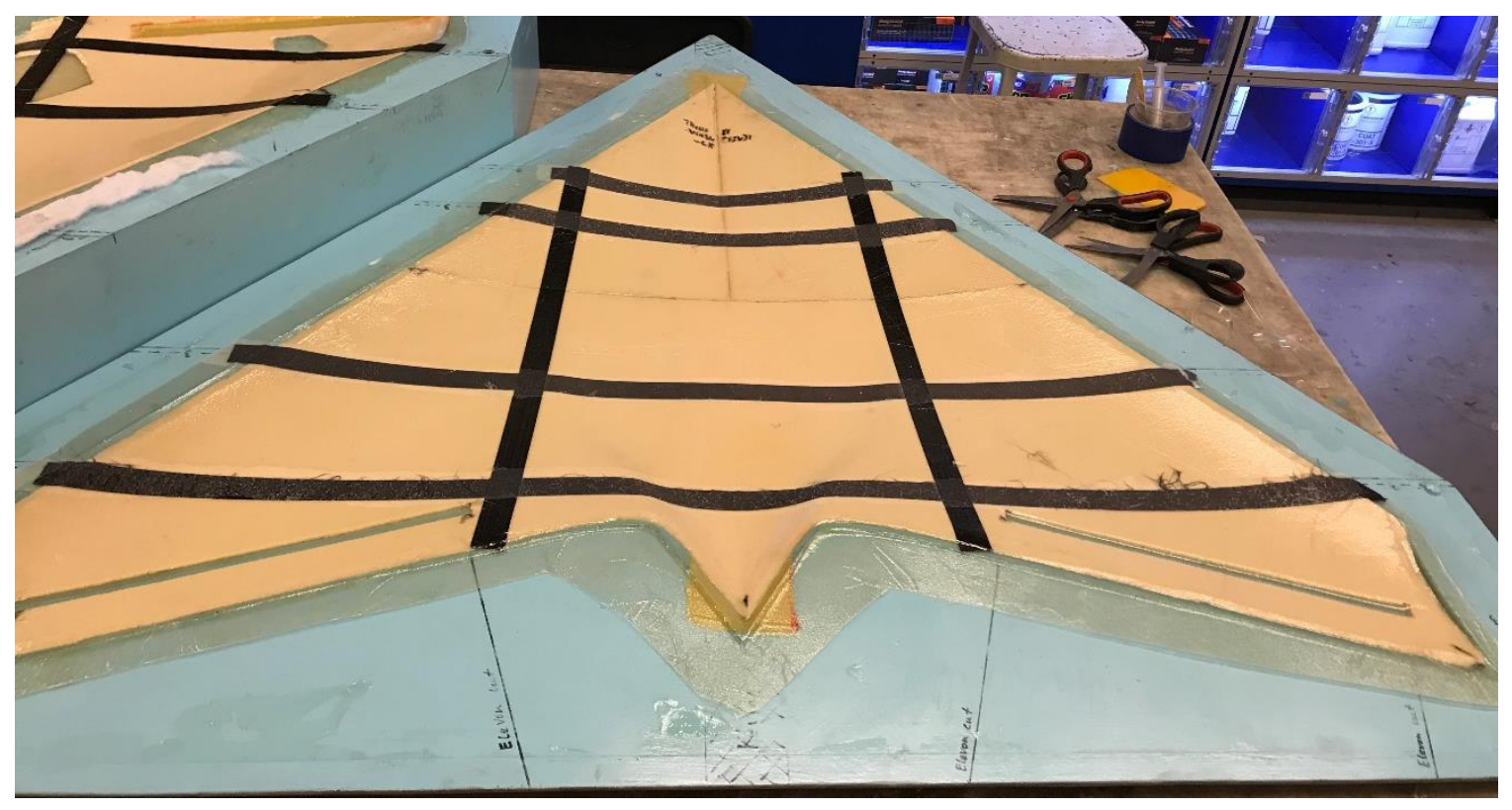

Figure 85: Bottom skin after layup but before removal

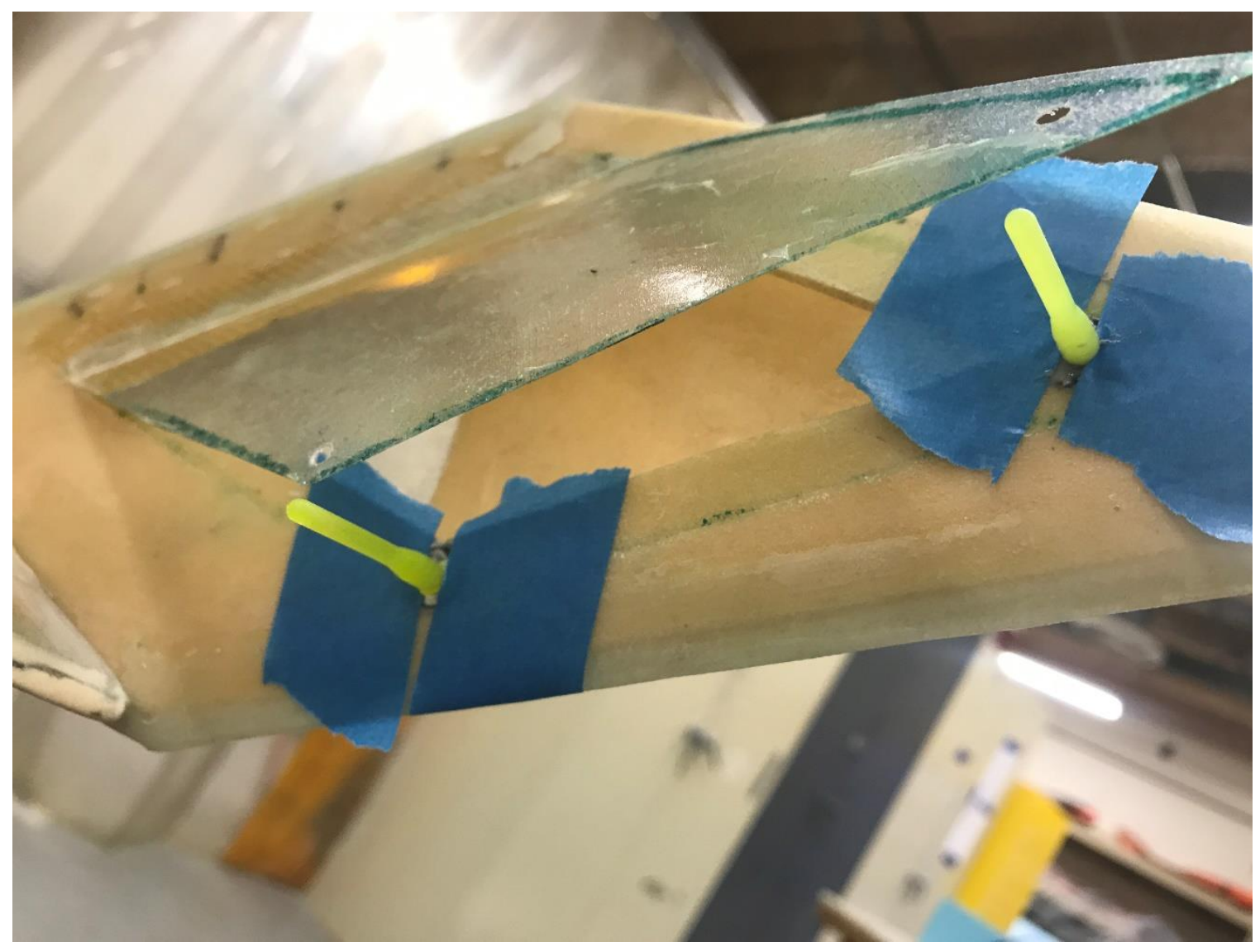

Figure 86: Installing threaded inserts to winglet hatch 


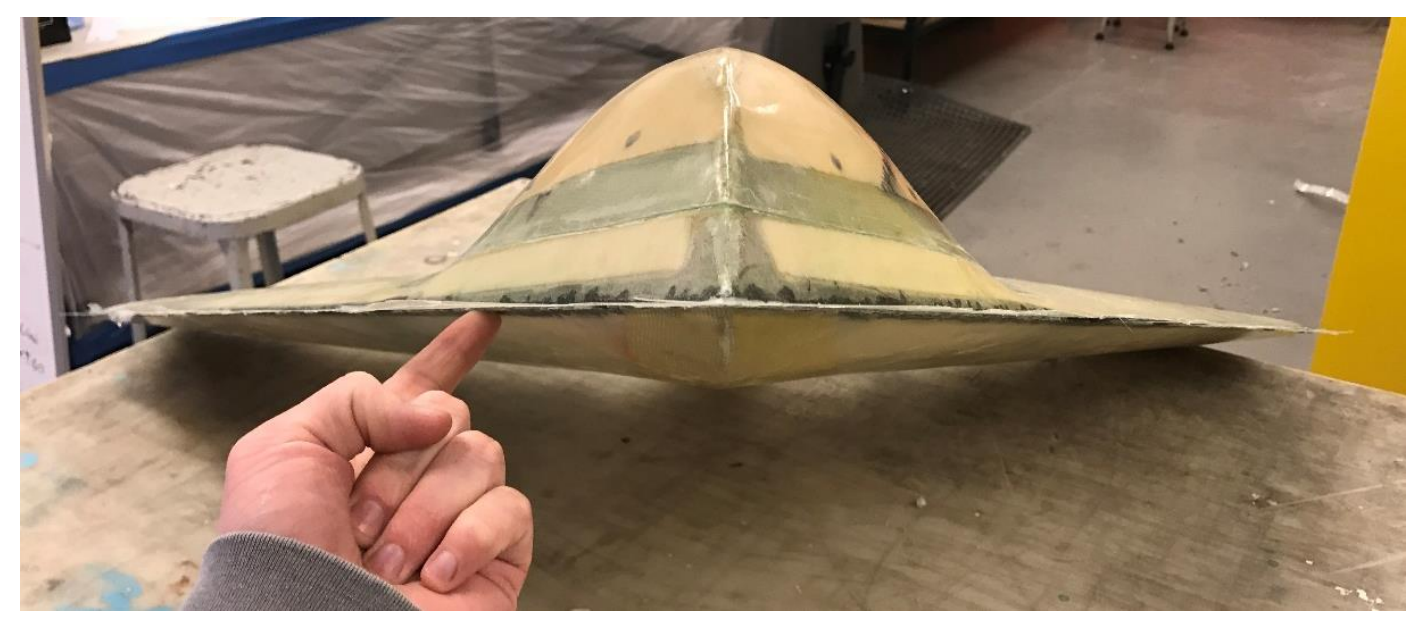

Figure 87: Airframe after bonding halves together (winglets uninstalled)

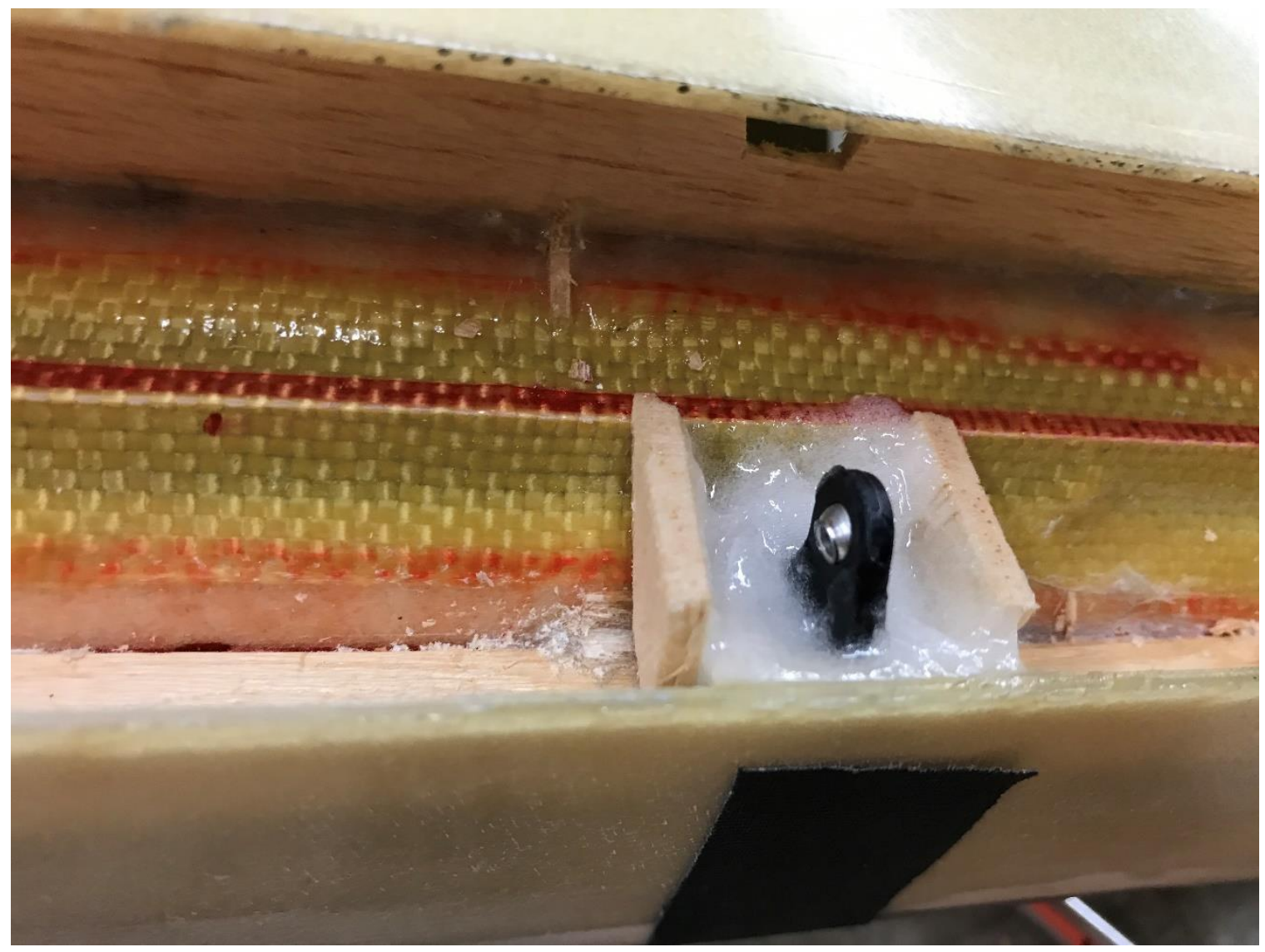

Figure 88: Elevon hinge line showing holes for servo linkage pass-through 


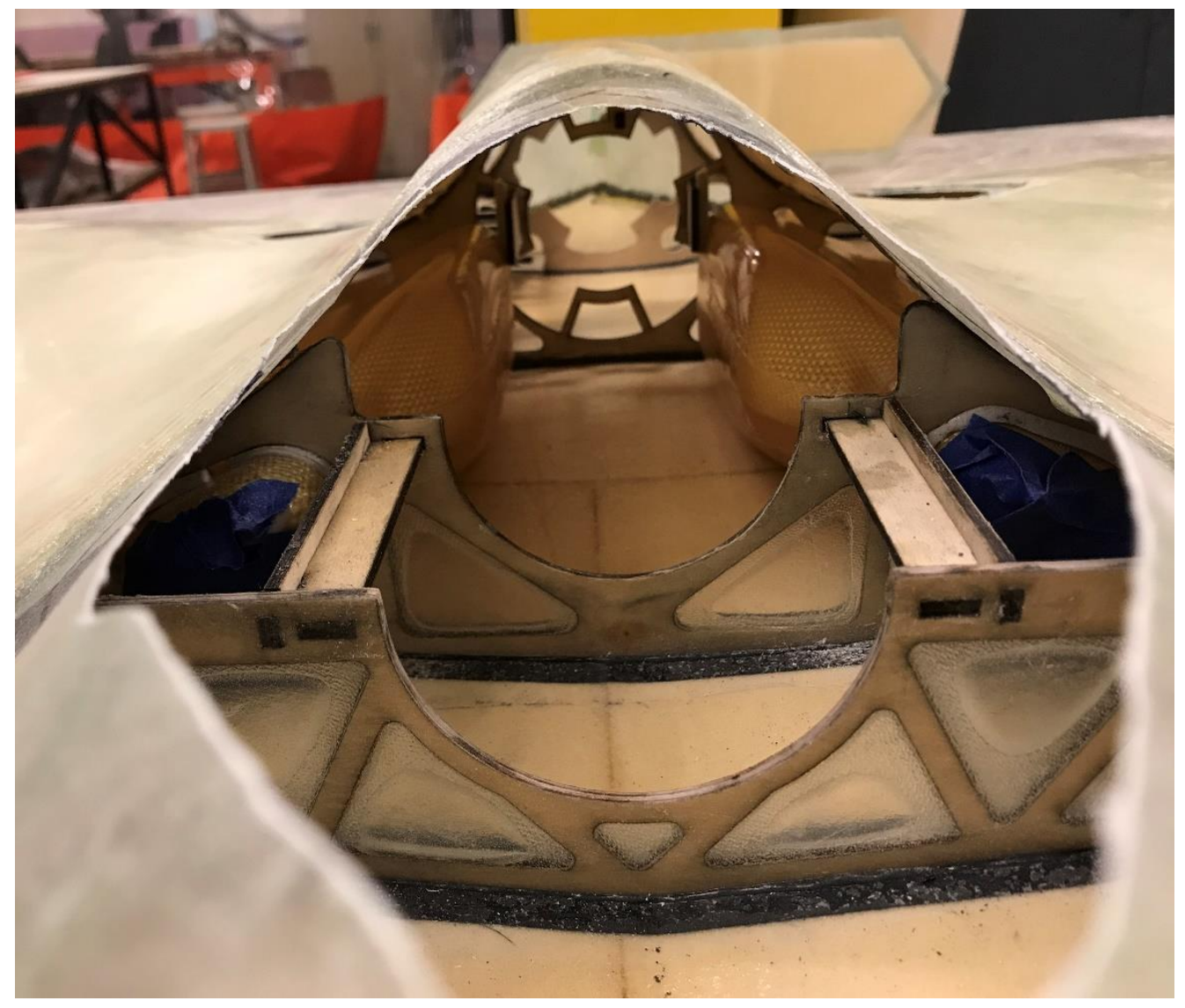

Figure 89: Aft view from the nose of the aircraft prior to component installation 


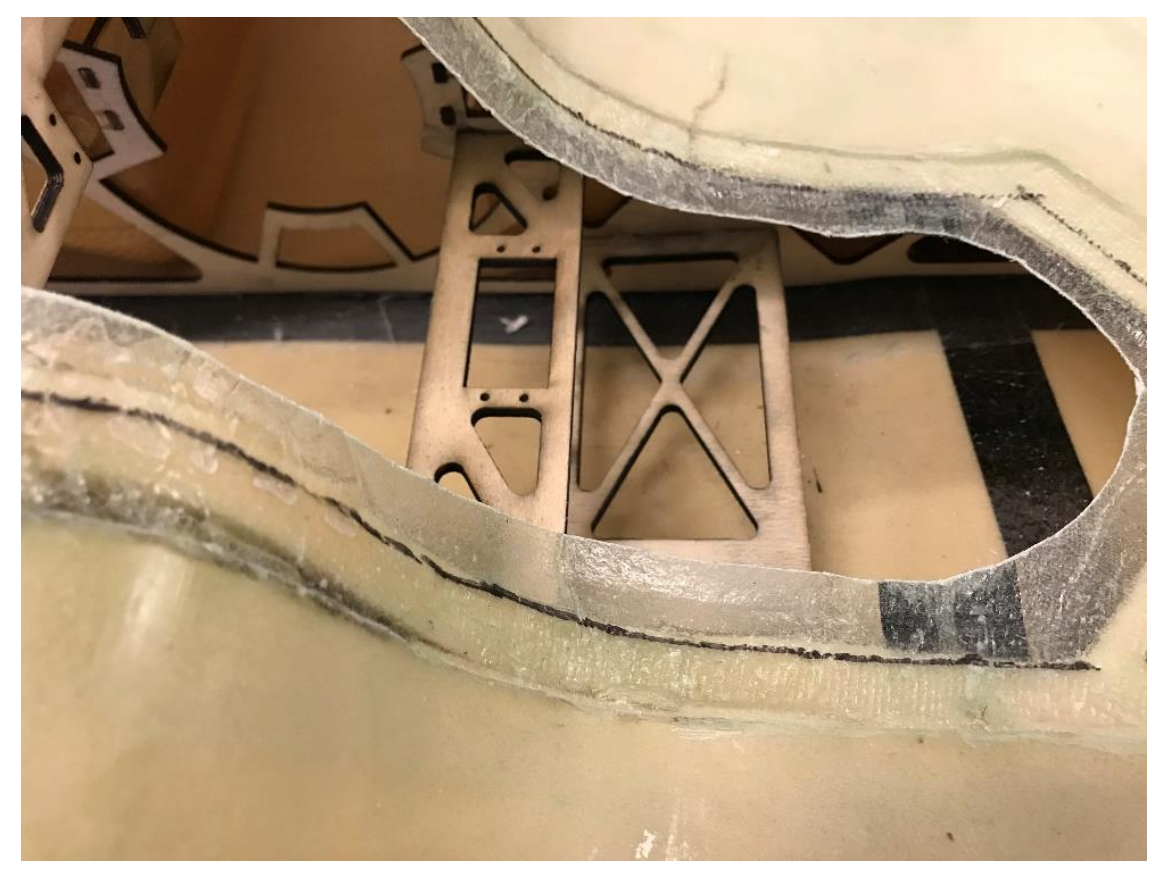

Figure 90: Rear starboard avionics bay prior to component installation.

\begin{tabular}{|c|c|c|}
\hline$C_{n \beta}$ & \multicolumn{2}{|c|}{ Area } \\
\hline 0.05 & 42 & $i^{2}$ \\
\hline 0.1 & 68 & $i^{2}$ \\
\hline 0.2 & 116 & $\mathrm{in}^{2}$ \\
\hline
\end{tabular}
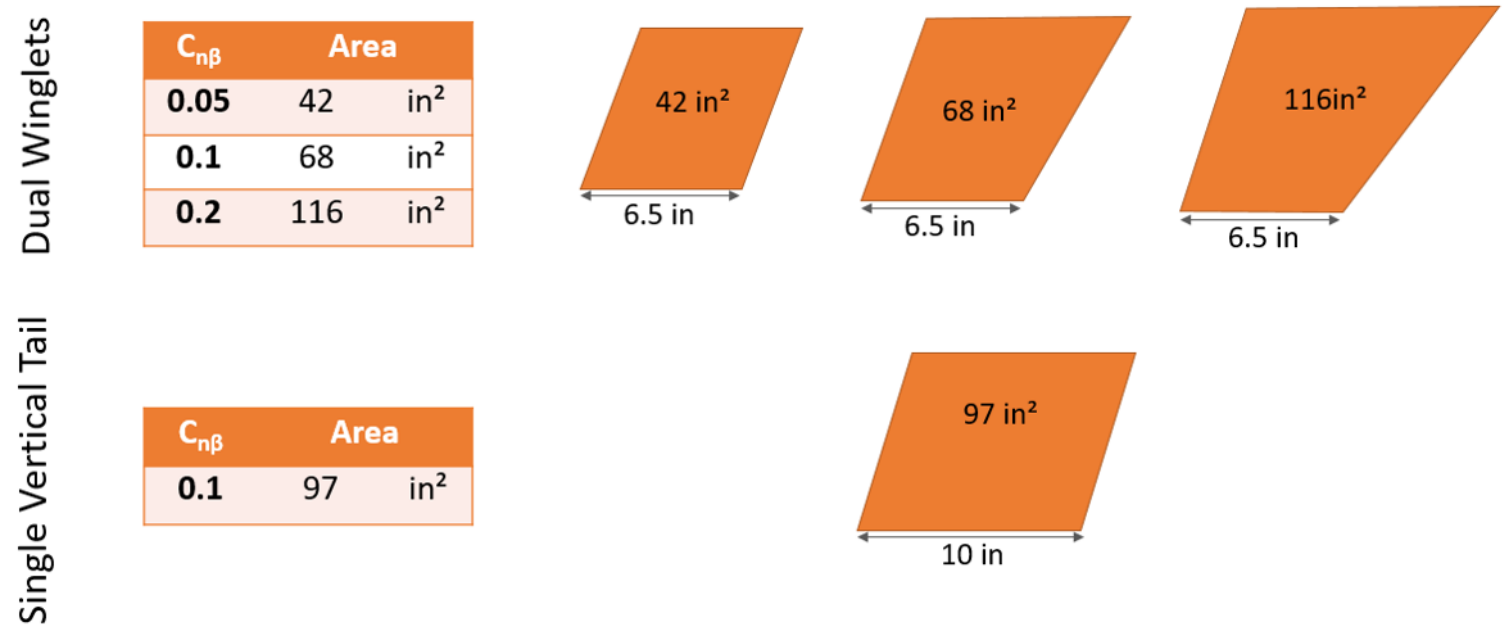

Figure 91: Test matrix of winglets from Speedfest. The $68 \mathrm{in}^{2}$ winglets had optimal performance for its size, with winglets outperforming a single vertical tail in high AoA landings. 
APPENDIX D: LAUNCHER WIND LOAD ESTIMATE

\begin{tabular}{|c|c|}
\hline \begin{tabular}{c} 
Ground Speed $(\mathrm{mph})$ \\
\hline $\begin{array}{c}\text { Air Density } \\
\left(\text { slug } \mathrm{ft}^{\wedge} 3\right)\end{array}$
\end{tabular} & 0.0023769 \\
\hline$\left(\mathrm{N}^{\wedge} 2\right) / 2\left(\mathrm{ft}^{\wedge} 2 / \mathrm{s}^{\wedge} 2\right)$ & 7771 \\
\hline $\begin{array}{c}\text { Aircraft Planform } \\
\text { Area (in^ } 2)\end{array}$ & 0 \\
\hline C_D of Aircraft & 0.068 \\
\hline $\begin{array}{c}\text { Frontal Area of } \\
\text { Launcher (in^ } 2)\end{array}$ & 90 \\
\hline C_D of launcher & 2.1 \\
\hline Est. Wind Force (lbs) & 24 \\
\hline Factor of Safety & 4 \\
\hline Max Wind Load (lb) & 97 \\
\hline
\end{tabular}

Table 11: Wind loading estimations for the launcher's structure 


\section{APPENDIX E: ATTEMPTED AIRFOILS FOR XFOIL ANALYSIS}

XFOIL does not work well with sharp leading edge airfoils (as biconvex airfoils are), which meant the airfoil file would need to be modified at a very small scale to give XFOIL the required curvature. This attempt was done in XFOIL itself using the airfoil modification command set. Using this modified airfoil, XFOIL results were able to be captured, but not fully. As the program cycled through angles of attack $\left(-4^{\circ}\right.$ to $10^{\circ}$ in $0.5^{\circ}$ increments), many points failed, with only a few finite angles returning proper values. This led to the use of a less representative but still functionally similar airfoil, the NACA 0010-35.

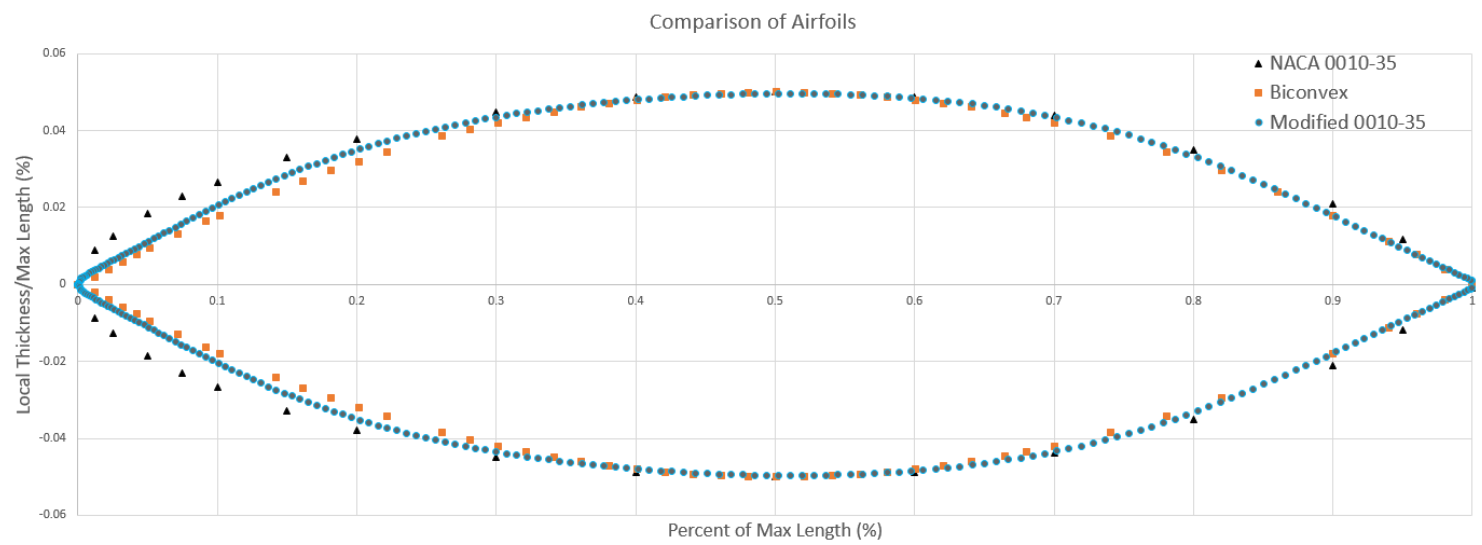

Figure 92: Graphical comparison of Biconvex, NACA 0010-35, and a Modified NACA 0010-35 airfoils

The NACA 0010-35 is not identical to the Biconvex, so it needed to be tweaked, primarily on the leading edge as the trailing half of all the airfoils are nearly identical. To do so, the same process in XFOIL that was originally intended to tweak the Biconvex airfoil was used on the 0010-35. In particular, the leading edge curvature was reduced dramatically. There are two settings that control this in XFOIL: the radius and degree of curvature. 
Since there were not analytical methods of comparing the modified airfoil to the Biconvex, a graphical approach was used. This involved taking the text files that were output from XFOIL and plotting them in Excel, which resulted in Figure 92. Once the modified airfoil reached a sufficient level of similarity, it was then run in XFOIL. The same issues arose from this as did the previous attempts of a pure biconvex airfoil: intermittent failures of various angles of attack. Backtracking yet further, the base version of the modified airfoil, the NACA 0010-35, was used.

The base version of the NACA 0010-35 was the first of the non-standard airfoils tested that yielded functional results. Though this deviates from the true Biconvex airfoil significantly along the leading edge, there is less deviation from what exists on the actual aircraft. Due to the manufacturing process, there was a slight ogive present on the front of the leading edge, which actually gives the leading edge a (relatively) tall, rounded leading edge rather than a pointed leading edge. With this consideration and the overall slight difference between Biconvex and NACA 0010-35 as seen in Error! Reference source not found., the 0010-35 was used for a nalysis. Note: even if the actual Biconvex airfoil was used, this process only yields an estimate with a large margin of error due to the geometry of the airframe. As such, this deviation in airfoil type will not yield such a different result that the same analysis could not be conducted, so this airfoil was used.

The advantage of using the NACA 0010-35 was actual results could be gathered across a full range of angles of attack $\left(-4^{\circ}\right.$ to $\left.10^{\circ}\right)$, which gave 2-D lift and drag coefficients. Both Biconvex and the modified NACA $0010-35$ were able to give results between $-5^{\circ}$ and $5^{\circ}$, so for those data sets were calculated as well for reference. These data points were used to help feed a basic analytical model that was scrapped due to high level of inaccuracies. 


\section{APPENDIX F: FOAMBOARD FLIGHT TESTING}

Initial planform analysis was conducted using FlightTest foam board panels. These panels were cut to specific sizes and shapes to be formed into various structures, resulting in both a universal fuselage bay as well as various types of wings. The fuselage box was a single sheet scored on one side then folded over on itself with a thin layer of plywood in the center, using hot glue to bond the foamboard to the wood on both sides. Velcro was applied down the center to hold the batteries down as well as battery straps across them. The EDF was mounted in its own foamboard shroud and hot glued to the main fuselage structure. This can be seen below:

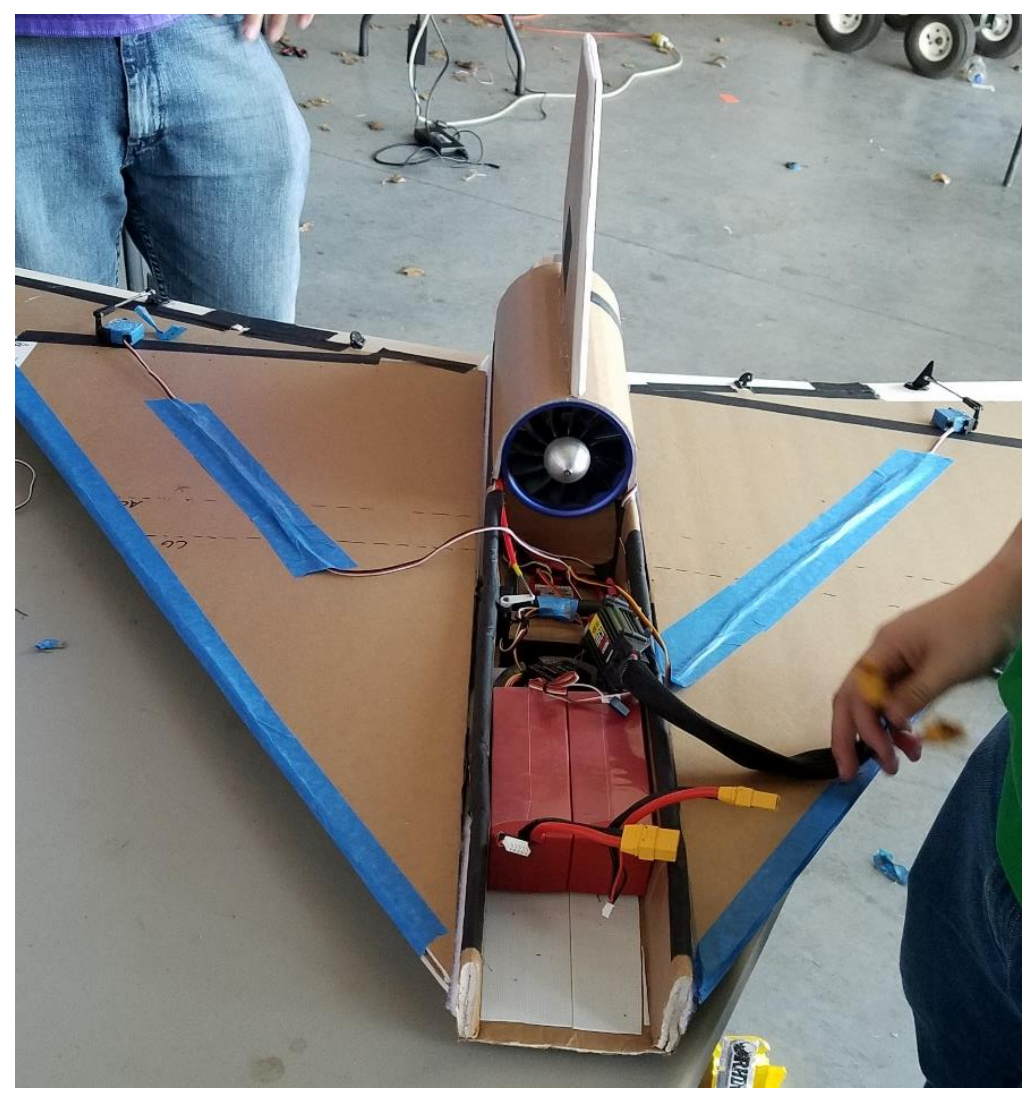

Figure 93: Front view of foamboard prototype 
The wings were created in much the same way with the exception of no plywood structure. The internal structure of the wings was comprised of FlightTest foamboard ribbing throughout with a cardboard cylinder end cap secured inside the wing. A carbon tube was run through the fuselage with an end in each wing for structural support during flight. Since the fuselage and wings were to be modular, both faces of the wing-fuselage interface was covered in Velcro to allow for easy removal. The below figure shows one of the wings that was used. Prototype wings (such as the one shown) had control surfaces attached full-length off the trailing edge, whereas refined products nearing the final integration step had integrated control surfaces.

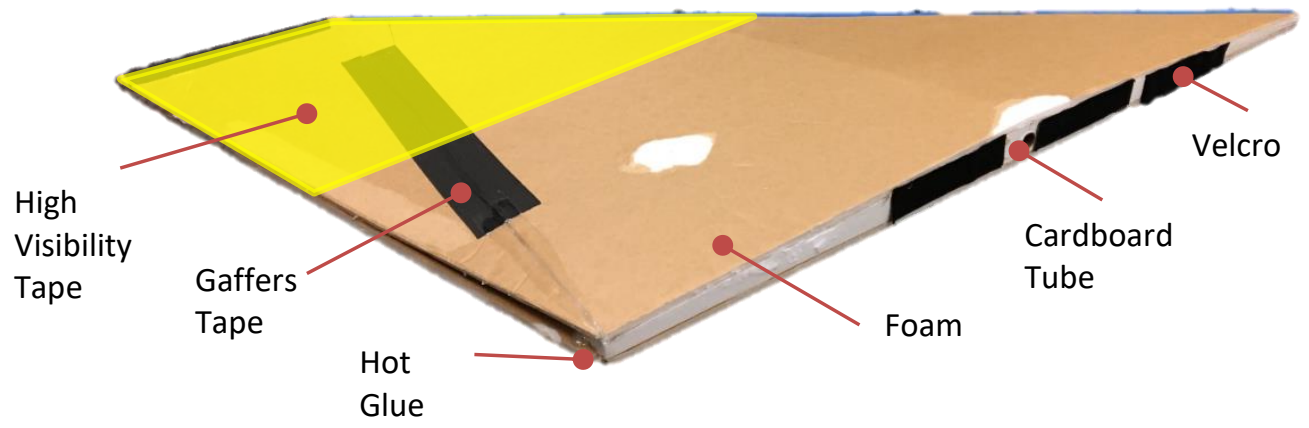

Figure 94: Foamboard wing structure

These processes were used to create the initial foamboard aircraft that allowed for targeted testing of high angle of attack flight maneuvers. Once a planform was finalized and chosen, an intricate foamboard model that closely mirrored the final composite airframe design was created. This foamboard aircraft had very similar fuselage and wing thickness dimensions as well as a similar losses due to a blunt-nosed aircraft. This aircraft can be seen below: 


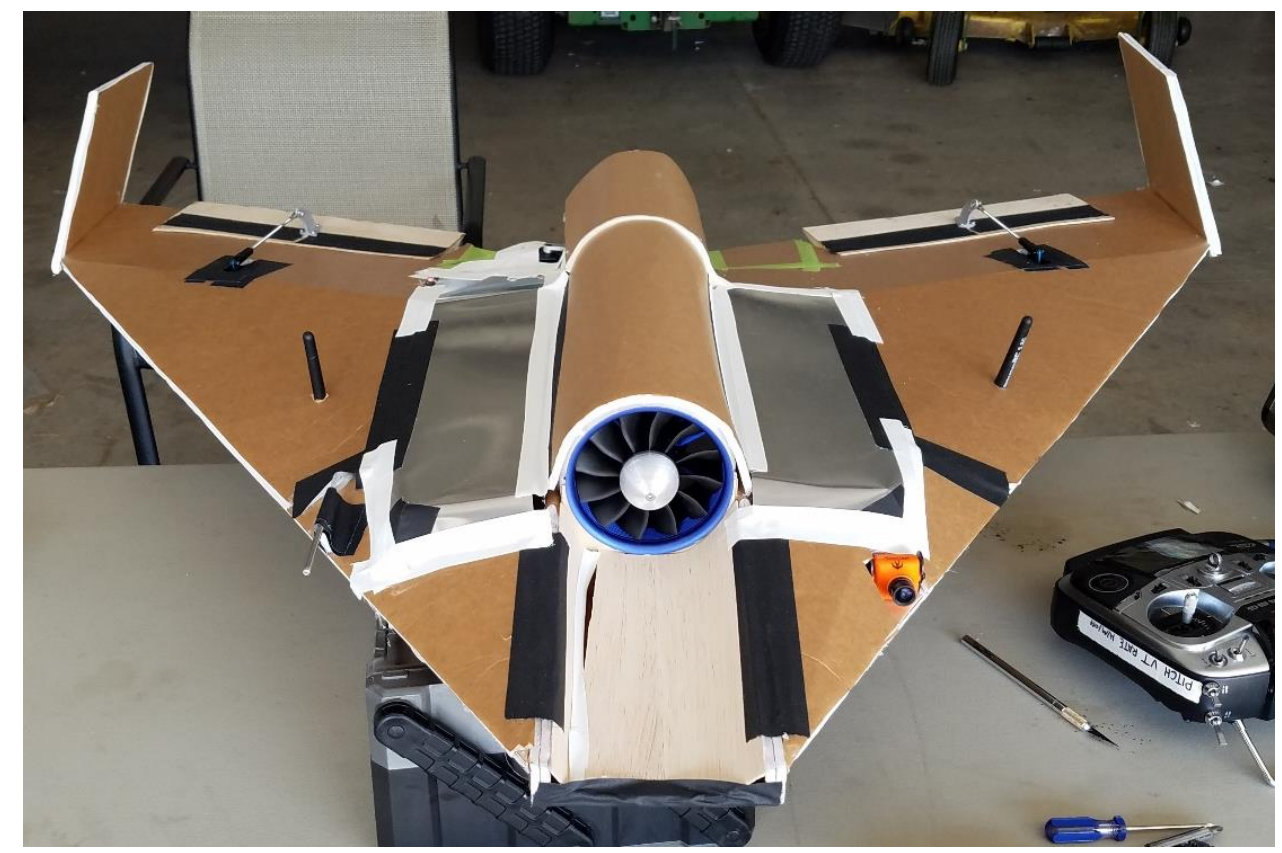

Figure 95: Final foamboard model that simulates full composite airframe

The purpose of this final version was for initial tuning of the Aura8 rate gyro's initial PID gains. This ensured the prototype composite aircraft would have as much chance for survival during its maiden flight as possible. This held to be true, as both the foamboard and composite airframes were flown successfully with no issues arising from the rate gyros. 


\section{APPENDIX G: MATLAB FLIGHT DATA PLOTTING CODE}

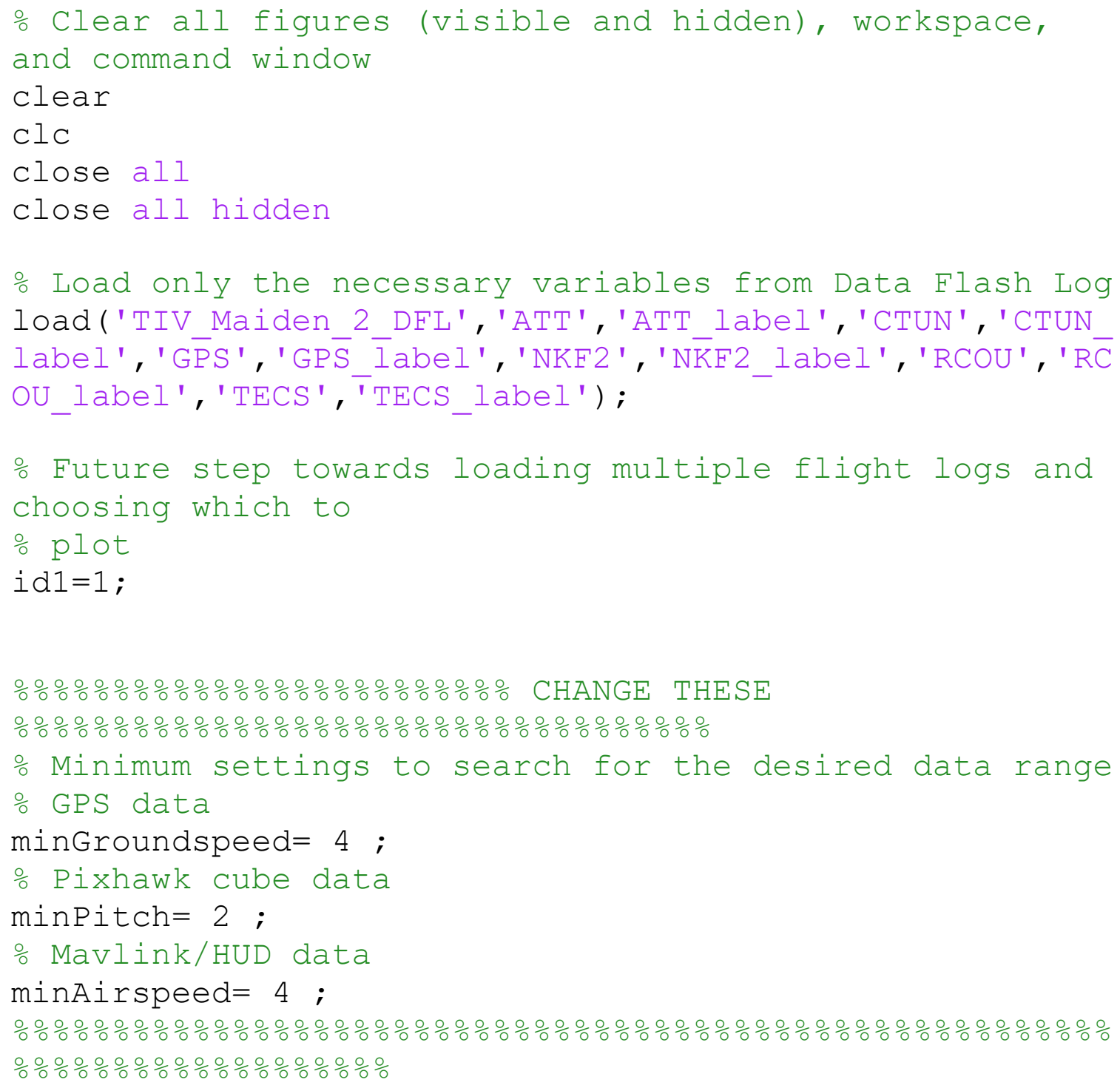




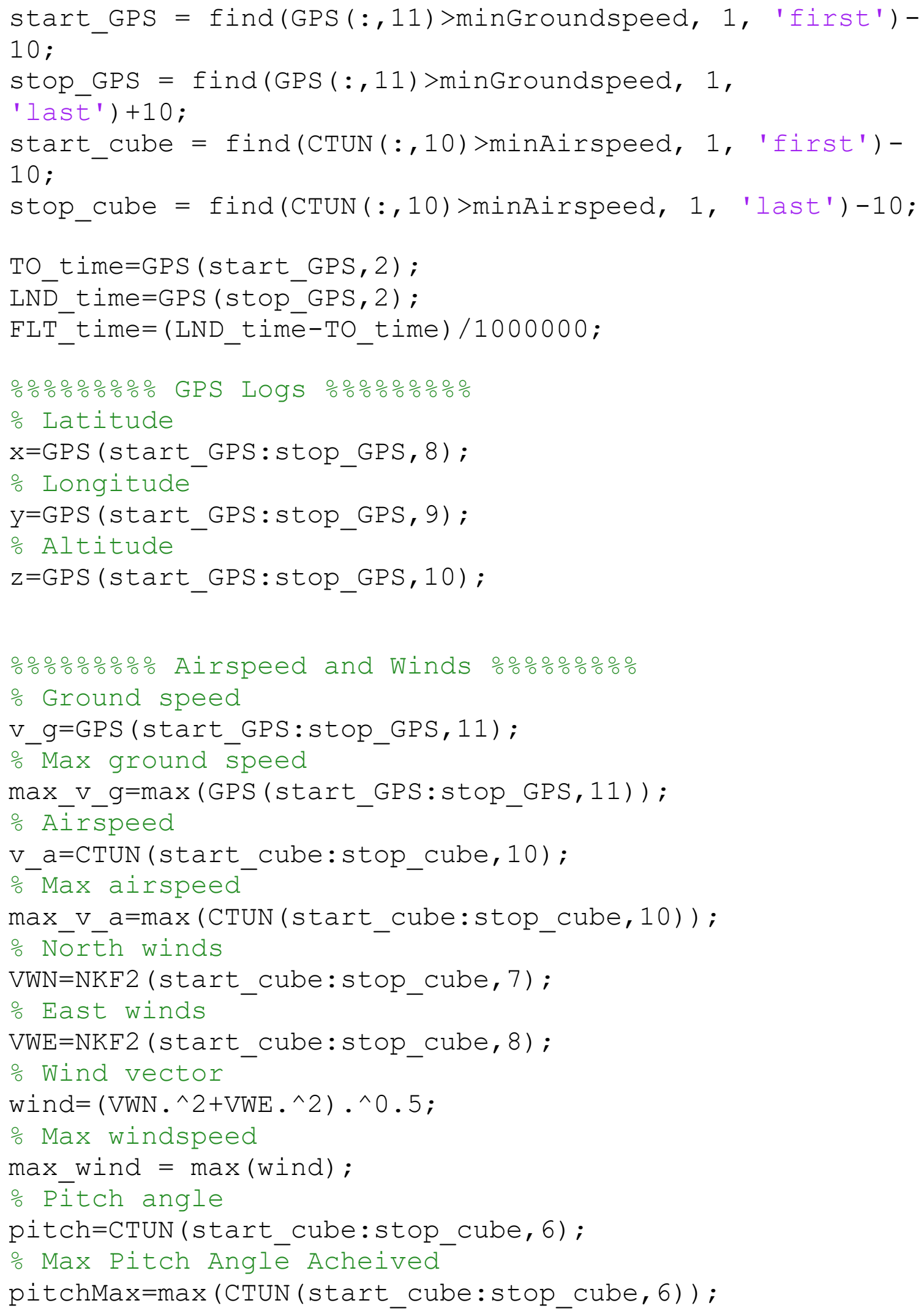


\% Pixhawk time stamps converted into seconds

t_GPS = (GPS (start_GPS:stop_GPS, 2) -

GPS (start_GPS, 2))/ $\overline{1} 000000$;

$t$ cube $=($ CTUN $($ start cube $:$ stop cube, 2$)-$

CT̄UN (start_cube, 2))/ 1000000 ;

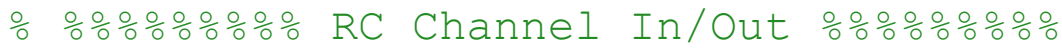

o Throttle output from Pixhawk

thr $=($ RCOU (start_cube:stop_cube, 5)-1100)/(1900-

$1100) \star 100$;

\% Used for dashed line along $x$-axis in plots

zero=zeros (length (pitch)) ;

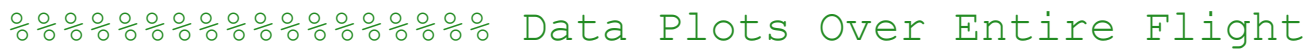

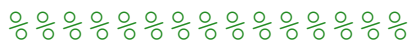

figure (1)

\% Groundspeed plot

subplot $(6,1,1)$

plot ( $t \_G P S, v_{-} g$ )

title ('Groundspeed vs Time')

ylabel ('Groundspeed (m/s) ')

\% Airspeed plot

subplot $(6,1,2)$

plot ( $t$ cube, $v_{-}$a)

title ('Airspeēd vs Time')

ylabel ('Airspeed (m/s)')

\% Windspeed magnitude plot

subplot $(6,1,3)$

plot ( $t$ _cube, wind)

title("Windspeed Magnitude vs Time')

ylabel ('Windspeed (m/s)')

\% Throttle Output

subplot $(6,1,4)$

plot ( $t$ cube, thr)

title('Throttle vs Time')

ylabel ('Throttle (\%)') 


\section{APPENDIX H: FULL PIXHAWK PARAMETER LIST}

$\begin{array}{lrl}\text { ACRO_LOCKING } & 0 & \text { ARSPD_USE } \\ \text { ACRO_PITCH_RATE } & 180 & \text { ARSPD2_TYPE } \\ \text { ACRO_ROLL_RATE } & 180 & \text { AUTO_FBW_STEER } \\ \text { ADSB_ENABLE } & 0 & \text { AUTOTUNE_LEVEL } \\ \text { AFS_ENABLE } & 0 & \text { AVD_ENABLE } \\ \text { AHRS_COMP_BETA } & 0.1 & \text { BATT_MONITOR } \\ \text { AHRS_CUSTOM_PIT } & 0 & \text { BATT2_MONITOR } \\ \text { AHRS_CUSTOM_ROLL } & 0 & \text { BRD_IMU_TARGTEMP } \\ \text { AHRS_CUSTOM_YAW } & 0 & \text { BRD_IO_ENABLE } \\ \text { AHRS_EKF_TYPE } & 2 & \text { BRD_OPTIONS } \\ \text { AHRS_GPS_GAIN } & 1 & \text { BRD_PWM_COUNT } \\ \text { AHRS_GPS_MINSATS } & 6 & \text { BRD_RTC_TYPES } \\ \text { AHRS_GPS_USE } & 1 & \text { BRD_SAFETY_MASK } \\ \text { AHRS_ORIENTATION } & 0 & \text { BRD_SAFETYENABLE } \\ \text { AHRS_RP_P } & 0.2 & \text { BRD_SAFETYOPTION } \\ \text { AHRS_TRIM_X } & -0.05187 & \text { BRD_SBUS_OUT } \\ \text { AHRS_TRIM_Y } & -0.00879 & \text { BRD_SER1_RTSCTS } \\ \text { AHRS_TRIM_Z } & 0 & \text { BRD_SER2_RTSCTS } \\ \text { AHRS_WIND_MAX } & 0 & \text { BRD_SERIAL_NUM } \\ \text { AHRS_YAW_P } & 0.2 & \text { BRD_TYPE } \\ \text { ALT_CTRL_ALG } & 0 & \text { BTN_ENABLE } \\ \text { ALT_HOLD_FBWCM } & 0 & \text { CAM_AUTO_ONLY } \\ \text { ALT_HOLD_RTL } & 10000 & \text { CAM_DURATION } \\ \text { ALT_OFFSET } & 0 & \text { CAM_FEEDBACK_PIN } \\ \text { ARMING_ACCTHRESH } & 0.75 & \text { CAM_FEEDBACK_POL } \\ \text { ARMING_CHECK } & 1 & \text { CAM_MAX_ROLL } \\ \text { ARMING_REQUIRE } & 0 & \text { CAM_MIN_INTERVAL } \\ \text { ARMING_RUDDER } & 0 & \text { CAM_RELAY_ON } \\ \text { ARMING_VOLT_MIN } & 0 & \text { CAM_SERVO_OFF } \\ \text { ARMING_VOLT2_MIN } & 0 & \text { CAM_SERVO_ON } \\ \text { ARSPD_AUTOCAL } & 0 & \text { CAM_TRIGG_DIST } \\ \text { ARSPD_BUS } & 1 & \text { CAM_TRIGG_TYPE } \\ \text { ARSPD_FBW_MAX } & 62 & \text { CAN_D1_PROTOCOL } \\ \text { ARSPD_FBW_MIN } & 25 & \text { CAN_D2_PROTOCOL } \\ \text { ARSPD_OFFSET } & -8.04676 & \text { CAN_P1_DRIVER } \\ \text { AHESE } & & \end{array}$

\begin{tabular}{rlr}
1 & COMPASS_DEV_ID & 592905 \\
0 & COMPASS_DEV_ID2 & 131874 \\
0 & COMPASS_DEV_ID3 & 263178 \\
6 & COMPASS_DIA_X & 1.041754 \\
0 & COMPASS_DIA_Y & 0.884046 \\
0 & COMPASS_DIA_Z & 1.029968 \\
0 & COMPASS_DIA2_X & 1.469129 \\
45 & COMPASS_DIA2_Y & 1.462009 \\
1 & COMPASS_DIA2_Z & 1.468962 \\
1 & COMPASS_DIA3_X & 1.051235 \\
4 & COMPASS_DIA3_Y & 1.015842 \\
1 & COMPASS_DIA3_Z & 1.089094 \\
0 & COMPASS_EXTERN2 & 0 \\
1 & COMPASS_EXTERN3 & 0 \\
7 & COMPASS_EXTERNAL & 1 \\
0 & COMPASS_FLTR_RNG & 0 \\
2 & COMPASS_LEARN & 0 \\
2 & COMPASS_MOT_X & 0 \\
0 & COMPASS_MOT_Y & 0 \\
3 & COMPASS_MOT_Z & 0 \\
0 & COMPASS_MOT2_X & 0 \\
0 & COMPASS_MOT2_Y & 0 \\
10 & COMPASS_MOT2_Z & 0 \\
-1 & COMPASS_MOT3_X & 0 \\
1 & COMPASS_MOT3_Y & -0.01018 \\
0 & COMPASS_MOT3_Z & 0.00556 \\
0 & COMPASS_MOTCT & 0.091549 \\
1 & COMPASS_ODI_X & 0.000654 \\
1100 & COMPASS_ODI_Y & 0.001744 \\
1300 & COMPASS_ODI_Z & 0.021579 \\
0 & COMPASS_ODI2_X & 0.025756 \\
0 & COMPASS_ODI2_Y & -0.00162 \\
1 & COMPASS_ODI2_Z & \\
1 & COMPASS_ODI3_X & COMPASS_ODI3_Y \\
\hline
\end{tabular}




\begin{tabular}{|c|c|c|c|c|c|}
\hline ARSPD_PIN & 65 & CAN_P2_DRIVER & 0 & COMPASS_ODI3_Z & -0.01183 \\
\hline ARSPD_PRIMARY & 0 & CHUTE_CHAN & 0 & COMPASS_OFFS_MAX & 850 \\
\hline ARSPD_PSI_RANGE & 1 & CHUTE_ENABLED & 0 & COMPASS_OFS_X & -36.0632 \\
\hline ARSPD_RATIO & 1.656156 & COMPASS_AUTO_ROT & 2 & COMPASS_OFS_Y & -79.4705 \\
\hline ARSPD_SKIP_CAL & 0 & COMPASS_AUTODEC & 1 & COMPASS_OFS_Z & -88.6375 \\
\hline ARSPD_TUBE_ORDER & 2 & COMPASS_CAL_FIT & 16 & COMPASS_OFS2_X & 131.9048 \\
\hline ARSPD_TYPE & 1 & COMPASS_DEC & 0.058186 & COMPASS_OFS2_Y & -183.726 \\
\hline COMPASS_OFS2_Z & -140.673 & EK2_MAG_I_GATE & 300 & FLOW_ENABLE & 0 \\
\hline COMPASS_OFS3_X & 73.21418 & EK2_MAG_M_NSE & 0.05 & FLOW_FXSCALER & 0 \\
\hline COMPASS_OFS3_Y & -61.507 & EK2_MAG_MASK & 0 & FLOW_FYSCALER & 0 \\
\hline COMPASS_OFS3_Z & 54.63035 & EK2_MAGB_P_NSE & 0.0001 & FLOW_ORIENT_YAW & 0 \\
\hline COMPASS_ORIENT & 0 & EK2_MAGE_P_NSE & 0.001 & FLOW_POS_X & 0 \\
\hline COMPASS_ORIENT2 & 0 & EK2_MAX_FLOW & 2.5 & FLOW_POS_Y & 0 \\
\hline COMPASS_ORIENT3 & 0 & EK2_NOAID_M_NSE & 10 & FLOW_POS_Z & 0 \\
\hline COMPASS_PMOT_EN & 0 & EK2_OGN_HGT_MASK & 0 & FLTMODE_CH & 8 \\
\hline COMPASS_PRIMARY & 0 & EK2_POS_I_GATE & 500 & FLTMODE1 & 10 \\
\hline COMPASS_TYPEMASK & 32 & EK2_POSNE_M_NSE & 1 & FLTMODE2 & 11 \\
\hline COMPASS_USE & 1 & EK2_RNG_I_GATE & 500 & FLTMODE3 & 5 \\
\hline COMPASS_USE2 & 1 & EK2_RNG_M_NSE & 0.5 & FLTMODE4 & 2 \\
\hline COMPASS_USE3 & 0 & EK2_RNG_USE_HGT & -1 & FLTMODE5 & 12 \\
\hline CRASH_ACC_THRESH & 0 & EK2_RNG_USE_SPD & 2 & FLTMODE6 & 0 \\
\hline CRASH_DETECT & 0 & EK2_TAU_OUTPUT & 25 & FORMAT_VERSION & 13 \\
\hline DSPOILR_RUD_RATE & 100 & EK2_TERR_GRAD & 0.1 & FS_GCS_ENABL & 0 \\
\hline EK2_ABIAS_P_NSE & 0.005 & EK2_VEL_I_GATE & 500 & FS_LONG_ACTN & 0 \\
\hline EK2_ACC_P_NSE & 0.6 & EK2_VELD_M_NSE & 0.7 & FS_LONG_TIMEOUT & 5 \\
\hline EK2_ALT_M_NSE & 3 & EK2_VELNE_M_NSE & 0.5 & FS_SHORT_ACTN & 0 \\
\hline EK2_ALT_SOURCE & 0 & EK2_WIND_P_NSE & 0.1 & FS_SHORT_TIMEOUT & 1.5 \\
\hline EK2_BCN_DELAY & 50 & EK2_WIND_PSCALE & 0.5 & GCS_PID_MASK & 0 \\
\hline EK2_BCN_I_GTE & 500 & EK2_YAW_I_GATE & 300 & GLIDE_SLOPE_MIN & 15 \\
\hline EK2_BCN_M_NSE & 1 & EK2_YAW_M_NSE & 0.5 & GLIDE_SLOPE_THR & 5 \\
\hline EK2_CHECK_SCALE & 150 & EK3_ENABLE & 0 & GND_ABS_PRESS & 98445.43 \\
\hline EK2_EAS_I_GATE & 400 & FBWA_TDRAG_CHAN & 0 & GND_ABS_PRESS2 & 98416.86 \\
\hline EK2_EAS_M_NSE & 1.4 & FBWB_CLIMB_RATE & 2 & GND_ABS_PRESS3 & 0 \\
\hline EK2_ENABLE & 1 & FBWB_ELEV_REV & 0 & GND_ALT_OFFSET & 0 \\
\hline EK2_FLOW_DELAY & 10 & FENCE_ACTION & 0 & GND_EXT_BUS & -1 \\
\hline EK2_FLOW_I_GATE & 300 & FENCE_AUTOENABLE & 0 & GND_FLTR_RNG & 0 \\
\hline EK2_FLOW_M_NSE & 0.25 & FENCE_CHANNEL & 0 & GND_PRIMARY & 0 \\
\hline EK2_GBIAS_P_NSE & 0.0001 & FENCE_MAXALT & 1000 & GND_TEMP & 0 \\
\hline EK2_GLITCH_RAD & 25 & FENCE_MINALT & 0 & GPS_AUTO_CONFIG & 1 \\
\hline EK2_GPS_CHECK & 31 & FENCE_RET_RALLY & 0 & GPS_AUTO_SWITCH & 1 \\
\hline EK2_GPS_DELAY & 220 & FENCE_RETALT & 0 & GPS_BLEND_MASK & 5 \\
\hline EK2_GPS_TYPE & 0 & FENCE_TOTAL & 0 & GPS_BLEND_TC & 10 \\
\hline EK2_GSCL_P_NSE & 0.0005 & FLAP_1_PERCNT & 0 & GPS_DELAY_MS & 0 \\
\hline
\end{tabular}




\begin{tabular}{|c|c|c|}
\hline EK2_GYRO_P_NSE & 0.03 & FLAP_1_SPEED \\
\hline EK2_HGT_DELAY & 60 & FLAP_2_PERCNT \\
\hline EK2_HGT_I_GATE & 500 & FLAP_2_SPEED \\
\hline EK2_IMU_MASK & 7 & FLAP_IN_CHANNEL \\
\hline EK2_LOG_MASK & 1 & FLAP_SLEWRATE \\
\hline EK2_MAG_CAL & 0 & FLOW_ADDR \\
\hline GPS_NAVFILTER & 8 & INS_ACCOFFS_Y \\
\hline GPS_POS1_X & 0 & INS_ACCOFFS_Z \\
\hline GPS_POS1_Y & 0 & INS_ACCSCAL_X \\
\hline GPS_POS1_Z & 0 & INS_ACCSCAL_Y \\
\hline GPS_POS2_X & 0 & INS_ACCSCAL_Z \\
\hline GPS_POS2_Y & 0 & INS_ENABLE_MASK \\
\hline GPS_POS2_Z & 0 & INS_FAST_SAMPLE \\
\hline GPS_RATE_MS & 200 & INS_GYR_CAL \\
\hline GPS_RATE_MS2 & 200 & INS_GYR_ID \\
\hline GPS_RAW_DATA & 0 & INS_GYR2_ID \\
\hline GPS_SAVE_CFG & 2 & INS_GYR2OFFS_X \\
\hline GPS_SBAS_MODE & 2 & INS_GYR2OFFS_Y \\
\hline GPS_SBP_LOGMASK & -256 & INS_GYR2OFFS_Z \\
\hline GPS_TYPE & 1 & INS_GYR3_ID \\
\hline GPS_TYPE2 & 0 & INS_GYR3OFFS_X \\
\hline GRIP_ENABLE & 0 & INS_GYR3OFFS_Y \\
\hline GROUND_STEER_ALT & 0 & INS_GYR3OFFS_Z \\
\hline GROUND_STEER_DPS & 90 & INS_GYRO_FILTER \\
\hline HIL_ERR_LIMIT & 5 & INS_GYROFFS_X \\
\hline HIL_MODE & 0 & INS_GYROFFS_Y \\
\hline HIL_SERVOS & 0 & INS_GYROFFS_Z \\
\hline HOME_RESET_ALT & 0 & INS_LOG_BAT_CNT \\
\hline ICE_ENABLE & 0 & INS_LOG_BAT_LGCT \\
\hline INITIAL_MODE & 0 & INS_LOG_BAT_LGIN \\
\hline INS_ACC_BODYFIX & 2 & INS_LOG_BAT_MASK \\
\hline INS_ACC_ID & 1442082 & INS_LOG_BAT_OPT \\
\hline INS_ACC2_ID & 1114914 & INS_NOTCH_ENABLE \\
\hline INS_ACC2OFFS_X & 0.093755 & INS_POS1_X \\
\hline INS_ACC2OFFS_Y & -0.43707 & INS_POS1_Y \\
\hline INS_ACC2OFFS_Z & 0.72873 & INS_POS1_Z \\
\hline INS_ACC2SCAL_X & 1.001637 & INS_POS2_X \\
\hline INS_ACC2SCAL_Y & 1.006893 & INS_POS2_Y \\
\hline INS_ACC2SCAL_Z & 1.030987 & INS_POS2_Z \\
\hline INS_ACC3_ID & 1442826 & INS_POS3_X \\
\hline INS_ACC3OFFS_X & 0.113791 & INS_POS3_Y \\
\hline INS_ACC3OFFS_Y & -0.2384 & INS_POS3_Z \\
\hline INS_ACC3OFFS_Z & -0.19322 & INS_PRODUCT_ID \\
\hline
\end{tabular}

EK2_GYRO_P_NSE

EK2_HGT_DELAY

EK2_HGT_I_GATE

EK2_IMU_MASK

EK2 MAG CAL

GPS_NAVFILTER

GPS_POS1_X

GPS_POS1_Y

GPS_POS1_Z

GPS POS2 $X$

GPS_POS2_Y

GPS_POS2_Z

GPS_RATE_MS

GPS_RATE_MS2

GPS_RAW_DATA

GPS_SAVE_CFG

GPS_SBAS_MODE

GPS_SBP_LOGMASK

GPS_TYPE

GPS_TYPE2

GRIP_ENABLE

GROUND_STEER_ALT

HIL_ERR_LIMIT

HIL_MODE

HIL_SERVOS

HOME_RESET_ALT

ICE_ENABLE

INITIAL_MODE

INS_ACC_BODYFIX

INS_ACC_ID

INS_ACC2_ID

INS_ACC2OFFS $X$

INS_ACC2OFFS_Y

INS_ACC2OFFS_Z

INS ACC2SCAL $X$

INS_ACC2SCAL_Y

INS_ACC2SCAL_Z

INS_ACC3_ID

INS_ACC3OFFS_X

INS_ACC3OFFS_Z $\begin{aligned} 0.03 & \text { FLAP_1_SPEED } \\ 60 & \text { FLAP_2_PERCNT }\end{aligned}$

0 GPS_DELAY_MS2 0

0 GPS GNSS MODE 0

0 GPS_GNSS_MODE2 0

0 GPS_INJECT_TO 127

75 GPS_MIN_DGPS 100

0 GPS_MIN_ELEV -100

0.132254 INVERTEDFLT_CH 0

-0.20538 KFF RDDRMIX 0

0.996505 KFF_THR2PTCH 0

0.999894 LAND_ABORT_DEG 0

0.997272 LAND_ABORT_THR 0

127 LAND_DISARMDELAY 20

1 LAND_DS_ABORTALT 0

1 LAND_DS_AIL_SCL 1

2359586 LAND_DS_APP_EXT 50

2229282 LAND_DS_ARSP_MAX 15

-0.0571 LAND_DS_ARSP_MIN 10

0.000316 LAND_DS_D 0

-0.03228 LAND_DS_ELEV_PWM 1500

2360330 LAND_DS_I 0

-0.01169 LAND_DS_IMAX 0

0.004807 LAND_DS_L1 30

0.01519 LAND_DS_L1_I 0

20 LAND_DS_L1_TCON $\quad 0.4$

-0.0145 LAND_DS_P 0

0.007241 LAND_DS_SLEW_SPD 0.5

0.003042 LAND_DS_SLOPE_A 1

1024 LAND_DS_SLOPE_B 1

32 LAND_DS_V_DWN 2

20 LAND_DS_V_FWD 1

0 LAND_DS_YAW_LIM 10

0 LAND_FLAP_PERCNT 0

0 LAND_FLARE_ALT 3

0 LAND_FLARE_SEC 2

0 LAND_PF_ALT 10

0 LAND_PF_ARSPD 0

0 LAND_PF_SEC 6

0 LAND_PITCH_CD 0

0 LAND_SLOPE_RCALC 2

0 LAND_THEN_NEUTRL 0

0 LAND_THR_SLEW 0

0 LAND_TYPE 0

5 LEVEL_ROLL_LIMIT 5 


\begin{tabular}{|c|c|c|}
\hline INS_ACC3SCAL_X & 0.994113 & INS_STILL_THRESH \\
\hline INS_ACC3SCAL_Y & 0.999787 & INS_TRIM_OPTION \\
\hline INS_ACC3SCAL_Z & 0.997391 & INS_USE \\
\hline INS_ACCEL_FILTER & 20 & INS_USE2 \\
\hline INS_ACCOFFS_X & -0.10872 & INS_USE3 \\
\hline LOG_DISARMED & 1 & NTF_BUZZ_PIN \\
\hline LOG_FILE_BUFSIZE & 16 & NTF_DISPLAY_TYPE \\
\hline LOG_FILE_DSRMROT & 0 & NTF_LED_BRIGHT \\
\hline LOG_MAV_BUFSIZE & 8 & NTF_LED_OVERRIDE \\
\hline LOG_REPLAY & 0 & NTF_LED_TYPES \\
\hline MAG_ENABLE & 1 & NTF_OREO_THEME \\
\hline MANUAL_RCMASK & 0 & OVERRIDE_CHAN \\
\hline MIN_GNDSPD_CM & 0 & OVERRIDE_SAFETY \\
\hline MIS_OPTIONS & 0 & PTCH2SRV_D \\
\hline MIS_RESTART & 0 & PTCH2SRV_FF \\
\hline MIS_TOTAL & 9 & PTCH2SRV_I \\
\hline MIXING_GAIN & 0.5 & PTCH2SRV_IMAX \\
\hline MIXING_OFFSET & 0 & PTCH2SRV_P \\
\hline MNT_ANGMAX_PAN & 4500 & PTCH2SRV_RLL \\
\hline MNT_ANGMAX_ROL & 4500 & PTCH2SRV_RMAX_DN \\
\hline MNT_ANGMAX_TIL & 4500 & PTCH2SRV_RMAX_UP \\
\hline MNT_ANGMIN_PAN & -4500 & PTCH2SRV_TCONST \\
\hline MNT_ANGMIN_ROL & -4500 & Q_ENABLE \\
\hline MNT_ANGMIN_TIL & -4500 & RALLY_INCL_HOME \\
\hline MNT_DEFLT_MODE & 3 & RALLY_LIMIT_KM \\
\hline MNT_JSTICK_SPD & 0 & RALLY_TOTAL \\
\hline MNT_LEAD_PTCH & 0 & RC_OVERRIDE_TIME \\
\hline MNT_LEAD_RLL & 0 & RC1_DZ \\
\hline MNT_NEUTRAL_X & 0 & RC1_MAX \\
\hline MNT_NEUTRAL_Y & 0 & RC1_MIN \\
\hline MNT_NEUTRAL_Z & 0 & RC1_REVERSED \\
\hline MNT_RC_IN_PAN & 0 & RC1_TRIM \\
\hline MNT_RC_IN_ROLL & 0 & RC10_DZ \\
\hline MNT_RC_IN_TILT & 0 & RC10_MAX \\
\hline MNT_RETRACT_X & 0 & RC10_MIN \\
\hline MNT_RETRACT_Y & 0 & RC10_REVERSED \\
\hline MNT_RETRACT_Z & 0 & RC10_TRIM \\
\hline MNT_STAB_PAN & 0 & RC11_DZ \\
\hline MNT_STAB_ROLL & 0 & RC11_MAX \\
\hline MNT_STAB_TILT & 0 & RC11_MIN \\
\hline MNT_TYPE & 0 & RC11_REVERSED \\
\hline NAV_CONTROLLER & 1 & RC11_TRIM \\
\hline NAVL1_DAMPING & 0.75 & RC12_DZ \\
\hline
\end{tabular}

\begin{tabular}{|c|c|c|}
\hline 0.1 & LIM_PITCH_MAX & 3000 \\
\hline 1 & LIM_PITCH_MIN & -2500 \\
\hline 1 & LIM_ROLL_CD & 4500 \\
\hline 1 & LOG_BACKEND_TYPE & 1 \\
\hline 1 & LOG_BITMASK & 65535 \\
\hline 0 & RC13_DZ & 0 \\
\hline 0 & RC13_MAX & 1934 \\
\hline 3 & RC13_MIN & 1094 \\
\hline 0 & RC13_REVERSED & 0 \\
\hline 199 & RC13_TRIM & 874 \\
\hline 0 & RC14_DZ & 0 \\
\hline 0 & RC14_MAX & 1934 \\
\hline 1 & RC14_MIN & 1094 \\
\hline 0.075 & RC14_REVERSED & 0 \\
\hline 0 & RC14_TRIM & 874 \\
\hline 0.153576 & RC15_DZ & 0 \\
\hline 1000 & RC15_MAX & 1900 \\
\hline 1.6 & RC15_MIN & 1100 \\
\hline 1 & RC15_REVERSED & 0 \\
\hline 75 & RC15_TRIM & 874 \\
\hline 75 & RC16_DZ & 0 \\
\hline 0.45 & RC16_MAX & 1900 \\
\hline 0 & RC16_MIN & 1100 \\
\hline 0 & RC16_REVERSED & 0 \\
\hline 5 & RC16_TRIM & 874 \\
\hline 0 & RC2_DZ & 30 \\
\hline 3 & RC2_MAX & 1939 \\
\hline 30 & RC2_MIN & 1099 \\
\hline 1939 & RC2_REVERSED & 0 \\
\hline 1099 & RC2_TRIM & 1518 \\
\hline 0 & RC3_DZ & 30 \\
\hline 1518 & RC3_MAX & 1939 \\
\hline 0 & RC3_MIN & 971 \\
\hline 1521 & RC3_REVERSED & 0 \\
\hline 1518 & RC3_TRIM & 1079 \\
\hline 0 & RC4_DZ & 30 \\
\hline 1518 & RC4_MAX & 1939 \\
\hline 0 & RC4_MIN & 1099 \\
\hline 2084 & RC4_REVERSED & 0 \\
\hline 951 & RC4_TRIM & 1518 \\
\hline 0 & RC5_DZ & 0 \\
\hline 1790 & RC5_MAX & 1835 \\
\hline 0 & RC5_MIN & 1203 \\
\hline
\end{tabular}




\begin{tabular}{|c|c|c|}
\hline NAVL1_LIM_BANK & 0 & RC12_MAX \\
\hline NAVL1_PERIOD & 17 & RC12_MIN \\
\hline NAVL1_XTRACK_I & 0.02 & RC12_REVERSED \\
\hline NTF_BUZZ_ENABLE & 1 & RC12_TRIM \\
\hline RC6_MIN & 1096 & RNGFND_PIN \\
\hline RC6_REVERSED & 0 & RNGFND_POS_X \\
\hline RC6_TRIM & 1939 & RNGFND_POS_Y \\
\hline RC7_DZ & 0 & RNGFND_POS_Z \\
\hline RC7_MAX & 2084 & RNGFND_PWRRNG \\
\hline RC7_MIN & 951 & RNGFND_RMETRIC \\
\hline RC7_REVERSED & 0 & RNGFND_SCALING \\
\hline RC7_TRIM & 1790 & RNGFND_SETTLE \\
\hline RC8_DZ & 0 & RNGFND_STOP_PIN \\
\hline RC8_MAX & 2084 & RNGFND_TYPE \\
\hline RC8_MIN & 951 & RNGFND2_ADDR \\
\hline RC8_REVERSED & 0 & RNGFND2_FUNCTION \\
\hline RC8_TRIM & 1790 & RNGFND2_GNDCLEAR \\
\hline RC9_DZ & 0 & RNGFND2_MAX_CM \\
\hline RC9_MAX & 1521 & RNGFND2_MIN_CM \\
\hline RC9_MIN & 1518 & RNGFND2_OFFSET \\
\hline RC9_REVERSED & 0 & RNGFND2_ORIENT \\
\hline RC9_TRIM & 1518 & RNGFND2_PIN \\
\hline RCMAP_PITCH & 2 & RNGFND2_POS_X \\
\hline RCMAP_ROLL & 1 & RNGFND2_POS_Y \\
\hline RCMAP_THROTTLE & 3 & RNGFND2_POS_Z \\
\hline RCMAP_YAW & 4 & RNGFND2_RMETRIC \\
\hline RELAY_DEFAULT & 0 & RNGFND2_SCALING \\
\hline RELAY_PIN & 54 & RNGFND2_SETTLE \\
\hline RELAY_PIN2 & 55 & RNGFND2_STOP_PIN \\
\hline RELAY_PIN3 & -1 & RNGFND2_TYPE \\
\hline RELAY_PIN4 & -1 & RPM_MAX \\
\hline RLL2SRV_D & 0.1 & RPM_MIN \\
\hline RLL2SRV_FF & 0 & RPM_MIN_QUAL \\
\hline RLL2SRV_I & 0.073626 & RPM_PIN \\
\hline RLL2SRV_IMAX & 500 & RPM_SCALING \\
\hline RLL2SRV_P & 0.7 & RPM_TYPE \\
\hline RLL2SRV_RMAX & 75 & RPM2_PIN \\
\hline RLL2SRV_TCONST & 0.45 & RPM2_SCALING \\
\hline RNGFND_ADDR & 0 & RPM2_TYPE \\
\hline RNGFND_FUNCTION & 0 & RSSI_TYPE \\
\hline RNGFND_GNDCLEAR & 10 & RST_MISSION_CH \\
\hline RNGFND_LANDING & 0 & RST_SWITCH_CH \\
\hline RNGFND_MAX_CM & 700 & RTL_AUTOLAND \\
\hline
\end{tabular}

NAVL1_LIM_BANK

NAVL1_PERIOD

NAVL1_XTRACK_I

RC6 MIN

RC6_REVERSED

RC6_TRIM

RC7_DZ

RC7_MAX

RC7_MIN

RC7_REVERSED

RC7_TRIM

RC8_DZ

RC8_MAX

RC8_MIN

RC8_REVERSED

RC8_TRIM

RC9_DZ

RC9_MAX

RC9_MIN

RC9_REVERSED

RC9_TRIM

RCMAP_PITCH

RCMAP ROLL

RCMAP_THROTTLE

RCMAP_YAW

RELAY_PIN

RELAY_PIN2

RELAY_PIN3

RELAY_PIN4

RLL2SRV_D

RLL2SRV_FF

RLL2SRV I

RLL2SRV_IMAX

RLL2SRV_P

RLL2SRV_RMAX

RLL2SRV_TCONST

RNGFND_ADDR

RNGFND_FUNCTION

RNGFND_LANDING

RNGFND_MAX_CM

\begin{tabular}{|c|c|c|}
\hline 2084 & RC5_REVERSED & 0 \\
\hline 951 & RC5_TRIM & 1517 \\
\hline 0 & RC6_DZ & 0 \\
\hline 1790 & RC6_MAX & 1939 \\
\hline-1 & SCALING_SPEED & 25 \\
\hline 0 & SCHED_DEBUG & 0 \\
\hline 0 & SCHED_LOOP_RATE & 50 \\
\hline 0 & SERIALO_BAUD & 115 \\
\hline 0 & SERIALO_PROTOCOL & 2 \\
\hline 1 & SERIAL1_BAUD & 57 \\
\hline 3 & SERIAL1_PROTOCOL & 1 \\
\hline 0 & SERIAL2_BAUD & 57 \\
\hline-1 & SERIAL2_PROTOCOL & 1 \\
\hline 0 & SERIAL3_BAUD & 38 \\
\hline 0 & SERIAL3_PROTOCOL & 5 \\
\hline 0 & SERIAL4_BAUD & 38 \\
\hline 10 & SERIAL4_PROTOCOL & 5 \\
\hline 700 & SERIAL5_BAUD & 57 \\
\hline 20 & SERIAL5_PROTOCOL & -1 \\
\hline 0 & SERIAL6_BAUD & 57 \\
\hline 25 & SERIAL6_PROTOCOL & -1 \\
\hline-1 & SERVO_AUTO_TRIM & 0 \\
\hline 0 & SERVO_RATE & 50 \\
\hline 0 & SERVO_SBUS_RATE & 50 \\
\hline 0 & SERVO_VOLZ_MASK & 0 \\
\hline 1 & SERVO1_FUNCTION & 4 \\
\hline 3 & SERVO1_MAX & 1855 \\
\hline 0 & SERVO1_MIN & 1183 \\
\hline-1 & SERVO1_REVERSED & 0 \\
\hline 0 & SERVO1_TRIM & 1500 \\
\hline 100000 & SERVO10_FUNCTION & 0 \\
\hline 10 & SERVO10_MAX & 1939 \\
\hline 0.5 & SERV010_MIN & 1096 \\
\hline 54 & SERVO10_REVERSED & 0 \\
\hline 1 & SERV010_TRIM & 1939 \\
\hline 0 & SERVO11_FUNCTION & 0 \\
\hline-1 & SERVO11_MAX & 1939 \\
\hline 1 & SERV011_MIN & 1099 \\
\hline 0 & SERV011_REVERSED & 0 \\
\hline 0 & SERV011_TRIM & 1939 \\
\hline 0 & SERV012_FUNCTION & 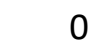 \\
\hline 0 & SERV012_MAX & 1939 \\
\hline 0 & SERVO12_MIN & 109 \\
\hline
\end{tabular}




$\begin{array}{lrl}\text { RNGFND_MIN_CM } & 20 & \text { RTL_RADIUS } \\ \text { RNGFND_OFFSET } & 0 & \text { RUDD_DT_GAIN } \\ \text { RNGFND_ORIENT } & 25 & \text { RUDDER_ONLY } \\ \text { SERVO13_MAX } & 1934 & \text { SERVO6_REVERSED } \\ \text { SERVO13_MIN } & 1094 & \text { SERVO6_TRIM } \\ \text { SERVO13_REVERSED } & 0 & \text { SERVO7_FUNCTION } \\ \text { SERVO13_TRIM } & 874 & \text { SERVO7_MAX } \\ \text { SERVO14_FUNCTION } & 0 & \text { SERVO7_MIN } \\ \text { SERVO14_MAX } & 1934 & \text { SERVO7_REVERSED } \\ \text { SERVO14_MIN } & 1094 & \text { SERVO7_TRIM } \\ \text { SERVO14_REVERSED } & 0 & \text { SERVO8_FUNCTION } \\ \text { SERVO14_TRIM } & 874 & \text { SERVO8_MAX } \\ \text { SERVO15_FUNCTION } & 0 & \text { SERVO8_MIN } \\ \text { SERVO15_MAX } & 1900 & \text { SERVO8_REVERSED } \\ \text { SERVO15_MIN } & 1100 & \text { SERVO8_TRIM } \\ \text { SERVO15_REVERSED } & 0 & \text { SERVO9_FUNCTION } \\ \text { SERVO15_TRIM } & 1500 & \text { SERVO9_MAX } \\ \text { SERVO16_FUNCTION } & 0 & \text { SERVO9_MIN } \\ \text { SERVO16_MAX } & 1900 & \text { SERVO9_REVERSED } \\ \text { SERVO16_MIN } & 1100 & \text { SERVO9_TRIM } \\ \text { SERVO16_REVERSED } & 0 & \text { SOAR_ENABLE } \\ \text { SERVO16_TRIM } & 1500 & \text { SRO_ADSB } \\ \text { SERVO2_FUNCTION } & 19 & \text { SRO_EXT_STAT } \\ \text { SERVO2_MAX } & 1937 & \text { SRO_EXTRA1 } \\ \text { SERVO2_MIN } & 1139 & \text { SRO_EXTRA2 } \\ \text { SERVO2_REVERSED } & 0 & \text { SRO_EXTRA3 } \\ \text { SERVO2_TRIM } & 1500 & \text { SRO_PARAMS } \\ \text { SERVO3_FUNCTION } & 70 & \text { SRO_POSITION } \\ \text { SERVO3_MAX } & 1939 & \text { SRO_RAW_CTRL } \\ \text { SERVO3_MIN } & 1079 & \text { SRO_RAW_SENS } \\ \text { SERVO3_REVERSED } & 0 & \text { SRO_RC_CHAN } \\ \text { SERVO3_TRIM } & 1079 & \text { SR1_ADSB } \\ \text { SERVO4_FUNCTION } & 21 & \text { SR1_EXT_STAT } \\ \text { SERVO4_MAX } & 1939 & \text { SR1_EXTRA1 } \\ \text { SERVO4_MIN } & 1096 & \text { SR1_EXTRA2 } \\ \text { SERVO4_REVERSED } & 0 & \text { SR1_EXTRA3 } \\ \text { SERVO4_TRIM } & 1518 & \text { SR1_PARAMS } \\ \text { SERVO5_FUNCTION } & 0 & \text { SR1_POSITION } \\ \text { SERVO5_MAX } & 1855 & \text { SR1_RAW_CTRL } \\ \text { SERVO5_MIN } & 1183 & \text { SR1_RAW_SENS } \\ \text { SERVO5_REVERSED } & 0 & \text { SR1_RC_CHAN } \\ \text { SERVO5_TRIM } & 1518 & \text { SR2_ADSB } \\ \text { SERVO6_FUNCTION } & 0 & \text { SR2_EXT_STTAT } \\ & & \end{array}$

RNGFND_MIN_CM

RNGFND_OFFSET

RNGFND_ORIENT

SERVO13_MAX

SERVO13_MIN

SERVO13_REVERSED

SERVO13_TRIM

SERV014_FUNCTION

SERVO14_MAX

SERV014_MIN

SERVO14_REVERSED

SERVO14_TRIM

SERVO15_FUNCTION

SERVO15 MAX

SERVO15_MIN

SERVO15_REVERSED

SERVO15_TRIM

SERVO16_FUNCTION

SERVO16_MAX

SERVO16_MIN

SERVO16_REVERSED

SERVO16_TRIM

SERVO2_FUNCTION

SERVO2_MAX

SERVO2_MIN

SERVO2_REVERSED

SERVO2_TRIM

SERVO3_FUNCTION

SERVO3_MAX

SERVO3_MIN

SERVO3_REVERSED

SERVO3_TRIM

SERVO4_FUNCTION

SERVO4_MAX

SERVO4_MIN

SERVO4_REVERSED

SERVO4_TRIM

SERVO5_FUNCTION

SERVO5_MAX

SERVO5_MIN

SERVO5_REVERSED

SERVO6_FUNCTION

\begin{tabular}{|c|c|c|}
\hline 0 & SERV012_REVERSED & 0 \\
\hline 10 & SERV012_TRIM & 1939 \\
\hline 0 & SERVO13_FUNCTION & 0 \\
\hline 0 & SR2_EXTRA3 & 2 \\
\hline 1939 & SR2_PARAMS & 10 \\
\hline 0 & SR2_POSITION & 2 \\
\hline 1939 & SR2_RAW_CTRL & 1 \\
\hline 1099 & SR2_RAW_SENS & 2 \\
\hline 0 & SR2_RC_CHAN & 2 \\
\hline 1939 & SR3_ADSB & 5 \\
\hline 0 & SR3_EXT_STAT & 1 \\
\hline 1939 & SR3_EXTRA1 & 1 \\
\hline 1096 & SR3_EXTRA2 & 1 \\
\hline 0 & SR3_EXTRA3 & 1 \\
\hline 1939 & SR3_PARAMS & 10 \\
\hline 0 & SR3_POSITION & 1 \\
\hline 1939 & SR3_RAW_CTRL & 1 \\
\hline 1099 & SR3_RAW_SENS & 1 \\
\hline 0 & SR3_RC_CHAN & 1 \\
\hline 1939 & STAB_PITCH_DOWN & 2 \\
\hline 0 & STALL_PREVENTION & 0 \\
\hline 5 & STAT_BOOTCNT & 40 \\
\hline 2 & STAT_FLTTIME & 676 \\
\hline 4 & STAT_RESET & $1.14 \mathrm{E}+08$ \\
\hline 4 & STAT_RUNTIME & 31798 \\
\hline 2 & STEER2SRV_D & 0.005 \\
\hline 10 & STEER2SRV_DRTFCT & 10 \\
\hline 2 & STEER2SRV_DRTMIN & 4500 \\
\hline 1 & STEER2SRV_DRTSPD & 0 \\
\hline 2 & STEER2SRV_FF & 0 \\
\hline 2 & STEER2SRV_I & 0.2 \\
\hline 5 & STEER2SRV_IMAX & 1500 \\
\hline 2 & STEER2SRV_MINSPD & 1 \\
\hline 4 & STEER2SRV_P & 1.8 \\
\hline 4 & STEER2SRV_TCONST & 0.75 \\
\hline 2 & STICK_MIXING & 1 \\
\hline 10 & SYS_NUM_RESETS & 58 \\
\hline 2 & SYSID_ENFORCE & 0 \\
\hline 1 & SYSID_MYGCS & 255 \\
\hline 2 & SYSID_THISMAV & 1 \\
\hline 2 & TECS_APPR_SMAX & 0 \\
\hline 5 & TECS_CLMB_MAX & 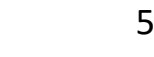 \\
\hline 2 & TECS_HGT_OMEGA & 3 \\
\hline
\end{tabular}




$\begin{array}{lrl}\text { SERVO6_MAX } & 1939 & \text { SR2_EXTRA1 } \\ \text { SERVO6_MIN } & 1099 & \text { SR2_EXTRA2 } \\ \text { TECS_LAND_DAMP } & 0.5 & \text { TECS_VERT_ACC } \\ \text { TECS_LAND_IGAIN } & 0 & \text { TELEM_DELAY } \\ \text { TECS_LAND_PDAMP } & 0 & \text { TERRAIN_ENABLE } \\ \text { TECS_LAND_PMAX } & 10 & \text { TERRAIN_FOLLOW } \\ \text { TECS_LAND_SINK } & 0.25 & \text { TERRAIN_LOOKAHD } \\ \text { TECS_LAND_SPDWGT } & -1 & \text { THR_FAILSAFE } \\ \text { TECS_LAND_SRC } & 0 & \text { THR_FS_VALUE } \\ \text { TECS_LAND_TCONST } & 2 & \text { THR_MAX } \\ \text { TECS_LAND_TDAMP } & 0 & \text { THR_MIN } \\ \text { TECS_LAND_THR } & -1 & \text { THR_PASS_STAB } \\ \text { TECS_PITCH_MAX } & 30 & \text { THR_SLEWRATE } \\ \text { TECS_PITCH_MIN } & -10 & \text { THR_SUPP_MAN } \\ \text { TECS_PTCH_DAMP } & 0 & \text { THROTTLE_NUDGE } \\ \text { TECS_RLL2THR } & 10 & \text { TKOFF_FLAP_PCNT } \\ \text { TECS_SINK_MAX } & 5 & \text { TKOFF_PLIM_SEC } \\ \text { TECS_SINK_MIN } & 2 & \text { TKOFF_ROTATE_SPD } \\ \text { TECS_SPD_OMEGA } & 2 & \text { TKOFF_TDRAG_ELEV } \\ \text { TECS_SPDWEIGHT } & 1 & \text { TKOFF_TDRAG_SPD1 } \\ \text { TECS_SYNAIRSPEED } & 0 & \text { TKOFF_THR_DELAY } \\ \text { TECS_THR_DAMP } & 0.5 & \text { TKOFF_THR_MAX } \\ \text { TECS_TIME_CONST } & 5 & \text { TKOFF_THR_MINACC } \\ \text { TECS_TKOFF_IGAIN } & 0 & \text { TKOFF_THR_MINSPD } \\ & & \end{array}$

$\begin{array}{rlr}4 & \text { TECS_INTEG_GAIN } & 0.1 \\ 4 & \text { TECS_LAND_ARSPD } & -1 \\ 7 & \text { TKOFF_THR_SLEW } & 0 \\ 0 & \text { TRIM_ARSPD_CM } & 3576 \\ 0 & \text { TRIM_AUTO } & 0 \\ 0 & \text { TRIM_PITCH_CD } & 0 \\ 2000 & \text { TRIM_THROTTLE } & 75 \\ 1 & \text { TUNE_CHAN } & 0 \\ 950 & \text { TUNE_CHAN_MAX } & 2000 \\ 100 & \text { TUNE_CHAN_MIN } & 1000 \\ 10 & \text { TUNE_ERR_THRESH } & 0.15 \\ 0 & \text { TUNE_MODE_REVERT } & 1 \\ 80 & \text { TUNE_PARAM } & 0 \\ 0 & \text { TUNE_RANGE } & 2 \\ 1 & \text { TUNE_SELECTOR } & 0 \\ 0 & \text { USE_REV_THRUST } & 2 \\ 2 & \text { WP_LOITER_RAD } & 182 \\ 0 & \text { WP_MAX_RADIUS } & 0 \\ 0 & \text { WP_RADIUS } & 91 \\ 0 & \text { YAW2SRV_DAMP } & 0.5 \\ 2 & \text { YAW2SRV_IMAX } & 500 \\ 0 & \text { YAW2SRV_INT } & 0 \\ 0 & \text { YAW2SRV_RLL } & 1 \\ 0 & \text { YAW2SRV_SLIP } & 0\end{array}$


VITA

Andrew Levi Ross

Candidate for the Degree of

Master of Science

\section{Thesis: DESIGN AND DEVELOPMENT OF A TORNADO INTERCEPT UNMANNED AERIAL VEHICLE}

\section{Major Field: Mechanical and Aerospace Engineering}

Biographical:

Education:

Completed the requirements for the Master of Science in Mechanical and Aerospace Engineering at Oklahoma State University, Stillwater, Oklahoma in December, 2019.

Completed the requirements for the Bachelor of Science in Mechanical and Aerospace Engineering at Oklahoma State University, Stillwater, Oklahoma in 2017.

\section{Experience:}

Graduate Teaching Assistant, Department of Mechanical and Aerospace Engineering, Oklahoma State University, August 2017-December 2019. Responsibilities include: assisting professors in grading all course material while both teaching and tutoring students.

Graduate Research Assistant, Department of Mechanical and Aerospace Engineering, Oklahoma State University, August 2017-December 2019. Research activities include: full design, fabrication, and integration of custom composite airframes; develop flight testing procedures for never-before-flown aircraft; flight testing and tuning of autopilots; diagnose turbojet errors; conduct routine airframe maintenance; pilot multi-rotor UAS for photogrammetric and geospatial applications while also conducting analysis on the data; among other activities.

Professional Memberships: American Institute of Aeronautics and Astronautics 